Dr. Martin Henk

Combinatorial Optimization

Konrad-Zuse-Zentrum für Informationstechnik Berlin

Phone +49/30/89604-284

Phone (Secretary) - 208

FAX $\quad+49 / 30 / 89604-125$

email henk@zib-berlin.de

\title{
Finite and Infinite Packings
}

\author{
Martin HenK
}

\section{Habilitationsschrift, Universität-GH-Siegen}

\author{
September 1994
}

Address after 01.01.1995:

Martin Henk

Technische Universität Berlin, Department of Mathematics, Sekr. MA 6-1

Straße des 17. Juni 136, D-10623 Berlin, Germany

Tel. $+49 / 30 / 31425735$

e-mail: henk@math.tu-berlin.de 


\section{Preface}

In this thesis we give a new approach to the classical problems of finite and infinite packings and lattice packings of convex bodies. This approach is based on the introduction of parameterized densities $\delta_{\rho}(K, C), \rho \in \mathbb{R}^{>0}$. The parameterized density of a finite packing set $C$ of a convex body $K$ with respect to the parameter $\rho$ is defined by

$$
\delta_{\rho}(K, C)=\frac{\#(C) V(K)}{V(\operatorname{conv}(C)+\rho K)},
$$

where $V(\cdot)$ denotes the volume and $\#(C)$ denotes the cardinality of the packing set $C$. In particular for $\rho=1$ we obtain a measure for the quality of finite packings, that is equivalent to the "densities" used by Rogers, Groemer, Oler and L. Fejes Tóth.

For a given convex body $K, \rho \in \mathbb{R}^{>0}$, and an integer $n$ we are asking for a packing set $C$ of cardinality $n$, that has a maximal parameterized density $\delta_{\rho}(K, C)$. Moreover we are interested in the asymptotic behavior of these maximal parameterized densities $\delta_{\rho}(K, n)$ with respect to $n$, i.e. we study $\delta_{\rho}(K)=$ $\lim \sup _{n \rightarrow \infty} \delta_{\rho}(K, n)$. The "density" $\delta_{\rho}(K)$ may be regarded as a "limit density" of finite packings of $K$ with respect to the parameter $\rho$.

For large values of $\rho$ it turns out that the limit density $\delta_{\rho}(K)$ is equal to $\delta(K)$, the density of a densest infinite packing. So the parameterized densities build a bridge between the finite and infinite packing problem. The least upper bound of the set of parameters $\rho$ with $\delta_{\rho}(K)=\delta(K)$ plays a special role in our investigation and is called critical parameter $\rho_{c}(K)$.

In order to characterize $\delta_{\rho}(K)$ for small parameters $\rho$, we transfer the notion of "sausage" packing sets, introduced by L. Fejes Tóth for finite packings of balls, to arbitrary convex bodies. A packing set $S_{n}(K)$ is called a densest sausage configuration of $K$ with cardinality $n$, if the volume of the convex hull of $S_{n}(K)+$ $K$ is minimal among all packing sets $C$ of cardinality $n$ and $\operatorname{dim}(C)=1$. The limit $\delta_{\rho}^{s}(K)=\lim _{n \rightarrow \infty} \delta_{\rho}\left(K, S_{n}(K)\right)$ may be considered as the parameterized density of a densest "infinite" sausage configuration of $K$. If $\rho$ is sufficiently small then $\delta_{\rho}(K)$ is equal to $\delta_{\rho}^{s}(K)$. In this case we are interested in the greatest lower bound of the set of parameters $\rho$ with $\delta_{\rho}(K)=\delta_{\rho}^{s}(K)$. This value of $\rho$ is called sausage parameter and is denoted by $\rho_{s}(K)$.

For the $d$-dimensional unit ball $B^{d}$ the determination of $\rho_{s}\left(B^{d}\right)$ is closely related to the "sausage conjecture" of L. Fejes Tóth. The conjecture says, that 
for $d \geq 5$ the density of a densest packing of $n$ balls with respect to the parameter $\rho=1$ is given by the density of a sausage configuration, i.e. $\delta_{1}\left(B^{d}, n\right)=$ $\delta_{1}\left(B^{d}, S_{n}\left(B^{d}\right)\right)$. A sausage parameter $\rho_{s}\left(B^{d}\right)>1$ implies that the sausage conjecture is true for sufficiently large $n$ in that dimension.

With respect to the functionals and problems mentioned above, the thesis is subdivided as follows:

Chapter 1 gives a short introduction in the theory of finite and infinite packings of convex bodies, where the last section deals in detail with the problem of finite packings of balls.

In chapter 2 (joint work with Ulrich Betke and Jörg M. Wills) we introduce the parameterized densities, the critical parameter and the sausage parameter. Furthermore, in the first section we state some basic properties of these densities and parameters, which also show the close connection to the density of a densest infinite packing. Section 2 deals with 2-dimensional packing problems. We give a description of the critical and sausage parameter by other functionals, which leads to best possible estimates of these parameters. In section 3 and 4 we give bounds for the critical and sausage parameter of arbitrary $d$-dimensional convex bodies.

Chapter 3 (joint work with Ulrich Betke and Jörg M. Wills) is devoted to "sausages". In the first part we consider finite packings of balls. We show that for every parameter $\rho<\sqrt{2}$ there exists a dimension $d_{0}(\rho)$ such that for $d \geq d_{0}(\rho)$ the maximal parameterized density $\delta_{\rho}\left(B^{d}, n\right)$ is attained by a sausage configuration. In the second part we prove a generalization of this result to centrally symmetric convex bodies.

In chapter 4 we show that the sausage conjecture of L. Fejes Tóth is true in dimensions $d \geq 45$.

In chapter 5 we investigate finite and infinite lattice packings. The necessary definitions are given in section 1, where by analogy with the general case, we introduce a critical lattice parameter and a sausage lattice parameter. Results of chapters 1 up to 4 concerning 2-dimensional packings, sausage configurations and sausage parameter can easily be adjusted to the "lattice case". In order to obtain an estimate for the critical lattice parameter, in section 2 we study a problem from the geometry of numbers related to lattice refinements.

At this place I want to thank Jörg M. Wills and Ulrich Betke for all the support and encouragement they have given me. The stimulating atmosphere that I have enjoyed at the "Lehrstuhl Wills" since finishing my studies in November 1988 til July 1994 has a great part in the setting of this work. In particular, I have to thank them for their permission to use results from joint papers here (cf. chapter 2 and 3$)$. 
Since August 1994 I am working in the research group of Günter M. Ziegler ${ }^{1}$ at the Konrad-Zuse-Zentrum in Berlin. I want to thank Günter M. Ziegler and the institute for their logistical and technical support.

I am grateful to Ulrich Betke, Lydia Tsintsifa and Günter M. Ziegler for many useful comments and discussions, and for reading and criticizing various versions of this thesis.

Berlin, September 1994.

Martin Henk

\footnotetext{
${ }^{1}$ Supported by a "Gerhard-Hess-Forschungsförderpreis 1994" of the German Science Foundation (DFG) (Zi 475/1-1).
} 


\section{Vorwort}

In der vorliegenden Arbeit beschreiben wir einen neuen Zugang zu den klassischen Problemen endlicher und unendlicher Packungen und Gitterpackungen konvexer Körper. Dieser neue Ansatz beruht auf der Einführung von parameterisierten Dichten $\delta_{\rho}(K, C), \rho \in \mathbb{R}^{>0}$. Die parameterisierte Dichte einer endlichen Packungsmenge $C$ eines konvexen Körpers $K$ bezüglich dem Parameter $\rho$ ist definiert durch

$$
\delta_{\rho}(K, C)=\frac{\#(C) V(K)}{V(\operatorname{conv}(C)+\rho K)} .
$$

Dabei bezeichnet $V(\cdot)$ das Volumen und \#(C) die Mächtigkeit der Menge $C$. Als Spezialfall erhält man für $\rho=1$ ein Maß für die Güte einer endlichen Packung, das zu den von Rogers, Groemer, Oler und L. Fejes Tóth verwendeten "Dichten" äquivalent ist.

Für einen gegebenen konvexen Körper $K, \rho \in \mathbb{R}^{>0}$, und eine natürliche Zahl $n$ fragen wir nach einer Packungsmenge $C$ mit Mächtigkeit $n$, welche die maximale parameterisierte Dichte $\delta_{\rho}(K, C)$ ergibt. Desweiteren untersuchen wir die asymptotische Entwicklung dieser maximalen parameterisierten Dichten $\delta_{\rho}(K, n)$ bzgl. $n$, d.h. wir betrachten die Größe $\delta_{\rho}(K)=\lim _{\sup _{n \rightarrow \infty}} \delta_{\rho}(K, n)$. Die "Dichte" $\delta_{\rho}(K)$ kann aufgefaßt werden als "Grenzdichte" endlicher Packungen von $K$ bzgl. dem Parameter $\rho$.

Es stellt sich nun heraus, daß für große Werte von $\rho$ die Grenzdichte $\delta_{\rho}(K)$ gleich $\delta(K)$, der Dichte einer dichtesten unendlichen Packung von $K$, ist. In diesem Sinne bilden die parameterisierten Dichten eine Brücke zwischen dem endlichen und unendlichen Packungsproblem. Die kleinste obere Schranke der Menge der Parameter $\rho$ mit der Eigenschaft $\delta_{\rho}(K)=\delta(K)$ ist von besonderem Interesse und heißt kritischer Parameter $\rho_{c}(K)$.

Um das Verhalten von $\delta_{\rho}(K)$ für kleine Werte von $\rho$ zu beschreiben, übertragen wir den von L. Fejes Tóth für endliche Kugelpackungen geprägten Begriff der "wurstförmigen" Packungsmengen auf beliebige konvexe Körper. Dabei verstehen wir unter einer dichtesten wurstförmigen Konfiguration von $K$ der Mächtigkeit $n$ eine Packungsmenge $S_{n}(K)$ mit der Eigenschaft, daß das Volumen der konvexen Hülle von $S_{n}(K)+K$ minimal ist, bzgl. aller Packungsmengen $C$ der Mächtigkeit $n$ mit $\operatorname{dim}(C)=1$. Der Grenzwert $\delta_{\rho}^{s}(K)=\lim _{n \rightarrow \infty} \delta_{\rho}\left(K, S_{n}(K)\right)$ kann als parameterisierte "Dichte" einer dichtesten "unendlichen" wurstförmigen Konfiguration von $K$ aufgefaßt werden. Ist $\rho$ hinreichend klein gewählt, so ergibt 
sich, daß $\delta_{\rho}(K)$ gleich $\delta_{\rho}^{s}(K)$ ist. In diesem Falle ist die größte untere Schranke der Menge der Parameter $\rho$ mit $\delta_{\rho}(K)=\delta_{\rho}^{s}(K)$ von besonderer Bedeutung und wird Wurst-Parameter $\rho_{s}(K)$ genannt.

Im Falle der $d$-dimensionalen Einheitskugel $B^{d}$ ist das Problem der Bestimmung von $\rho_{s}\left(B^{d}\right)$ eng mit der Wurstvermutung von L. Fejes Tóth verbunden, die behauptet, daß für $d \geq 5$ die dichteste endliche Packung von $n$ Kugeln, bzgl. der Dichte $\delta_{1}\left(B^{d}, C\right)$, von einer wurstförmigen Packungsmenge erzeugt wird, d.h. $\delta_{1}\left(B^{d}, n\right)=\delta_{1}\left(B^{d}, S_{n}\left(B^{d}\right)\right)$. Gilt in einer Dimension $\rho_{s}\left(B^{d}\right)>1$, so impliziert dies, daß die Wurstvermutung für hinreichend große $n$ in dieser Dimension richtig ist.

Bezüglich der hier beschriebenen Funktionale und Problematiken untergliedert sich die Arbeit wie folgt:

Kapitel 1 bietet eine kurze Einführung in die Theorie der endlichen und unendlichen Packungen konvexer Körper, dabei wird im letzten Abschnitt detailliert auf das Problem der endlichen Kugelpackungen eingegangen.

In Kapitel 2 (gemeinsame Arbeit mit Ulrich Betke und Jörg M. Wills) führen wir die parameterisierten Dichten ein, sowie den kritischen Parameter und WurstParameter. Zudem zeigen wir im ersten Abschnitt ein paar grundlegende Eigenschaften dieser Dichten und Parameter, die auch den engen Zusammenhang zu der Dichte einer dichtesten unendlichen Packung aufzeigen. Im zweiten Abschnitt behandeln wir 2-dimensionale Packungsprobleme. Insbesondere beschreiben wir dort den Wurst- und kritischen Parameter durch andere Funktionale und erhalten so bestmögliche Abschätzungen für diese Parameter. In Abschnitt 3 und 4 geben wir Schranken für den kritischen Parameter und Wurst-Parameter beliebiger $d$ dimensionaler konvexer Körper an.

Kapitel 3 (gemeinsame Arbeit mit Ulrich Betke und Jörg M. Wills) befaßt sich mit "Würsten". Im ersten Teil betrachten wir endliche Kugelpackungen. Wir zeigen, daß für jeden Parameterwert $\rho<\sqrt{2}$ eine Dimension $d_{0}(\rho)$ existiert, so daß für $d \geq d_{0}(\rho)$ die maximale parameterisierte Dichte $\delta_{\rho}\left(B^{d}, n\right)$ durch eine wurstförmige Konfiguration erzielt wird. Im zweiten Teil beweisen wir eine Verallgemeinerung dieses Resultats auf beliebige zentral-symmetrische konvexe Körper.

In Kapitel 4 zeigen wir, daß die Wurstvermutung von L. Fejes Tóth in Dimensionen $d \geq 45$ richtig ist.

In Kapitel 5 untersuchen wir endliche und unendliche Gitterpackungen. Die notwendigen Definitionen werden im ersten Abschnitt gegeben, und dort führen wir auch, analog zu dem allgemeinen Fall, einen Wurst-Gitterparameter und einen kritischen Gitterparameter ein. Resultate aus den Kapiteln 1 bis 4 bzgl. 2dimensionalen Packungen, wurstförmigen Konfigurationen und Wurst-Parameter lassen sich sofort auf den "Gitterfall" übertragen. Um eine Abschätzung für den kritischen Gitterparameter zu erhalten, untersuchen wir im zweiten Abschnitt ein Problem aus dem Bereich der Geometrie der Zahlen, welches sich mit Gitterverfeinerungen beschäftigt. 
An dieser Stelle möchte ich mich bei Herrn Prof. Dr. Jörg M. Wills und Herrn Prof. Dr. Ulrich Betke für die Unterstützung und die vielen Anregungen, die sie mir gegeben haben, bedanken. Die stimulierende Arbeitsatmosphäre am "Lehrstuhl Wills" in Siegen, die ich seit Beendigung meines Studiums im November 1988 bis zum Juli 1994 genossen habe, hat großen Anteil am Zustandekommen dieser Arbeit. Insbesondere danke ich ihnen für die Erlaubnis, Ergebnisse aus gemeinsamen Arbeiten für meine Habilitationsschrift zu verwenden (s. Kapitel 2 und 3).

Seit August 1994 arbeite ich in der Forschungsgruppe von Herrn Prof. Dr. Günter M. Ziegler, gefördert durch einen "Gerhard-Hess-Forschungsförderpreis 1994" der DFG (Zi 475/1-1), am Konrad-Zuse-Zentrum für Informationstechnik in Berlin. Ich danke Prof. Dr. Günter M. Ziegler und dem Institut für ihre logistische und technische Unterstützung.

Für die vielen nützlichen Kommentare und Diskussionen, und für das Lesen und Kritisieren verschiedener Versionen dieser Arbeit, danke ich Ulrich Betke, Lydia Tsintsifa und Günter M. Ziegler.

Berlin, September 1994

Martin Henk 


\section{Finite and Infinite Packings}

Preface/Vorwort i

1. Introduction — 1

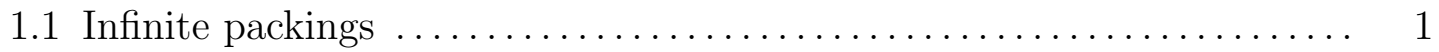

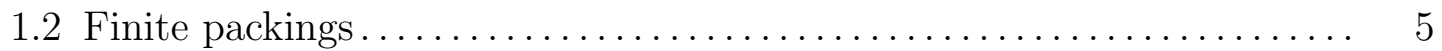

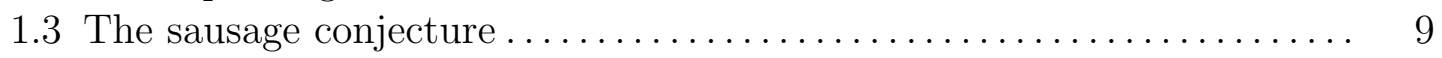

2. A new concept for finite and infinite packings $\quad 12$

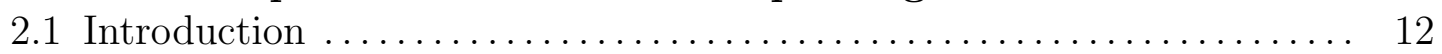

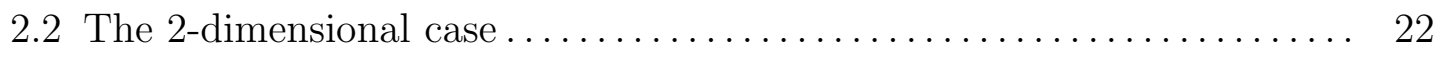

2.3 An upper bound for the critical parameter $\ldots \ldots \ldots \ldots \ldots \ldots \ldots \ldots . \ldots \ldots$

2.4 A lower bound for the sausage parameter $\ldots \ldots \ldots \ldots \ldots \ldots \ldots \ldots . \ldots \ldots$

3. Sausages are good packings 33

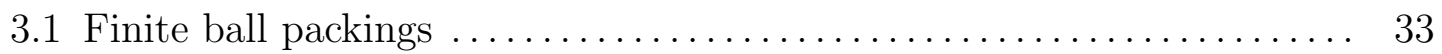

3.2 Finite packings of centrally symmetric bodies . . . . . . . . . . . 46

4. The Sausage conjecture 55

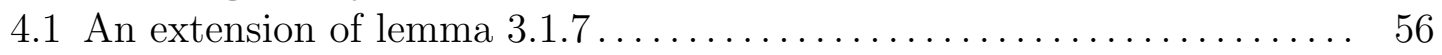

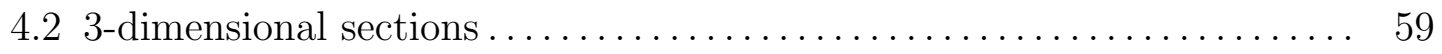

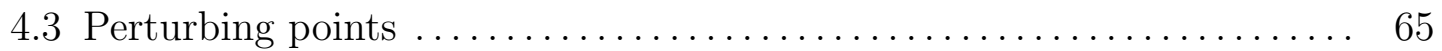

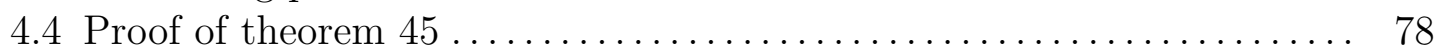

5. Lattice packings — 82

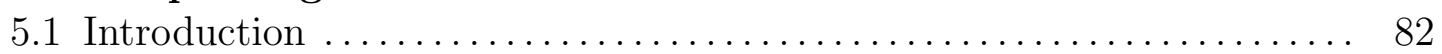

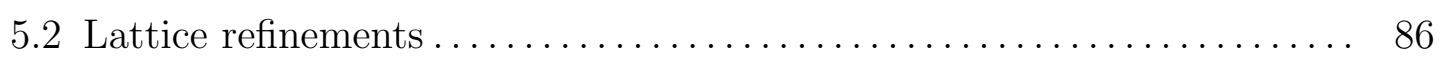

5.3 An upper bound for the critical lattice parameter ............... 91

$\begin{array}{ll}\text { Appendix } & 95\end{array}$

A.1 Partial derivatives of $f_{1,2}, f_{1,3}, f_{2,3}, g_{1,2}, g_{1,3}, g_{2,3} \ldots \ldots \ldots \ldots \ldots \ldots \ldots . \ldots 5$

$\begin{array}{ll}\text { References } & 107\end{array}$

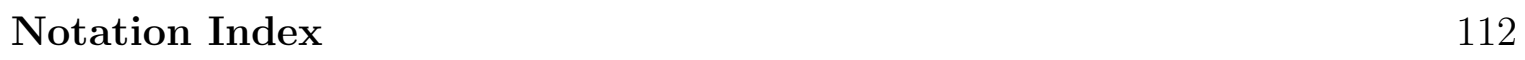




\section{Introduction}

In this thesis we study a new concept for finite and infinite packings of convex bodies. This concept was introduced by BeTKe, Henk \& Wills [BHW94a] and our aim is to demonstrate its power.

We only consider packings given by translates of a convex body, but instead of repeatedly talking about packings of translates we simply say packings. In this introduction we give a brief survey about classical results related to finite and infinite packings. For more information we refer to [CS93], [EGH89], [Fej83], [FK93], [Gru93], [GL87], [GW93], [Rog64].

\subsection{Infinite packings}

From an intuitive point of view the infinite packing problem for a convex body $K$ with non-empty interior in the $d$-dimensional Euclidean space $E^{d}$ is the following task:

Infinite packings. Find an arrangement of non-overlapping translates of $K$ such that the space $\left(E^{d}\right)$ is covered by this arrangement as good as possible.

In a mathematical sense this is not a well-defined problem, because we need a measure, that indicates how good (dense) the covering of the space by a given arrangement is. An obvious way to introduce such a measure is to approximate the space by a "large" cube and to consider the ratio of the volumes of the translates of $K$ lying in the cube to the volume of the cube. To make this more precise let the sum $S+T$ of two subsets $S, T \subset E^{d}$ be defined by $S+T=\{s+t$ : $s \in S, t \in T\}$ and for $\alpha \in \mathbb{R}$ let $\alpha S=\{\alpha s: s \in S\}$. Let $\mathcal{K}^{d}$ denote the set of all convex bodies — compact convex sets - with non-empty interior $(\operatorname{int}(K) \neq \emptyset)$ in $E^{d}$ and for $K \in \mathcal{K}^{d}$ define

$$
\mathcal{P}(K)=\left\{C \subset E^{d}:\left(x^{i}+\operatorname{int}(K)\right) \cap\left(x^{j}+\operatorname{int}(K)\right)=\emptyset, x^{i}, x^{j} \in C, x^{i} \neq x^{j}\right\} .
$$

$C \in \mathcal{P}(K)$ is called a packing set or (packing) configuration of $K$ and $C+K$ is called a packing or (packing) arrangement of $K$. Thus $\{C+K: C \in \mathcal{P}(K)\}$ is the set of all possible arrangements of non-overlapping translates of $K$. Further, for a subset $P \subset E^{d}$ let $V(P)$ denote the volume (Lebesgue measure) with respect to 
the affine hull of $P$ and for $\lambda \in \mathbb{R}^{>0}$ let $W_{\lambda}^{d}$ denote the $d$-dimensional cube with center 0 and edge length $2 \lambda$. With this notation we can define an appropriate measure as follows:

Definition 1.1.1 Let $K \in \mathcal{K}^{d}$ and $C \in \mathcal{P}(K)$.

$$
\delta(K, C)=\limsup _{\lambda \rightarrow \infty} \frac{1}{V\left(W_{\lambda}^{d}\right)} \sum_{x+K \subset W_{\lambda}^{d}, x \in C} V(K)
$$

is called the density of the packing set $C$ and

$$
\delta(K)=\sup \{\delta(K, C): C \in \mathcal{P}(K)\}
$$

is called the density of a densest packing of $K$.

The density of a packing set $C$ is invariant with respect to translations of $K$ or $C$, but it depends on the body chosen to approximate the space, i.e. if we replace in the definition the cube by another convex body we may obtain a different measure. On the other hand it turns out that $\delta(K)$ is independent of the "approximation body". This was pointed out by Groemer [Gro63], who moreover showed that there exists a $C \in \mathcal{P}(K)$ with

$$
\delta(K)=\delta(K, C)=\lim _{\lambda \rightarrow \infty} \frac{1}{V\left(W_{\lambda}^{d}\right)} \sum_{x+K \subset W_{\lambda}^{d}, x \in C} V(K) .
$$

Thus the notation of $\delta(K)$ as the density of a densest packing is justified and a packing $C+K$ with $\delta(K)=\delta(K, C)$ corresponds to the intuitive notion of a best possible arrangement.

Let us remark that there is also another approach to define the density of a densest packing: Let $K, L \in \mathcal{K}^{d}$ with $0 \in \operatorname{int}(L)$ and for $\lambda \in \mathbb{R}^{>0}$ let $n(K, \lambda L)$ be the maximal number of translates of $K$ that can be packed in $\lambda L$. Here "packed" means that two different translates have disjoint interiors. Then $\lim _{\lambda \rightarrow \infty} n(K, \lambda L)$ $V(K) / V(\lambda L)$ exists and does not depend on $L$. For the sphere this was shown by RANkin [Ran47] and in the general case by Hlawka [Hla49]. Groemer [Gro63] proved that this limit is equal to $\delta(K)$ and so we have

$$
\delta(K)=\lim _{\lambda \rightarrow \infty} \frac{n(K, \lambda L) V(K)}{V(\lambda L)} .
$$

Besides general infinite packings we are also interested in a more restricted type, lattice packings, which plays a central role in the theory of packings. For $K \in \mathcal{K}^{d}$ let

$$
\mathcal{P}^{*}(K)=\{\Lambda \in \mathcal{P}(K): \Lambda \text { is a lattice }\}
$$


be the set of all packing lattices in $\mathcal{P}(K)$. Here a packing lattice for $K \in \mathcal{K}^{d}$ is a discrete subgroup $\Lambda$ of $E^{d}$ generated by $d$ linearly independent vectors such that $\Lambda \in \mathcal{P}(K)$ (cf. [Gru93]). Now the infinite lattice packing problem is the task:

Infinite lattice packings. Find a lattice $\Lambda \in \mathcal{P}^{*}(K)$ such that the space $\left(E^{d}\right)$ is covered by $\Lambda+K$ as good as possible.

$\Lambda+K$ is called a lattice packing of $K$. Analogously to definition 1.1.1 we define:

Definition 1.1.2 Let $K \in \mathcal{K}^{d}$. Then

$$
\delta^{*}(K)=\sup \left\{\delta(K, \Lambda): \Lambda \in \mathcal{P}^{*}(K)\right\}
$$

is called the density of a densest lattice packing of $K$.

HLAWKA [Hla49] proved that $\lim _{\lambda \rightarrow \infty}\left(1 / V\left(W_{\lambda}^{d}\right)\right) \sum_{x+K \subset W_{\lambda}^{d}, x \in \Lambda} V(K)$ exists for all $K \in \mathcal{K}^{d}, \Lambda \in \mathcal{P}^{*}(K)$, and can be expressed as follows (see also [Min05], $[\operatorname{Rog} 64])$ :

$$
\delta(K, \Lambda)=\frac{V(K)}{\operatorname{det}(\Lambda)}
$$

where $\operatorname{det}(\Lambda)$ denotes the determinant of the lattice. Thus the determination of $\delta^{*}(K)$ is equivalent to the determination of $\inf \left\{\operatorname{det}(\Lambda): \Lambda \in \mathcal{P}^{*}(K)\right\}$. Now for a convex body this infimum is indeed a minimum (cf. [GL87]) and hence there exists a $\Lambda \in \mathcal{P}^{*}(K)$ with

$$
\delta^{*}(K)=\delta(K, \Lambda)=\frac{V(K)}{\operatorname{det}(\Lambda)}
$$

As in the general case the density of a densest lattice packing can also be defined via filling a convex body by another body. To this end let, similarly to (1.1.2), $n^{*}(K, \lambda L)$ be the maximal number of translates of $K$ that can be packed in $\lambda L$, $L \in \mathcal{K}^{d}, \lambda \in \mathbb{R}^{>0}$, such that the translates are part of a lattice packing. Then it was shown by HLAWKA [Hla49] that

$$
\delta^{*}(K)=\lim _{\lambda \rightarrow \infty} \frac{n^{*}(K, \lambda L) V(K)}{V(\lambda L)} .
$$

Obviously we have

$$
0<\delta^{*}(K) \leq \delta(K) \leq 1,
$$

and the upper bound is clearly best possible. Before we state a general lower bound for $\delta^{*}(K)$ (and thus for $\delta(K)$ ) we introduce the class $\mathcal{K}_{0}^{d} \subset \mathcal{K}^{d}$ of all centrally symmetric convex bodies. A body $K \in \mathcal{K}^{d}$ is called centrally symmetric 
if $x \in K$ implies $-x \in K$. For $K \in \mathcal{K}^{d}$ let $\mathrm{D} K=\frac{1}{2}(K-K)$ be the difference body of $K$. Then $\mathrm{D} K \in \mathcal{K}_{0}^{d}$ and it can easily be shown that

$$
C \in \mathcal{P}(K) \quad \Longleftrightarrow \quad C \in \mathcal{P}(\mathrm{D} K)
$$

This relation implies (cf. [Rog64], [Min05]):

$$
\delta(K)=\frac{V(K)}{V(\mathrm{D} K)} \delta(\mathrm{D} K), \quad \delta^{*}(K)=\frac{V(K)}{V(\mathrm{D} K)} \delta^{*}(\mathrm{D} K) .
$$

Hence the infinite packing problem for asymmetric convex bodies can be reduced to that for centrally symmetric convex bodies. Further, since

$$
2^{d} /\left(\begin{array}{c}
2 d \\
d
\end{array}\right) \leq \frac{V(K)}{V(\mathrm{D} K)} \leq 1
$$

every lower bound for $\delta^{*}(K)$ with respect to $K \in \mathcal{K}_{0}^{d}$ can be extended to a lower bound for $K \in \mathcal{K}^{d}$. The right hand side in (1.1.8) is an easy consequence of the Brunn-Minkowski theorem [BF34]; the left hand side is due to Rogers and SHEPHARD [RS57].

The best known lower bound for $\delta^{*}(K)$ is based on the famous MinkowskiHLAWKa theorem [Hla44], which for $K \in \mathcal{K}_{0}^{d}$ gives

$$
\delta^{*}(K) \geq \zeta(d) / 2^{d-1} \quad \text { with } \zeta(d)=\sum_{i=1}^{\infty} i^{-d}
$$

There are many refinements of this theorem, the sharpest one due to SCHMIDT [Sch63], who established the inequality

$$
\delta^{*}(K) \geq(c \cdot d) / 2^{d}
$$

where $c$ is a suitable absolute constant.

In the plane $(d=2)$ much more is known than for general dimensions. For $K \in \mathcal{K}_{0}^{2}$ we have

$$
\delta^{*}(K)=\delta(K)=V(K) / V(H(K)),
$$

where $H(K)$ is an affinely regular hexagon (possibly degenerate) of minimum area circumscribed to $K$. This result is a combination of works by L. FEJES TóTH [Fej50], Dowker [Dow44] and Reinhardt [Rei34]. On account of (1.1.7) this identity can be generalized to arbitrary convex bodies in $E^{2}$ :

$$
\delta^{*}(K)=\delta(K)=V(K) / V(H(\mathrm{D} K))
$$

Let us remark that Rogens $[\operatorname{Rog} 51]$ proved $\delta^{*}(K)=\delta(K)$ for $K \in \mathcal{K}^{2}$ independently of (1.1.10). 
The most investigated body with respect to $\delta^{*}(K)$ or $\delta(K)$ is, of course, the $d$-dimensional Euclidean unit ball $B^{d}$. This is a very natural problem, but also motivated by the relationship between $\delta^{*}\left(B^{d}\right)$ and the minimization of positive quadratic forms. Up to dimension 8 the values of $\delta^{*}\left(B^{d}\right)$ are known, but the determination of $\delta\left(B^{d}\right)$ is still open for $d \geq 3$. For $d=3$ this is the famous Kepler problem, see Muder [Mud93], Hsiang [Hsi93], Hales [Hal94] and HsiAng [Hsi94] for very recent progress and controversy. For a detailed survey about packings of balls we refer to the book [EGH89]. In the following we list some classical upper bounds for $\delta\left(B^{d}\right)$ (and thus for $\delta^{*}\left(B^{d}\right)$ ).

Chronologically, the first upper bound for $\delta\left(B^{d}\right)$ is due to BLICHFELDT [Bli29]:

$$
\delta\left(B^{d}\right) \leq \frac{d+2}{2} 2^{-d / 2} .
$$

A slightly better bound was given by RoGERs [Rog64]:

$$
\delta\left(B^{d}\right) \leq \sigma_{d} \sim \frac{d}{e} 2^{-d / 2} \quad(d \rightarrow \infty),
$$

where $\sigma_{d}$ is the ratio of the volume of that part of a regular $d$-dimensional simplex of edge length 2 covered by $d+1$ unit balls, centered at the vertices of the simplex, to the volume of the regular simplex. SidEL'NIKOv [Sid73], [Sid74] gave a considerable improvement of BLICHFELDT's bound:

$$
\delta\left(B^{d}\right) \leq 2^{-0.5096 d+o(d)}, \quad(d \rightarrow \infty) .
$$

A further improvement was given by KABATJANSKII \& LEVENŠTEIN [KL78]:

$$
\delta\left(B^{d}\right) \leq 2^{-0.599 d+o(d)}, \quad(d \rightarrow \infty) .
$$

Up to now this is the best upper bound. The best lower estimate known for $\delta^{*}\left(B^{d}\right)$ is due to BALL [Bal92], who proved

$$
\delta^{*}\left(B^{d}\right) \geq 2(d-1) \zeta(d) 2^{-d} .
$$

Observe that this bound is still of the same order of magnitude as the bound derived from the Minkowski-HLAwkA theorem (1.1.9), and the gap between the best upper and lower bound is exponential.

\section{$1.2 \quad$ Finite packings}

In view of (1.1.2) a natural way to pose a finite packing problem for a convex body $K \in \mathcal{K}^{d}$ is as follows: For a body $L \in \mathcal{K}^{d}$ find the maximal number $n(K, L)$ of translates of $K$ which can be packed in $L$. 
If the body $L$ is "large" enough, i.e. the inradius is large compared to the circumradius of $K$, then the ratio of $n(K, L) V(K)$ to the volume of $L$ will be nearly $\delta(K)$ (cf. (1.1.2)). For results related to this kind of finite packings we refer to the articles of Groemer [Gro63], [Gro85], Hlawka [Hla49] and for a discussion of this problem to the survey of Gritzmann \& WiLls [GW93].

In this thesis we are dealing with another type of finite packings: For a fixed $n \in \mathbb{N}$ and a $K \in \mathcal{K}^{d}$ we are interested in a convex body $L$ of minimal volume into which $n$ translates of $K$ can be packed. Suppose that we have found such an $L \in \mathcal{K}^{d}$ and let $x^{1}+K, \ldots, x^{n}+K$ be the translates of $K$ packed in $L$. Then necessarily we have $L=\operatorname{conv}\left(\cup_{i=1}^{n}\left(x^{i}+K\right)\right)=\operatorname{conv}\left\{x^{1}, \ldots, x^{n}\right\}+K$, where $\operatorname{conv}(S)$ denotes the convex hull of a set $S$; instead of $\operatorname{conv}\left(\left\{x^{1}, \ldots, x^{n}\right\}\right)$ we write $\operatorname{conv}\left\{x^{1}, \ldots, x^{n}\right\}$ for short. Thus in our sense the finite packing problem for $K \in \mathcal{K}^{d}$ is the following task:

Finite packings. For $n \in \mathbb{N}$ find an arrangement of $n$ non-overlapping translates of $K$ such that the volume of the convex hull of the arrangement is minimal.

Let us remark that it is also interesting to ask for a minimal arrangement with respect to other functionals (e.g. diameter or surface area) than the volume (cf. e.g. [KPW84], [Gri84], [Bör92], [Bör94]).

Before we introduce appropriate densities for finite packings we need one more piece of notation. For $K \in \mathcal{K}^{d}$ and $n \in \mathbb{N}$ let

$$
\mathcal{P}(K, n)=\{C \in \mathcal{P}(K): \#(C)=n\},
$$

where $\#(C)$ denotes the cardinality of the set $C$. Then $C \in \mathcal{P}(K, n)$ is called a packing set or (packing) configuration of $K$ with cardinality $n$ and $C+K$ is called a packing or (packing) arrangement of $K$ with cardinality $n$. If the cardinality of a packing set or a packing is clear from the context we omit the specification of the cardinality. Clearly $\{C+K: C \in \mathcal{P}(K, n)\}$ is the set of all possible arrangements of $n$ non-overlapping translates of $K$.

Now in view of the definition of densities for infinite packings (cf. (1.1.1)) it is reasonable to define densities for finite packings in the following way (cf. [Fej72], [Gri84]):

Definition 1.2.1 Let $n \in \mathbb{N}, K \in \mathcal{K}^{d}$ and $C \in \mathcal{P}(K)$ with $\#(C)<\infty$.

$$
\delta_{1}(K, C)=\frac{1}{V(\operatorname{conv}(C)+K)} \sum_{x \in C} V(K)=\frac{\#(C) \cdot V(K)}{V(\operatorname{conv}(C)+K)}
$$

is called the density of the finite packing set $C$ and

$$
\delta_{1}(K, n)=\sup \left\{\delta_{1}(K, C): C \in \mathcal{P}(K, n)\right\}
$$

is called the density of a densest finite packing of $K$ with cardinality $n$. 
The meaning of the index " 1 " in the notation of $\delta_{1}(\cdot, \cdot)$ will be explained in the next chapter, where we show that this finite density is just a special type of a more general definition of densities.

As a consequence of the theorem of BOLZANO-WEIERSTRASS we may replace "sup" by "max" in the above definition and thus there exists for every $n \in \mathbb{N}$ a $C \in \mathcal{P}(K, n)$ with

$$
\delta_{1}(K, n)=\frac{n \cdot V(K)}{V(\operatorname{conv}(C)+K)} .
$$

Also in the case of finite packings one is interested in packings generated by a lattice structure. But for clearness' sake we ignore this special type here. We will return to this topic in chapter 5 .

By definitions 1.1.1 and 1.2.1 we have (see also theorem 2.1.1 v)):

$$
\delta(K) \leq \limsup _{n \rightarrow \infty} \delta_{1}(K, n) .
$$

But in general it does not hold $\delta(K)=\limsup _{n \rightarrow \infty} \delta_{1}(K, n)$. For example: for $d \geq 3$ let $K \in \mathcal{K}_{0}^{d}$ be the Cartesian product of a $(d-1)$-dimensional unit ball and a line segment $[-u, u]$ for a certain vector $u \in E^{d}$ of unit length. Now for $n \in \mathbb{N}$ let $S_{n}(u, K)=\left\{x^{1}, \ldots, x^{n}\right\}$ with $x^{i}=2(i-1) \cdot u, 1 \leq i \leq n$. Then $S_{n}(u, K) \in \mathcal{P}(K, n)$ and $\operatorname{conv}\left(S_{n}(u, K)\right)+K$ is a cylinder of radius 1 and length $2 n$. Hence

$$
V\left(\operatorname{conv}\left(S_{n}(u, K)\right)+K\right)=2 n \kappa_{d-1}=n \cdot V(K),
$$

where $\kappa_{j}$ denotes the $j$-dimensional volume of the $j$-dimensional unit ball. By definition 1.2.1 we have $\delta_{1}(K, n) \leq 1$ and thus $\delta_{1}(K, n)=\delta_{1}\left(K, S_{n}(u, K)\right)=1$. Hence $\lim \sup _{n \rightarrow \infty} \delta_{1}(K, n)=1$. On the other hand for $d \geq 3$ the cylinder $K$ can not be packed such that the whole space is covered and so $\delta(K)<1$ (cf. [Sch61]).

A lower bound for $\delta_{1}(K, n)$ was given by Gritzmann [Gri85]. Together with the trivial upper bound 1 he obtained for $K \in \mathcal{K}^{d}$ the estimates:

$$
\frac{1}{d}<\frac{n}{d(n-1)+1} \leq \delta_{1}(K, n) \leq 1, \quad d \geq 2 .
$$

Hence optimal finite packings can not be too thin.

To compute the density $\delta_{1}(K, C)$ for a packing set $C$ we have to compute the volume of $\operatorname{conv}(C)+K$. This volume can be written with the mixed volumes $V(\operatorname{conv}(C), i, K, d-i)$ as the following sum (cf. e.g. [BF34], [Sch93]):

$$
V(\operatorname{conv}(C)+K)=\sum_{i=0}^{d}\left(\begin{array}{l}
d \\
i
\end{array}\right) V(\operatorname{conv}(C), i, K, d-i) .
$$

In our investigations we will often use the mixed volumes and so we write for abbreviation $V_{i}(\operatorname{conv}(C), K)$ instead of $V(\operatorname{conv}(C), i, K, d-i)$. In particular we have $V_{d}(\operatorname{conv}(C), K)=V(\operatorname{conv}(C)), V_{0}(\operatorname{conv}(C), K)=V(K)$ and for $i \geq 1$ :

$$
V_{i}(\operatorname{conv}(C), K) \geq 0, \text { with } V_{i}(\operatorname{conv}(C), K)=0 \Leftrightarrow \operatorname{dim}(\operatorname{conv}(C))<i \text {. }
$$


Formula (1.2.4) shows that the "size of the boundary of $\operatorname{conv}(C)$ with respect to $K$ ", "measured" by the mixed volumes $V_{1}(\operatorname{conv}(C), K), \ldots, V_{d-1}(\operatorname{conv}(C), K)$, has an essential influence to the volume of $\operatorname{conv}(C)+K$. Furthermore, this formula indicates that for finite packings there is not such an elegant way to obtain results for $\delta_{1}(K, C)$ from results for $\delta_{1}(\mathrm{D} K, C)$ as in the infinite case (cf. (1.1.7)). In fact it seems there is no way at all.

For the 2-dimensional case there are many results for finite packings of convex bodies (cf. [Fej72]). Here we state only those which are strongly connected to "our" finite packing problem. 1951 Rogers [Rog51], [Rog60] gave for $K \in \mathcal{K}_{0}^{2}$, $C \in \mathcal{P}(K, n)$ the bound:

$$
V(\operatorname{conv}(C)+K) \geq V(K)+(n-1) \frac{V(K)}{\delta(K)}
$$

which yields

$$
\delta_{1}(K, n) \leq \delta(K) \frac{n}{\delta(K)+n-1} .
$$

Together with (1.2.2) this implies for $K \in \mathcal{K}_{0}^{2}$ :

$$
\limsup _{n \rightarrow \infty} \delta_{1}(K, n)=\delta(K)
$$

So for 2-dimensional centrally symmetric convex bodies in the plane the densest finite densities converge to the density of a densest infinite packing. Recently, BÖRÖCZKY, JR. (personal communication) showed that this also holds for arbitrary plane convex bodies.

Actually Rogers proved the following result for $K \in \mathcal{K}^{2}$ :

$$
V(\operatorname{conv}(C))+2 V_{1}(\operatorname{conv}(C), \mathrm{D} K) \geq(n-1) \frac{V(K)}{\delta(K)} .
$$

For $K \in \mathcal{K}_{0}^{2}$ this implies (1.2.6) by (1.2.4). A result of a similar type for $K \in \mathcal{K}_{0}^{2}$ was given by OLER [Ole61] who showed for $C \in \mathcal{P}(K, n)$ :

$$
V(\operatorname{conv}(C))+\frac{V(K)}{\delta(K)} \frac{F_{2 K}(\operatorname{conv}(C))}{2} \geq(n-1) \frac{V(K)}{\delta(K)},
$$

where $F_{2 K}(\operatorname{conv}(C))$ denotes the Minkowski perimeter of $\operatorname{conv}(C)$ with respect to $2 K$ ([Lei79]). In fact, (1.2.8) is an improvement of (1.2.6), since for $K \in \mathcal{K}_{0}^{2}$ we have (cf. [Ole61])

$$
\frac{V(K)}{\delta(K)} \frac{F_{2 K}(\operatorname{conv}(C))}{2} \leq 2 V_{1}(\operatorname{conv}(C), K) .
$$


There are no high dimensional analogs of (1.2.6) and (1.2.8). But as for infinite packings we have some more information for the unit ball. First, in 1960, Groemer [Gro60] proved (1.2.8) for the case $K=B^{2}$ :

$$
V(\operatorname{conv}(C))+\frac{V\left(B^{2}\right)}{\delta\left(B^{2}\right)} \frac{F(\operatorname{conv}(C))}{4} \geq(n-1) \frac{V\left(B^{2}\right)}{\delta\left(B^{2}\right)},
$$

where $F(\operatorname{conv}(C))=2 F_{2 B^{2}}(\operatorname{conv}(C))$ denotes the perimeter of $\operatorname{conv}(C)$. All further results about finite ball packings are closely related to L. FEJEs Tóth's celebrated sausage conjecture.

\subsection{The sausage conjecture}

Let $S^{d-1}$ be the $(d-1)$-dimensional unit sphere and for $u \in S^{d-1}$ let $S_{n}\left(u, B^{d}\right)=$ $\left\{x^{1}, \ldots, x^{n}\right\} \in \mathcal{P}\left(B^{d}, n\right)$ with $x^{i}=2(i-1) u, 1 \leq i \leq n$. So $\operatorname{conv}\left(S_{n}\left(u, B^{d}\right)\right)+B^{d}$ is a cylinder of radius 1 and length $2(n-1)$ with two additional half balls at the ends. Thus (see also (2.1.3)):

$$
V\left(\operatorname{conv}\left(S_{n}\left(u, B^{d}\right)\right)+B^{d}\right)=2(n-1) \kappa_{d-1}+\kappa_{d} .
$$

Since $V\left(\operatorname{conv}\left(S_{n}\left(u, B^{d}\right)\right)+B^{d}\right)$ does not depend on the direction $u$ we write $S_{n}\left(B^{d}\right)$ for short. In 1975, L. FEJEs Tóth [Fej75] baptized the convex hull of $S_{n}\left(B^{d}\right)+B^{d}$ a "sausage" - hence we call $S_{n}\left(B^{d}\right)$ a sausage configuration - and postulated his famous

"Sausage" Conjecture: For $d \geq 5, n \in \mathbb{N}$ and $C \in \mathcal{P}\left(B^{d}, n\right)$

$$
V\left(\operatorname{conv}(C)+B^{d}\right) \geq V\left(\operatorname{conv}\left(S_{n}\left(B^{d}\right)\right)+B^{d}\right)
$$

with equality if and only if $C$ is a sausage configuration.

The inequality above is equivalent to

$$
\delta_{1}\left(B^{d}, n\right)=\delta_{1}\left(B^{d}, S_{n}\left(B^{d}\right)\right)=\frac{n \kappa_{d}}{2(n-1) \kappa_{d-1}+\kappa_{d}} .
$$

In other words: The sausage conjecture claims that the best possible arrangement of $n$ non-overlapping $d$-dimensional unit balls, $d \geq 5$, is given by a sausage arrangement $S_{n}\left(B^{d}\right)+B^{d}$.

Why $d \geq 5$ ? By the definitions 1.1.1 and 1.2.1 it is easy to see that we can "construct" a sequence of packings sets $C_{n} \in \mathcal{P}\left(B^{d}, n\right)$ with $\lim \sup _{n \rightarrow \infty} \delta_{1}\left(B^{d}\right.$, $\left.C_{n}\right)=\delta\left(B^{d}\right)($ cf. Theorem 2.1.1 v)). Hence a necessary condition for the conjecture is $\lim \sup _{n \rightarrow \infty} \delta_{1}\left(B^{d}, S_{n}\left(B^{d}\right)\right)>\delta\left(B^{d}\right)$. Now by (1.3.3) we obtain $\lim \sup _{n \rightarrow \infty}$ $\delta_{1}\left(B^{d}, S_{n}\left(B^{d}\right)\right)=\kappa_{d} /\left(2 \kappa_{d-1}\right)$ and from Rogers' upper bound for $\delta\left(B^{d}\right)(1.1 .13)$ it follows that for $d \geq 5$ a large sausage gives a better density than the density of 


\begin{tabular}{cccc} 
& $\begin{array}{c}\text { Rogers' } \\
d\end{array}$ & $\begin{array}{c}\text { sausage density } \\
\text { Upper bound }\end{array}$ & $\begin{array}{c}\delta_{1}^{s}\left(B^{d}\right) \\
\text { optimal lattice density }^{*}\left(B^{d}\right)\end{array}$ \\
\hline 2 & 0.906899 & 0.785398 & 0.906899 \\
3 & 0.779635 & 0.666666 & 0.740480 \\
4 & 0.647817 & 0.589048 & 0.616850 \\
5 & 0.525706 & 0.533333 & 0.465257 \\
6 & 0.419233 & 0.490873 & 0.372947 \\
7 & 0.329863 & 0.457142 & 0.295297 \\
8 & 0.256788 & 0.429514 & 0.253699
\end{tabular}

Table 1.3.1.

a densest infinite ball packing. In table 1.3.1 we list Rogers's bounds borrowed from a table in [Lee67], the values $\delta_{1}^{s}\left(B^{d}\right)=\limsup _{n \rightarrow \infty} \delta_{1}\left(B^{d}, S_{n}\left(B^{d}\right)\right)$, and the known density of $\delta^{*}\left(B^{d}\right)$ for $d=2, \ldots, 8$ (cf. [EGH89]).

There are several results supporting the conjecture. In 1982 BetKe, GritzMANN \& WILLS [BGW82] proved

$$
\operatorname{dim}(\operatorname{conv}(C)) \leq \frac{7}{12}(d-1) \quad \Rightarrow \quad(1.3 .2),
$$

i.e., a sausage configuration is best possible among all sufficiently "flat" packing sets $C$. For low dimensions even a stronger result holds: BetKe \& Gritzmann [BG84] showed

$$
\operatorname{dim}(\operatorname{conv}(C)) \leq 9 \text { and } d \geq \operatorname{dim}(\operatorname{conv}(C))+1 \quad \Rightarrow \quad(1.3 .2) .
$$

Thus for $5 \leq d \leq 10$ a packing set $C$ violating the conjecture must be full dimensional.

Kleinschmidt, Pachner \& Wills [KPW84] verified (1.3.2) for the case that $C$ differs only little from a sausage configuration. A result of a more general nature is due to Gritzmann (cf. [Gri84], [Gri86]) who established for $C \in \mathcal{P}\left(B^{d}, n\right):$

$$
\left(2+\sqrt{2}+\frac{2}{\sqrt{d-1}}\right) V\left(\operatorname{conv}(C)+B^{d}\right) \geq V\left(\operatorname{conv}\left(S_{n}\left(B^{d}\right)\right)+B^{d}\right),
$$

which implies that the sausage conjecture is correct up to a constant factor, essentially $2+\sqrt{2}$. In 1989 G. Fejes Tóth, Gritzmann \& Wills showed that a counterexample to the conjecture must be "fat" measured by the relationship between inradius and circumradius. The gap between this result and (1.3.4) was partially filled by BöröczKY, JR. \& HENK (cf. [Bör92], [BH92]) who proved 
(1.3.2) for all packing sets which are not "too fat" and not "too flat" measured by certain $i$-dimensional inradii and circumradii.

A first result confirming (1.3.2) without any restriction on $C \in \mathcal{P}\left(B^{d}, n\right)$ is due to BetKe, Henk \& WiLls [BHW94a]:

$$
V\left(\operatorname{conv}(C)+B^{d}\right) \geq V\left(\operatorname{conv}\left(S_{n}\left(B^{d}\right)\right)+B^{d}\right) \text { for } d \geq 13,387 .
$$

Hence the sausage conjecture is true for all sufficiently large dimensions. In chapter 4 we give a significant improvement of the dimension for which the conjecture holds $(d \geq 45)$.

Finally, here are some remarks about the situation in the dimensions 2,3,4. In the plane a sausage arrangement is never best possible for $n \geq 3$, cf. WEGNER [Weg86] for a complete discussion. In dimensions three and four it seems to be likely that for small $n$ the sausage $S_{n}\left(B^{d}\right)$ is best possible and from a certain number of balls full dimensional packing sets become extremal. For example, BÖRÖCZKY, JR. [Bör93] proved that for $d=3, n=4$ a sausage configuration is best possible, whereas GANDINI \& WILLS [GW92] calculated that for $d=3, n \geq$ 56 , suitable "pieces" of the densest packing lattice yield better arrangements than $S_{n}\left(B^{d}\right)$. The same holds for $d=4$ and $n \geq 367,300$ as pointed out by GANDINI \& ZUCCO [GZ92]. This phenomenon in dimension 3 and 4 was first mentioned in [Wil85] and referred to as the sausage catastrophe. 


\section{A new concept for finite and infinite packings}

In this chapter we introduce a family of densities for finite packings which build a bridge between the finite and infinite packing problem. The necessary definitions and some basic but important properties will be given in the next section.

These densities were defined by BetKe, Henk \& Wills in [BHW94a] and section 2.1, 2.2 and 2.3 are part of that paper. Furthermore, section 2.4 is part of the joint paper [BHW94b].

\section{$2.1 \quad$ Introduction}

Definition 2.1.1 Let $n \in \mathbb{N}, K \in \mathcal{K}^{d}$ and $C \in \mathcal{P}(K)$ with $\#(C)<\infty$. For $\rho \in \mathbb{R}^{>0}$ let

$$
\begin{aligned}
\delta_{\rho}(K, C) & =\frac{\#(C) \cdot V(K)}{V(\operatorname{conv}(C)+\rho \cdot K)}, \\
\delta_{\rho}(K, n) & =\sup \left\{\delta_{\rho}(K, C): C \in \mathcal{P}(K, n)\right\}, \\
\delta_{\rho}(K) & =\limsup _{n \rightarrow \infty} \delta_{\rho}(K, n) .
\end{aligned}
$$

For each $\rho>0$, the quotient $\delta_{\rho}(K, C)$ gives a "density measure" for the finite packing set $C, C \in \mathcal{P}(K) . \delta_{\rho}(K, n)$ is the density of a densest finite packing of cardinality $n$ with respect to that measure and $\delta_{\rho}(K)$ may be regarded as the "density" of a densest finite packing as $n$ tends to infinity. Sometimes we will also refer to $\delta_{\rho}(K, C)$ as the density of the configuration $C$ with respect to $K$ and $\rho$. Observe that $\delta_{1}(K, C)$ and $\delta_{1}(K, n)(\rho=1)$ are the classical finite densities defined in definition 1.2.1. By the theorem of BolzANO-WEIERSTRAsS we may write "max" instead of "sup" in the definition of $\delta_{\rho}(K, n)$ and thus, for every $n \in \mathbb{N}$, there exists a $C \in \mathcal{P}(K, n)$ with

$$
\delta_{\rho}(K, n)=\frac{n \cdot V(K)}{V(\operatorname{conv}(C)+\rho K)} .
$$


The densities $\delta_{\rho}(K, C), \delta_{\rho}(K, n)$ and $\delta_{\rho}(K)$ are called parameterized densities with parameter $\rho$.

Obviously, $\delta_{\rho}(K, C)=\delta_{\rho}(K, z+C)$ for $z \in E^{d}$ and therefore, we consider $C$ and $z+C$, for $z \in E^{d}$, as the "same" packing set, i.e., we do not distinguish between a packing set and its translates. In this sense, $S_{n}\left(B^{d}\right)$ and $z+S_{n}\left(B^{d}\right)$, $z \in E^{d}$, are the same sausage configurations.

One meaning of the parameter $\rho$ can be seen from the STEINER polynomial, which expresses $V(\operatorname{conv}(C)+\rho K)$ as polynomial in $\rho$ with the mixed volumes $V_{i}(\operatorname{conv}(C), K)$ as coefficients (cf. (1.2.4), [Sch93]):

$$
\begin{aligned}
V(\operatorname{conv}(C)+\rho K) & =\sum_{i=0}^{d}\left(\begin{array}{l}
d \\
i
\end{array}\right) \rho^{d-i} V_{i}(\operatorname{conv}(C), K) \\
& =V(\operatorname{conv}(C))+\rho^{d} V(K) \\
& +d \rho^{d-1} V_{1}(\operatorname{conv}(C), K)+\cdots+d \rho V_{d-1}(\operatorname{conv}(C), K) .
\end{aligned}
$$

Hence $\rho$ is a weight which controls the influence of the boundary of $\operatorname{conv}(C)$ on $V(\operatorname{conv}(C)+\rho K)$. The following small example illustrates that fact. Let $C \in \mathcal{P}\left(B^{2}, 7\right)$ be the configuration of seven points in the plane sketched in figure 2.1.1, and let $S_{7}\left(B^{2}\right)$ be a corresponding sausage configuration. For $\rho=1$ we have

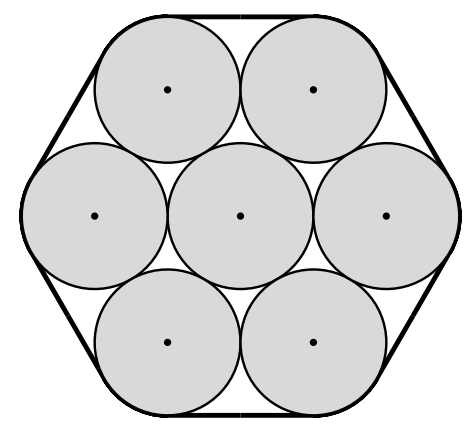

$$
\delta_{1}\left(B^{2}, C\right)=\frac{7 \pi}{6 \sqrt{3}+12+\pi} \sim 0.86
$$

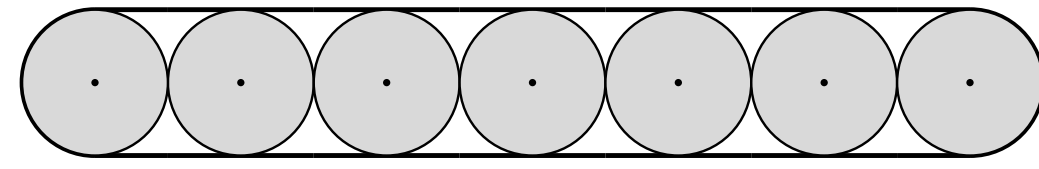

$$
\delta_{1}\left(B^{2}, S_{7}\left(B^{2}\right)\right)=\frac{7 \pi}{24+\pi} \sim 0.81
$$

Figure 2.1.1. 
So in this case the packing set $C$ yields a better density than $S_{7}\left(B^{2}\right)$. Since the surface area of $\operatorname{conv}\left(S_{7}\left(B^{2}\right)\right)(24)$ is relatively large compared with that of $\operatorname{conv}(C)(12)$ the superiority of $C$ will even increase when $\rho$ becomes larger. For $\rho=2$ we get

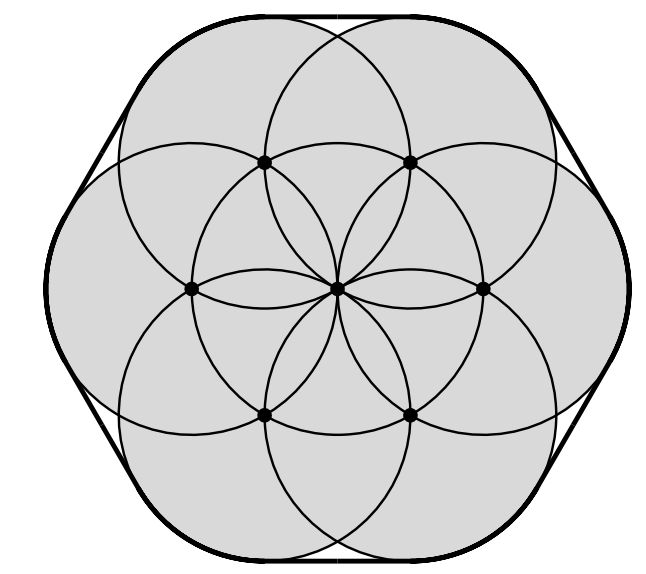

$$
\delta_{2}\left(B^{2}, C\right)=\frac{7 \pi}{6 \sqrt{3}+2 \cdot 12+2^{2} \cdot \pi} \sim 0.47
$$

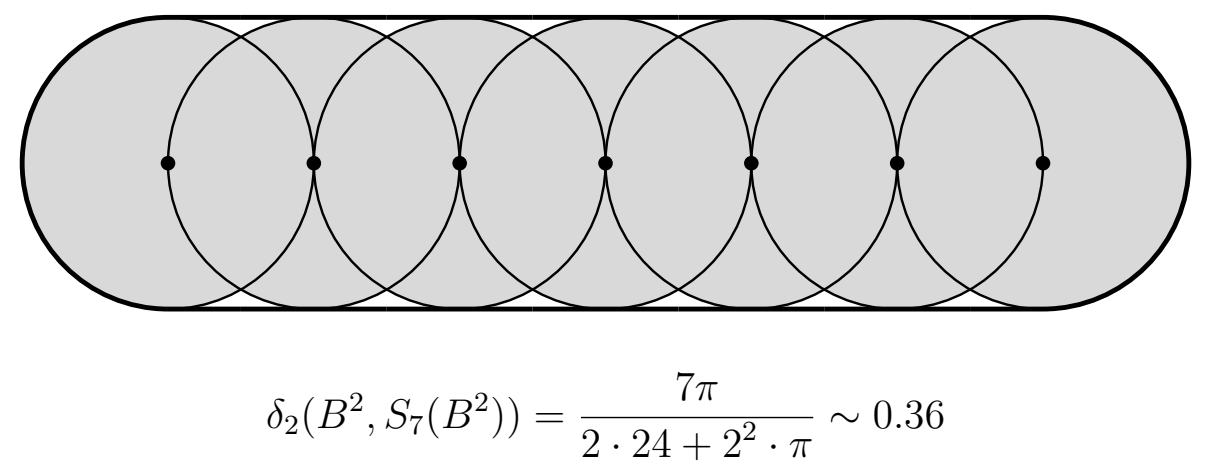

Figure 2.1.2.

On the other hand, a parameter $\rho<1$ lowers the influence of the boundary and for a sufficiently small $\rho$ the sausage configuration is better than $C$. Figure 2.1.3 shows this for $\rho=1 / 2$.

These pictures indicate that for $K \in \mathcal{K}^{d}$ and a large $\rho$, the parameterized density $\delta_{\rho}(K, n)$ attains its maximum for a packing set $C \in \mathcal{P}(K, n)$ with $\operatorname{dim}(C)=d$, whereas for small $\rho$ a 1-dimensional packing set becomes best possible. In fact, on account of (2.1.2) a large $\rho$ gives a strong weight to the mixed volumes with a small index $i$ and thus, in order to maximize $\delta_{\rho}(K, C), C \in \mathcal{P}(K, n)$, it seems to be 


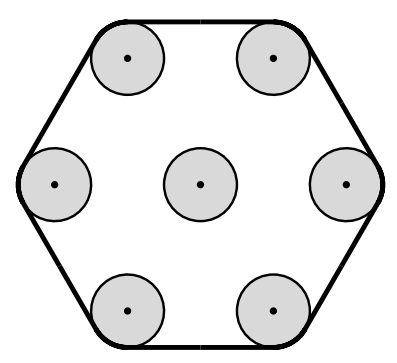

$$
\delta_{\frac{1}{2}}\left(B^{2}, C\right)=\frac{7 \pi}{6 \sqrt{3}+(1 / 2) \cdot 12+(1 / 2)^{2} \cdot \pi} \sim 1.28
$$

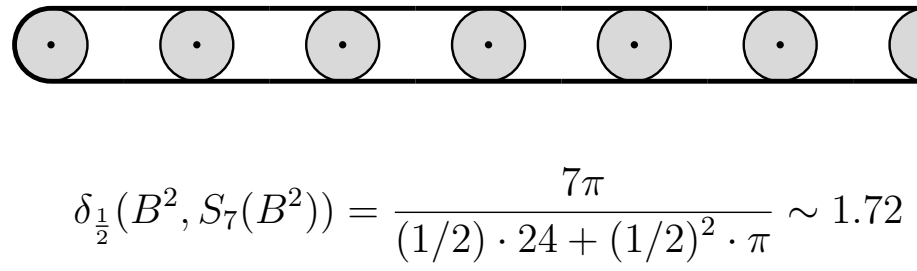

Figure 2.1.3.

reasonable to choose a full-dimensional $C$ such that $V_{i}(\operatorname{conv}(C), K) / V(\operatorname{conv}(C))$ is small. This implies that $\operatorname{conv}(C)$ should have a large inradius and therefore, we can expect that for a large $\rho$ the finite densest densities $\delta_{\rho}(K, n)$ converge to the classical density of a densest infinite packing of $K$ as $n$ tends to infinity. Hence we introduce:

Definition 2.1.2 For $K \in \mathcal{K}^{d}$ let

$$
\rho_{c}(K)=\inf \left\{\rho \in \mathbb{R}^{>0}: \delta_{\rho}(K)=\delta(K)\right\} .
$$

$\rho_{c}(K)$ is called the critical parameter of $K$.

It is not obvious that there exists a $\rho \in \mathbb{R}^{>0}$ with $\delta_{\rho}(K)=\delta(K)$, but with the usual convention inf $\emptyset=\infty$ the critical parameter is well-defined. In section 2.3 we will prove that $\rho_{c}(K)$ is bounded.

For small $\rho$ the mixed volumes with a large index $i$ are strongly weighted by $\rho$. In this case it seems to be better to choose a lower-dimensional packing set $C \in \mathcal{P}(K, n)$ than a full-dimensional one, with the extreme case of a 1-dimensional packing set (cf. (1.2.5)).

As we have seen in section 1.3 these 1-dimensional packing sets play a central role in the theory of finite ball packings and for $K=B^{d}$ the densest 1-dimensional packing sets are called sausage configurations. To introduce sausage configurations for arbitrary convex bodies we need the following notation. For $K \in \mathcal{K}_{0}^{d}$ let 
$f_{K}: E^{d} \rightarrow \mathbb{R}^{\geq 0}$ denote the distance function of $K$, i.e.,

$$
f_{K}(x)=\min \left\{\lambda \in \mathbb{R}^{\geq 0}: x \in \lambda K\right\}, \quad x \in E^{d} .
$$

According to (1.1.6) we have for each packing set $C \in \mathcal{P}(K), K \in \mathcal{K}^{d}$, that $f_{\mathrm{DK}}\left(x^{i}-x^{j}\right) \geq 2$ for $x^{i}, x^{j} \in C, x^{i} \neq x^{j}$. Hence the set of all packing sets of cardinality $n$ can also be described as

$$
\mathcal{P}(K, n)=\left\{\left\{x^{1}, \ldots, x^{n}\right\} \subset E^{d}: f_{\mathrm{D} K}\left(x^{i}-x^{j}\right) \geq 2,1 \leq i \neq j \leq n\right\} .
$$

Analogously to the definition of a sausage configuration for $B^{d}$ we define:

Definition 2.1.3 Let $K \in \mathcal{K}^{d}$ and $u \in S^{d-1}$.

$$
S_{n}(u, K)=\left\{2 \frac{i-1}{f_{\mathrm{D} K}(u)} u: 1 \leq i \leq n\right\}
$$

is called a sausage configuration of $K$ in direction $u$ with cardinality $n$ and $S_{n}(u, K)+K$ is called a sausage arrangement.

In other words, a sausage configuration of a body $K$ is a packing set $C \in \mathcal{P}(K)$ with $\operatorname{dim}(C)=1$ such that two adjacent translates touch each other. Figure 2.1.4 shows a sausage arrangement $S_{n}(u, K)+K$ of four equilateral triangles with the associated hexagon as difference body.

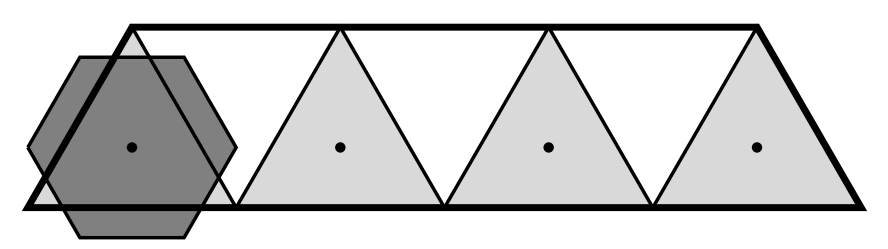

Figure 2.1.4.

To calculate the density of a sausage configuration with respect to a convex body $K$ and a parameter $\rho$ we denote by $P^{\perp}$ for a subset $P \subset E^{d}$ the orthogonal complement of the linear hull of $P$ and by $\left(K \mid P^{\perp}\right)$ we denote the orthogonal projection of $K$ onto $P^{\perp}$. Since $V_{1}\left(\operatorname{conv}\left(S_{n}(u, K)\right), K\right)=2(n-$ 1) $\left(f_{\mathrm{D} K}(u)\right)^{-1} V\left(K \mid u^{\perp}\right)$ (cf. [BF34]), we obtain on account of (2.1.2) and (1.2.5):

$$
V\left(\operatorname{conv}\left(S_{n}(u, K)\right)+\rho K\right)=2(n-1) \frac{V\left(K \mid u^{\perp}\right)}{f_{\mathrm{D} K}(u)} \rho^{d-1}+V(K) \rho^{d} .
$$


This is equivalent to

$$
\delta_{\rho}\left(K, S_{n}(u, K)\right)=\frac{n \cdot V(K)}{2(n-1)\left(f_{\mathrm{D} K}(u)\right)^{-1} V\left(K \mid u^{\perp}\right) \rho^{d-1}+V(K) \rho^{d}} .
$$

We see that in contrast to the unit ball the density of a sausage configuration of an arbitrary convex body depends on the chosen direction $u$. Since we are interested in maximal densities we set

$$
U(K)=\left\{v \in S^{d-1}: \frac{f_{\mathrm{D} K}(v)}{V\left(K \mid v^{\perp}\right)}=\max _{u \in S^{d-1}}\left\{\frac{f_{\mathrm{D} K}(u)}{V\left(K \mid u^{\perp}\right)}\right\}\right\} .
$$

By (2.1.4) we have for all $\rho \in \mathbb{R}^{>0}$ and $v \in U(K)$ :

$$
\delta_{\rho}\left(K, S_{n}(v, K)\right)=\max _{u \in S^{d-1}} \delta_{\rho}\left(K, S_{n}(u, K)\right) .
$$

For each $v \in U(K)$, the set $S_{n}(v, K)$ may be considered as a densest sausage configuration of $K$ with cardinality $n$. In the following let $S_{n}(K)$ be an arbitrary densest sausage configuration and let $u_{K} \in U(K)$ be the direction of $S_{n}(K)$, i.e. $S_{n}(K)=S_{n}\left(u_{K}, K\right)$.

The density of a densest sausage configuration of cardinality " $\infty$ " is of special interest in our investigation, and we define:

Definition 2.1.4 For $K \in \mathcal{K}^{d}$ and $\rho \in \mathbb{R}^{>0}$ let

$$
\delta_{\rho}^{s}(K)=\lim _{n \rightarrow \infty} \delta_{\rho}\left(K, S_{n}(K)\right)=\rho^{1-d} \cdot \frac{f_{\mathrm{D} K}\left(u_{K}\right) \cdot V(K)}{2 V\left(K \mid u_{K}^{\perp}\right)} .
$$

Finally, by analogy with the critical parameter we define with the usual convention $\sup (\emptyset)=-\infty$ :

Definition 2.1.5 For $K \in \mathcal{K}^{d}$ let

$$
\rho_{s}(K)=\sup \left\{\rho \in \mathbb{R}^{>0}: \delta_{\rho}(K)=\delta_{\rho}^{s}(K)\right\} .
$$

$\rho_{s}(K)$ is called the sausage parameter of $K$.

In section 2.4 we show that the sausage parameter is bounded from below. The sausage conjecture of L. FEJES Tóth states that $\delta_{1}\left(B^{d}, n\right)=\delta_{1}\left(B^{d}, S_{n}\left(B^{d}\right)\right)$ for $n \in \mathbb{N}$ and $d \geq 5$ (cf. (1.3.3)). By the definition above this would mean $\rho_{s}\left(B^{d}\right) \geq 1$ for $d \geq 5$. In chapter 4 we give, for high dimensions, an improvement of this lower bound.

Next we list some simple properties for the functionals introduced in the previous definitions. 
Theorem 2.1.1 Let $n \in \mathbb{N}, K \in \mathcal{K}^{d}$, and $C \in \mathcal{P}(K)$ with $\#(C)<\infty$.

i) $\delta_{\rho}(K, C), \delta_{\rho}(K, n), \delta_{\rho}(K)$ are monotonely decreasing in $\rho$.

ii) Let $A: E^{d} \rightarrow E^{d}$ be a non-singular affine transformation. Then $\delta_{\rho}(A K, A C)=\delta_{\rho}(K, C), \delta_{\rho}(A K, n)=\delta_{\rho}(K, n)$, and $\delta_{\rho}(A K)=\delta_{\rho}(K)$.

iii) $\min \left\{\frac{\rho^{1-d}}{d}, \rho^{-d}\right\}<\frac{n}{d(n-1) \rho^{d-1}+\rho^{d}} \leq \delta_{\rho}\left(K, S_{n}(K)\right)$

$$
\leq \delta_{\rho}(K, n) \leq \max \left\{\rho^{-d}, 1\right\} \text {. }
$$

iv) $\delta_{\rho}(K, C), \delta_{\rho}(K, n), \delta_{\rho}(K)$ are continuous in $\rho$.

v) There exist packing sets $C_{n} \in \mathcal{P}(K, n), n \in \mathbb{N}$, such that $\limsup _{n \rightarrow \infty} \delta_{\rho}\left(K, C_{n}\right)$ $=\delta(K)$ for all $\rho \in \mathbb{R}^{>0}$.

vi) $\delta_{\rho}(K) \geq \delta(K)$ for all $\rho \in \mathbb{R}^{>0}$.

\section{Proof.}

i) Since $V(\operatorname{conv}(C)+\rho K)$ is monotonely increasing in $\rho$ (cf. (2.1.2)), the quotient $\delta_{\rho}(K, C)$ is monotonely decreasing in $\rho$. Now let $\rho_{1}, \rho_{2} \in \mathbb{R}^{>0}$ with $\rho_{1}<\rho_{2}$ and let $C_{1}, C_{2} \in \mathcal{P}(K, n)$ such that $\delta_{\rho_{1}}(K, n)=\delta_{\rho_{1}}\left(K, C_{1}\right)$ and $\delta_{\rho_{2}}(K, n)=\delta_{\rho_{2}}\left(K, C_{2}\right)(\mathrm{cf} .(2.1 .1))$. Then

$$
\delta_{\rho_{1}}(K, n)=\delta_{\rho_{1}}\left(K, C_{1}\right) \geq \delta_{\rho_{1}}\left(K, C_{2}\right) \geq \delta_{\rho_{2}}\left(K, C_{2}\right)=\delta_{\rho_{2}}(K, n) .
$$

This also implies $\delta_{\rho_{1}}(K) \geq \delta_{\rho_{2}}(K)$.

ii) Let $A: E^{d} \rightarrow E^{d}$ be a non-singular affine map. It is easy to check that $C \in \mathcal{P}(K, n) \Leftrightarrow A C \in \mathcal{P}(A K, n)$ and $\delta_{\rho}(K, C)=\delta_{\rho}(A K, A C)$. Hence $\delta_{\rho}(K, n)=\delta_{\rho}(A K, n)$ and $\delta_{\rho}(K)=\delta_{\rho}(A K)$.

iii) By a result of Gritzmann [Gri85] we have $d V(K) \geq 2 V\left(K \mid u^{\perp}\right)\left(f_{\mathrm{D} K}(u)\right)^{-1}$ for $u \in S^{d-1}$ and together with (2.1.4)

$$
\delta_{\rho}(K, n) \geq \delta_{\rho}\left(K, S_{n}(K)\right) \geq \frac{n}{(n-1) d \rho^{d-1}+\rho^{d}} \geq \min \left\{\rho^{1-d} / d, \rho^{-d}\right\}
$$

If $\rho \leq 1$ then $\operatorname{conv}(C)+\rho K$ contains $n$ non-overlapping translates of $\rho K$ and thus $\delta_{\rho}(K, n) \leq \rho^{-d}$. Otherwise we know by i) $\delta_{\rho}(K, n) \leq \delta_{1}(K, n)$ and this shows the upper bound. 
iv) Obviously, $V(\operatorname{conv}(C)+\rho K)$ is continuous in $\rho$ and so is $\delta_{\rho}(K, C)$. Again, let $\rho_{1}, \rho_{2} \in \mathbb{R}^{>0}$ with $\rho_{1}<\rho_{2}$ and let $C_{1}, C_{2} \in \mathcal{P}(K, n)$ such that $\delta_{\rho_{1}}(K, n)=$ $\delta_{\rho_{1}}\left(K, C_{1}\right)$ and $\delta_{\rho_{2}}(K, n)=\delta_{\rho_{2}}\left(K, C_{2}\right)$. Then

$$
V\left(\operatorname{conv}\left(C_{2}\right)+\rho_{2} K\right) \leq V\left(\operatorname{conv}\left(C_{1}\right)+\rho_{2} K\right)
$$

and hence

$$
\begin{aligned}
& \delta_{\rho_{1}}(K, n)-\delta_{\rho_{2}}(K, n)=\frac{n V(K)}{V\left(\operatorname{conv}\left(C_{1}\right)+\rho_{1} K\right)}-\frac{n V(K)}{V\left(\operatorname{conv}\left(C_{2}\right)+\rho_{2} K\right)} \\
\leq & n V(K)\left(\frac{1}{V\left(\operatorname{conv}\left(C_{1}\right)+\rho_{1} K\right)}-\frac{1}{V\left(\operatorname{conv}\left(C_{1}\right)+\rho_{2} K\right)}\right) .
\end{aligned}
$$

In view of (2.1.2) this shows that $\delta_{\rho}(K, n)$ is LIPSCHITZ continuous. Clearly this implies that also $\delta_{\rho}(K)$ is continuous.

v) By (1.1.1) there exists an infinite packing set $C_{\infty}$ of $K$ with $\delta(K)=$ $\delta\left(K, C_{\infty}\right)$. We may assume that $C_{\infty}$ is saturated, i.e. $C_{\infty} \cup\{x\} \notin \mathcal{P}(K)$ for $x \in E^{d} \backslash C_{\infty}$. For $\lambda \in \mathbb{R}^{>0}$ let $C_{n_{\lambda}}$ be the maximal subset of $C_{\infty}$ with the property $C_{n_{\lambda}}+K \subset W_{\lambda}^{d}$ and let $\#\left(C_{n_{\lambda}}\right)=n_{\lambda}$. Thus $\delta(K)=$ $\lim _{\lambda \rightarrow \infty} n_{\lambda} V(K) / V\left(W_{\lambda}^{d}\right)$. Further let 0 be an interior point of $K$ and let $D(K)$ denote the diameter of $K$. With this notation we have $\operatorname{conv}\left(C_{n_{\lambda}}\right)+$ $\rho K \subset W_{\lambda}^{d}+\rho K \subset W_{\lambda}^{d}+\rho D(K) B^{d} \subset(1+\rho D(K) / \lambda) W_{\lambda}^{d}$ or

$$
\left(\frac{\lambda}{\lambda+\rho D(K)}\right)\left(\operatorname{conv}\left(C_{n_{\lambda}}\right)+\rho K\right) \subset W_{\lambda}^{d}
$$

which gives $\delta(K) \leq \lim \sup _{\lambda \rightarrow \infty} \delta_{\rho}\left(K, C_{n_{\lambda}}\right)$.

For the reverse direction let $\nu(\lambda)$ be the radius of a maximal $d$-ball contained in $\operatorname{conv}\left(C_{n_{\lambda}}\right)$ and centered at the origin. Since $C_{\infty}$ is saturated it follows $W_{\lambda-D(K)}^{d} \subset \operatorname{conv}\left(C_{n_{\lambda}}\right)+D(K) B^{d} \subset(1+D(K) / \nu(\lambda)) \operatorname{conv}\left(C_{n_{\lambda}}\right), \lambda>D(K)$. Hence

$$
W_{\lambda}^{d} \subset\left(\frac{\lambda}{\lambda-D(K)} \frac{\nu(\lambda)+D(K)}{\nu(\lambda)}\right)\left(\operatorname{conv}\left(C_{n_{\lambda}}\right)+\rho K\right)
$$

and so $\delta(K) \geq \lim \sup _{\lambda \rightarrow \infty} \delta_{\rho}\left(K, C_{n_{\lambda}}\right)$.

vi) Immediate consequence of $\mathrm{v}$ ).

Theorem 2.1.2 Let $K \in \mathcal{K}^{d}$ and $0<\rho_{s}(K), \rho_{c}(K)<\infty$.
i) $\delta_{\rho}(K)=\delta_{\rho}^{s}(K)$ for $\rho \in\left(0, \rho_{s}(K)\right]$.
ii) $\delta_{\rho}(K)=\delta(K)$ for $\rho \in\left[\rho_{c}(K), \infty\right)$.
iii) $\rho_{s}(K) \leq \rho_{c}(K)$. 
iv) Let $n \in \mathbb{N}$ and $\rho^{\prime}>0$ with $\delta_{\rho^{\prime}}(K, n)=\delta_{\rho^{\prime}}\left(K, S_{n}(K)\right)$. Then for $0<$ $\rho<\rho^{\prime}$ each $C \in \mathcal{P}(K, n)$ with $\delta_{\rho}(K, C)=\delta_{\rho}(K, n)$ is a densest sausage configuration.

Proof.

i) Since $0<\rho_{s}(K)<\infty$ we have by theorem 2.1.1 iv) that $\delta_{\rho_{s}(K)}(K)=$ $\delta_{\rho_{s}(K)}^{s}(K)$. Let $\rho_{1}<\rho_{s}(K)$. By definition 2.1.1 there exists a sequence $\left\{n_{j}\right\} \subset \mathbb{N}$ with $\lim _{j \rightarrow \infty} \delta_{\rho_{1}}\left(K, n_{j}\right)=\delta_{\rho_{1}}(K)$. With regard to definition 2.1.5 we may assume $\delta_{\rho_{s}(K)}\left(K, n_{j}\right) \leq \delta_{\rho_{s}(K)}\left(K, S_{n_{j}}(K)\right)+o(1)$ as $j \rightarrow \infty$. This is equivalent to (cf. (2.1.2), (2.1.4))

$2\left(n_{j}-1\right) \frac{V\left(K \mid u_{K}^{\perp}\right)}{f_{\mathrm{D} K}\left(u_{K}\right)}\left(\rho_{s}(K)\right)^{d-1} \leq \sum_{i=1}^{d}\left(\begin{array}{l}d \\ i\end{array}\right) V_{i}\left(\operatorname{conv}\left(C_{n_{j}}\right), K\right)\left(\rho_{s}(K)\right)^{d-i}+o(1)$,

for each packing set $C_{n_{j}} \in \mathcal{P}\left(K, n_{j}\right)$ and $j \rightarrow \infty$. The mixed volumes are non-negative and thus the inequality above holds for each $\rho \in\left(0, \rho_{s}(K)\right]$, which yields

$$
\delta_{\rho_{1}}\left(K, n_{j}\right) \leq \delta_{\rho_{1}}\left(K, S_{n_{j}}(K)\right)+o(1), \quad j \rightarrow \infty .
$$

On the other hand we have $\delta_{\rho_{1}}\left(K, n_{j}\right) \geq \delta_{\rho_{1}}\left(K, S_{n_{j}}(K)\right)$. Hence $\delta_{\rho_{1}}(K)=$ $\delta_{\rho_{1}}^{s}(K)$.

ii) Again, since $\rho_{c}(K)$ is bounded we have $\delta_{\rho_{c}(K)}(K)=\delta(K)$. Let $\rho_{2} \geq \rho_{c}(K)$. By theorem 2.1.1 i) and vi) we get $\delta(K) \leq \delta_{\rho_{2}}(K) \leq \delta_{\rho_{c}(K)}(K)=\delta(K)$, which shows $\delta_{\rho_{2}}(K)=\delta(K)$.

iii) Follows from i) and ii).

iv) Suppose there exists a $\rho_{0}<\rho^{\prime}$ and a $C \in \mathcal{P}(K, n)$ with $\delta_{\rho_{0}}(K, n)=$ $\delta_{\rho_{0}}(K, C)$ and $C$ is not a sausage configuration. Then we may assume $\operatorname{dim}(C) \geq 2$. By assumption we have (cf.(2.1.5))

$$
2(n-1) \frac{V\left(K \mid u_{K}^{\perp}\right)}{f_{\mathrm{D} K}\left(u_{K}\right)}\left(\rho^{\prime}\right)^{d-1} \leq \sum_{i=1}^{d}\left(\begin{array}{l}
d \\
i
\end{array}\right) V_{i}(\operatorname{conv}(C), K)\left(\rho^{\prime}\right)^{d-i} .
$$

and on account of $V_{2}(\operatorname{conv}(C), K)>0$ (cf. (1.2.5)) this inequality yields the contradiction $V\left(\operatorname{conv}\left(S_{n}(K)\right)+\rho_{0} K\right)<V\left(\operatorname{conv}(C)+\rho_{0} K\right)$.

So if we regard $\delta_{\rho}(K)$ for a fixed $K \in \mathcal{K}^{d}$ as a function in $\rho$ then we "know" the function in the intervals $\left(0, \rho_{s}(K)\right]$ and $\left[\rho_{c}(K), \infty\right)$.

Another simple but important property of $\rho_{s}(K)$ and $\rho_{c}(K)$ that shows the close relation between these functionals and the density of a densest infinite packing is given by: 
Theorem 2.1.3 Let $K \in \mathcal{K}^{d}$ and $0<\rho_{s}(K), \rho_{c}(K)<\infty$. Then

$$
\delta_{\rho_{c}(K)}^{s}(K) \leq \delta(K) \leq \delta_{\rho_{s}(K)}^{s}(K)
$$

Proof. By the definition of $\delta_{\rho}(K)$ and $\rho_{c}(K)$ we have $\delta_{\rho_{c}(K)}^{s}(K) \leq \delta_{\rho_{c}(K)}(K)=$ $\delta(K)$. Further by the definition of $\rho_{s}(K)$ and theorem 2.1.1 vi) we get $\delta(K) \leq$ $\delta_{\rho_{s}(K)}(K)=\delta_{\rho_{s}(K)}^{s}(K)$.

In the next section we will prove $\rho_{s}(K)=\rho_{c}(K)$ for $K \in \mathcal{K}^{2}$ and by the theorem above we obtain $\delta(K)=\delta_{\rho_{c}(K)}^{s}(K)=\delta_{\rho_{s}(K)}^{s}(K)$. But at least for $d \geq 4$ no similar result can be expected as the following theorem shows (cf. [Gri84]).

Theorem 2.1.4 For $d \geq 4$ there exists a $K \in \mathcal{K}_{0}^{d}$ with

$$
\rho_{s}(K)<1<\rho_{c}(K)
$$

Proof. Let $K \in \mathcal{K}_{0}^{d}$ be the Cartesian product of a regular hexagon $H$ and $B^{d-2}$. Since $H$ generates a tiling of its associated linear 2-dimensional plane we obtain $\delta_{1}(K)=1$. On the other hand as $d \geq 4, K$ does not tile $E^{d}$, and thus $\delta_{\rho_{c}(K)}(K)=\delta(K)<1$, which means $\rho_{c}(K)>1$ by theorem 2.1.2 ii). Furthermore, we have $\delta_{1}^{s}(K)<1=\delta_{1}(K)$ which shows $\rho_{s}(K)<1$ (cf. theorem 2.1.2 i)).

Nevertheless theorem 2.1.3 implies that any upper bound of $\rho_{c}(K)$ gives a lower bound for $\delta(K)$ and any lower bound for $\rho_{s}(K)$ gives an upper bound of $\delta(K)$, provided we know $f_{\mathrm{D} K}\left(u_{K}\right) V(K) / V\left(K \mid u_{K}^{\perp}\right)$. Namely by definition 2.1.4 we have

$$
\rho_{c}(K)^{1-d} \frac{f_{\mathrm{D} K}\left(u_{K}\right) V(K)}{2 V\left(K \mid u_{K}^{\perp}\right)} \leq \delta(K) \leq \rho_{s}(K)^{1-d} \frac{f_{\mathrm{D} K}\left(u_{K}\right) V(K)}{2 V\left(K \mid u_{K}^{\perp}\right)} .
$$

In general it is hard to determine $f_{\mathrm{D} K}\left(u_{K}\right) V(K) / V\left(K \mid u_{K}^{\perp}\right)$, but from $V(K) \leq$ $2 V\left(K \mid u^{\perp}\right)\left(f_{\mathrm{D} K}(u)\right)^{-1} \leq d V(K), u \in S^{d-1}$, (cf. [Gri85]) we obtain the estimate:

\section{Corollary 2.1.1}

$$
\rho_{c}(K)^{1-d} \frac{1}{d} \leq \delta(K) \leq \rho_{s}(K)^{1-d}
$$

In the special case $K=B^{d}(2.1 .6)$ becomes:

\section{Corollary 2.1.2}

$$
\frac{\kappa_{d}}{2 \kappa_{d-1}}\left(\rho_{c}\left(B^{d}\right)\right)^{1-d} \leq \delta\left(B^{d}\right) \leq \frac{\kappa_{d}}{2 \kappa_{d-1}}\left(\rho_{s}\left(B^{d}\right)\right)^{1-d} .
$$


In section 2.3 we prove $\rho_{c}(K) \leq 2$ for $K \in \mathcal{K}_{0}^{d}$ and $\rho_{c}(K) \leq d+1$ for arbitrary convex bodies $K \in \mathcal{K}^{d}$. Further, in section 2.4 we establish a lower bound for $\rho_{s}(K), K \in \mathcal{K}^{d}$, only depending on the dimension. In chapter 3 we show that for each $\epsilon>0$ there exists a $d(\epsilon)$ with $\rho_{s}\left(B^{d}\right)>\sqrt{2}-\epsilon$ for all $d \geq d(\epsilon)$. On account of these estimates and the inequality $\sqrt{2 \pi /(d+1)}<\kappa_{d} / \kappa_{d-1}<\sqrt{2 \pi / d}$ (cf. [BGW82]) corollary 2.1.2 yields

$$
\sqrt{\frac{2 \pi}{d+1}} 2^{-d}<\delta\left(B^{d}\right)<\sqrt{\frac{\pi}{d}}(\sqrt{2}-\epsilon)^{-d}, \text { for } d \geq d(\epsilon) .
$$

Observe that the lower bound is of the same order as the best known ones (cf. (1.1.9), (1.1.16)) and the upper bound is asymptotically of the same order as the classical bounds of BLICHFELDT (1.1.12) and Rogers (1.1.13). Though this is much weaker than the best known upper bound for $\delta\left(B^{d}\right)$ (cf. (1.1.15)) it shows that the parameterized densities of finite packings are not only of interest in their own but also give a new approach to the study of infinite packings.

Actually, in chapter 3 we prove that for each $\rho<\sqrt{2}$ there exists a $d(\rho)$ with $\delta_{\rho}\left(B^{d}, n\right)=\delta_{\rho}\left(B^{d}, S_{n}\left(B^{d}\right)\right)$ for all $n \in \mathbb{N}$ and $d \geq d(\rho)$ and in chapter 4 we give the bound $d(1) \leq 45$ which means that the sausage conjecture (1.3.2) is true in dimensions $d \geq 45$. Moreover this result says that in high dimensions sausage configurations are best possible packing sets even for $1<\rho<\sqrt{2}$ and we conjecture:

Strong Sausage Conjecture: For $d \geq 1$

$$
\rho_{s}\left(B^{d}\right)=\rho_{c}\left(B^{d}\right)
$$

This conjecture would imply the equivalence of the two problems of determination of $\delta\left(B^{d}\right)$ and $\rho_{c}\left(B^{d}\right)$ (cf. corollary 2.1.2). Clearly for $d=1$ the conjecture is trivial, for $d=2$ its validity follows from a result due to GroEmer (see section 2.2) and with respect to finite lattice packings (see Chapter 5 ) the conjecture was proved by BETKE for $d=3$ [Bet93].

\subsection{The 2-dimensional case}

In this section we show $\rho_{c}(K)=\rho_{s}(K)=\delta_{1}^{s}(K) / \delta(K)$ for 2-dimensional convex bodies. The proof is based on a result of BÖRÖCZKY, JR. and on result about finite packings of centrally symmetric bodies which in particular generalizes the results of Rogers (1.2.6), Oler (1.2.8) and Groemer (1.2.9) to parameterized densities. Indeed the general result is an easy consequence of OLER's result. 
Theorem 2.2.1 Let $K \in \mathcal{K}_{0}^{2}, n \in \mathbb{N}, C \in \mathcal{P}(K, n)$ and $\rho \geq 0$. Then

$$
V(\operatorname{conv}(C)+\rho K) \geq(n-1) \frac{V(K)}{\delta(K)}+2\left(\rho-\frac{\delta_{1}^{s}(K)}{\delta(K)}\right) V_{1}(\operatorname{conv}(C), K)+\rho^{2} V(K) .
$$

Furthermore, $3 / 4 \leq \delta_{1}^{s}(K) / \delta(K) \leq 1$, with $\delta_{1}^{s}(K) / \delta(K)=1$ iff $K$ is a parallelogram, and $\delta_{1}^{s}(K) / \delta(K)=3 / 4$ iff $K$ is a affinely regular non-degenerate hexagon.

Proof. First we consider the range of $\gamma(K)=\delta_{1}^{s}(K) / \delta(K)$. By (1.1.10) and definition 2.1.4 we get $\gamma(K)=V(H(K)) /\left(2 V\left(K \mid u_{K}^{\perp}\right) / f_{K}\left(u_{K}\right)\right)$. Now the denominator $2 V\left(K \mid u_{K}^{\perp}\right) / f_{K}\left(u_{K}\right)$ is the volume of a minimal circumscribed parallelogram for $K$ and thus $\gamma(K)$ is the ratio of the minimal volume of a circumscribed affine regular hexagon (possibly degenerate) to that of a circumscribed parallelogram. Clearly, $\gamma(K) \leq 1$ and equality holds for a parallelogram. On the other hand $\gamma(K)=1$ implies that $K$ is a parallelogram; otherwise two vertices $x^{1},-x^{1}$ of a minimal circumscribed parallelogram $P(K)$ are not contained in $K$ and thus we can find an affinely non-degenerate hexagon $H$ with $K \subset H \subset P(K)$.

To show the lower bound we may assume that $H(K)$ is non-degenerate. Let $P(H)$ be a minimal circumscribed parallelogram of $H(K)$. It is easy to check that $H(K)$ is a maximal non-degenerate inscribed affinely regular hexagon of $P(H)$ and from this one gets (cf. [GL87], pp. 244)

$$
1=\delta(P(H))=(3 / 4) V(P(H)) / V(H(K)) .
$$

Thus $\gamma(K) \geq V(H(K)) / V(P(H))=3 / 4$ and equality holds if $K$ is a nondegenerate affinely regular hexagon. Now suppose that $\gamma(K)=3 / 4$ and without loss of generality we may assume that $H(K)$ is a regular hexagon. Let $x^{1}$ be a vertex of $H(K)$ not contained in $K$ and let $P_{1}, P_{2}$ be minimal circumscribed parallelograms for $K, H(K)$, respectively, with edges parallel to conv $\left\{0, x^{1}\right\}$ and $\left(\operatorname{conv}\left\{0, x^{1}\right\}\right)^{\perp}$. Since $V(H(K)) / V\left(P_{2}\right)=3 / 4$ we see that $V(H(K)) / V\left(P_{1}\right)>$ $3 / 4$.

For the proof of inequality (2.2.1) we may assume $n>1$. Combination of (2.1.2) and OLER's result (1.2.8) yields

$$
\begin{aligned}
& V(\operatorname{conv}(C)+\rho K)=V(\operatorname{conv}(C))+2 \rho V_{1}(\operatorname{conv}(C), K)+\rho^{2} V(K) \\
\geq & (n-1) \frac{V(K)}{\delta(K)}+2\left(\rho-\frac{V(K)}{\delta(K)} \frac{F_{2 K}(\operatorname{conv}(C))}{4 V_{1}(\operatorname{conv}(C), K)}\right) V_{1}(\operatorname{conv}(C), K)+\rho^{2} V(K) .
\end{aligned}
$$

It remains to show $V(K) F_{2 K}(\operatorname{conv}(C)) /\left(\delta(K) 4 V_{1}(\operatorname{conv}(C), K)\right) \leq \gamma(K)$. This follows immediately from an inequality by OLER (cf. [Ole61] ineq. (6), p.48), but for completeness' sake we give a short proof.

Let $x^{1}, \ldots, x^{k}$ denote the vertices of $\operatorname{conv}(C)$ such that $\operatorname{conv}\left\{x^{i}, x^{i+1}\right\}$ is an edge of $\operatorname{conv}(C), 1 \leq i \leq k$, with $x^{k+1}=x^{1}$. The outward unit normal vector of 
the edge $\operatorname{conv}\left\{x^{i}, x^{i+1}\right\}$ is denoted by $v^{i} \in S^{1}$. Let $u^{i}=\left(x^{i}-x^{i+1}\right) /\left|x^{i}-x^{i+1}\right| \in S^{1}$, $1 \leq i \leq k$, where $|\cdot|$ denotes the usual Euclidean norm with associated inner product $\langle\cdot, \cdot\rangle$. Finally, we denote by $h_{K}: S^{1} \rightarrow \mathbb{R}$ the support function of $K$, i.e. $h_{K}(u)=\max \{\langle x, u\rangle: x \in K\}$. Then (cf. [BF34], [SY93])

$$
2 V_{1}(\operatorname{conv}(C), K)=\sum_{i=1}^{k}\left|x^{i}-x^{i+1}\right| h_{K}\left(v^{i}\right) .
$$

Since $K$ is centrally symmetric we obtain $h_{K}\left(v^{i}\right)=V\left(K \mid\left(u^{i}\right)^{\perp}\right) / 2$, and thus

$$
\begin{aligned}
\frac{F_{2 K}(\operatorname{conv}(C))}{2 V_{1}(\operatorname{conv}(C), K)} & =\frac{\sum_{i=1}^{k} f_{2 K}\left(x^{i}-x^{i+1}\right)}{\sum_{i=1}^{k}\left|x^{i}-x^{i+1}\right| h_{K}\left(v^{i}\right)} \\
& =\frac{\sum_{i=1}^{k}\left|x^{i}-x^{i+1}\right| f_{K}\left(u^{i}\right)}{\sum_{i=1}^{k}\left|x^{i}-x^{i+1}\right| V\left(K \mid\left(u^{i}\right)^{\perp}\right)} \leq \frac{f_{K}\left(u_{K}\right)}{V\left(K \mid u_{K}^{\perp}\right)}
\end{aligned}
$$

On account of the definitions of $\delta_{1}^{s}(K)$ and $\gamma(K)$ this shows

$$
\frac{V(K) F_{2 K}(\operatorname{conv}(C))}{\delta(K) 4 V_{1}(\operatorname{conv}(C), K)} \leq \gamma(K)
$$

Remark. For $\rho=1$ and without the summand $V_{1}(\operatorname{conv}(C), K)$ the inequality (2.2.1) becomes Rogers' inequality (1.2.6). Furthermore, by (2.2.3) we have $2 V_{1}\left(\operatorname{conv}(C), B^{2}\right)=F(\operatorname{conv}(C))$ and for $\rho=0, K=B^{2}(2.2 .1)$ is equivalent to Groemer's result (1.2.9). Obviously for $\rho=0$ (2.2.1) is of the same type as OLER's result (1.2.8), but if we do not have equality in (2.2.4), then OLER's result gives a better lower bound for $V(\operatorname{conv}(C))$.

Now we can apply theorem 2.2.1 to the densities $\delta_{\rho}(K, n), \delta_{\rho}(K)$, and obtain:

Theorem 2.2.2 For $K \in \mathcal{K}_{0}^{2}$ and $n \in \mathbb{N}$

$$
\begin{aligned}
& \text { i) } \delta_{\rho}(K, n)=\delta_{\rho}\left(K, S_{n}(K)\right), \quad 0<\rho \leq \frac{\delta_{1}^{s}(K)}{\delta(K)} . \\
& \text { ii) } \delta_{\rho}(K, n) \leq \delta(K)\left(\frac{n}{n-1+\delta(K) \rho^{2}}\right), \quad \frac{\delta_{1}^{s}(K)}{\delta(K)} \leq \rho<\infty . \\
& \text { iii) } \delta_{\rho}(K)=\delta_{\rho}^{s}(K) \text { for } 0<\rho \leq \frac{\delta_{1}^{s}(K)}{\delta(K)} \text { and } \delta_{\rho}(K)=\delta(K) \text { else. }
\end{aligned}
$$

Proof. Let $\rho=\gamma(K)=\delta_{1}^{s}(K) / \delta(K)$ and $C \in \mathcal{P}(K, n)$. By (2.2.1) we get

$$
\begin{aligned}
& V(\operatorname{conv}(C)+\gamma(K) \cdot K) \geq(n-1) \frac{V(K)}{\delta(K)}+\gamma(K)^{2} V(K) \\
& \quad=(n-1) \frac{V(K)}{\delta_{1}^{s}(K)} \gamma(K)+\gamma(K)^{2} V(K)=V\left(\operatorname{conv}\left(S_{n}(K)\right)+\gamma(K) K\right) .
\end{aligned}
$$


So $\delta_{\gamma(K)}(K, n)=\delta_{\gamma(K)}\left(K, S_{n}(K)\right)$ for each $n$, and by theorem 2.1.2 iv) this gives i). For $\gamma(K) \leq \rho<\infty(2.2 .1)$ yields $V(\operatorname{conv}(C)+\rho K) \geq(n-1) V(K) / \delta(K)+\rho^{2} V(K)$ and thus we get ii). Obviously, iii) follows from i) and ii).

From iii) of the above theorem we may deduce:

Corollary 2.2.1 For $K \in \mathcal{K}_{0}^{2}$ holds

$$
\frac{3}{4} \leq \rho_{s}(K)=\frac{\delta_{1}^{s}(K)}{\delta(K)}=\rho_{c}(K) \leq 1
$$

Observe that by theorem 2.2.2 i) and theorem 2.1.2 iv) a densest sausage configuration of $K$ is the "only" best possible packing set with respect to $\delta_{\rho}(K, n)$ for $0<\rho<\delta_{1}^{s}(K) / \delta(K)$. But for $\rho=\delta_{1}^{s}(K) / \delta(K)$ various optimal configurations are possible as figure 2.2.1 shows for $K=B^{2}, \delta_{1}^{s}\left(B^{2}\right) / \delta\left(B^{2}\right)=\sqrt{3} / 2$ and $n=7$ (cf. [Gro60], [Weg86]).

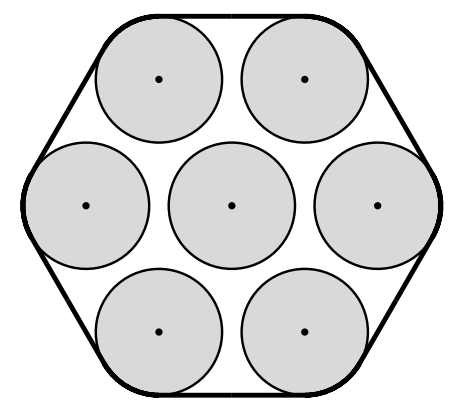

$\delta_{\sqrt{3} / 2}\left(B^{2}, C\right)=\frac{7 \pi}{6 \sqrt{3}+12 \sqrt{3} / 2+\pi(\sqrt{3} / 2)^{2}} \sim 0.950312$.

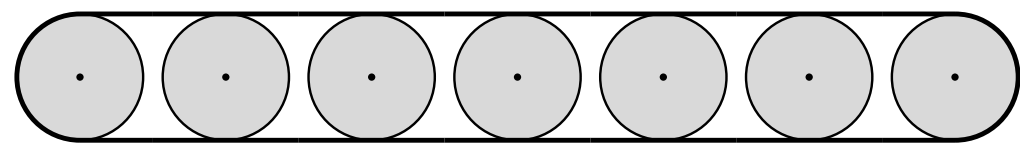

$\delta_{\sqrt{3} / 2}\left(B^{2}, S_{7}\left(B^{2}\right)\right)=\delta_{\sqrt{3} / 2}\left(B^{2}, C\right)$.

Figure 2.2.1.

Unfortunately, for arbitrary convex bodies in the plane we do not have a result of the type of theorem 2.2.1. However, recently BöröczKY, JR. (personal communication) showed that for each $K \in \mathcal{K}^{2}$ the sausage parameter and the critical parameter are the same. As an immediate consequence we get: 
Corollary 2.2.2 For every $K \in \mathcal{K}^{2}$ one has

$$
\frac{3}{4} \leq \rho_{s}(K)=\rho_{s}(\mathrm{D} K)=\frac{\delta_{1}^{s}(K)}{\delta(K)}=\frac{\delta_{1}^{s}(\mathrm{D} K)}{\delta(\mathrm{D} K)}=\rho_{c}(\mathrm{D} K)=\rho_{c}(K) \leq 1 .
$$

Proof. By (2.1.6) we have $\rho_{s}(K) \leq \delta_{1}^{s}(K) / \delta(K) \leq \rho_{c}(K)$. So the result of BÖRÖCZKY, JR. gives $\rho_{s}(K)=\delta_{1}^{s}(K) / \delta(K)=\rho_{c}(K)$. In view of corollary 2.2.1 it remains to show

$$
\frac{\delta_{1}^{s}(K)}{\delta(K)}=\frac{\delta_{1}^{s}(\mathrm{D} K)}{\delta(\mathrm{D} K)}
$$

Now, since the breadths of the bodies $K$ and $\mathrm{D} K$ are equal for each direction $v \in S^{1}$ (cf. [BF34]) we have $V\left(\mathrm{D} K \mid v^{\perp}\right)=V\left(K \mid v^{\perp}\right)$ for $v \in S^{1}$. Hence a sausage configuration of $\mathrm{D} K$ in direction $u_{K}$ is a densest sausage configuration of $D K$. Thus (cf. (1.1.11))

$$
\frac{\delta_{1}^{s}(K) \delta(\mathrm{D} K)}{\delta_{1}^{s}(\mathrm{D} K) \delta(K)}=\frac{V\left(\mathrm{D} K \mid u_{K}^{\perp}\right)}{V\left(K \mid u_{K}^{\perp}\right)}=1
$$

In the next two sections we state bounds for the critical and sausage parameter that hold in all dimensions.

\subsection{An upper bound for the critical parameter}

The main result of this section is:

Theorem 2.3.1 Let $K \in \mathcal{K}^{d}$ and $\rho \in \mathbb{R}^{>0}$ such that $\operatorname{int}(K) \cap \operatorname{int}(y+K)=\emptyset$ holds for all $y \notin \rho K$. Then for each $n \in \mathbb{N}$

$$
\delta_{\rho}(K, n) \leq \delta(K)
$$

Thus for such a $\rho$ the density of a densest infinite packing is an upper bound for the density of a finite packing. Hence it follows $\delta_{\rho}(K) \leq \delta(K)$. On account of theorem $2.1 .1 \mathrm{vi}$ ) we have $\delta_{\rho}(K)=\delta(K)$ for any parameter $\rho$ as in theorem 2.3.1. This implies that a $\rho$ with this "non-intersecting" property is an upper bound for the critical parameter. It is easy to see that for $K \in \mathcal{K}_{0}^{d}$ this property is equivalent to $\rho \geq 2$, whereas for an arbitrary convex body such a $\rho$ may depend on the shape of the body. But in this case we obtain a bound only depending on the dimension.

Corollary 2.3.1

$$
\rho_{c}(K) \leq\left\{\begin{array}{l}
2: \text { for } K \in \mathcal{K}_{0}^{d}, \\
d+1: \text { for general } K
\end{array}\right.
$$


Proof. Let $K \in \mathcal{K}_{0}^{d}, \rho \geq 2$, and $y \notin \rho K$. Assume $\operatorname{int}(K) \cap \operatorname{int}(y+K) \neq \emptyset$. Then there exist $x, z \in \operatorname{int}(K)$ with $x=y+z$ and thus $y \in K+(-K)=2 K$, which contradicts the choice of $\rho$.

Let $K \in \mathcal{K}^{d}$. Since $\delta_{\rho}(K, n)$ is invariant with respect to translations of $K$ (theorem 2.1.1 ii)) we may assume $K+(-K) \subset(d+1) K$ (cf. [Rog64]). As above we obtain $\operatorname{int}(K) \cap \operatorname{int}(y+K)=\emptyset$ for all $y \notin(d+1) K$.

The proof of theorem 2.3.1 is based on the following idea: Suppose that $C$ is a finite packing set of $K$ with $\delta_{\rho}(K, C)>\delta(K)$. Then a packing lattice $\Lambda$ of $\operatorname{conv}(C)+\rho K$ with elementary cell $Z$ is chosen. For every $x \in Z$ the lattice packing $L(\operatorname{conv}(C)+\rho K+x)=\{(\operatorname{conv}(C)+\rho K+x)+g: g \in \Lambda\}$ is superposed on a densest infinite packing $\left\{K+a: a \in C_{\infty}\right\}$ with density $\delta(K)$. Also all $K+a$, $a \in C_{\infty}$ that meet $L((\operatorname{conv}(C)+\rho K)+x)$ are deleted.

A standard averaging argument with respect to $x$ shows the existence of an infinite packing of translates of $K$ with density $>\delta(K)$, which contradicts the definition of $\delta(K)$. Hence $\delta_{\rho}(K, C) \leq \delta(K)$. The proof gives a careful analysis of this idea.

Proof of Theorem 2.3.1. Assume there exists $K \in \mathcal{K}^{d}$ with $0 \in \operatorname{int}(K), \rho \in \mathbb{R}^{>0}$ satisfying the assumption and an integer $n$ with $\delta_{\rho}(K, n)>\delta(K)$. Then there is a $C \in \mathcal{P}(K, n)$ and an $\epsilon>0$ with

$$
V(\operatorname{conv}(C)+\rho K)=n \cdot \frac{V(K)}{\delta(K)}-\epsilon
$$

Let $\Lambda$ be a packing lattice of $\operatorname{conv}(C)+\rho K$, i.e., $\Lambda \in \mathcal{P}^{*}(\operatorname{conv}(C)+\rho K)$. We may assume that $\operatorname{conv}(C)+\rho K$ is contained in a fixed elementary cell $Z$ of $\Lambda$. From (2.3.1) follows

$$
\left(1-\frac{V(\operatorname{conv}(C)+\rho K)}{\operatorname{det}(\Lambda)}\right) \frac{\operatorname{det}(\Lambda)}{\operatorname{det}(\Lambda)+\epsilon}+\frac{n V(K) / \delta(K)}{\operatorname{det}(\Lambda)+\epsilon}=1 .
$$

Multiplication with $\delta(K)$ yields

$$
\left(1-\frac{V(\operatorname{conv}(C)+\rho K)}{\operatorname{det}(\Lambda)}\right) \delta(K) \frac{\operatorname{det}(\Lambda)}{\operatorname{det}(\Lambda)+\epsilon}+\frac{n V(K)}{\operatorname{det}(\Lambda)+\epsilon}=\delta(K) .
$$

Apparently there is a constant $\mu$ only depending on $Z$ such that for every $\lambda>0$ there is a subset $L_{\lambda} \subset \Lambda$ such that $W_{\lambda}^{d}+Z \subset L_{\lambda}+Z$ and $L_{\lambda}+2 Z \subset W_{\lambda+\mu}^{d}$.

By the definition of $\delta(K)$ (cf. (1.1.1)) for every $\lambda>0$ there exists a set $C_{m(\lambda)} \in \mathcal{P}(K, m(\lambda))$ such that $C_{m(\lambda)}+K \subset W_{\lambda}^{d}$ and

$$
\lim _{\lambda \rightarrow \infty} \frac{m(\lambda) V(K)}{V\left(W_{\lambda}^{d}\right)}=\delta(K)
$$


Obviously $\lim _{\lambda \rightarrow \infty} V\left(W_{\lambda+\mu}^{d}\right) / V\left(W_{\lambda}^{d}\right)=1$, so there exists a $\zeta>0$ and a set $C_{m(\zeta)} \in$ $\mathcal{P}(K, m(\zeta))$ with $C_{m(\zeta)}+K \subset W_{\zeta}^{d}$ such that

$$
\delta(K) \frac{\operatorname{det}(\Lambda)}{\operatorname{det}(\Lambda)+\epsilon}<\frac{m(\zeta) V(K)}{V\left(W_{\zeta+\mu}^{d}\right)} \text { and } \quad \frac{n V(K)}{\operatorname{det}(\Lambda)+\epsilon}<\frac{n V(K) \#\left(L_{\zeta}\right)}{V\left(W_{\zeta+\mu}^{d}\right)}
$$

For every $x \in Z$ we construct a finite packing $C_{n(x)} \in \mathcal{P}(K, n(x))$ - for a suitable $n(x) \in \mathbb{N}$ - with $C_{n(x)}+K \subset W_{\zeta+\mu}^{d}$ in the following way:

$$
C_{n(x)}=\left\{x+L_{\zeta}+C\right\} \cup\left\{y \in C_{m(\zeta)}: y \notin x+L_{\zeta}+(\operatorname{conv}(C)+\rho K)\right\} .
$$

The choice of $\rho$ guarantees that $C_{n(x)}$ is a packing. While it is difficult to determine the cardinality $n(x)$ of $C_{n(x)}$ for fixed $x$ it is easy to calculate $\int_{x \in Z} n(x) d x$. To this end for every $y \in C_{m(\zeta)}$ let $\chi_{y}(x)=1$ for $y \notin x+L_{\zeta}+(\operatorname{conv}(C)+\rho K)$ and $\chi_{y}(x)=0$ else. Then

$$
\begin{aligned}
\int_{x \in Z} n(x) d x & =\int_{x \in Z}\left(n \#\left(L_{\zeta}\right)+\sum_{y \in C_{m(\zeta)}} \chi_{y}(x)\right) d x \\
& =n \operatorname{det}(\Lambda) \#\left(L_{\zeta}\right)+m(\zeta)(\operatorname{det}(\Lambda)-V(\operatorname{conv}(C)+\rho K)) .
\end{aligned}
$$

So there is a $z \in Z$ with

$$
n(z) \geq m(\zeta)\left(1-\frac{V(\operatorname{conv}(C)+\rho K)}{\operatorname{det}(\Lambda)}\right)+n \#\left(L_{\zeta}\right)
$$

or

$$
\frac{n(z) V(K)}{V\left(W_{\zeta+\mu}^{d}\right)} \geq \frac{m(\zeta) V(K)}{V\left(W_{\zeta+\mu}^{d}\right)}\left(1-\frac{V(\operatorname{conv}(C)+\rho K)}{\operatorname{det}(\Lambda)}\right)+\frac{n V(K) \#\left(L_{\zeta}\right)}{V\left(W_{\zeta+\mu}^{d}\right)}
$$

From (2.3.2) and (2.3.3) follows

$$
\frac{n(z) V(K)}{V\left(W_{\zeta+\mu}^{d}\right)}>\delta(K)
$$

But this contradicts the definition of $\delta(K)$.

\subsection{A lower bound for the sausage parameter}

The purpose of this section is to give a lower bound for the sausage parameter depending on the dimension and the ratio of inradius to circumradius. To this end we denote for $K \in \mathcal{K}^{d}$ by $R(K), r(K)$ the circumradius and inradius, respectively. To get a lower bound for the sausage parameter we prove as in the case of the critical parameter a result about finite packings. 
Theorem 2.4.1 Let $K \in \mathcal{K}^{d}$ and $n \in \mathbb{N}$. Then for $\rho \leq(1 / 32) d^{-1}(r(K) / R(K))$

$$
\delta_{\rho}(K, n)=\delta_{\rho}\left(K, S_{n}(K)\right) .
$$

Thus for $\rho \leq(1 / 32) d^{-1}(r(K) / R(K))$ and every $n \in \mathbb{N}$ a densest sausage arrangement is a best possible arrangement. Obviously, this implies:

Corollary 2.4.1 For every $K \in \mathcal{K}^{d}$ one has

$$
\rho_{s}(K) \geq \frac{1}{32} d^{-1} \frac{r(K)}{R(K)} .
$$

Roughly speaking the idea behind the proof of theorem 2.4.1 is the following: For a given packing set $C=\left\{x^{1}, \ldots, x^{n}\right\}$ we choose $l+1$ affinely independent points $\left\{x^{1}, \ldots, x^{l+1}\right\}$ of $C$ such that for each point $x^{i}$ of $C$ the intersection of $x^{i}+\mathrm{D} K$ with the affine plane $L_{l}$ spanned by $x^{1}, \ldots, x^{l+1}$ is "large" and also the volume of the $l$-simplex with vertices $x^{1}, \ldots, x^{l+1}$ is not too "small". By these two properties we obtain a lower bound of the volume of $\operatorname{conv}(C) \cap L_{l}$ depending on $n, l$ and $r(K)$. Substituting this estimate in a well-known inequality concerning mixed volumes we get

$$
V(\operatorname{conv}(C)+\rho K) \geq \rho^{d-l} n c(l, r(K), R(K)) V\left(K \mid v^{\perp}\right)+\rho^{d} V(K),
$$

where $c(l, r(K), R(K))$ is a constant depending on $l, r(K), R(K)$ and $v$ is a unit vector in the linear space associated to $L_{l}$. Comparing the right hand side with the volume of a sausage arrangement in direction $v$ gives the bound for $\rho$ and $l \geq 2$. The case $l=1$ needs some additional considerations.

Proof of theorem 2.4.1. Let $n \in \mathbb{N}, n>1, K \in \mathcal{K}^{d}, C=\left\{x^{1}, \ldots, x^{n}\right\} \in \mathcal{P}(K, n)$, $\rho \leq(1 / 32) d^{-1}(r(K) / R(K))$ and let $m=\operatorname{dim}(C)$. To simplify the notation we write $R$ instead of $R(K)$. Let $z_{R} \in E^{d}$ with $K \subset z_{R}+R B^{d}$ and let $\bar{r}$ be the inradius of $\mathrm{D} K$. Then

$$
\bar{r} \geq r(K)
$$

The proof now proceeds in four steps:

(1) We construct iteratively from $C$ distances $h_{j}$ and associated subspaces $L_{j}$ as follows: Without loss of generality let $x^{1}=0$ and let the diameter of $C$ be given by the distance of the points $x^{1}, x^{2}$. We set $h_{1}=\left|x^{1}-x^{2}\right| / 2$ and $L_{1}=\operatorname{lin}\left\{x^{1}, x^{2}\right\}$, where $\operatorname{lin}(S)$ (or $\operatorname{lin} S)$ denotes the linear hull of a set $S$. Since $C \in \mathcal{P}(\mathrm{D} K, n)$ we have $h_{1} \geq \bar{r}$. Suppose we have chosen distances $h_{i}$ and linear spaces $L_{i}$, $1 \leq i<m$. Let $h_{i+1}=\max \left\{\operatorname{dist}\left(x^{k}, L_{i}\right): 1 \leq k \leq n\right\}$, where $\operatorname{dist}\left(x^{k}, L_{i}\right)$ denotes the Euclidean distance of $x^{k}$ to $L_{i}$. We may assume $h_{i+1}=\operatorname{dist}\left(x^{i+1}, L_{i}\right)$ and we set $L_{i+1}=\operatorname{lin}\left(L_{i} \cup\left\{x^{i+1}\right\}\right)$. We continue up to $i=m$ and obtain a monotone sequence of distances:

$$
2 h_{1} \geq h_{2} \geq h_{3} \geq \ldots \geq h_{m} \geq 0 \text {. }
$$


For $m=1$ we have $\operatorname{dim}(C)=1$ and thus $V(\operatorname{conv}(C)+\rho K) \geq V\left(\operatorname{conv}\left(S_{n}(K)\right)\right.$ $+\rho K)$. So let $m>1, h_{m+1}=0$ and $l \in\{1, \ldots, m\}$ such that

$$
h_{l}>\bar{r} / \sqrt{2} \text { and } h_{l+1} \leq \bar{r} / \sqrt{2} \text {. }
$$

(2) For $1 \leq j \leq m$ let $C^{j}=L_{j} \cap \operatorname{conv}(C)$. Then $\operatorname{dim}\left(C^{j}\right)=j$ and we have for the mixed volumes (cf. [Sch93])

$$
\left(\begin{array}{l}
d \\
j
\end{array}\right) \rho^{d-j} V_{j}(\operatorname{conv}(C), K) \geq\left(\begin{array}{l}
d \\
j
\end{array}\right) \rho^{d-j} V_{j}\left(C^{j}, K\right)=\rho^{d-j} V\left(C^{j}\right) \cdot V\left(K \mid L_{j}^{\perp}\right) .
$$

Now let $v \in L_{j} \cap S^{d-1}$. Since $K \subset z_{R}+R B^{d}$ we have $V\left(K \mid v^{\perp}\right) \leq \kappa_{j-1} R^{j-1}$ $V\left(K \mid L_{j}^{\perp}\right)$ and so

$$
\left(\begin{array}{l}
d \\
j
\end{array}\right) \rho^{d-j} V_{j}(\operatorname{conv}(C), K) \geq \rho^{d-j} V\left(C^{j}\right) \cdot V\left(K \mid v^{\perp}\right) \kappa_{j-1}^{-1} R^{1-j} .
$$

In the last two steps of the proof we consider the cases $l=1$ and $l>1$.

(3) Let $l=1$. From (2.1.2), (2.1.3) and (2.4.2) we get for $v \in L_{1} \cap S^{d-1}$

$$
\begin{gathered}
V(\operatorname{conv}(C)+\rho K)-V\left(\operatorname{conv}\left(S_{n}(v, K)\right)+\rho K\right) \geq \\
\sum_{j=1}^{2}\left(\begin{array}{c}
d \\
j
\end{array}\right) \rho^{d-j} V_{j}(\operatorname{conv}(C))-2(n-1) \rho^{d-1} V\left(K \mid v^{\perp}\right) / f_{\mathrm{D} K}(v) \geq \\
\rho^{d-2} V\left(C^{2}\right) \cdot V\left(K \mid v^{\perp}\right) \frac{1}{2} R^{-1}+\rho^{d-1}\left(V\left(C^{1}\right)-2(n-1) / f_{\mathrm{D} K}(v)\right) V\left(K \mid v^{\perp}\right) .
\end{gathered}
$$

Since $h_{j} \leq h_{2} \leq \bar{r} / \sqrt{2}$ for $j>1, \bar{r} B^{d} \subset \mathrm{DK}$ and $\left(1 / f_{\mathrm{DK}}(v)\right) B^{d} \cap L_{1} \subset$ $\mathrm{D} K$ the intersection of $x^{i}+\mathrm{D} K$ with $L_{1}$ contains a line segment of length $2\left(1-h_{2} / \bar{r}\right) / f_{\mathrm{D} K}(v), 1 \leq i \leq n$. Furthermore, since $C$ is a packing set with respect to DK (cf. (1.1.6)) any two of these segments have disjoint interiors and from the definition of $L_{1}$ we get $V\left(C^{1}\right) \geq 2(n-1)\left(1-h_{2} / \bar{r}\right) / f_{\mathrm{D} K}(v)$ and thus

$$
V\left(C^{2}\right) \geq V\left(\operatorname{conv}\left(C_{1} \cup\left\{x^{2}\right\}\right)\right) \geq h_{2}(n-1) \frac{1-h_{2} / \bar{r}}{f_{\mathrm{D} K}(v)} .
$$

Now $h_{2} / \bar{r} \leq 1 / \sqrt{2}$ and hence

$$
\begin{aligned}
& V(\operatorname{conv}(C)+\rho K)-V\left(\operatorname{conv}\left(S_{n}(v, K)\right)+\rho K\right) \geq \\
& \rho^{d-2} \frac{2(n-1) V\left(K \mid v^{\perp}\right)}{f_{\mathrm{D} K}(v) \bar{r}} h_{2}\left(\frac{1}{4}(1-1 / \sqrt{2}) \frac{\bar{r}}{R}-\rho\right) .
\end{aligned}
$$

By the choice of $\rho$ we obtain $V(\operatorname{conv}(C)+\rho K)-V\left(\operatorname{conv}\left(S_{n}(v, K)\right)+\rho K\right) \geq 0$.

(4) Let $l>1$. The construction of $C^{l}$ implies $V\left(C^{l}\right) \geq(2 / l !) \prod_{i=1}^{l} h_{i}$. Let $Q^{l}$ be the box with edge lengths $2 h_{1}, 2 h_{2}, \ldots, 2 h_{l}$ circumscribed to $C^{l}$ such that its facets have distance $h_{i}$ from $L_{i-1}, 2 \leq i \leq l$. Clearly,

$$
V\left(Q^{l}\right)=2^{l} \prod_{i=1}^{l} h_{i} \leq l ! 2^{l-1} V\left(C^{l}\right) .
$$


Let $\bar{x}^{j}, 1 \leq j \leq n$, denote the projection of $x^{j}$ onto $L_{l}$ and let $v \in L_{l} \cap S^{d-1}$ be chosen such that $f_{\mathrm{D} K}(v)=\max \left\{f_{\mathrm{D} K}(u): u \in L_{l} \cap S^{d-1}\right\}$. Since $h_{l+1} \leq \bar{r} / \sqrt{2}$ the intersection of $x^{i}+\mathrm{D} K$ with $L_{l}$ contains an $l$-dimensional ball of radius $\gamma>0$, say, and center $\bar{x}^{i}, 1 \leq i \leq n$. Any two of these balls have disjoint interiors and are contained in a box with the same center as $Q^{l}$ and edge lengths $2\left(h_{1}+\gamma\right), 2\left(h_{2}+\gamma\right), \ldots, 2\left(h_{l}+\gamma\right)$. Thus $n \kappa_{l} \gamma^{l}<\prod_{i=1}^{l}\left(1+\gamma / h_{i}\right) V\left(Q^{l}\right)$ or with (2.4.3):

$$
2 n \kappa_{l} \leq V\left(C^{l}\right) l ! 2^{l} \cdot \prod_{i=1}^{l}\left(\frac{1}{\gamma}+\frac{1}{h_{i}}\right) .
$$

By the choice of $v$ we have $\left(1 / f_{\mathrm{D} K}(v)\right)\left(B^{d} \cap L_{l}\right) \subset \mathrm{D} K$ and hence $\gamma \geq(1-$ $\left.h_{l+1} / \bar{r}\right) / f_{\mathrm{D} K}(v)$. Together with $h_{1} \geq 1 / f_{\mathrm{D} K}(v)$ we obtain

$$
\frac{1}{\gamma}+\frac{1}{h_{1}} \leq f_{\mathrm{DK}}(v) \frac{2 \sqrt{2}-1}{\sqrt{2}-1}
$$

Hence

$$
\frac{2 n}{f_{\mathrm{D} K}(v)} \kappa_{l} \leq V\left(C^{l}\right) l ! 2^{l} \cdot\left(\frac{2 \sqrt{2}-1}{\sqrt{2}-1}\right) \prod_{i=2}^{l}\left(\frac{1}{\gamma}+\frac{1}{h_{i}}\right) .
$$

Since $\bar{r} B^{d} \subset \mathrm{D} K$ we also have $\gamma \geq\left(\bar{r}^{2}-h_{l+1}^{2}\right)^{1 / 2}$ and on account of $h_{i}>\bar{r} / \sqrt{2}$, $1 \leq i \leq l$, we get

$$
\begin{aligned}
\frac{2 n}{f_{\mathrm{D} K}(v)} \kappa_{l} & \leq V\left(C^{l}\right) l ! 2^{l} \cdot\left(\frac{2 \sqrt{2}-1}{\sqrt{2}-1}\right)(2 \sqrt{2})^{l-1} \bar{r}^{1-l} \\
& =V\left(C^{l}\right) l ! 2^{(5 l-3) / 2} \cdot \bar{r}^{1-l}\left(\frac{2 \sqrt{2}-1}{\sqrt{2}-1}\right) .
\end{aligned}
$$

Now we use this lower bound of $V\left(C^{l}\right)$ in formula (2.4.2) and by (2.1.2), (2.1.3), (2.4.1) and the choice of $\rho$ we find

$$
\begin{gathered}
V(\operatorname{conv}(C)+\rho K)-V\left(\operatorname{conv}\left(S_{n}(v, K)\right)+\rho K\right) \geq \\
\frac{2(n-1) V\left(K \mid v^{\perp}\right)}{f_{\mathrm{D} K}(v)} \rho^{d-l}\left(\frac{r(K)}{R}\right)^{l-1}\left(\frac{\kappa_{l}}{\kappa_{l-1}}(l !)^{-1} 2^{(3-5 l) / 2}\left(\frac{2 \sqrt{2}-1}{\sqrt{2}-1}\right)^{-1}-(32 d)^{1-l}\right) .
\end{gathered}
$$

Let the term in the last brackets be denoted by $g(l, d)$. It remains to show that $g(l, d) \geq 0$ for $2 \leq l \leq d$. Obviously $g(l, d) \geq g(l, l)$ and it can easily be checked that $g(2,2)>0$. So let $l \geq 3$. With $(2 \sqrt{2}-1) /(\sqrt{2}-1)<9 / 2, \kappa_{l} / \kappa_{l-1}>$ $(2 \pi /(l+1))^{1 / 2}$ (cf. [BGW82]) and StiRLING's formula $l !<(l / e)^{l}(2 \pi(l+1))^{1 / 2}$ we get

$$
g(l, d) \geq g(l, l)>\left(\frac{1}{l} \frac{e}{4 \sqrt{2}}\right)^{l-1}\left(\frac{e}{9(l+1) l}-\left(\frac{\sqrt{2}}{8 e}\right)^{l-1}\right) .
$$

From this the assertion follows, since $h(l):=(l(l+1))^{-1 /(l-1)}$ is monotonely increasing and the difference in the large brackets is positive for $l=3$. 
Let us remark that the constant factor in the upper bound of $\rho_{s}(K)$ can easily be improved, but clearly this factor has non influence on the asymptotic behavior of the bound as $d$ tends to infinity.

By JoHN's theorem [Joh48] we can always achieve by an affine transformation $A$ that the ratio $r(A K) / R(A K)$ is bounded from below by $d^{-1}$ for $K \in \mathcal{K}^{d}$. For $K \in \mathcal{K}_{0}^{d}$ one can even achieve $r(A K) / R(A K) \geq d^{-1 / 2}$. Since $\delta_{\rho}(K, n)$ is invariant under affine mappings we can deduce from theorem 2.4.1:

Corollary 2.4.2 Let $n \in \mathbb{N}$.

$$
\begin{aligned}
& \delta_{\rho}(K, n)=\delta_{\rho}\left(K, S_{n}(K)\right), \quad \text { for } \rho \leq(1 / 32) d^{-2} \text { and } K \in \mathcal{K}^{d} \\
& \delta_{\rho}(K, n)=\delta_{\rho}\left(K, S_{n}(K)\right), \quad \text { for } \rho \leq(1 / 32) d^{-3 / 2} \text { and } K \in \mathcal{K}_{0}^{d} .
\end{aligned}
$$

The bound of theorem 2.4.1 has the disadvantage that it tends to 0 independently of the shape of the body as $d$ tends to infinity. In the next chapter we state another lower bound for $\rho_{s}(K), K \in \mathcal{K}_{0}^{d}$, which depends more on the shape of the given body. In particular we will prove $\lim _{d \rightarrow \infty} \rho_{s}\left(B^{d}\right) \geq \sqrt{2}$, but unfortunately this bound gives by JoHN's theorem a much worse estimate than in the above corollary. However, we believe:

Conjecture 2.4.1 There exists a constant $c \in \mathbb{R}^{>0}$ such that for $K \in \mathcal{K}_{0}^{d}$

$$
\rho_{s}(K) \geq c
$$




\section{Sausages are good packings}

In the last section we have seen that sausage configurations yield best possible finite packings with respect to $\delta_{\rho}(K, n)$ if $\rho$ is small. In the first section of this chapter we show that for the $d$-dimensional unit ball sausages are even best possible for every $\rho<\sqrt{2}$ provided the dimension is large enough. In particular this verifies the sausage conjecture in high dimensions.

The second section deals with arbitrary centrally symmetric convex bodies. We transfer the method of the proof for the ball to symmetric bodies and we obtain a result similar to theorem 2.4.1. This result has the advantage that it gives for $K=B^{d}$ the bound mentioned above. But as the proofs are rather technical we start with the case of a ball, which is easier to visualize.

Section 3.1 is a combination of results from [BHW94a] and [BHW94b]. Section 3.2 is part of [BHW94a].

\subsection{Finite ball packings}

The purpose of this section is to prove the following theorem:

Theorem 3.1.1 For every $\rho<\sqrt{2}$ exists a sausage dimension $d(\rho)$ such that for $d \geq d(\rho), n \in \mathbb{N}$ and $C \in \mathcal{P}\left(B^{d}, n\right)$

$$
V\left(\operatorname{conv}(C)+\rho B^{d}\right) \geq V\left(\operatorname{conv}\left(S_{n}\left(B^{d}\right)\right)+\rho B^{d}\right)
$$

with equality if and only if $C$ is a sausage configuration.

So for $d \geq d(\rho)$ a sausage arrangement is the "only" best possible packing of $n$ balls with respect to the density $\delta_{\rho}\left(B^{d}, C\right)$. In the next chapter we will give an estimate for $d(1)$. Clearly the theorem implies:

Corollary 3.1.1 $\liminf _{d \rightarrow \infty} \rho_{s}\left(B^{d}\right) \geq \sqrt{2}$.

Before we go into details we give a brief sketch of the underlying ideas. Recall that for $n \in \mathbb{N}$ and $\rho>0$ (cf. (2.1.3)):

$$
V\left(\operatorname{conv}\left(S_{n}\left(B^{d}\right)\right)+\rho B^{d}\right)=2(n-1) \kappa_{d-1} \rho^{d-1}+\kappa_{d} \rho^{d},
$$

where $\kappa_{j}$ denotes the $j$-dimensional volume of the $j$-dimensional unit ball. 
In order to prove inequality (3.1.1) we use a local approach, i.e., for a packing set $C=\left\{x^{1}, \ldots, x^{n}\right\}$ we consider the associated Dirichlet-VoronoÏ cells (DVcells, for short) $H^{i}(C), 1 \leq i \leq n$, given by

$$
\begin{aligned}
H^{i}(C) & =\left\{x \in E^{d}:\left|x-x^{i}\right| \leq\left|x-x^{j}\right|, 1 \leq j \leq n\right\} \\
& =\left\{x \in E^{d}: 2\left\langle x, x^{j}-x^{i}\right\rangle \leq\left|x^{j}\right|^{2}-\left|x^{i}\right|^{2}, 1 \leq j \leq n\right\}
\end{aligned}
$$

and the parts of $\operatorname{conv}(C)+\rho B^{d}$ belonging to $H^{i}(C)$ :

$$
D_{\rho}\left(H^{i}(C)\right)=H^{i}(C) \cap\left(\operatorname{conv}(C)+\rho B^{d}\right) .
$$

Obviously we have

$$
V\left(\operatorname{conv}(C)+\rho B^{d}\right)=\sum_{i=1}^{n} V\left(D_{\rho}\left(H^{i}(C)\right)\right)
$$

For a sausage configuration we have $D_{\rho}\left(H^{i}\left(S_{n}\left(B^{d}\right)\right)\right)=2 \kappa_{d-1} \rho^{d-1}, i=2, \ldots, n-$ 1 , and $D_{\rho}\left(H^{1}\left(S_{n}\left(B^{d}\right)\right)\right)=D_{\rho}\left(H^{n}\left(S_{n}\left(B^{d}\right)\right)=\kappa_{d-1} \rho^{d-1}+\kappa_{d} \rho^{d} / 2\right.$. Thus it suffices to prove

$$
V\left(D_{\rho}\left(H^{i}(C)\right)\right) \geq \begin{cases}2 \kappa_{d-1} \rho^{d-1} & : \text { for } n-2 \text { sets } \\ \kappa_{d-1} \rho^{d-1}+\kappa_{d} \rho^{d} / 2 & : \text { for the remaining } 2 \text { sets. }\end{cases}
$$

Figure 3.1.1 shows the sets $H^{i}=H^{i}(C)$ and the associated sets $D^{i}=D_{1}\left(H^{i}(C)\right)$ for a "hexagonal" and a sausage configuration of seven points.

The basic idea for the proof of (3.1.4) is to measure the local deviation of $C$ from a sausage configuration "in" the DV-cell $H^{i}(C)$ by a certain angle $\phi^{i}$. This angle gives information about the situation in the DV-cell and with help of this angle we divide the set $D_{\rho}\left(H^{i}(C)\right)$ in a sausage part and a part which is the Cartesian product of a 2 -dimensional set and essentially a $(d-2)$-ball of radius $\rho$.

The sausage part is of size $\kappa_{d-1} \rho^{d-1}\left(2-\right.$ const $\left._{1} \phi^{i}\right)$ and the other part is of size const $_{2} \kappa_{d-2} \rho^{d-2} \phi^{i}$, where const ${ }_{1}$ and const $_{2}$ are constants independent of $d$. Now (3.1.4) follows from $\kappa_{d-2} / \kappa_{d-1} \rightarrow \infty$ as $d \rightarrow \infty$.

In the following we state several lemmas which give estimates for the volume of $D_{\rho}\left(H^{i}(C)\right)$ with respect to $\phi^{i}$. To this end let $C=\left\{x^{1}, \ldots, x^{n}\right\} \in \mathcal{P}\left(B^{d}, n\right)$, $n>1$, be an arbitrary but fixed arrangement. To avoid getting too many indices we restrict our study to a fixed DV-cell, say $H=H^{n}(C)$, with associated set $D_{\rho}=D_{\rho}\left(H^{n}(C)\right)$. Furthermore, we may assume $x^{n}=0$. For this DV-cell we now define the angle $\phi=\phi^{n}$ and some additional tools.

Definition 3.1.1 Let $y^{j}=x^{j} /\left|x^{j}\right|, 1 \leq j \leq n-1$, and

$$
\phi=\max \left\{\arccos \left(\left|\left\langle y^{k}, y^{l}\right\rangle\right|\right): 1 \leq k, l \leq n-1\right\}
$$



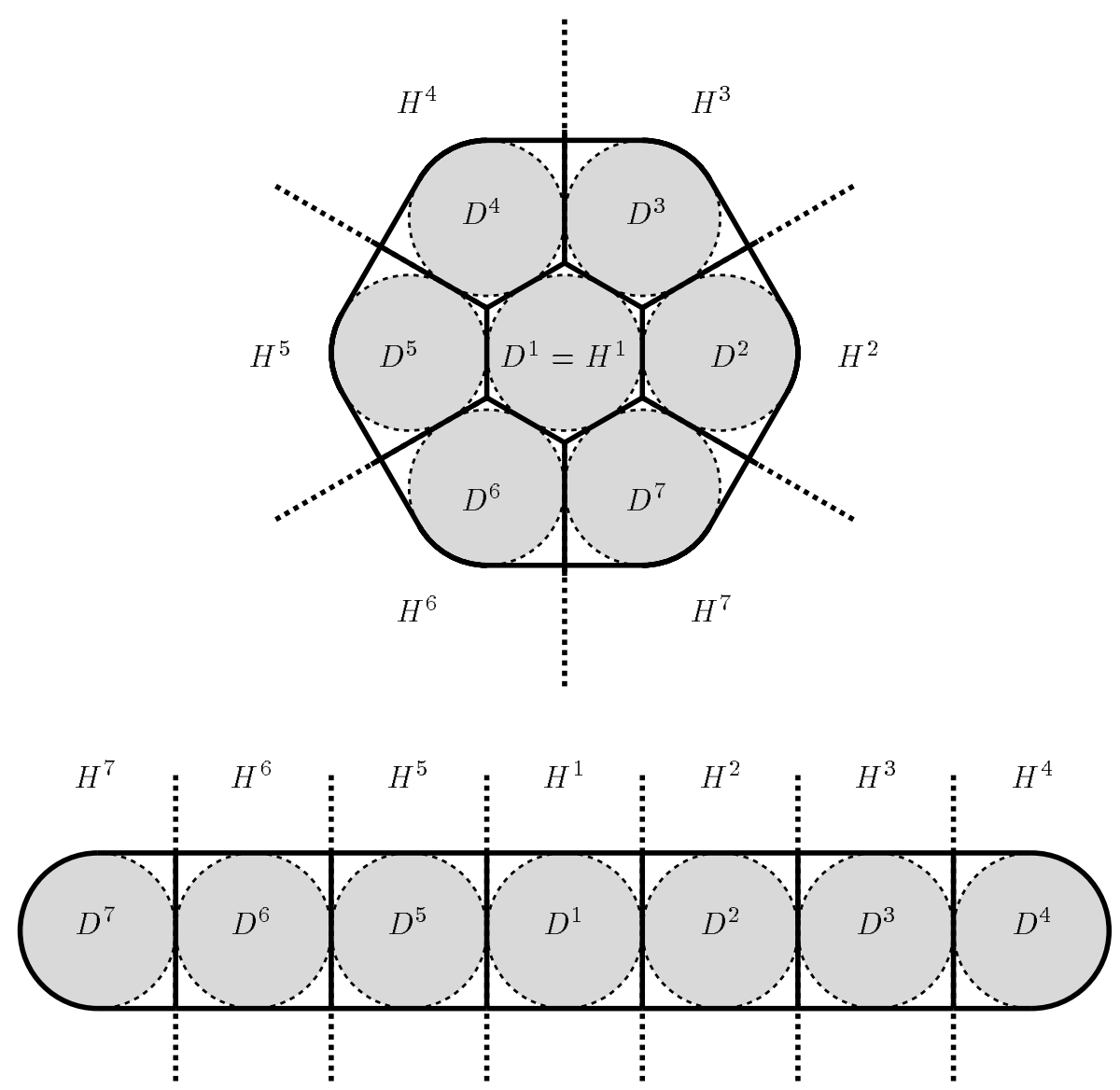

Figure 3.1.1.

where $\arccos (\cdot)$ is chosen in $[0, \pi / 2]$. Let $y^{j_{1}}, y^{j_{2}}$ be defined by $\arccos \left(\left|\left\langle y^{j_{1}}, y^{j_{2}}\right\rangle\right|\right)=\left\{\begin{array}{l}\phi: \text { if } \phi \geq \pi / 3 \text { or }\left\langle y^{k}, y^{l}\right\rangle \geq 0 \text { for } 1 \leq k, l \leq n-1, \\ \max _{1 \leq k, l \leq n-1}\left\{\arccos \left(\left|\left\langle y^{k}, y^{l}\right\rangle\right|\right):\left\langle y^{k}, y^{l}\right\rangle \leq 0\right\}: \text { otherwise. }\end{array}\right.$

Further let $L=\operatorname{lin}\left\{y^{j_{1}}, y^{j_{2}}\right\}$ and $U(\phi)=\operatorname{conv}\left\{0,2 y^{j_{1}}, 2 y^{j_{2}}\right\} \cap B^{d}$.

After renumbering, if necessary, we may assume $y^{1}=y^{j_{1}}$ and $y^{2}=y^{j_{2}}$. Such a pair $y^{1}, y^{2}$ may not be uniquely determined, but in any case the definition of $\phi$ and of $y^{1}, y^{2}$ gives us the information:

$$
\begin{aligned}
\left|\left\langle y^{k}, y^{l}\right\rangle\right| & \geq \cos (\phi), \quad 1 \leq k, l \leq n-1, \text { and } \\
\left|\left\langle y^{1}, y^{2}\right\rangle\right| & =\cos (\phi), \quad \text { if } \phi \geq \pi / 3 \text { or }\left\langle y^{k}, y^{l}\right\rangle \geq 0,1 \leq k, l \leq n-1, \\
\left\langle y^{1}, y^{2}\right\rangle & \in[-\cos (\phi / 2),-\cos (\phi)], \quad \text { otherwise. }
\end{aligned}
$$


For the lower bound in the last statement we have only to consider the case that there exists a pair $y^{m_{1}}, y^{m_{2}}$ with $\left\langle y^{m_{1}}, y^{m_{2}}\right\rangle=\cos (\phi)$. But, since $\phi<\pi / 3$, this implies $\left\langle y^{m_{1}}, y^{j}\right\rangle \geq-\cos (\phi / 2)$ or $\left\langle y^{m_{2}}, y^{j}\right\rangle \geq-\cos (\phi / 2)$ for $1 \leq j \leq n$ and hence $\left\langle y^{1}, y^{2}\right\rangle \geq-\cos (\phi / 2)$.

We see that a small angle $\phi$ indicates that in a neighborhood of 0 the arrangement is like the middle of a sausage arrangement for $\left\langle y^{1}, y^{2}\right\rangle<0$ or like the end of a sausage arrangement for $\left\langle y^{1}, y^{2}\right\rangle>0$, whereas a large $\phi$ means that the configuration $C$ is not sausage like.

Observe that $U(\phi)+\rho B^{d} \subset \operatorname{conv}(C)+\rho B^{d}$ and as $C \in \mathcal{P}\left(B^{d}, n\right)$ we have $B^{d} \subset H$ which implies $U(\phi) \subset H \cap \operatorname{conv}(C)$. Now we distinguish several parts of $D_{\rho}$ according to their position relative to $U(\phi)$. To this end we use the nearest point map (cf. [MS71]): For a convex body $K \in \mathcal{K}^{d}$ the nearest point map $p: E^{d} \rightarrow E^{d}$ with respect to $K$ is given by

$$
p(x)=y \in K \text { with }|x-y|=\min \{|x-z|: z \in K\} .
$$

Using the nearest point map with respect to $U(\phi)$ we define:

\section{Definition 3.1.2}

$$
\begin{aligned}
& D_{\rho}^{1}=\operatorname{cl}\left\{x \in D_{\rho}: p(x) \in \operatorname{relint}(U(\phi))\right\}, \\
& D_{\rho}^{2}=\operatorname{cl}\left\{x \in D_{\rho}: p(x) \in \operatorname{relint}\left(\operatorname{conv}\left\{0, y^{1}\right\}\right) \cup \operatorname{relint}\left(\operatorname{conv}\left\{0, y^{2}\right\}\right)\right\}, \\
& D_{\rho}^{3}=\operatorname{cl}\left\{x \in D_{\rho}: p(x)=0\right\}, \\
& D_{\rho}^{4}=\operatorname{cl}\left\{x \in D_{\rho}: p(x) \in \operatorname{relint}\left(\operatorname{conv}\left\{2 y^{1}, 2 y^{2}\right\}\right)\right\},
\end{aligned}
$$

where $\operatorname{cl}(S)$ (or $\operatorname{cl} S)$ denotes the closure of a set $S$ and $\operatorname{relint}(S)$ (or $\operatorname{relint} S$ ) denotes the interior of a set $S$ with respect to its affine hull. The sets $D_{\rho}^{i}$, $i=2,3,4$, may be considered as the sausage parts of $D_{\rho}$ and $D_{\rho}^{1}$ is the additional non-sausage part. Clearly

$$
V\left(D_{\rho}\right) \geq \sum_{i=1}^{4} V\left(D_{\rho}^{i}\right)
$$

The proof of (3.1.4) depends on various estimates of $V\left(D_{\rho}^{i}\right)$. These estimates are prepared by the following two lemmas.

Lemma 3.1.1 Let $w \in H \cap S^{d-1}, v \in w^{\perp} \cap S^{d-1}, \mu, \epsilon>0$ with $(\mu+\epsilon) v \in H$. Then

$$
c_{1}(\mu, \epsilon) \cdot \operatorname{conv}\{0, w\}+\mu v \subset H,
$$

with $c_{1}(\mu, \epsilon)=\epsilon / \sqrt{(\mu+\epsilon)^{2}-1}$ if $\mu \geq 1 /(\mu+\epsilon)$, else $c_{1}(\mu, \epsilon)=\sqrt{1-\mu^{2}}$.

Proof. The assertion follows with some elementary calculation from $B^{d} \subset H$ and the convexity of $H$ (see figure 3.1.2). 


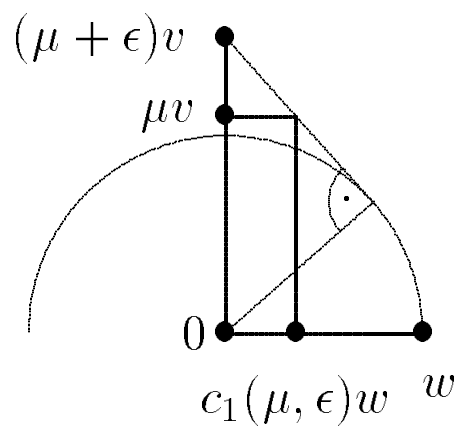

Figure 3.1.2.

Lemma 3.1.2 $V(U(\phi)) \geq \phi / 2$.

Proof. Let $\gamma=\left\langle y^{1}, y^{2}\right\rangle, \delta=\arccos (|\gamma|)$ and cone $\left\{y^{1}, y^{2}\right\}$ be the positive hull of $y^{1}, y^{2}$. First, suppose $\gamma \geq-1 / 2$. Then cone $\left\{y^{1}, y^{2}\right\} \cap B^{d} \subset U(\phi)$ and thus

$$
V(U(\phi)) \geq \delta / 2
$$

Next, assume $\gamma<-1 / 2$ and let $M=\left(\operatorname{cone}\left\{y^{1}, y^{2}\right\} \cap B^{d}\right) \backslash U(\phi)$ (see figure 3.1.3). Obviously, we have $V(U(\phi))=V\left(\operatorname{cone}\left\{y^{1}, y^{2}\right\} \cap B^{d}\right)-V(M)$ and by elementary calculation we get

$$
V(U(\phi))=\frac{\pi-\delta}{2}-\left(\arccos (2 \sin (\delta / 2))-2 \sin (\delta / 2) \sqrt{1-(2 \sin (\delta / 2))^{2}}\right) .
$$

On account of $\arcsin (x)=\pi / 2-\arccos (x)$ substituting $x=2 \sin (\delta / 2)$ in the right hand side yields $V(U(\phi))-\delta \geq \min \{f(x): x \in[0,1]\}$ with $f(x)=\arcsin (x)-$ $3 \arcsin (x / 2)+x \sqrt{1-x^{2}}$. Now, $f(0)=f(1)=0$, and for the second derivative

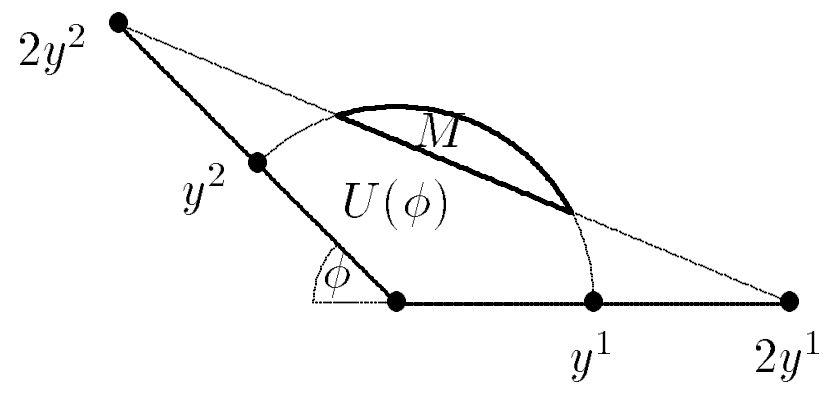

Figure 3.1.3. 
$f^{\prime \prime}(x)$ we have $f^{\prime \prime}(x) \leq 0$ for $x \in[0,1]$. Hence $f(x) \geq 0$ for $x \in[0,1]$ and

$$
V(U(\phi)) \geq \delta \text {. }
$$

If $\left\langle y^{1}, y^{2}\right\rangle \geq-1 / 2$ we have $\delta=\phi$ and in the case $\left\langle y^{1}, y^{2}\right\rangle<-1 / 2$ we have $\delta \geq \phi / 2$ (cf. (3.1.5)). Thus the assertion follows by (3.1.6) and (3.1.7).

Now, we start with the estimates for the sets $D_{\rho}^{i}$.

Lemma 3.1.3 Let $\sin (\phi) \leq 1 / \rho$. Then

$$
V\left(D_{\rho}^{1}\right) \geq \frac{\phi}{2} \cdot c_{1}(\rho, 1 / \sin (\phi)-\rho)^{2} \rho^{d-2} \kappa_{d-2} .
$$

Proof. By the definition of $\phi$ we have $\left|\left\langle y^{j}, y^{i}\right\rangle\right| \geq \cos (\phi)$ for $1 \leq j \leq n-1$, $i=1,2$. This implies $\left\langle y^{j}, v_{y^{i}}\right\rangle \leq \sin (\phi)$ for all $v_{y^{i}} \in\left(y^{i}\right)^{\perp} \cap B^{d}, i=1,2$. Hence by the definition of $H$ :

$$
(1 / \sin (\phi)) \cdot\left(\left(y^{i}\right)^{\perp} \cap B^{d}\right) \subset H, \quad i=1,2 .
$$

Thus $(1 / \sin (\phi))\left(L^{\perp} \cap B^{d}\right) \subset H$ and by lemma 3.1 .1 we get $c_{1}(\rho, 1 / \sin (\phi)-\rho)$. $U(\phi)+\rho\left(B^{d} \cap L^{\perp}\right) \subset D_{\rho}^{1}$. Now the lemma follows by the volume estimate given in lemma 3.1.2.

Lemma 3.1.4 Let $\sin (\phi) \leq 1 / \rho$. Then

$$
V\left(D_{\rho}^{2}\right) \geq c_{1}(\rho, 1 / \sin (\phi)-\rho) \rho^{d-1} \kappa_{d-1} .
$$

Proof. From (3.1.8) and lemma 3.1.1 follows

$$
c_{1}(\rho, 1 / \sin (\phi)-\rho) \cdot \operatorname{conv}\left\{0, y^{i}\right\}+\rho\left(\left(y^{i}\right)^{\perp} \cap B^{d}\right) \subset D_{\rho} .
$$

Let $a^{i} \in L$ be the outward normal unit vector of $\operatorname{conv}\left\{0, y^{i}\right\}$ with respect to $\operatorname{conv}\left\{0, y^{1}, y^{2}\right\}, i=1,2$. Then $\left\{x \in E^{d}:\left\langle a^{i}, x\right\rangle \geq 0\right\} \cap D_{\rho} \subset D_{\rho}^{2}$ and by (3.1.9) we get the assertion.

Lemma 3.1.5 Let $\sin (\phi) \leq 1 / \rho, \phi<\pi / 3$ and $\left\langle y^{1}, y^{2}\right\rangle>0$. Then

$$
V\left(D_{\rho}^{3}\right) \geq \frac{1-\phi / \pi}{2} \rho^{d} \kappa_{d}
$$

Proof. Let $F \subset L$ be the set of all outward unit normal vectors of supporting lines at 0 with respect to $\operatorname{conv}\left\{0, y^{1}, y^{2}\right\}$. By the definition of $y^{1}, y^{2}$ we have $\left\langle y^{i}, y^{k}\right\rangle \geq \cos (\phi)$ for $1 \leq k \leq n-1, i=1,2$, and thus $\left\langle y^{k}, a\right\rangle \leq 0,1 \leq k \leq n-1$, for all $a \in F$. As $\rho v \in D_{\rho}$ for $v \in L^{\perp} \cap B^{d}$ (cf. (3.1.8)) we get $\left(F+L^{\perp}\right) \cap \rho B^{d} \subset D_{\rho}^{3}$. Since $V(F)=(1-\phi / \pi) / 2$ we obtain the required estimate. 
Lemma 3.1.6 Let $\tan (\phi) \leq 1 / \rho, \phi<\pi / 3$ and $\left\langle y^{1}, y^{2}\right\rangle<0$. Then

$$
V\left(D_{\rho}^{4}\right) \geq \frac{\cos (\phi)-\rho \sin (\phi)}{\cos (\phi / 2)} \cdot \rho^{d-1} \kappa_{d-1}
$$

Proof. Let $w=\left(y^{1}-y^{2}\right) /\left|y^{1}-y^{2}\right|$. Since $\phi<\pi / 3$ we have $\left\langle y^{j}, y^{1}\right\rangle \geq \cos (\phi) \Leftrightarrow$ $\left\langle y^{j}, y^{2}\right\rangle \leq-\cos (\phi), 1 \leq j \leq n-1$. It follows $\left|\left\langle y^{j}, w\right\rangle\right| \geq \cos (\phi)$ which implies $\left\langle y^{j}, v\right\rangle \leq \sin (\phi)$ for all $v \in w^{\perp} \cap B^{d}$. Hence for $v \in w^{\perp} \cap B^{d}$ and $\lambda \in[0,1]$ we obtain

$$
\begin{aligned}
& \left\langle\lambda 2 y^{1}+(1-\lambda) 2 y^{2}+\rho v, y^{j}\right\rangle \leq \\
& \left\{\begin{array}{l}
\lambda(2 \cos (\phi)+2)-2 \cos (\phi)+\rho \sin (\phi):\left\langle y^{j}, y^{1}\right\rangle \geq \cos (\phi), \\
-\lambda(2 \cos (\phi)+2)+2+\rho \sin (\phi), \quad:\left\langle y^{j}, y^{1}\right\rangle \leq-\cos (\phi) .
\end{array}\right.
\end{aligned}
$$

This shows

$$
\lambda 2 y^{1}+(1-\lambda) 2 y^{2}+\rho\left(w^{\perp} \cap B^{d}\right) \subset H, \quad \text { for } \lambda \in\left[c_{2}(\phi, \rho), 1-c_{2}(\phi, \rho)\right],
$$

with $c_{2}(\phi, \rho)=(1+\rho \sin (\phi)) /(2+2 \cos (\phi))$. Observe, by assumption the given interval is nonempty and $\lambda 2 y^{1}+(1-\lambda) 2 y^{2} \in U(\phi)$. Thus

$$
\lambda 2 y^{1}+(1-\lambda) 2 y^{2}+\rho\left(w^{\perp} \cap B^{d}\right) \subset D_{\rho}, \lambda \in\left[c_{2}(\phi, \rho), 1-c_{2}(\phi, \rho)\right] .
$$

Let $u \in L$ be the outward unit normal vector of $\operatorname{conv}\left\{2 y^{1}, 2 y^{2}\right\}$ with respect to $\operatorname{conv}\left\{0,2 y^{1}, 2 y^{2}\right\}$ and let $v^{\prime} \in\left\{x \in E^{d}: x \in\left(w^{\perp} \cap B^{d}\right),\langle u, x\rangle \geq 0\right\}$. We have $\left(\operatorname{conv}\left\{2 y^{1}, 2 y^{2}\right\}+\rho v^{\prime}\right) \cap D_{\rho} \subset D_{\rho}^{4}$ and therefore, we obtain

$$
V\left(D_{\rho}^{4}\right) \geq\left(1-2 c_{2}(\phi, \rho)\right) \cdot\left|2 y^{1}-2 y^{2}\right| \rho^{d-1} \frac{\kappa_{d-1}}{2} .
$$

In the last four lemmas we made the assumption that $\phi$ is small. So in order to prove the theorem we need also an estimate for $V\left(D_{\rho}\right)$ if $\phi$ is large. In this case the angle gives us no valuable information about the position of the facets of $H$ but instead of it we know that $V(U(\phi))$ is not too small. Furthermore, in the lemmas we exploited the fact that the $(d-2)$-ball $\rho\left(L^{\perp} \cap B^{d}\right)$ is contained in $H$. Obviously for $\phi=\pi / 2$ and $\rho>1$ we cannot guarantee that this still holds. In the next lemma, which is the key for the whole proof, we show that a least a sufficiently large part of the $(d-2)$-ball belongs to $H$.

Lemma 3.1.7 Let $K(\rho)=\left\{z \in L^{\perp} \cap S^{d-1}: \rho z \in H\right\}$ and $d_{0}(\rho)=\max \{4$, $\left.\left(4-\rho^{2}\right) /\left(2-\rho^{2}\right)\right\}$. There is a constant $c(\rho)$ only depending on $\rho$ such that for all $d \geq d_{0}(\rho)$ :

$$
V_{*}(K(\rho)) \geq \frac{(d-2) \kappa_{d-2}}{1+c(\rho)}
$$

where $V_{*}(\cdot)$ denotes the spherical volume. 
The proof of lemma 3.1.7 is quite complex and hence we first state two preparing lemmas.

Lemma 3.1.8 Let $S \subset E^{d}$ be a d-simplex, $F_{k}$ be a $k$-face of $S, k \leq d-1$, and let $\bar{F}_{k}$ be the $(d-k-1)$-face of $S$ with $F_{k} \cap \bar{F}_{k}=\emptyset$. For a measurable subset $G \subset S$ and a continuous function $f$ on $S$ we have

$$
\begin{aligned}
\int_{G} f d x= & \frac{d !}{k !(d-1-k) !} \frac{V(S)}{V\left(F_{k}\right) V\left(\bar{F}_{k}\right)} . \\
& \int_{F_{k}} \int_{\bar{F}_{k}} \int_{\mu \bar{x}+(1-\mu) x \in G} f(\mu \bar{x}+(1-\mu) x) \mu^{d-1-k}(1-\mu)^{k} d \mu d \bar{x} d x .
\end{aligned}
$$

Remark: The notation $\int d x$ means integration in a space of appropriate dimension.

Proof. To compute the integral let $S=\operatorname{conv}\left\{a^{0}, \ldots, a^{d}\right\}, F_{k}=\operatorname{conv}\left\{a^{0}, \ldots, a^{k}\right\}$, $\bar{F}_{k}=\operatorname{conv}\left\{a^{k+1}, \ldots, a^{d}\right\}$. Further let $A, A_{k}, \bar{A}_{k}$ denote the matrices $A=\left(a^{1}-\right.$ $\left.a^{0}, \ldots, a^{d}-a^{0}\right), A_{k}=\left(a^{1}-a^{0}, \ldots, a^{k}-a^{0}\right), \bar{A}_{k}=\left(a^{k+2}-a^{k+1}, \ldots, a^{d}-a^{k+1}\right)$. Finally we denote by $T^{d}$ the standard simplex $T^{d}=\left\{\left(x_{1}, \ldots, x_{d}\right)^{T} \in E^{d}: x_{i} \geq\right.$ $\left.0, \sum_{i=1}^{d} x_{i} \leq 1\right\}$.

Now we apply a number of transformations. First let $\psi_{1}: E^{d} \rightarrow E^{d}$ given by $\psi_{1}(x)=A x+a^{0}$. Then $T^{d}=\psi_{1}^{-1}(S)$ and thus

$$
\int_{G} f(x) d x=d ! V(S) \int_{\psi_{1}^{-1}(G)} f\left(\psi_{1}(x)\right) d x .
$$

Next we consider $\psi_{2}: E^{k} \times E^{1} \times E^{d-1-k} \rightarrow E^{d}$ with $\psi_{2}\left(\mu_{1}, \ldots, \mu_{k}, \mu, \mu_{k+2}, \ldots, \mu_{d}\right)$ $=(1-\mu) \cdot\left(\mu_{1}, \ldots, \mu_{k}, 0, \ldots, 0\right)^{T}+\mu \cdot\left(0, \ldots, 0,1-\sum_{i=k+2}^{d} \mu_{i}, \mu_{k+2}, \ldots, \mu_{d}\right)^{T} \cdot \psi_{2}$ is a bijection from the interior of $T^{k} \times T^{1} \times T^{d-1-k}$ onto the interior of $T^{d}$. By a straightforward calculation we find for the determinant of the Jacobian $D \psi_{2}$ of $\psi_{2} \operatorname{det}\left(D \psi_{2}\right)=\mu^{d-1-k}(1-\mu)^{k}$. Hence with $\mu^{\prime}=\left(\mu_{1}, \ldots, \mu_{k}\right), \bar{\mu}=\left(\mu_{k+2}, \ldots, \mu_{d}\right)$ and (3.1.10)

$$
\begin{aligned}
& \int_{G} f(x) d x=d ! V(S) \times \\
& \quad \int_{T^{k}} \int_{T^{d-1-k}} \int_{\psi_{2}\left(\mu^{\prime}, \mu, \bar{\mu}\right) \in \psi_{1}^{-1}(G)} f\left(\psi_{1}\left(\psi_{2}\left(\mu^{\prime}, \mu, \bar{\mu}\right)\right)\right) \mu^{d-1-k}(1-\mu)^{k} d \mu d \bar{\mu} d \mu^{\prime} .
\end{aligned}
$$

As $\psi_{1}\left(\psi_{2}\left(\mu^{\prime}, \mu, \bar{\mu}\right)\right)=(1-\mu)\left(A_{k} \mu^{\prime}+a^{0}\right)+\mu\left(\bar{A}_{k} \bar{\mu}+a^{k+1}\right), F_{k}=\left\{A_{k} \mu^{\prime}+a^{0}: \mu^{\prime} \in T^{k}\right\}$ and $\bar{F}_{k}=\left\{\bar{A}_{k} \bar{\mu}+a^{k+1}: \bar{\mu} \in T^{d-1-k}\right\}$ we obtain the formula.

Moreover, we need that the quotient of two certain integrals is bounded:

Lemma 3.1.9 Let $k, \bar{k} \in \mathbb{N}$ with $\bar{k} \geq k+1$ and let $\alpha, \beta, \gamma \in \mathbb{R}$ with $\gamma>\beta>0$, $\alpha>0$. Then for $a, b, c \in \mathbb{R}, d \in \mathbb{N}$ with $b, c \geq 0, b<c, a \geq \alpha, a^{2}+c^{2} \geq \gamma$, $a^{2}+b^{2} \leq \beta, d \geq \bar{k}$ the quotient 


$$
\frac{\int_{0}^{\mu_{0}}\left(\sqrt{a^{2}+(\mu c+(1-\mu) b)^{2}}\right)^{-(d+1)} \mu^{d-1-k}(1-\mu)^{k} d \mu}{\int_{\mu_{0}}^{1}\left(\sqrt{a^{2}+(\mu c+(1-\mu) b)^{2}}\right)^{-(d+1)} \mu^{d-1-k}(1-\mu)^{k} d \mu}
$$

where $\mu_{0} \in[0,1]$ is determined by $a^{2}+\left(\mu_{0} c+\left(1-\mu_{0}\right) b\right)^{2}=\beta$, is maximal for $a=\alpha, b=0, a^{2}+c^{2}=\gamma$ and $d=\bar{k}$.

Proof. First we show that the quotient is monotonely decreasing in $a, b, c, d$. To do this we set

$$
f(a, b, c, d, \mu)=\left(\sqrt{a^{2}+(\mu c+(1-\mu) b)^{2}}\right)^{-(d+1)} \mu^{d-1-k}(1-\mu)^{k} .
$$

For $d$ we have by the mean value theorem of integral calculus:

$$
\frac{\int_{0}^{\mu_{0}} f(a, b, c, d+1, \mu) d \mu}{\int_{\mu_{0}}^{1} f(a, b, c, d+1, \mu) d \mu}=\frac{\mu_{1} / \sqrt{a^{2}+\left(\mu_{1} c+\left(1-\mu_{1}\right) b\right)^{2}}}{\mu_{2} / \sqrt{a^{2}+\left(\mu_{2} c+\left(1-\mu_{2}\right) b\right)^{2}}} \cdot \frac{\int_{0}^{\mu_{0}} f(a, b, c, d, \mu) d \mu}{\int_{\mu_{0}}^{1} f(a, b, c, d, \mu) d \mu}
$$

for suitable $0 \leq \mu_{1} \leq \mu_{0} \leq \mu_{2} \leq 1$. Since the function $\mu / \sqrt{a^{2}+(\mu c+(1-\mu) b)^{2}}$ is monotonely increasing in $\mu$ we get that (3.1.11) is monotonely decreasing in $d$.

Now let $c^{\prime}>c$ and let $\mu_{0}^{\prime} \in[0,1]$ be given by $a^{2}+\left(\mu_{0}^{\prime} c^{\prime}+\left(1-\mu_{0}^{\prime}\right) b\right)^{2}=\beta$. We apply the linear transformation $\mu=(c-b) /\left(c^{\prime}-b\right) \mu^{\prime}$ and obtain

$$
\frac{\int_{0}^{\mu_{0}^{\prime}} f\left(a, b, c^{\prime}, d, \mu\right) d \mu}{\int_{\mu_{0}^{\prime}}^{1} f\left(a, b, c^{\prime}, d, \mu\right) d \mu}=\frac{\left(\frac{c-b}{c^{\prime}-b}\right)^{d-k} \int_{0}^{\mu_{0}} f\left(a, b, c, d, \mu^{\prime}\right)\left(\frac{1-(c-b) /\left(c^{\prime}-b\right) \mu^{\prime}}{1-\mu^{\prime}}\right)^{k} d \mu^{\prime}}{\left(\frac{c-b}{c^{\prime}-b}\right)^{d-k} \int_{\mu_{0}}^{\left(c^{\prime}-b\right) /(c-b)} f\left(a, b, c, d, \mu^{\prime}\right)\left(\frac{1-(c-b) /\left(c^{\prime}-b\right) \mu^{\prime}}{1-\mu^{\prime}}\right)^{k} d \mu^{\prime}} .
$$

As $\left(c^{\prime}-b\right) /(c-b)>1$ and $\left(1-(c-b) /\left(c^{\prime}-b\right) \mu^{\prime}\right) /\left(1-\mu^{\prime}\right)$ is monotonely increasing for $0 \leq \mu^{\prime} \leq 1$ we find again from the mean value theorem of integral calculus:

$$
\frac{\int_{0}^{\mu_{0}^{\prime}} f\left(a, b, c^{\prime}, d, \mu\right) d \mu}{\int_{\mu_{0}^{\prime}}^{1} f\left(a, b, c^{\prime}, d, \mu\right) d \mu} \leq \frac{\int_{0}^{\mu_{0}} f\left(a, b, c, d, \mu^{\prime}\right) d \mu^{\prime}}{\int_{\mu_{0}}^{1} f\left(a, b, c, d, \mu^{\prime}\right) d \mu^{\prime}} .
$$

Quite analogously we find that (3.1.11) is monotonely decreasing in $b$.

Finally let $a^{\prime}>a$. For the corresponding value $\mu_{0}^{\prime}$ we have $\mu_{0}^{\prime}<\mu_{0}$ and further we may write $a^{\prime 2}+(\mu c+(1-\mu) b)^{2}=g(\mu)\left(a^{2}+(\mu c+(1-\mu) b)^{2}\right)$ for an appropriate function $g$. We easily check that $g$ is monotonely decreasing in $\mu$ for $\mu \geq 0$. Thus we find as before:

$$
\frac{\int_{0}^{\mu_{0}^{\prime}} f\left(a^{\prime}, b, c, d, \mu\right) d \mu}{\int_{\mu_{0}^{\prime}}^{1} f\left(a^{\prime}, b, c, d, \mu\right) d \mu}<\frac{\int_{0}^{\mu_{0}} f\left(a^{\prime}, b, c, d, \mu\right) d \mu}{\int_{\mu_{0}}^{1} f\left(a^{\prime}, b, c, d, \mu\right) d \mu} \leq \frac{\int_{0}^{\mu_{0}} f(a, b, c, d, \mu) d \mu}{\int_{\mu_{0}}^{1} f(a, b, c, d, \mu) d \mu} .
$$

Hence we may assume $d=\bar{k}, b=0$ and $c^{2}=\gamma-a^{2}$. In this case we have $\mu_{0}=\sqrt{\left(\beta-a^{2}\right) /\left(\gamma-a^{2}\right)}$ and $\mu_{0}$ is monotonely decreasing in $a$. Now let $a^{\prime}>a$, 
$c^{\prime}=\gamma-\left(a^{\prime}\right)^{2}$, and $\mu_{0}^{\prime}$ the value of $\mu_{0}$ with respect to $a^{\prime}$ and $c^{\prime}$. The function $g(\mu)$ defined by $a^{\prime 2}+\left(\mu c^{\prime}\right)^{2}=g(\mu)\left(a^{2}+(\mu c)^{2}\right)$ is monotonely decreasing in $\mu$ and as above we find

$$
\frac{\int_{0}^{\mu_{0}^{\prime}} f\left(a^{\prime}, 0, c^{\prime}, d, \mu\right) d \mu}{\int_{\mu_{0}^{\prime}}^{1} f\left(a^{\prime}, 0, c^{\prime}, d, \mu\right) d \mu}<\frac{\int_{0}^{\mu_{0}} f\left(a^{\prime}, 0, c^{\prime}, d, \mu\right) d \mu}{\int_{\mu_{0}}^{1} f\left(a^{\prime}, 0, c^{\prime}, d, \mu\right) d \mu} \leq \frac{\int_{0}^{\mu_{0}} f(a, 0, c, d, \mu) d \mu}{\int_{\mu_{0}}^{1} f(a, 0, c, d, \mu) d \mu} .
$$

Hence the integral in (3.1.11) is increased if $a$ is decreased and $a^{2}+c^{2}$ kept constant. Thus the lemma is proved.

Now we come to the proof of lemma 3.1.7:

Proof of lemma 3.1.7. Let $\rho<\sqrt{2}, d \geq d_{0}(\rho)$ and $M(\rho)=\left\{z \in L^{\perp} \cap S^{d-1}\right.$ : $\rho z \notin H\}$. Since

$$
V_{*}(K(\rho))=\frac{V_{*}(K(\rho))+V_{*}(M(\rho))}{1+V_{*}(M(\rho)) / V_{*}(K(\rho))}=\frac{(d-2) \kappa_{d-2}}{1+V_{*}(M(\rho)) / V_{*}(K(\rho))}
$$

it suffices to show that there exists a constant $c(\rho)$ only depending on $\rho$ such that for $d \geq d_{0}(\rho)$ :

$$
V_{*}(M(\rho)) / V_{*}(K(\rho)) \leq c(\rho) .
$$

To this end we consider the bounded polyhedron $H \cap W_{\sqrt{2}}^{d}$ where $W_{\sqrt{2}}^{d}$ denotes the $d$-cube with center 0 and edge length $2 \sqrt{2}$. Observe, that

$$
\begin{aligned}
M(\rho) & =\left\{z \in L^{\perp} \cap S^{d-1}: \rho z \notin H \cap W_{\sqrt{2}}^{d}\right\}, \\
K(\rho) & =\left\{z \in L^{\perp} \cap S^{d-1}: \rho z \in H \cap W_{\sqrt{2}}^{d}\right\} .
\end{aligned}
$$

We use a technique due to Rogers' [Rog64] which gives by an inductive construction a dissection of the intersection $\left(H \cap W_{\sqrt{2}}^{d}\right) \cap L^{\perp}$ into simplices $S$ of the form $S=\operatorname{conv}\left\{c^{0}, \ldots, c^{d-2}\right\}$ such that each $c^{i}$ is contained in a $(d-2-i)$-face $F$ of $\left(H \cap W_{\sqrt{2}}^{d}\right) \cap L^{\perp}$ which contains conv $\left\{c^{i}, \ldots, c^{d-2}\right\}$ and $c^{i}$ is the nearest point of $F$ to 0 .

Let $c^{0}=0$ and assume we have chosen a sequence of points $\left\{c^{0}, \ldots, c^{i}\right\}$ such that $c^{j}$ lies in a $(d-2-j)$-face $F_{d-2-j}$ of $\left(H \cap W_{\sqrt{2}}^{d}\right) \cap L^{\perp}$ and is the nearest point of the face to the origin, $0 \leq j \leq i$. Let $F_{d-3-i}^{1}, \ldots, F_{d-3-i}^{k}$ be the facets of $F_{d-2-i}$ which do not contain $c^{i}$. Then for $1 \leq l \leq k$ we define the point $c^{i+1, l}$ to be the point in $F_{d-3-i}^{l}$ nearest to 0 . So we obtain sequences $\left\{c^{0}, \ldots, c^{i}, c^{i+1, l}\right\}$, $1 \leq l \leq k$, and at the end of the construction $(i=d-2)$ we obtain a dissection of the desired form.

If $c^{i}$ belongs to a face of $W_{\sqrt{2}}^{d}$ then we clearly have $\left|c^{i}\right| \geq \sqrt{2}$. Otherwise, as $c^{i}$ lies in a $(d-2-i)$-face it belongs to a $(d-i)$-face of $H$ and by a result of Rogers [Rog64] we have $\left|c^{i}\right| \geq \sqrt{2} \sqrt{i /(i+1)}$. Thus we get for $0 \leq i \leq d-2$ :

$$
\left|c^{i}\right| \geq \sqrt{2} \sqrt{i /(i+1)} .
$$


Let $S=\operatorname{conv}\left\{c^{0}, \ldots, c^{d-2}\right\}$ be an arbitrary but fixed simplex of this dissection, $\mathcal{C}$ be the cone generated by $c^{1}, \ldots, c^{d-2}$ and let

$$
M=\left\{z \in\left(L^{\perp} \cap S^{d-1}\right) \cap \mathcal{C}: \rho z \notin S\right\}, \quad K=\left\{z \in\left(L^{\perp} \cap S^{d-1}\right) \cap \mathcal{C}: \rho z \in S\right\} .
$$

To show that $V_{*}(M(\rho)) / V_{*}(K(\rho))$ is bounded from above it is clearly sufficient to show that $V_{*}(M) / V_{*}(K)$ is bounded from above. In order to evaluate these spherical volumes we apply several transformations. First we replace the sets $M, K$ by the associated parts $M_{B}, K_{B}$ of the $d$-ball, i.e.

$M_{B}=\left\{z \in\left(L^{\perp} \cap B^{d}\right) \cap \mathcal{C}: \rho z /|z| \notin S\right\}, K_{B}=\left\{z \in\left(L^{\perp} \cap B^{d}\right) \cap \mathcal{C}: \rho z /|z| \in S\right\}$.

Clearly, we have $V_{*}(M) / V_{*}(K)=V\left(M_{B}\right) / V\left(K_{B}\right)$. In the next step we express $V\left(M_{B}\right), V\left(K_{B}\right)$ as integrals over the simplex $S$. To this end let $v \in L^{\perp} \cap S^{d-1}$ be the outward unit normal vector of the facet $F=\operatorname{conv}\left\{c^{1}, \ldots, c^{d-2}\right\}$ with respect to $S$ and let $\eta \in \mathbb{R}$ be the distance of the affine hull of $F$ to the origin. We consider the map $\psi: E^{d} \cap L^{\perp} \rightarrow E^{d} \cap L^{\perp}$ given by $\psi(x)=\frac{\langle v, x\rangle}{\eta|x|} x$. For the determinant of the Jacobian we find $\operatorname{det}(D \psi)=(\langle v, x\rangle /(\eta|x|))^{d-2}$ and with

$$
\begin{aligned}
M_{S} & =\psi^{-1}\left(M_{B}\right)=\{z \in S: \rho z /|z| \notin S\} \\
K_{S} & =\psi^{-1}\left(K_{B}\right)=\{z \in S: \rho z /|z| \in S\}
\end{aligned}
$$

we obtain

$$
V\left(M_{B}\right)=\int_{M_{S}}\left(\frac{\langle v, x\rangle}{\eta|x|}\right)^{d-2} d x, \quad V\left(K_{B}\right)=\int_{K_{S}}\left(\frac{\langle v, x\rangle}{\eta|x|}\right)^{d-2} d x .
$$

Now we use lemma 3.1 .8 with $k=0, F_{0}=0$ and $\bar{F}_{0}=F$. As $\langle v, x\rangle /(\eta|x|)$ is constant on rays emanating from 0 we get

$$
V\left(M_{B}\right)=\frac{\eta}{d-2} \int_{M_{F}}\left(\frac{1}{|x|}\right)^{d-2} d x, \quad V\left(K_{B}\right)=\frac{\eta}{d-2} \int_{K_{F}}\left(\frac{1}{|x|}\right)^{d-2} d x
$$

with $M_{F}=\{z \in F:|z| \leq \rho\}$ and $K_{F}=\{z \in F:|z| \geq \rho\}$.

Finally, by the definition of $d_{0}(\rho)$ we may choose a $k \in \mathbb{N}, d-4 \geq k$, with $\sqrt{2} \sqrt{(k+2) /(k+3)}>\rho$ (cf. (3.1.13)). We apply lemma 3.1 .8 to the $(d-3)$ simplex $F$ and the faces $F_{k}=\operatorname{conv}\left\{c^{1}, \ldots, c^{k+1}\right\}, \bar{F}_{k}=\operatorname{conv}\left\{c^{k+2}, \ldots, c^{d-2}\right\}$. Defining

$$
\xi\left(F, F_{k-1}\right)=\frac{\eta}{d-2} \frac{(d-3) !}{k !(d-4-k) !} \frac{V(F)}{V\left(F_{k}\right) V\left(\bar{F}_{k}\right)}
$$

we may write by (3.1.14):

$$
\begin{aligned}
V\left(M_{B}\right) & =\xi\left(F, F_{k}\right) \int_{F_{k}} \int_{\bar{F}_{k}} \int_{|\mu \bar{x}+(1-\mu) x| \leq \rho} \frac{\mu^{d-4-k}(1-\mu)^{k}}{|\mu \bar{x}+(1-\mu) x|^{d-2}} d \mu d \bar{x} d x \\
V\left(K_{B}\right) & =\xi\left(F, F_{k}\right) \int_{F_{k}} \int_{\bar{F}_{k}} \int_{|\mu \bar{x}+(1-\mu) x| \geq \rho} \frac{\mu^{d-4-k}(1-\mu)^{k}}{|\mu \bar{x}+(1-\mu) x|^{d-2}} d \mu d \bar{x} d x .
\end{aligned}
$$


Thus we have

$$
\frac{V_{*}(M)}{V_{*}(K)} \leq \max \left\{\frac{\int_{|\mu \bar{x}+(1-\mu) x| \leq \rho} \frac{\mu^{d-4-k}(1-\mu)^{k}}{|\mu \bar{x}+(1-\mu) x|^{d-2}} d \mu}{\int_{|\mu \bar{x}+(1-\mu) x| \geq \rho} \frac{\mu^{d-4-k}(1-\mu)^{k}}{|\mu \bar{x}+(1-\mu) x|^{d-2}} d \mu}: x \in F_{k}, \bar{x} \in \bar{F}_{k}\right\} .
$$

It remains to show

$$
\frac{\int_{|\mu \bar{x}+(1-\mu) x| \leq \rho}|\mu \bar{x}+(1-\mu) x|^{-(d-2)} \mu^{d-4-k}(1-\mu)^{k} d \mu}{\int_{|\mu \bar{x}+(1-\mu) x| \geq \rho}|\mu \bar{x}+(1-\mu) x|^{-(d-2)} \mu^{d-4-k}(1-\mu)^{k} d \mu} \leq \tilde{c}(\rho),
$$

for all $x \in F_{k}, \bar{x} \in \bar{F}_{k}$ and a suitable constant $\tilde{c}(\rho)$ only depending on $\rho$.

By construction of $S$ we have that $\mid \mu \bar{x}+(1-\mu) x) \mid$ is strictly increasing in $\mu$. Further, we have $|x| \geq 1$ and by the choice of $k$ :

$$
|\bar{x}| \geq \sqrt{2} \sqrt{\frac{k+2}{k+3}}>\rho
$$

So for $|x| \geq \rho$ there is nothing to prove. Else we find that the inequality (3.1.16) is of the form

$$
\frac{\int_{0}^{\mu_{0}}\left(\sqrt{a^{2}+(\mu c+(1-\mu) b)^{2}}\right)^{-(d-2)} \mu^{d-4-k}(1-\mu)^{k} d \mu}{\int_{\mu_{0}}^{1}\left(\sqrt{a^{2}+(\mu c+(1-\mu) b)^{2}}\right)^{-(d-2)} \mu^{d-4-k}(1-\mu)^{k} d \mu},
$$

where $a \geq \alpha=1$ denotes the distance of the line through $\bar{x}, x$ from $0, b$ is given by $a^{2}+b^{2}=|x|^{2}, c$ by $a^{2}+c^{2}=|\bar{x}|^{2}$ and $\mu_{0}$ is determined by $a^{2}+\left(\mu_{0} c+\left(1-\mu_{0}\right) b\right)^{2}=\rho^{2}$. But now (3.1.16) follows from lemma 3.1.9 with $\beta=\rho^{2}$ and $\gamma=2(k+2) /(k+3)$ and lemma 3.1.7 is proved.

By lemma 3.1.7 we can now give an estimate for the volume of $D_{\rho}^{1}$ for all $\phi>0$.

Lemma 3.1.10 Let $\phi>0, \epsilon>0$ with $\rho_{1}=\rho+\epsilon<\sqrt{2}$ and $d_{0}\left(\rho_{1}\right)=\max \{4,(4-$ $\left.\left.\rho_{1}^{2}\right) /\left(2-\rho_{1}^{2}\right)\right\}$. There is a constant $c\left(\rho_{1}\right) \in \mathbb{R}$ only depending on $\rho_{1}$ such that for $d \geq d_{0}\left(\rho_{1}\right)$ :

$$
V\left(D_{\rho}^{1}\right) \geq \frac{\phi}{2} c_{1}(\rho, \epsilon)^{2} \rho^{d-2} \cdot \frac{\kappa_{d-2}}{1+c\left(\rho_{1}\right)}
$$

Proof. From the definition of $D_{\rho}^{1}$ we have

$$
V\left(D_{\rho}^{1}\right) \geq \frac{\rho^{d-2}}{d-2} \int_{U(\phi)} \int_{\left\{z \in L^{\perp} \cap S^{d-1}: w+\rho z \in H\right\}} d v d w
$$


Instead of evaluating the inner integral for each point $w \in U(\phi)$, we only estimate the integral at the point 0 but for the larger value $\rho_{1}$. On account of lemma 3.1.1 and lemma 3.1.2 we obtain with the notation of lemma 3.1.7:

$$
V\left(D_{\rho}^{1}\right) \geq \frac{\phi}{2} c_{1}(\rho, \epsilon)^{2} \frac{\rho^{d-2}}{d-2} V_{*}\left(K\left(\rho_{1}\right)\right) \geq \frac{\phi}{2} c_{1}(\rho, \epsilon)^{2} \rho^{d-2} \frac{\kappa_{d-2}}{1+c\left(\rho_{1}\right)},
$$

for $d \geq d_{0}\left(\rho_{1}\right)$.

On account of the previous lemmas the proof of theorem 3.1.1 is an easy consequence of

$$
\lim _{d \rightarrow \infty} \kappa_{d-2} / \kappa_{d-1}=\infty .
$$

Proof of theorem 3.1.1. Let $\rho<\sqrt{2}$ and $\phi_{0}=\min \{\arctan (1 / \rho), \pi / 3\}$. We distinguish three cases depending on $\phi$ and the sign of $\left\langle y^{1}, y^{2}\right\rangle$. For simplification we use $V\left(D_{\rho}^{2}\right) \geq(1-\rho \sin (\phi)) \rho^{d-1} \kappa_{d-1}$ (cf. lemma 3.1.4).

I). $\phi<\phi_{0}$ and the assumptions of lemma 3.1.5 hold. Then we have by lemma 3.1.3, 3.1.4 and 3.1.5:

$$
\begin{aligned}
V\left(D_{\rho}\right) & \geq V\left(D_{\rho}^{1}\right)+V\left(D_{\rho}^{2}\right)+V\left(D_{\rho}^{3}\right) \\
& \geq \rho^{d-1} \kappa_{d-1}+\frac{\rho^{d} \kappa_{d}}{2}+\phi \rho^{d-2}\left(\frac{1}{2} \frac{\left(1-\rho \sin \left(\phi_{0}\right)\right)^{2}}{1-\sin ^{2}\left(\phi_{0}\right)} \kappa_{d-2}-\rho^{2} \kappa_{d-1}-\frac{\rho^{2} \kappa_{d}}{2 \pi}\right) \\
& =\rho^{d-1} \kappa_{d-1}+\frac{\rho^{d} \kappa_{d}}{2}+\phi \cdot f_{1}(\rho, d) .
\end{aligned}
$$

By (3.1.20) there exists a $d_{1}(\rho) \in \mathbb{N}$ with $f_{1}(\rho, d)>0$ for all $d \geq d_{1}(\rho)$.

II). $\phi<\phi_{0}$ and the assumptions of lemma 3.1.6 hold. Then we have by lemma 3.1.3, 3.1.4 and 3.1.6 and $\cos (\phi) \geq 1-\phi^{2} / 2$ :

$$
\begin{aligned}
V\left(D_{\rho}\right) & \geq V\left(D_{\rho}^{1}\right)+V\left(D_{\rho}^{2}\right)+V\left(D_{\rho}^{4}\right) \\
& \geq 2 \rho^{d-1} \kappa_{d-1}+\phi \rho^{d-2}\left(\frac{1}{2} \frac{\left(1-\rho \sin \left(\phi_{0}\right)\right)^{2}}{1-\sin ^{2}\left(\phi_{0}\right)} \kappa_{d-2}-2 \rho^{2} \kappa_{d-1}-\frac{\phi_{0}}{2} \rho \kappa_{d-1}\right) \\
& =2 \rho^{d-1} \kappa_{d-1}+\phi \cdot f_{2}(\rho, d) .
\end{aligned}
$$

Let $d_{2}(\rho) \in \mathbb{N}$ with $f_{2}(\rho, d)>0$ for all $d \geq d_{2}(\rho)$.

III). $\phi \geq \phi_{0}$. Choose an $\epsilon$ such that the assumption of lemma 3.1.10 holds. Then by lemma 3.1 .10 with $\rho_{1}=\rho+\epsilon$ and for $d \geq d_{0}\left(\rho_{1}\right)$ :

$$
\begin{aligned}
V\left(D_{\rho}\right) & \geq V\left(D_{\rho}^{1}\right) \geq \frac{\phi_{0}}{2} \cdot c_{1}(\rho, \epsilon)^{2} \rho^{d-2} \cdot \frac{\kappa_{d-2}}{1+c\left(\rho_{1}\right)} \\
& >2 \rho^{d-1} \kappa_{d-1}
\end{aligned}
$$

for all $d \geq d_{3}(\rho)$, say.

Finally let $d(\rho) \in \mathbb{N}$ given by $d(\rho)=\max \left\{d_{1}(\rho), d_{2}(\rho), d_{3}(\rho)\right\}$. Since the first case $(\phi<\pi / 3$ and all inner products are positive) can occur at most twice we have proved (3.1.4) and thus (3.1.1). Furthermore, it follows immediately from the proof that $S_{n}\left(B^{d}\right)$ is the only best possible packing set. 


\subsection{Finite packings of centrally symmetric bodies}

In this section we prove an extension of theorem 3.1.1 to arbitrary centrally symmetric convex bodies.

Theorem 3.2.1 There is a positive function $\psi(x, y)$ on $[1, \infty) \times \mathbb{N}$ with

$$
\lim _{y \rightarrow \infty} \psi(1, y)=\sqrt{2} \quad \text { and } \quad \psi\left(x_{1}, y\right)>\psi\left(x_{2}, y\right) \text { if } x_{1}<x_{2},
$$

such that for $K \in \mathcal{K}_{0}^{d}, d \geq 5$, with inradius $r(K)$ and circumradius $R(K)$ and for $\rho<\psi(R(K) / r(K), d)$ :

$$
V(\operatorname{conv}(C)+\rho K) \geq V\left(\operatorname{conv}\left(S_{n}(K)\right)+\rho K\right)
$$

for all $C \in \mathcal{P}(K, n), n \in \mathbb{N}$. Equality holds if and only if $C$ is a densest sausage configuration of $K$.

So, the theorem states for a centrally symmetric convex body of a given dimension a bound for the parameter $\rho$ such that a sausage arrangement becomes best possible with respect to the densities induced by the parameter $\rho$. In particular for the $d$-dimensional unit ball we obtain asymptotically the bound already given in corollary 3.1.1. Indeed, the proof of theorem 3.2.1 is based on an adjustment of the methods and techniques used in the proof of theorem 3.1.1 to centrally symmetric bodies. Unfortunately, the necessary changes are quite technical.

In the sequel we consider for a fixed dimension $d$ a $K \in \mathcal{K}_{0}^{d}$ with distance function $f: E^{d} \rightarrow \mathbb{R}$, inradius $r$ and circumradius $R$. As in the proof of theorem 3.1.1 let $H$ be a fixed DV-cell of the arrangement $C=\left\{x^{1}, \ldots, x^{n}\right\} \in \mathcal{P}(K, n)$, $n \in \mathbb{N}$, with respect to $x^{n}=0$ and let $\phi, y^{j}, L$ be defined as in definition 3.1.1. Now for $\rho \in(0, \sqrt{2})$ let $D_{\rho}=H \cap(\operatorname{conv}(C)+\rho K)$ and $U(\phi)=\operatorname{conv}\left\{0,2 y^{1} / f\left(y^{1}\right)\right.$, $\left.2 y^{2} / f\left(y^{2}\right)\right\} \cap H$. With respect to $U(\phi)$ we define $D_{\rho}^{i}, 1 \leq i \leq 4$, as in definition 3.1.2. Since $C \in \mathcal{P}(K, n)$, we have $\left|x^{j}\right| \geq 2 / f\left(y^{j}\right)$ and thus $2 y^{j} / f\left(y^{j}\right) \in \operatorname{conv}(C)$, $1 \leq j \leq n-1$. In particular

$$
\left\{x \in E^{d}:\left\langle x, y^{j}\right\rangle \leq 1 / f\left(y^{j}\right), 1 \leq j \leq n-1\right\} \subset H .
$$

Observe that $1 / R \leq f(v) \leq 1 / r$ for $v \in S^{d-1}$.

Next we give two auxiliary lemmas and then we transfer (generalize) the lemmas $3.1 .3,3.1 .4,3.1 .5,3.1 .6$ and 3.1 .10 to the centrally symmetric case.

Lemma 3.2.1 Let $K \in \mathcal{K}_{0}^{d}$ with distance function $f$, inradius $r$ and circumradius $R$. Then for $v, w \in S^{d-1}$ :

$$
|\langle v, w\rangle| \geq \cos (\phi) \Rightarrow f(v) \leq(1+\phi \cdot g(R / r)) \cdot f(w),
$$

where $g:[1, \infty) \rightarrow \mathbb{R}^{\geq 0}$ is a monotonely increasing function with $g(1)=0$. 
Proof. Assume $\langle v, w\rangle \geq \cos (\phi)$ and let $f(w) \leq f(v)$. Further let $a \in E^{d}$ be a unit outward normal vector of a supporting hyperplane $S$ of $K$ with $v / f(v) \in$ $K \cap S$. Let $\langle a, v / f(v)\rangle=\gamma=\cos (\psi) / f(v)$. On account of $\langle v, w\rangle \geq \cos (\phi)$ and $\langle a, w / f(w)\rangle \leq\langle a, v / f(v)\rangle$ we obtain $\gamma f(w) \geq\langle a, w\rangle \geq \cos (\phi) \cos (\psi)-\sin (\phi)(1-$ $\left.\cos ^{2}(\psi)\right)^{1 / 2}$ or

$$
1 \geq \frac{f(v)}{f(w)}\left(\cos (\phi)-\sin (\phi) \sqrt{(1 / \cos (\psi))^{2}-1}\right) .
$$

Now, $\cos (\psi) \geq r / R, f(v) / f(w) \leq R / r$ and thus

$$
\frac{f(v)}{f(w)} \leq \begin{cases}\left(\cos (\phi)-\sin (\phi) \sqrt{(R / r)^{2}-1}\right)^{-1} & : \phi \leq \arccos \left((r / R)^{2}\right)-\arccos (r / R), \\ R / r & : \text { else. }\end{cases}
$$

From this it is not hard to deduce an appropriate function $g$.

For abbreviation we write

$$
\begin{aligned}
\bar{R} & =R / r \\
\nu(\phi, \bar{R}) & =1+\phi g(\bar{R}), \\
\gamma(\rho, \phi, \bar{R}) & =(1-(\rho \bar{R}) \sin (\phi)) / \nu(\phi, \bar{R}) .
\end{aligned}
$$

Lemma 3.2.2 Let $\sin (\phi) \leq 1 /(\sqrt{2} \cdot \bar{R})$. Then for $\rho \leq \sqrt{2}$ and $i=1,2$ :

$$
\gamma(\rho, \phi, \bar{R}) \cdot \operatorname{conv}\left\{0, y^{i} / f\left(y^{i}\right)\right\}+\rho R\left(\left(y^{i}\right)^{\perp} \cap B^{d}\right) \subset H .
$$

Proof. Apparently, this statement is closely related to lemma 3.1.4, but in contrast to lemma 3.1.4 we can not make use of lemma 3.1.1 because in general $y^{j} / f\left(y^{j}\right) \notin r S^{d-1}$. Now, let $v_{y^{i}} \in\left(\left(y^{i}\right)^{\perp} \cap B^{d}\right), i=1,2$. By the definition of $\phi$ we have $\left\langle y^{j}, v_{y^{i}}\right\rangle \leq \sin (\phi), 1 \leq j \leq n-1$, (cf. lemma 3.1.3) and on account of the definition of $\nu(\phi, \bar{R})$ it holds $\left\langle\lambda y^{i} / f\left(y^{i}\right)+\rho R v_{y^{i}}, y^{j}\right\rangle \leq 1 / f\left(y^{j}\right), i=1,2$, if $\lambda \leq \gamma(\rho, \phi, \bar{R})$. Hence by (3.2.1) we get (3.2.2).

To obtain appropriate versions of lemma 3.1.3 and lemma 3.1.10 we note that $r B^{d} \subset K \cap H$ and therefore, lemma 3.2.3, 3.2.4 follow immediately from lemma $3.1 .3,3.1 .10$, respectively.

Lemma 3.2.3 Let $\sin (\phi) \leq 1 /(\sqrt{2} \cdot \bar{R})$. Then for $\rho \leq \sqrt{2}$ :

$$
V\left(D_{\rho}^{1}\right) \geq r^{d} \cdot \frac{\phi}{2} \cdot c_{1}(\rho, 1 / \sin (\phi)-\rho)^{2} \rho^{d-2} \cdot \kappa_{d-2}
$$

Lemma 3.2.4 Let $\phi>0$ and $\rho_{1}>0$ such that $d \geq d_{0}\left(\rho_{1}\right)=\max \left\{4,\left(4-\rho_{1}^{2}\right) /(2-\right.$ $\left.\left.\rho_{1}^{2}\right)\right\}$. Then for $\rho<\rho_{1}$ and $\epsilon>0$ with $\rho_{1}=\rho+\epsilon$ :

$$
V\left(D_{\rho}^{1}\right) \geq r^{d} \frac{\phi}{2} \cdot c_{1}(\rho, \epsilon)^{2} \rho^{d-2} \cdot \frac{\kappa_{d-2}}{1+c\left(\rho_{1}\right)} .
$$


The next two lemmas correspond to lemma 3.1.4 and lemma 3.1.5. Since in general the set $\left\{x \in D_{\rho}^{2}:\left\langle y^{i}, x\right\rangle=\gamma\right\}$ for $\gamma \in\left[0, \gamma(\rho, \phi, \bar{R}) / f\left(y^{i}\right)\right]$ does not contain a set of volume $\rho^{d-1} V\left(K \mid\left(y^{i}\right)^{\perp}\right) / 2$ as in the case $K=B^{d}$ we have to evaluate these sections more carefully. To this end we distinguish two cases depending on the sign of $\left\langle y^{1}, y^{2}\right\rangle$ and define some functionals which are helpful for the estimates.

Definition 3.2.1 Let $u \in S^{d-1}$. For $y \in\left(K \mid u^{\perp}\right)$ let

$$
\begin{aligned}
\lambda(y, u) & =\min \{|\lambda|: \lambda \in \mathbb{R} \text { and } y+\lambda u \in K\}, \\
s(u) & =\max \left\{\lambda(y, u): y \in\left(K \mid u^{\perp}\right)\right\}, \\
\rho_{K} & =\max \left\{1 /(s(u) f(u)): u \in S^{d-1}\right\}, \\
s(y, u) & =\left\{\begin{array}{l}
-\lambda(y, u): \text { if } y-\lambda(y, u) u \in K \\
\lambda(y, u): \text { if } y+\lambda(y, u) u \in K .
\end{array}\right.
\end{aligned}
$$

Obviously, $\lambda(y, u) \leq \sqrt{R^{2}-|y|^{2}}$. For $|y| \leq r$ the point $y$ belongs to $K$ and thus $\lambda(y, u)=0$. Hence

$$
|s(u)| \leq \sqrt{R^{2}-r^{2}} \text { and } \rho_{K} \geq r / \sqrt{R^{2}-r^{2}}
$$

From the definition we have $s(y, u)=-s(-y, u)$. In the case $R=r$ we obtain $s(u)=0$ and we may set $\rho_{K}=\infty$. Further let $u_{K} \in S^{d-1}$ such that $S_{n}\left(K, u_{K}\right)$ is a densest sausage configuration (see section 2.1). We set

$$
\beta(K)=V\left(K \mid u_{K}^{\perp}\right) / f\left(u_{K}\right)
$$

Lemma 3.2.5 Let $\left\langle y^{1}, y^{2}\right\rangle \leq-\cos (\phi)$ and let $\phi$ satisfy: $\sin (\phi) \leq 1 /(\sqrt{2} \cdot \bar{R})$, and $\nu(\phi, \bar{R}) \leq 2 \cos (\phi)$. Then for $\rho \leq \min \left\{\sqrt{2}, \rho_{K}\right\}$ :

$$
V\left(D_{\rho}^{2}\right) \geq \gamma(\rho, \phi, \bar{R})\left(\rho^{d-1} \beta(K)-2 \frac{\sin (\phi)}{\cos (\phi)} \sqrt{R^{2}-r^{2}} \rho^{d-1} R^{d-1} \kappa_{d-2}\right) .
$$

Proof. For $i=1,2$ and $\gamma \in\left[0, \gamma(\rho, \phi, \bar{R}) / f\left(y^{i}\right)\right]$ let $M_{\gamma}^{i}(\rho)=\{x \in \operatorname{conv}(C)+\rho K$ : $\left.\left\langle y^{i}, x\right\rangle=\gamma,\left\langle z^{i}, x\right\rangle \geq 0,\left|x-\gamma y^{i}\right| \leq \rho R\right\}$ where $z^{i} \in L$ denotes the outward unit normal vectors of the edges $\operatorname{conv}\left\{0,2 y^{i} / f\left(y^{i}\right)\right\}$ with respect to $\operatorname{conv}\left\{0,2 y^{1} / f\left(y^{1}\right)\right.$, $\left.2 y^{2} / f\left(y^{2}\right)\right\}$. In the following we show

$$
V\left(M_{\gamma}^{i}(\rho)\right) \geq \rho^{d-1} \frac{V\left(K \mid\left(y^{i}\right)^{\perp}\right)}{2}-\frac{\rho s\left(y^{i}\right) \sin (\phi)}{\cos (\phi)}(\rho R)^{d-2} \kappa_{d-2} .
$$

By (3.2.2) we have $M_{\gamma}^{i}(\rho) \subset D_{\rho}^{2}$ and on account of the definition of $s\left(y^{i}\right)$ inequality (3.2.4) implies the assertion. For the proof of (3.2.4) we only consider the case 
$i=1$; the other case can be treated similarly. Before we start we introduce some sets:

$$
\begin{aligned}
T & =\left\{x \in \rho\left(K \mid\left(y^{1}\right)^{\perp}\right):\left\langle z^{1}, x\right\rangle \geq 0\right\} \\
T^{\leq 0} & =\left\{x \in T: s\left(x / \rho, y^{1}\right) \leq 0\right\} \\
T^{>0} & =\left\{x \in T: s\left(x / \rho, y^{1}\right)>0\right\} \\
M_{\gamma} & =\left\{x \in \operatorname{conv}(C)+\rho K:\left\langle y^{1}, x\right\rangle=\gamma\right\}, \quad M_{\gamma}^{0}=M_{\gamma}^{1}(\rho)-\gamma y^{1}, \\
\alpha(x, \phi, \gamma) & =\rho \cdot s\left(x / \rho, y^{1}\right) \frac{2 \sin (\phi)}{\gamma f\left(y^{2}\right)+2 \cos (\phi)}, \quad \alpha(\phi)=\rho \cdot s\left(y^{1}\right) \frac{\sin (\phi)}{\cos (\phi)} .
\end{aligned}
$$

Obviously we have $V(T)=\rho^{d-1} V\left(K \mid\left(y^{1}\right)^{\perp}\right) / 2$ and $\alpha(\phi) \geq \alpha(x, \phi, \gamma)$. First we claim

$$
T^{\leq 0}+\gamma y^{1} \subset M_{\gamma}^{1}(\rho) .
$$

To prove this, it suffices to show $T^{\leq 0}+\gamma y^{1} \subset \operatorname{conv}(C)+\rho K$. Let $x \in T^{\leq 0}$ and let $\mu=\left(\rho \cdot s\left(x / \rho, y^{1}\right)+\left\langle x^{1}, y^{1}\right\rangle-\gamma\right) /\left\langle x^{1}, y^{1}\right\rangle$. Since $\rho \cdot s\left(x / \rho, y^{1}\right)-\gamma \leq 0$ we have $\mu \leq 1$. Further $\rho \leq \rho_{K}$ yields $\rho \cdot s\left(x / \rho, y^{1}\right) \geq-1 / f\left(y^{1}\right)$ and on account of $\gamma \leq 1 / f\left(y^{1}\right),\left\langle x^{1}, y^{1}\right\rangle=\left|x^{1}\right| \geq 2 / f\left(y^{1}\right)$ it follows $\mu \geq 0$. Now, $x+\gamma y^{1}=$ $\mu\left(x+\rho s\left(x / \rho, y^{1}\right) y^{1}\right)+(1-\mu)\left(x+\rho s\left(x / \rho, y^{1}\right) y^{1}+x^{1}\right) \in \operatorname{conv}(C)+\rho K$. This shows (3.2.5). Next we claim:

$$
x \in T^{>0} \Rightarrow x-\alpha(x, \phi, \gamma) z^{1}+\gamma y^{1} \in M_{\gamma} .
$$

Let $x \in T^{>0}$ and let $\mu=\left(\rho \cdot s\left(x / \rho, y^{1}\right) f\left(y^{2}\right)\right) /\left(\gamma f\left(y^{2}\right)+2 \cos (\phi)\right)$. It is clear that $\mu \geq 0$. By the definition of $\rho_{K}$ and by the choice of $\phi$ we obtain $\rho$. $s\left(x / \rho, y^{1}\right) f\left(y^{2}\right) \leq f\left(y^{2}\right) / f\left(y^{1}\right) \leq \nu(\phi, \bar{R}) \leq 2 \cos (\phi)$, where the second inequality follows from lemma 3.2.1. Hence $\mu \leq 1$ and on account of $y^{2}=-\cos (\phi) y^{1}-$ $\sin (\phi) z^{1}$ we get $x-\alpha(x, \phi, \gamma) z^{1}+\gamma y^{1}=\mu\left(2 y^{2} / f\left(y^{2}\right)+x+\rho s\left(x / \rho, y^{1}\right) y^{1}\right)+(1-$ $\mu)\left(\gamma y^{1}+x+\rho s\left(x / \rho, y^{1}\right) y^{1}\right) \in \operatorname{conv}(C)+\rho K$, which implies (3.2.6).

Now, let $G$ be the orthogonal projection of $T$ onto the hyperplane $\{x \in$ $\left.E^{d}:\left\langle z^{1}, x\right\rangle=0\right\}$. For $x \in G$ let $\bar{v}_{x}=\max \left\{v \in \mathbb{R}: x+v z^{1} \in M_{\gamma}^{0}\right\}$, $\underline{v}_{x}=\min \left\{v \in \mathbb{R}: x+v z^{1} \in M_{\gamma}^{0}\right\}$ and let $\bar{\sigma}_{x}, \underline{\sigma}_{x}$ be defined in the same way with respect to $T$ instead of $M_{\gamma}^{0}$. Since $V\left(M_{\gamma}^{1}(\rho)\right) \geq \int_{G} \bar{v}_{x}-\underline{v}_{x} d x$ and $\rho^{d-1} V\left(K \mid\left(y^{1}\right)^{\perp}\right) / 2=V(T)=\int_{G} \bar{\sigma}_{x}-\underline{\sigma}_{x} d x$ it suffices to show:

$$
\bar{v}_{x}-\underline{v}_{x} \geq \bar{\sigma}_{x}-\underline{\sigma}_{x}-\alpha(\phi), \quad x \in G .
$$

We note that if $\left\langle z^{1}, x\right\rangle-\alpha(x, \phi, \gamma) \geq 0$ holds for $x \in T^{>0}$ then we also have $x-\alpha(x, \phi, \gamma) z^{1}+\gamma y^{1} \in M_{\gamma}^{1}(\rho)$. Thus (3.2.7) follows immediately from (3.2.5), (3.2.6) and the convexity of $M_{\gamma}^{1}(\rho)$.

Lemma 3.2.6 Let $\sin (\phi) \leq 1 /(\sqrt{2} \cdot \bar{R}), \phi<\pi / 3$ and $\left\langle y^{1}, y^{2}\right\rangle>0$. Then for $\rho \leq \min \left\{\sqrt{2}, \rho_{K}\right\}$

$$
V\left(D_{\rho}^{2}\right)+V\left(D_{\rho}^{3}\right) \geq \rho^{d} \frac{V(K)}{2}+\gamma(\rho, \phi, \bar{R}) \rho^{d-1} \beta(K)-\frac{\phi}{\pi}(\rho R)^{d} \kappa_{d} .
$$


Proof. Again let $z^{i} \in L, i=1,2$, be the outward unit normal vectors of $\operatorname{conv}\left\{0,2 y^{i} / f\left(y^{i}\right)\right\}$ with respect to $\operatorname{conv}\left\{0,2 y^{1} / f\left(y^{1}\right), 2 y^{2} / f\left(y^{2}\right)\right\}$. We study the sets:

$$
\begin{aligned}
G^{r} & =\left\{x \in \rho K:\left\langle y^{2}, x\right\rangle>0 \wedge\left\langle y^{1}, x\right\rangle \leq 0\right\}, \\
G^{l} & =\left\{x \in \rho K:\left\langle y^{2}, x\right\rangle \leq 0 \wedge\left\langle y^{1}, x\right\rangle \leq 0\right\} \quad \text { and for } i=1,2 \\
T_{i}^{r} & =\left\{x \in \operatorname{conv}\left(\rho K \cup x^{i}+\rho K\right): 0 \leq\left\langle y^{i}, x\right\rangle \leq \gamma(\rho, \phi, \bar{R}) \wedge\left\langle z^{i}, x\right\rangle \geq 0\right\}, \\
T_{i}^{l} & =\left\{x \in \operatorname{conv}\left(\rho K \cup x^{i}+\rho K\right):\left\langle y^{i}, x\right\rangle \leq 0 \wedge\left\langle z^{i}, x\right\rangle \leq 0 \wedge x \notin \rho K\right\} .
\end{aligned}
$$

Obviously, $G^{l} \subset D_{\rho}^{3}$ and on account of (3.2.2) we get $T_{i}^{r} \subset D_{\rho}^{2}$. First we show that $T_{i}^{l}$ belongs to $D_{\rho}^{2} \cup D_{\rho}^{3}$. Let $x \in T_{i}^{l}$. Then $x \in \operatorname{conv}\left(\rho R B^{d} \cup x^{i}+\rho R B^{d}\right)$ and $\left\langle y^{i}, x\right\rangle \leq 0$ yields $|x| \leq \rho R$. Since $\phi<\pi / 3$ we have $\left\langle y^{i}, y^{j}\right\rangle \geq \cos (\phi)$ for $1 \leq j \leq n-1$ and so $\left\langle y^{j}, x\right\rangle \leq \sin (\phi)|x| \leq \sin (\phi) \rho R \leq r$. Here the last inequality follows by the choice of $\phi$. Now $r \leq 1 / f\left(y^{j}\right)$ and from (3.2.1) we obtain $x \in H$ which shows $x \in D_{\rho}^{2} \cup D_{\rho}^{3}$. On account of $V\left(G^{l} \cup G^{r}\right)=\rho^{d} V(K) / 2$ and $\operatorname{dim}\left(G^{l} \cap\left(T_{i}^{r} \cup T_{i}^{l}\right)\right) \leq d-1, \operatorname{dim}\left(T_{1}^{l} \cap T_{2}^{l}\right) \leq d-1, \operatorname{dim}\left(T_{1}^{r} \cap T_{2}^{r}\right) \leq d-1$ we may write

$$
\begin{aligned}
& V\left(D_{\rho}^{2} \cup D_{\rho}^{3}\right) \geq V\left(G^{l} \cup\left(T_{1}^{r} \cup T_{1}^{l}\right) \cup\left(T_{2}^{r} \cup T_{2}^{l}\right)\right) \\
\geq & V\left(G^{l}\right)+V\left(T_{1}^{r} \cup T_{1}^{l}\right)+V\left(T_{2}^{r} \cup T_{2}^{l}\right)-V^{d}\left(T_{1}^{l} \cap T_{2}^{r}\right)-V^{d}\left(T_{1}^{r} \cap T_{2}^{l}\right) \\
= & \rho^{d} \frac{V(K)}{2}-V\left(G^{r}\right)-V^{d}\left(T_{1}^{l} \cap T_{2}^{r}\right)-V^{d}\left(T_{1}^{r} \cap T_{2}^{l}\right)+\sum_{i=1}^{2} V\left(T_{i}^{r} \cup T_{i}^{l}\right) .
\end{aligned}
$$

In the above inequalities $V^{d}(\cdot)$ denotes the volume with respect to the $d$-dimensional space. For $i=1,2$ we claim:

$$
V\left(T_{i}^{r} \cup T_{i}^{l}\right) \geq \gamma(\rho, \phi, \bar{R}) \cdot \rho^{d-1} \frac{V\left(K \mid\left(y^{i}\right)^{\perp}\right)}{2 f\left(y^{i}\right)} .
$$

To prove this, it suffices to show

$$
V\left(M_{\gamma}^{i}\right) \geq \rho^{d-1} \frac{V\left(K \mid\left(y^{i}\right)^{\perp}\right)}{2}
$$

for $M_{\gamma}^{i}=\left\{x \in T_{i}^{r}:\left\langle y^{i}, x\right\rangle=\gamma\right\} \cup\left\{x \in T_{i}^{l}:\left\langle y^{i}, x\right\rangle=-\gamma\right\}$ and $0 \leq \gamma \leq$ $\gamma(\rho, \phi, \bar{R}) / f\left(y^{i}\right)$. Let $x \in \rho\left(K \mid\left(y^{i}\right)^{\perp}\right)$ with $\left\langle z^{i}, x\right\rangle \geq 0$.

a) $\rho \cdot s\left(x / \rho, y^{i}\right) \leq \gamma$.

Let $\mu=\left(\left\langle x^{i}, y^{i}\right\rangle+\rho \cdot s\left(x / \rho, y^{i}\right)-\gamma\right) /\left\langle x^{i}, y^{i}\right\rangle$. As in the proof of (3.2.5) we may deduce $\mu \in[0,1]$ and obtain $x+\gamma y^{i}=\mu\left(x+\rho \cdot s\left(x / \rho, y^{i}\right) y^{i}\right)+(1-\mu)(x+\rho$. $\left.s\left(x / \rho, y^{i}\right) y^{i}+x^{i}\right) \in \operatorname{conv}\left\{\rho K \cup\left(x^{i}+\rho K\right)\right\}$. Hence $x+\gamma y^{i} \in T_{i}^{r}$ and $x+\gamma y^{i} \in M_{\gamma}^{i}$. b) $\rho \cdot s\left(x / \rho, y^{i}\right)>\gamma$.

Assume $x+\gamma y^{i} \in \rho K$. Then we have $s\left(x / \rho, y^{i}\right) \leq \gamma / \rho$ which contradicts 
the assumption. Hence $-x-\gamma y^{i} \notin \rho K$ and further $\left\langle z^{i},-x-\gamma y^{i}\right\rangle \leq 0$. Now let $\mu=\left(\left\langle x^{i}, y^{i}\right\rangle+\gamma-\rho \cdot s\left(x / \rho, y^{i}\right)\right) /\left\langle x^{i}, y^{i}\right\rangle$. Again it follows $\mu \in[0,1]$. Since $-x-\rho \cdot s\left(x / \rho, y^{i}\right) y^{i}=-x+\rho \cdot s\left(-x / \rho, y^{i}\right) y^{i} \in \rho K$ we obtain $-x-\gamma y^{i}=$ $\mu\left(-x-\rho \cdot s\left(x / \rho, y^{i}\right) y^{i}\right)+(1-\mu)\left(-x-\rho \cdot s\left(x / \rho, y^{i}\right) y^{i}+x^{i}\right) \in \operatorname{conv}\left(\rho K \cup x^{i}+\rho K\right)$. Thus we have $-x-\gamma y^{1} \in T_{i}^{l}$ and $-x-\gamma y^{i} \in M_{\gamma}^{i}$.

Altogether we get (3.2.10) and so (3.2.9). With regard to (3.2.8) it remains to show

$$
V\left(G^{r}\right)+V^{d}\left(T_{1}^{l} \cap T_{2}^{r}\right)+V^{d}\left(T_{1}^{r} \cap T_{2}^{l}\right) \leq \frac{\phi}{\pi}(\rho R)^{d} \kappa_{d} .
$$

But this follows immediately from $G^{r} \cap\left(T_{1}^{l} \cap T_{2}^{r}\right)=\emptyset, G^{r} \cup\left(T_{1}^{l} \cap T_{2}^{r}\right) \subset\{x \in$ $\left.E^{d}:|x| \leq \rho R \wedge\left\langle y^{2}, x\right\rangle \geq 0 \wedge\left\langle y^{1}, x\right\rangle \leq 0\right\}$, and $\left(T_{1}^{r} \cap T_{2}^{l}\right) \subset\left\{x \in E^{d}:|x| \leq\right.$ $\left.\rho R \wedge\left\langle y^{2}, x\right\rangle \leq 0 \wedge\left\langle y^{1}, x\right\rangle \geq 0\right\}$.

Finally we transfer lemma 3.1.6. To do this we need some more notation:

$$
\begin{aligned}
& \underline{c}_{2}(\phi, \bar{R})=\max \left\{\frac{2 \nu(\phi, \bar{R})-1+\sqrt{2} \cdot \bar{R} \sin (\phi) \nu(\phi, \bar{R})}{2 \nu(\phi, \bar{R})+2 \cos (\phi)}, \frac{\nu(\phi, \bar{R})}{2 \sqrt{2+2 \cos (\phi)}}\right\}, \\
& \bar{c}_{2}(\phi, \bar{R})=\min \left\{\frac{2 \cos (\phi)+1 / \nu(\phi, \bar{R})-\sqrt{2} \cdot \bar{R} \sin (\phi)}{2 \nu(\phi, \bar{R})+2 \cos (\phi)}, 1-\frac{\nu(\phi, \bar{R})}{2 \sqrt{2+2 \cos (\phi)}}\right\} .
\end{aligned}
$$

Lemma 3.2.7 Let $\left\langle y^{1}, y^{2}\right\rangle<0$ and let $\phi$ satisfy: $\phi<\pi / 3$ and $\underline{c}_{2}(\phi, \bar{R}) \leq$ $\bar{c}_{2}(\phi, \bar{R})$. Then for $\rho \leq \min \left\{\sqrt{2}, \rho_{K}\right\}$ :

$$
V\left(D_{\rho}^{4}\right) \geq\left(\bar{c}_{2}(\phi, \bar{R})-\underline{c}_{2}(\phi, \bar{R})\right) \cdot \frac{\sqrt{2+2 \cos (\phi)}}{\nu(\phi, \bar{R})} \cdot \rho^{d-1} \cdot \beta(K) .
$$

Proof. Let $\underline{u}(\phi, \bar{R})(\bar{u}(\phi, \bar{R}))$ be the first term in the definition of $\underline{c}_{2}(\phi, \bar{R})$ $\left(\bar{c}_{2}(\phi, \bar{R})\right)$ and let $w=\left(y^{1} / f\left(y^{1}\right)-y^{2} / f\left(y^{2}\right)\right) /\left|y^{1} / f\left(y^{1}\right)-y^{2} / f\left(y^{2}\right)\right|$. On account of the definition of $\nu(\phi, \bar{R})$ and (3.2.1) we obtain with the method used in the proof of lemma 3.1.6:

$$
\lambda \frac{2 y^{1}}{f\left(y^{1}\right)}+(1-\lambda) \frac{2 y^{2}}{f\left(y^{2}\right)}+R \rho\left(w^{\perp} \cap B^{d}\right) \subset H, \lambda \in[\underline{u}(\phi, \bar{R}), \bar{u}(\phi, \bar{R})] .
$$

By assumption the above interval is well defined. Without loss of generality we assume $f\left(y^{2}\right) \leq f\left(y^{1}\right)$ and thus $\left|y^{1} / f\left(y^{1}\right)-y^{2} / f\left(y^{2}\right)\right| \geq\left|y^{1}-y^{2}\right| / f\left(y^{1}\right)$. Since $\left|\left\langle y^{1}, w\right\rangle\right| \geq \cos (\phi)$ we obtain with respect to lemma 3.2.1:

$$
f(w) \cdot\left|\frac{y^{1}}{f\left(y^{1}\right)}-\frac{y^{2}}{f\left(y^{2}\right)}\right| \geq \frac{\left|y^{1}-y^{2}\right|}{f\left(y^{1}\right)} \cdot \frac{f\left(y^{1}\right)}{\nu(\phi, \bar{R})} \geq \frac{\sqrt{2+2 \cos (\phi)}}{\nu(\phi, \bar{R})} .
$$


Let $z^{3}$ be the outward unit normal vector of $\operatorname{conv}\left\{2 y^{1} / f\left(y^{1}\right), 2 y^{2} / f\left(y^{2}\right)\right\}$ with respect to $\operatorname{conv}\left\{0,2 y^{1} / f\left(y^{1}\right), 2 y^{2} / f\left(y^{2}\right)\right\}$ and for $\gamma \in\left[\underline{c}_{2}(\phi, \bar{R}), \bar{c}_{2}(\phi, \bar{R})\right]$ let

$$
\begin{aligned}
M_{\gamma}= & \left\{x \in \operatorname{conv}\left\{\left(2 y^{1} / f\left(y^{1}\right)+\rho K\right) \cup\left(2 y^{2} / f\left(y^{2}\right)+\rho K\right)\right\}:\right. \\
& \left.x=\gamma \frac{2 y^{1}}{f\left(y^{1}\right)}+(1-\gamma) \frac{2 y^{2}}{f\left(y^{2}\right)}+y \text { with }\langle w, y\rangle=0 \wedge\left\langle z^{3}, y\right\rangle \geq 0\right\} .
\end{aligned}
$$

From (3.2.11) we get $M_{\gamma} \subset D_{\rho}^{4}$ and we claim:

$$
V\left(M_{\gamma}\right) \geq \rho^{d-1} \frac{V\left(K \mid w^{\perp}\right)}{2} .
$$

Let $T=\left\{y \in \rho\left(K \mid w^{\perp}\right):\left\langle z^{3}, y\right\rangle \geq 0\right\}$. For $y \in T$ let $\mu=\gamma-(\rho \cdot s(y / \rho, w))$ $\left(2\left|y^{1} / f\left(y^{1}\right)-y^{2} / f\left(y^{2}\right)\right|\right)$. By (3.2.12), the choice of $\gamma$ and the definition of $\rho_{K}$ we have $\mu \in[0,1]$. Hence $\gamma 2 y^{1} / f\left(y^{1}\right)+(1-\gamma) 2 y^{2} / f\left(y^{2}\right)+y=\mu\left(2 y^{1} / f\left(y^{1}\right)+y+\rho\right.$. $s(y / \rho, w) w)+(1-\mu)\left(2 y^{2} / f\left(y^{2}\right)+y+\rho \cdot s(y / \rho, w) w\right) \in M_{\gamma}$. Thus $\gamma 2 y^{1} / f\left(y^{1}\right)+$ $(1-\gamma) 2 y^{2} / f\left(y^{2}\right)+T \subset M_{\gamma}$ and we obtain (3.2.13). Hence

$$
\begin{aligned}
V\left(D_{\rho}^{4}\right) & \geq\left(\bar{c}_{2}(\phi, \bar{R})-\underline{c}_{2}(\phi, \bar{R})\right) \cdot\left|\frac{2 y^{1}}{f\left(y^{1}\right)}-\frac{2 y^{2}}{f\left(y^{2}\right)}\right| \rho^{d-1} \frac{V\left(K \mid w^{\perp}\right)}{2} \\
& \geq\left(\bar{c}_{2}(\phi, \bar{R})-\underline{c}_{2}(\phi, \bar{R})\right) \cdot \frac{\left|y^{1}-y^{2}\right|}{\nu(\phi, \bar{R})} \cdot \rho^{d-1} \frac{V\left(K \mid w^{\perp}\right)}{f(w)} .
\end{aligned}
$$

Now we can start with the proof of theorem 3.2.1.

Proof of theorem 3.2.1. Let $\bar{\phi} \in(0, \pi / 2]$ satisfy $\bar{c}_{2}(\bar{\phi}, \bar{R}) \geq \underline{c}_{2}(\bar{\phi}, \bar{R}), \sin (\bar{\phi}) \leq$ $1 /(\sqrt{2} \cdot \bar{R})$ and $\nu(\bar{\phi}, \bar{R}) \leq 2 \cos (\bar{\phi})$. Set $\phi_{0}=\min \{\bar{\phi}, \pi / 3\}$. We remark that $\phi_{0}$ depends only on the ratio $R / r$.

As in the proof of theorem 3.1.1 we distinguish three cases depending on $\phi$ and the sign of $\left\langle y^{1}, y^{2}\right\rangle$. Since the proof is completely analogous to the proof of theorem 3.1.1 we only give the essential steps.

I). $\phi<\phi_{0}$ and the assumptions of lemma 3.2.6 hold. By lemma 3.2.3 and lemma 3.2 .6 we get for $\rho \leq \min \left\{\sqrt{2}, \rho_{K}\right\}$ :

$$
\begin{aligned}
V\left(D_{\rho}\right) & \geq V\left(D_{\rho}^{1}\right)+V\left(D_{\rho}^{2}\right)+V\left(D_{\rho}^{3}\right) \\
& \geq \frac{V(K)}{2} \rho^{d}+\beta(K) \rho^{d-1}+\phi \rho^{d-2} r^{d} \cdot h_{1}(\rho, \bar{R}, d)
\end{aligned}
$$

where $h_{1}$ is a function with the following properties: $h_{1}$ is continuous in $\rho$ and $\bar{R}$, monotonely decreasing in $\bar{R}$ and $h_{1}(0, \bar{R}, d)>0$. Thus there exists a maximal $\psi_{1}(\bar{R}, d) \in(0, \sqrt{2}]$ such that

$$
h_{1}(\rho, \bar{R}, d)>0, \quad \rho \in\left[0, \psi_{1}(\bar{R}, d)\right) \text { and } h_{1}\left(\psi_{1}(\bar{R}, d), \bar{R}, d\right) \geq 0 .
$$


II). $\phi<\phi_{0}$ and the assumptions of lemma 3.2.7 hold. By lemma 3.2.3, 3.2.5 and 3.2.7 we get for $\rho \leq\left\{\sqrt{2}, \rho_{K}\right\}$

$$
\begin{aligned}
V\left(D_{\rho}\right) & \geq V\left(D_{\rho}^{1}\right)+V\left(D_{\rho}^{2}\right)+V\left(D_{\rho}^{4}\right) \\
& \geq 2 \beta(K) \rho^{d-1}+\phi \rho^{d-2} r^{d} \cdot h_{2}(\rho, \bar{R}, d),
\end{aligned}
$$

for a certain function $h_{2}$ with the same properties as $h_{1}$. Thus there exists a maximal $\psi_{2}(\bar{R}, d) \in(0, \sqrt{2}]$ such that

$$
h_{2}(\rho, \bar{R}, d)>0, \quad \rho \in\left[0, \psi_{2}(\bar{R}, d)\right) \text { and } h_{2}\left(\psi_{2}(\bar{R}, d), \bar{R}, d\right) \geq 0 .
$$

III). Let $\phi \geq \phi_{0}$ and $\rho_{1}=\max \left\{\rho \in \mathbb{R}^{>0}: d_{0}(\rho) \leq d\right\}$. Then we get by lemma 3.2.4 for $\rho<\rho_{1}$ :

$$
\begin{aligned}
V\left(D_{\rho}\right) & \geq V\left(D_{\rho}^{1}\right) \geq r^{d} \frac{\phi}{2} \rho^{d-2} c_{1}\left(\rho, \rho_{1}-\rho\right)^{2} \frac{\kappa_{d-2}}{1+c\left(\rho_{1}\right)} \\
& \geq 2 \beta(K) \rho^{d-1}+r^{d} \rho^{d-2} \cdot h_{3}(\rho, \bar{R}, d)
\end{aligned}
$$

for a certain function $h_{3}$ with the same properties as $h_{1}$. Thus there exists a maximal $\psi_{3}(\bar{R}, d) \in\left(0, \rho_{1}\right]$ such that

$$
h_{3}(\rho, \bar{R}, d)>0, \quad \rho \in\left[0, \psi_{3}(\bar{R}, d)\right) \text { and } h_{3}\left(\psi_{3}(\bar{R}, d), \bar{R}, d\right) \geq 0 .
$$

Now, let $\psi(\bar{R}, d)=\min \left\{\psi_{1}(\bar{R}, d), \psi_{2}(\bar{R}, d), \psi_{3}(\bar{R}, d)\right\}$. As the first case occurs at most twice we have shown that for $\rho<\psi(\bar{R}, d)$

$$
V(\operatorname{conv}(C)+\rho K) \geq V\left(\operatorname{conv}\left(S_{n}(K)\right)+\rho K\right)
$$

with equality if and only if $C$ is densest sausage configuration of $K$ (compare the proof of theorem 3.1.1). Since the functions $\psi_{i}(\bar{R}, d)$ are monotonely decreasing in $\bar{R}$ we also have this property for $\psi(\bar{R}, d)$.

Assume $\bar{R}=1$. Thus $K$ is a ball with radius 1 , say. We obtain $\rho_{K}=\infty$, $g(\bar{R})=0, \nu(\phi, \bar{R})=1,(\rho \bar{R})=\rho$ and $\underline{c}_{2}(\phi, \bar{R})=c_{2}(\phi, \sqrt{2}), \bar{c}_{2}(\phi, \bar{R})=1-$ $c_{2}(\phi, \sqrt{2})$ (cf. proof of lemma 3.1.6). Thus lemmas 3.2.3 - 3.2.4 become the lemmas of the last section, where lemma 3.2.6 is a combination of lemma 3.1.4 and 3.1.5. Hence we can choose the functions $h_{i}(\rho, \bar{R}, d), 1 \leq i \leq 3$, in such a way that $\lim _{d \rightarrow \infty} \psi(1, d)=\sqrt{2}$.

Let us remark that the above proof can not be applied to arbitrary convex bodies. For instance, if we consider the configuration in figure 3.2.1 we see that the sets $D_{1}$ at the ends of the sausage have not the same volumes. Hence we can not use our local approach (cf. (3.1.4)). 


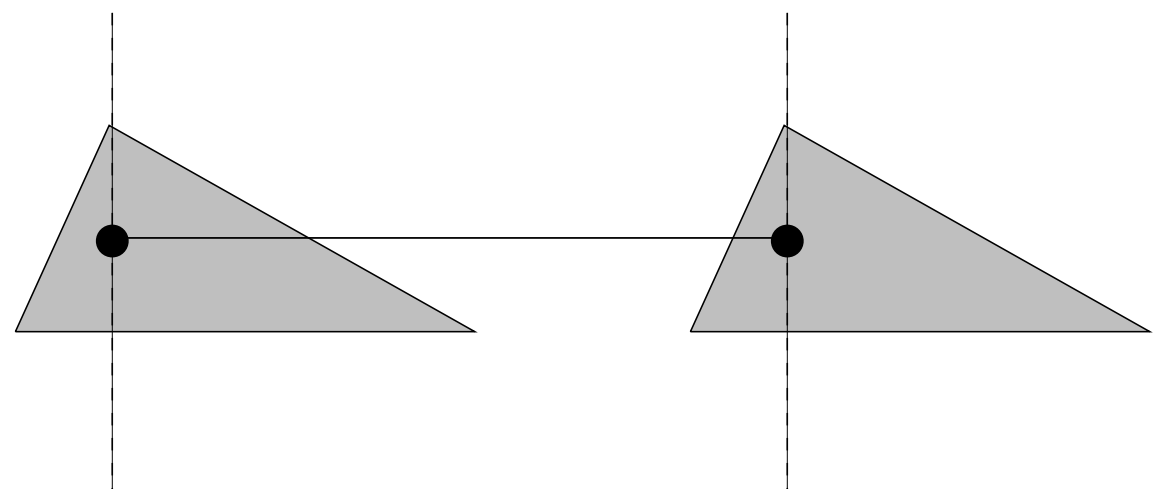

Figure 3.2.1. 


\section{The Sausage conjecture}

Based on the proof of theorem 3.1.1 Betke, Henk \& Wills gave for the "sausage" dimension $d(1)$ the upper bound 13,387 (cf. [BHW94a]). Here we prove that the sausage conjecture is true in dimensions $\geq 45$.

We use the notation introduced in section 3.1, i.e., $C=\left\{x^{1}, \ldots, x^{n}\right\}$ is a fixed packing set for the $d$-dimensional ball $B^{d}$ and $H$ is the DirichleT-VoronoÏ cell of the point $x^{n}=0$ with respect to $C$. Furthermore, let $D_{1}=H \cap(\operatorname{conv}(C)+$ $B^{d}$ ) and $\phi, y^{1}, y^{2}, L, U(\phi), D_{1}^{1}, D_{1}^{2}, D_{1}^{3}, D_{1}^{4}$ be defined as in definition 3.1 .1 and definition 3.1.2.

The proof of theorem 3.1.1 for $\rho=1$ can briefly be summarized as:

- If the angle $\phi$ is small then the facet defining hyperplanes of the DIRICHLET-VoronoÏ cell $H$ can not cut off to much from the set $U(\phi)+B^{d}$. Hence the volume of the sausage part of $D_{1}$ is of order $\kappa_{d-1}$ and the non-sausage part is of order $\kappa_{d-2}$. As $\kappa_{d-2} / \kappa_{d-1} \rightarrow \infty, d \rightarrow \infty$, we find a dimension where $V\left(D_{1}\right)$ is large enough (cf. lemmas 3.1.3 - 3.1.6).

- Independently of the angle $\phi$ we know that most of the $(d-3)$-dimensional sphere $L^{\perp} \cap \mu S^{d-1}$ of radius $\mu>1$ is contained in the cell $H$. So if $\phi$ is large we can find by the convexity of $H$ "many" points $w$ in $U(\phi)$ such that the volume of the section $\left(w+\left(L^{\perp} \cap B^{d}\right)\right) \cap H$ is of order $\kappa_{d-2}$ and hence $V\left(D_{1}\right) \geq 2 \kappa_{d-1}$ for large dimensions (cf. lemma 3.1.10).

To show that the sausage conjecture holds for dimensions $\geq 45$ we use the following refinements and supplements to the above arguments:

- Suppose the angle $\phi$ is small and there is a facet defining hyperplane of $H$ which has a non empty intersection with $U(\phi)+\left(L^{\perp} \cap B^{d}\right)$. Say the point of the configuration corresponding to this hyperplane is $x^{3}$. We call $x^{3}$ a perturbing point. Then, beside the volume related to $\left(U(\phi)+B^{d}\right) \cap H$, we find extra volume in the cell $H$ which belongs to the section $\left(\operatorname{conv}\left\{0, x^{3}\right\}+\right.$ $\left.B^{d}\right) \cap H$. A careful analysis of the relation between perturbing points and extra volume will be given in section 4.3 . 
- Instead of estimating the volume of the section $\left(w+\left(L^{\perp} \cap B^{d}\right)\right) \cap H, w \in$ $U(\phi)$, by the spherical volume of $\left(L^{\perp} \cap \mu S^{d-1}\right) \cap H, \mu>1$, (cf. lemma 3.1.10) we calculate for each point $w$ the volume of $\left(w+\left(L^{\perp} \cap S^{d-1}\right)\right) \cap H$ by a method similar to that one used in lemma 3.1.10. To this end we will give in section 4.1 an extension of lemma 3.1.7.

- For certain large $\phi$ we consider instead of the 2-dimensional set $U(\phi)$ a suitable 3-dimensional set $U(\alpha, \beta, \gamma)$. The necessary definitions as well as an estimate of $V\left(\left(U(\alpha, \beta, \gamma)+B^{d}\right) \cap H\right)$ will be given in section 4.2.

Finally in section 4.4 we will give the proof of:

"Theorem 45" Let $d \geq 45, n \in \mathbb{N}$, and $C \in \mathcal{P}\left(B^{d}, n\right)$. Then

$$
V\left(\operatorname{conv}(C)+B^{d}\right) \geq V\left(\operatorname{conv}\left(S_{n}\left(B^{d}\right)\right)+B^{d}\right)
$$

with equality if and only if $C$ is a sausage configuration.

\subsection{An extension of lemma 3.1.7}

In the proof of theorem 3.1.1 we derived from an estimate of the spherical volume $V_{*}\left(\left(L^{\perp} \cap \rho^{\prime} S^{d-1}\right) \cap H\right)$ a lower bound for $V_{*}\left(\left(w+\left(L^{\perp} \cap \rho S^{d-1}\right)\right) \cap H\right)$, $w \in U(\phi)$ and $\rho<\rho^{\prime}$. Here we describe a method which allows us to calculate $V_{*}\left(\left(w+\left(L^{\perp} \cap S^{d-1}\right)\right) \cap H\right)$ in a more direct and efficient way. Furthermore, based on this method we also obtain a "good" bound for $V\left(D_{1}^{2}\right)$.

To be more precise we need some elementary notation from the theory of convex polytopes (cf. [Grü67]). For a non empty $n$-dimensional face $F$ of a $p$-dimensional polytope $P \subset E^{d}$ the normal cone $N(P, F)$ is the cone generated by all vectors $v \in E^{d}$ with the property that there exists a $\nu \in \mathbb{R}$ with $F=P \cap\left\{x \in E^{d}:\langle v, x\rangle=\nu\right\}$. The dimension of the normal cone is $d-n$. In particular, $F+N(P, F)$ is the set of all points $x \in E^{d}$ such that the nearest point of $x$ with respect to $P$ belongs to $F$. The ratio of the spherical volume of $N(P, F) \cap S^{d-1}$ to $V_{*}\left(S^{d-n}\right)$ is called the external angle of $F$ and is denoted by $\theta(P, F)$.

Moreover we define some functions which will be used in the forthcoming estimates:

Definition 4.1.1 Let $r \in \mathbb{R}$ with $0 \leq r<1$ and $d, k, l, m \in \mathbb{N}$ such that $k+2 \leq$ $d-l+m$ and $k+2-m>\left(1+r^{2}\right) /\left(1-r^{2}\right)$. Let

$$
\begin{aligned}
a(r) & =\sqrt{1-r^{2}}, \\
c(k, m) & =\sqrt{\frac{2(k+2-m)}{k+3-m}-r^{2}-a(r)^{2}}=\sqrt{\frac{k+1-m}{k+3-m}},
\end{aligned}
$$




$$
\begin{aligned}
& \mu_{0}(k, m, r)=r / c(k, m),
\end{aligned}
$$

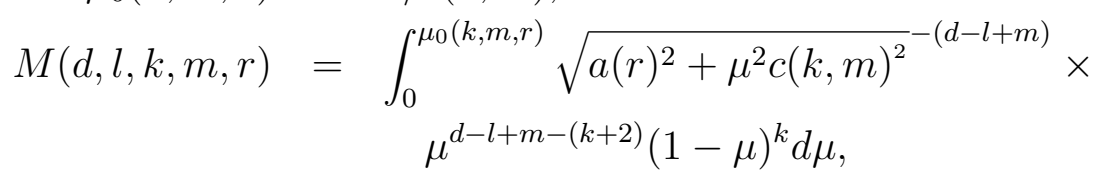

$$
\begin{aligned}
& \begin{array}{c}
K(d, l, k, m, r)=\int_{\mu_{0}(k, m, r)}^{1}{\sqrt{a(r)^{2}+\mu^{2} c(k, m)^{2}}}^{-(d-l+m)} \times \\
\mu^{d-l+m-(k+2)}(1-\mu)^{k} d \mu,
\end{array} \\
& Q(d, l, m, r)=\left\{k \in \mathbb{N}:\left(1+r^{2}\right) /\left(1-r^{2}\right)+m<k+2 \leq d-l+m\right\}, \\
& q(d, l, m, r)= \begin{cases}\infty & : Q(d, l, m, r)=\emptyset, \\
\min \left\{\frac{M(d, l, k, m, r)}{K(d, l, k, m, r)}: k \in Q(d, l, m, r)\right\}: & \text { otherwise. }\end{cases}
\end{aligned}
$$

The purpose of this section is to prove:

Lemma 4.1.1 Let $\hat{L} \subset E^{d}$ be an l-dimensional subspace and let $U \subset \hat{L}$ be an $l$-dimensional polytope with vertex 0 . Moreover, let $w \in U$ with $|w|<1$ such that there exists an $(l-m)$-dimensional face $F$ of $U$ with $0, w \in \operatorname{cl}(\operatorname{relint}(F))$. Then

$$
V_{*}\left(\left(w+\left(N(U, F) \cap S^{d-1}\right)\right) \cap H\right) \geq \theta(U, F) \cdot \frac{(d-l+m) \kappa_{d-l+m}}{1+q(d, l, m,|w|)} .
$$

Proof. Let $M_{w}=\left\{z \in N(U, F) \cap S^{d-1}: w+z \notin H\right\}$ and $K_{w}=\{z \in$ $\left.N(U, F) \cap S^{d-1}: w+z \in H\right\}$. By the definition of the external angle we have $V_{*}\left(M_{w}\right)+V_{*}\left(K_{w}\right)=\theta(U, F) \cdot(d-l+m) \kappa_{d-l+m}$ and thus

$$
V_{*}\left(K_{w}\right)=\theta(U, F) \frac{(d-l+m) \kappa_{d-l+m}}{1+V_{*}\left(M_{w}\right) / V_{*}\left(K_{w}\right)} .
$$

It remains to show

$$
V_{*}\left(M_{w}\right) / V_{*}\left(K_{w}\right) \leq q(d, l, m,|w|) .
$$

To this end we may assume $Q(d, l, m,|w|) \neq \emptyset$. To prove (4.1.2) we proceed as in the proof of lemma 3.1.7. First, we apply Rogers' dissection technique to the $(d-l+m)$-dimensional polyhedron $P=(w+N(U, F)) \cap H$ with respect to the reference point $c^{0}=w$. This means, we construct a dissection of the bounded polyhedron $P \cap W_{\sqrt{2}}^{d}$ into simplices $S$ of the form $S=\operatorname{conv}\left\{c^{0}, \ldots, c^{d-l+m}\right\}$ such that $c^{i}$ is contained in a $(d-l+m-i)$-face $G$ of $P \cap W_{\sqrt{2}}^{d}$ with $w \notin G, G$ contains $\operatorname{conv}\left\{c^{i}, \ldots, c^{d-l+m}\right\}$, and $c^{i}$ is the nearest point of $G$ to $c^{0}$.

Next we consider the distance of a point $c^{i}, i \geq 1$, of such a simplex to $w$. Obviously, if $c^{i}$ belongs to a face of $W_{\sqrt{2}}^{d}$ then we have $\left|c^{i}-w\right| \geq \sqrt{2-|w|^{2}}$. Now 
let $c^{i}$ be a point of a $(d-l+m-i)$-face $G$ of $P$. As the $(d-l)$-dimensional orthogonal complement of $\hat{L}$ is contained in $N(U, F)$ we have that for $i>m$ the point $c^{i}$ belongs to a $(d-(i-m))$-face of $H$. Clearly, for $1 \leq i \leq m$ the point $c^{i}$ lies at least in one facet of $H$. In view of a result of Rogers about the distance of $(d-i)$-faces of $H$ to the origin (cf. (3.1.13)) we get

$$
\left|c^{i}-w\right| \geq \begin{cases}\sqrt{1-|w|^{2}} & : 1 \leq i \leq m \\ \sqrt{2(i-m) /(i-m+1)-|w|^{2}}: & : m<i .\end{cases}
$$

Based on this dissection, (4.1.3) and the definition of the set $Q(d, l, m,|w|)$ we obtain analogously to the proof of lemma 3.1.7 that for each $k \in Q(d, l, m|w|)$ (cf. (3.1.15)):

$$
\frac{V_{*}\left(M_{w}\right)}{V_{*}\left(K_{w}\right)} \leq \frac{\int_{|\mu \bar{x}+(1-\mu) x|_{w} \leq 1}|\mu \bar{x}+(1-\mu) x|_{w}^{-(d-l+m)} \mu^{d-l+m-(k+2)}(1-\mu)^{k} d \mu}{\int_{|\mu \bar{x}+(1-\mu) x|_{w} \geq 1}|\mu \bar{x}+(1-\mu) x|_{w}^{-(d-l+m)} \mu^{d-l+m-(k+2)}(1-\mu)^{k} d \mu},
$$

where $|y|_{w}$ denotes the distance of the point $y$ to $w$ and $\bar{x} \in \operatorname{conv}\left\{c^{k+2}, \ldots\right.$, $\left.c^{d-l+m}\right\}, x \in \operatorname{conv}\left\{c^{1}, \ldots, c^{k+1}\right\}$ are appropriate points of a simplex $\operatorname{conv}\left\{c^{0}, \ldots\right.$, $\left.c^{d-l+m}\right\}$ of the above construction. By (4.1.3) and the choice of $k$ we have

$$
|x|_{w} \geq \sqrt{1-|w|^{2}}, \quad|\bar{x}|_{w} \geq \sqrt{2(k+2-m) /(k+3-m)-|w|^{2}}>1 .
$$

Since $|\mu \bar{x}+(1-\mu) x|_{w}$ is monotonely increasing in $\mu$ we may assume $|x|_{w}<1$. Then (4.1.4) is of the form

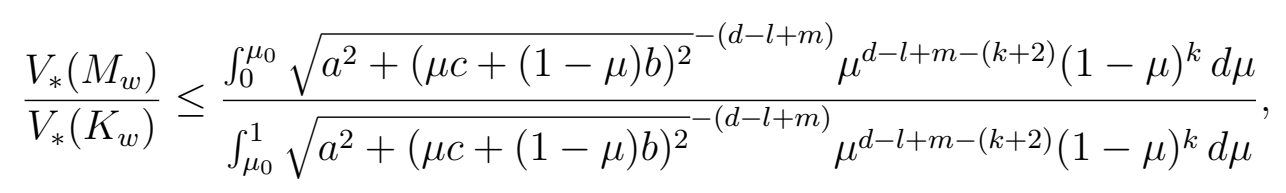

where $a \geq \alpha=\sqrt{1-|w|^{2}}$ denotes the distance of the line through $\bar{x}, x$ from $w, b$ is given by $a^{2}+b^{2}=|x|_{w}^{2}, c$ by $a^{2}+c^{2}=|\bar{x}|_{w}^{2}$ and $\mu_{0}$ is determined by $a^{2}+\left(\mu_{0} c+\left(1-\mu_{0}\right) b\right)^{2}=1$. But now (4.1.2) follows from lemma 3.1 .9 and definition 4.1 .1 with $\beta=1, \gamma=2(k+2-m) /(k+3-m)-|w|^{2}, \alpha=a(|w|)$, $b=0, c=c(k, m)$ and $\mu_{0}=\mu_{0}(k, m,|w|)$.

Remark 4.1.1 As $Q(d, l, m, r) \subset Q\left(d^{\prime}, l, m, r\right)$ for $d^{\prime} \geq d$ we see by lemma 3.1.9 that the function $q(d, l, m, r)$ is monotonely decreasing in $d$.

Instead of the spherical volume $V_{*}\left(\left(w+\left(N(U, F) \cap S^{d-1}\right)\right) \cap H\right)$ we are often interested in the volume $V\left(\left(w+\left(N(U, F) \cap B^{d}\right)\right) \cap H\right)$. Since

$$
V\left(\left(w+\left(N(U, F) \cap B^{d}\right)\right) \cap H\right)=\frac{1}{d-l+m} V_{*}\left(\left(w+\left(N(U, F) \cap S^{d-1}\right)\right) \cap H\right)
$$

we have: 
Corollary 4.1.1 Under the assumptions of lemma 4.1.1 one has

$$
V\left(\left(w+\left(N(U, F) \cap B^{d}\right)\right) \cap H\right) \geq \theta(U, F) \cdot \frac{\kappa_{d-l+m}}{1+q(d, l, m,|w|)}
$$

Furthermore, as an immediate consequence we obtain:

\section{Corollary 4.1.2}

$$
V\left(D_{1}^{1}\right) \geq \kappa_{d-2} \int_{U(\phi)} \frac{1}{1+q(d, 2,0,|w|)} d w, \quad V\left(D_{1}^{2}\right) \geq \kappa_{d-1} \int_{0}^{1} \frac{1}{1+q(d, 2,1, r)} d r .
$$

Proof. Let $U=\operatorname{conv}\left\{0,2 y^{1}, 2 y^{2}\right\}$. For $F=U$ we have $\theta(U, F)=1$ and $N(U, F)=L^{\perp}$. By the definition of $D_{1}^{1}$ and the normal cones we get

$$
\left(U(\phi)+\left(N(U, F) \cap B^{d}\right)\right) \cap H \subset D_{1}^{1} .
$$

On account of corollary 4.1.1 this implies the lower bound for $V\left(D_{1}^{1}\right)$. For the bound of $V\left(D_{1}^{2}\right)$ we note that

$$
\left(\operatorname{conv}\left\{0, y^{i}\right\}+\left(N\left(U, \operatorname{conv}\left\{0,2 y^{i}\right\}\right) \cap B^{d}\right)\right) \cap H \subset D_{1}^{2}
$$

and $\theta\left(U, \operatorname{conv}\left\{0,2 y^{i}\right\}\right)=1 / 2$ for $i=1,2$.

In the next section we will apply corollary 4.1 .1 to a 3 -dimensional subset of $\operatorname{conv}(C) \cap H$.

\subsection{3 -dimensional sections}

In the proof of theorem 3.1.1 we considered the parallel body of the 2-dimensional set $U(\phi)$. Here we introduce a 3 -dimensional subset $U(\alpha, \beta, \gamma)$ of $\operatorname{conv}(C)$ and we give an estimate for the volume part of $U(\alpha, \beta, \gamma)+B^{d}$ belonging to $H$.

We may assume that $x^{3} \in C$ is a vector of the configuration which has a maximal angle $\alpha \in[0, \pi / 2]$ with the plane $L=\operatorname{lin}\left\{y^{1}, y^{2}\right\}$, i.e.

$$
\left|\left(y^{3} \mid L\right)\right|=\min _{1 \leq i \leq n-1}\left\{\left|\left(y^{i} \mid L\right)\right|\right\} \text { and } \alpha=\arccos \left(\left|\left(y^{3} \mid L\right)\right|\right),
$$

where we always assume $\arccos (\cdot) \in[0, \pi]$. For simplification we assign the following coordinates to $y^{1}, y^{2}, y^{3}$ :

$$
\begin{aligned}
& y^{1}=(1,0,0, \ldots, 0)^{T} \\
& y^{2}=(\cos (\gamma), \sin (\gamma), 0, \ldots, 0)^{T}, \\
& y^{3}=(\cos (\alpha) \cos (\beta), \cos (\alpha) \sin (\beta), \sin (\alpha), 0, \ldots, 0)^{T},
\end{aligned}
$$


with $\beta \in[0,2 \pi] . \gamma \in[0, \pi]$ denotes the angle between $y^{1}$ and $y^{2}$. Clearly, the angles $\alpha, \beta, \gamma$ are not independent of each other and by (3.1.5) we find

$$
|\cos (\alpha) \cos (\beta)| \geq \cos (\phi), \quad|\cos (\alpha) \cos (\gamma-\beta)| \geq \cos (\phi) .
$$

So $\alpha \geq \pi / 3$ implies $\phi \geq \pi / 3$ and by (3.1.5) we have $|\cos (\gamma)|=\cos (\phi)$ for $\phi \geq \pi / 3$. Hence for $\alpha \geq \pi / 3$

$$
|\cos (\alpha) \cos (\beta)| \geq|\cos (\gamma)|, \quad|\cos (\alpha) \cos (\gamma-\beta)| \geq|\cos (\gamma)| .
$$

In the following we study some geometric quantities of the 3-dimensional set

$$
U(\alpha, \beta, \gamma)=\operatorname{conv}\left\{0,2 y^{1}, 2 y^{2}, 2 y^{3}\right\} \cap B^{d} \subset H .
$$

Let $f_{i, j}$ denotes the angle between $y^{i}$ and $y^{j}, 1 \leq i<j \leq 3$. Then

$$
f_{1,2}=\gamma, \quad f_{1,3}=\arccos (\cos (\alpha) \cos (\beta)) \text { and } f_{2,3}=\arccos (\cos (\alpha) \cos (\gamma-\beta)) .
$$

For $\alpha>0$ let $u_{i, j} \in \operatorname{lin}\left\{y^{1}, y^{2}, y^{3}\right\}, 1 \leq i<j \leq 3$, be the outward normal unit vector of the 2 -face $F_{i, j}=\operatorname{conv}\left\{0,2 y^{i}, 2 y^{j}\right\} \cap B^{d}$ of $U(\alpha, \beta, \gamma)$ :

$$
\begin{aligned}
u_{1,2}= & (0,0,-1,0, \ldots, 0)^{T} \\
u_{1,3}= & (0,-\sin (\alpha), \cos (\alpha) \sin (\beta), 0, \ldots, 0)^{T} / \sqrt{1-\cos ^{2}(\alpha) \cos ^{2}(\beta)}, \\
u_{2,3}= & (-\sin (\alpha) \sin (\gamma), \sin (\alpha) \cos (\gamma), \cos (\alpha) \sin (\gamma-\beta), 0, \ldots, 0)^{T} / \\
& \quad \sqrt{1-\cos ^{2}(\alpha) \cos ^{2}(\gamma-\beta)} .
\end{aligned}
$$

Finally let $g_{1,2}, g_{1,3}$ and $g_{2,3}$ denote the angle between the normal vectors $\left(u_{1,3}\right.$, $\left.u_{2,3}\right),\left(u_{1,2}, u_{2,3}\right)$ and $\left(u_{1,2}, u_{1,3}\right)$, respectively. We get

$$
\begin{aligned}
g_{1,2} & =\arccos \left(\frac{-\sin ^{2}(\alpha) \cos (\gamma)+\cos ^{2}(\alpha) \sin (\beta) \sin (\gamma-\beta)}{\sqrt{1-\cos ^{2}(\alpha) \cos ^{2}(\beta)} \sqrt{1-\cos ^{2}(\alpha) \cos ^{2}(\gamma-\beta)}}\right), \\
g_{1,3} & =\arccos \left(\frac{-\cos (\alpha) \sin (\gamma-\beta)}{\sqrt{1-\cos ^{2}(\alpha) \cos ^{2}(\gamma-\beta)}}\right), \\
g_{2,3} & =\arccos \left(\frac{-\cos (\alpha) \sin (\beta)}{\sqrt{1-\cos ^{2}(\alpha) \cos ^{2}(\beta)}}\right) .
\end{aligned}
$$

With these notation we obtain for $V\left(D_{1}\right)$ the lower bound:

Lemma 4.2.1 Let $\alpha \geq \pi / 3$ and let $h$ be the distance of $\operatorname{conv}\left\{2 y^{1}, 2 y^{2}, 2 y^{3}\right\}$ to the origin. Then

$$
V\left(D_{1}\right) \geq V\left(\left(U(\alpha, \beta, \gamma)+B^{d}\right) \cap H\right) \geq
$$




$$
\begin{aligned}
& \left(\frac{g_{1,2}+g_{1,3}+g_{2,3}}{2 \pi}\right) \cdot \kappa_{d-1} \int_{0}^{1} \frac{1}{1+q(d, 3,2, r)} d r \\
+ & \left(\frac{f_{1,2}+f_{1,3}+f_{2,3}}{2}\right) \cdot \kappa_{d-2} \int_{0}^{1} \frac{r}{1+q(d, 3,1, r)} d r \\
+ & \left(2 \pi-g_{1,2}-g_{1,3}-g_{2,3}\right) \cdot \kappa_{d-3} \int_{0}^{\min \{1, h\}} \frac{r^{2}}{1+q(d, 3,0, r)} d r .
\end{aligned}
$$

Proof. For abbreviation we set $\bar{U}=U(\alpha, \beta, \gamma)$ and $U=\operatorname{conv}\left\{0,2 y^{1}, 2 y^{2}, 2 y^{3}\right\}$. Obviously, we have $\left(\bar{U}+B^{d}\right) \cap H \subset D_{1}$ and by the definition of the normal cones follows:

$$
\begin{aligned}
& V\left(\left(\bar{U}+B^{d}\right) \cap H\right) \geq \sum_{i=1}^{3} \int_{\operatorname{conv}\left\{0, y^{i}\right\}} V\left(\left(w+\left(N\left(U, \operatorname{conv}\left\{0,2 y^{i}\right\}\right) \cap B^{d}\right)\right) \cap H\right) d w \\
& +\sum_{1 \leq i<j \leq 3} \int_{F_{i, j}} V\left(\left(w+\left(N\left(U, \operatorname{conv}\left\{0,2 y^{i}, 2 y^{j}\right\}\right) \cap B^{d}\right)\right) \cap H\right) d w \\
& +\int_{\bar{U}} V\left(\left(w+\left(N(U, U) \cap B^{d}\right)\right) \cap H\right) d w .
\end{aligned}
$$

From corollary 4.1.1 we obtain:

$$
\begin{aligned}
& V\left(\left(\bar{U}+B^{d}\right) \cap H\right) \geq \sum_{i=1}^{3} \theta\left(U, \operatorname{conv}\left\{0,2 y^{i}\right\}\right) \cdot \kappa_{d-1} \int_{\operatorname{conv}\left\{0, y^{i}\right\}} \frac{1}{1+q(d, 3,2,|w|)} d w \\
& +\sum_{1 \leq i<j \leq 3} \theta\left(U, \operatorname{conv}\left\{0,2 y^{i}, 2 y^{j}\right\}\right) \cdot \kappa_{d-2} \int_{F_{i, j}} \frac{1}{1+q(d, 3,1,|w|)} d w \\
& +\theta(U, U) \cdot \kappa_{d-3} \int_{\bar{U}} \frac{1}{1+q(d, 3,0,|w|)} d w .
\end{aligned}
$$

Now $\theta\left(U, \operatorname{conv}\left\{0,2 y^{i}\right\}\right)=g_{k, j} /(2 \pi), k, j \neq i, \theta\left(U, \operatorname{conv}\left\{0,2 y^{i}, 2 y^{j}\right\}\right)=1 / 2$ and $\theta(U, U)=1$. Since $\alpha \geq \pi / 3$ we have $f_{1,2}, f_{1,3}, f_{2,3} \in[\pi / 3,2 \pi / 3]$. Thus the intersection of the cone generated by $y^{i}, y^{j}$ with $B^{d}$ belongs to the 2 -face $F_{i, j}$. Hence

$$
\int_{F_{i, j}} \frac{1}{1+q(d, 3,1,|w|)} d w \geq f_{i, j} \int_{0}^{1} \frac{r}{1+q(d, 3,1, r)} d r
$$

Moreover, by the choice of $h$ we have

$$
\min \{1, h\}\left(\operatorname{cone}\left\{y^{1}, y^{2}, y^{3}\right\} \cap B^{d}\right) \subset \bar{U}
$$

and as $V_{*}\left(\operatorname{cone}\left\{y^{1}, y^{2}, y^{3}\right\} \cap S^{d-1}\right)=\left(2 \pi-g_{1,2}-g_{1,3}-g_{2,3}\right)$ (cf. [Sch50]) we get

$$
\int_{\bar{U}} \frac{1}{1+q(d, 3,0,|w|)} d w \geq\left(2 \pi-g_{1,2}-g_{1,3}-g_{2,3}\right) \int_{0}^{\min \{h, 1\}} \frac{r^{2}}{1+q(d, 3,0, r)} d r .
$$


Based on lemma 4.2.1 we give in the sequel a lower bound for $V\left(D_{1}\right)$ only depending on the angle $\alpha$ and the dimension. A lower bound for the distance $h$ is given by the distance $\eta(\alpha, \beta, \gamma)$ of the affine hull of $\left\{2 y^{1}, 2 y^{2}, 2 y^{3}\right\}$ to the origin:

$$
\begin{aligned}
h \geq \eta(\alpha, \beta, \gamma)= & (2 \sin (\alpha) \sin (\gamma)) \cdot\left((\sin (\alpha) \sin (\gamma))^{2}+(\sin (\alpha)(1-\cos (\gamma)))^{2}\right. \\
& \left.+(\sin (\gamma)-\cos (\alpha) \sin (\beta)+\cos (\alpha) \sin (\beta-\gamma))^{2}\right)^{-1 / 2} .
\end{aligned}
$$

Calculating the first and second partial derivatives with respect to $\beta$ shows that $\eta(\alpha, \beta, \gamma) \geq \min \{\eta(\alpha, \gamma / 2, \gamma), \eta(\alpha, \pi+\gamma / 2, \gamma)\}=\eta(\alpha, \pi+\gamma / 2, \gamma)$. Furthermore, it easy to see that for $\gamma \in[0, \pi], \alpha \in[0, \pi / 2]$ the function $\eta(\alpha, \pi+\gamma / 2, \gamma)$ is monotonely increasing in $\alpha$ and monotonely decreasing in $\gamma$. Since $\alpha \geq \pi / 3$ implies $\gamma \in[\pi / 3,2 \pi / 3]$ (cf. (4.2.2)) the distance is minimal for $\alpha=\pi / 3, \gamma=2 \pi / 3$ and $\beta=4 \pi / 3$. It follows:

$$
h \geq \eta(\pi / 3,4 \pi / 3,2 \pi / 3)=\sqrt{3 / 7}>0.65463, \quad \alpha \geq \pi / 3 .
$$

In order to estimate $V\left(D_{1}\right)$ we introduce the abbreviations:

$$
\begin{aligned}
w_{i}(d) & =\int_{0}^{1} \frac{r^{i-1}}{1+q(d, 3,3-i, r)} d r, \quad i=1,2 \\
w_{3}(d) & =\int_{0}^{\sqrt{3 / 7}} \frac{r^{2}}{1+q(d, 3,0, r)} d r
\end{aligned}
$$

and

$$
\begin{aligned}
f_{1}(\alpha, \beta, \gamma, d) & =\sum g_{i, j}\left(\frac{w_{1}(d) \cdot \kappa_{d-1}}{2 \pi}-w_{3}(d) \kappa_{d-3}\right) \\
& +2 \pi w_{3}(d) \kappa_{d-3}+\frac{\sum f_{i, j}}{2} w_{2}(d) \kappa_{d-2},
\end{aligned}
$$

where $\sum$ indicates the summation over $1 \leq i<j \leq 3$. By lemma 4.2 .1 and (4.2.3) we have

$$
V\left(D_{1}\right) \geq V\left(\left(U(\alpha, \beta, \gamma)+B^{d}\right) \cap H\right) \geq f_{1}(\alpha, \beta, \gamma, d), \quad \text { for } \alpha \geq \pi / 3 .
$$

We claim:

Theorem 4.2.1 Let $\pi / 3 \leq \alpha_{0} \leq \pi / 2$ and let $d$ satisfy

$$
\frac{w_{1}(d) \cdot \kappa_{d-1}}{2 \pi}-w_{3}(d) \kappa_{d-3} \leq 0 .
$$

Then for $\alpha \geq \alpha_{0}$ one has

$$
V\left(D_{1}\right) \geq f_{1}\left(\alpha_{0}, \gamma_{0} / 2, \gamma_{0}, d\right)
$$

where $\gamma_{0} \in[\pi / 3, \pi / 2]$ is given by $\cos \left(\alpha_{0}\right) \cos \left(\gamma_{0} / 2\right)=\cos \left(\gamma_{0}\right)$. 
Proof. It suffices to show that for $\alpha \geq \alpha_{0}$ and on account of the restriction (4.2.2) the function $f_{1}(\alpha, \beta, \gamma, d)$ is minimal for $\alpha=\alpha_{0}, \beta=\gamma_{0} / 2$ and $\gamma=\gamma_{0}$. To this end we study the behavior of the partial derivatives of $\sum f_{i, j}$ and $\sum g_{i, j}$. The calculations of the derivatives were carried out with help of the program Mathematica ${ }^{1}$, but all results were also verified "by hand" (see appendix). Since the necessary trigonometric transformations are rather tedious we omit details. With respect to $\gamma$ we obtain:

$$
\begin{aligned}
\frac{\partial \sum f_{i, j}}{\partial \gamma} & =\frac{\partial f_{1,2}}{\partial \gamma}+\frac{\partial f_{2,3}}{\partial \gamma}=1+\frac{\cos (\alpha) \sin (\gamma-\beta)}{\sqrt{1-\cos ^{2}(\alpha) \cos ^{2}(\gamma-\beta)}} \\
& =1+\frac{\cos (\alpha) \sin (\gamma-\beta)}{\sqrt{\sin ^{2}(\alpha)+\cos ^{2}(\alpha) \sin ^{2}(\gamma-\beta)}} \geq 0, \\
\frac{\partial \sum g_{i, j}}{\partial \gamma} & =\frac{\partial g_{1,2}}{\partial \gamma}+\frac{\partial g_{1,3}}{\partial \gamma} \\
& =\frac{-\sin (\alpha)}{1-\cos ^{2}(\alpha) \cos ^{2}(\gamma-\beta)}+\frac{\sin (\alpha) \cos (\alpha) \cos (\gamma-\beta)}{1-\cos ^{2}(\alpha) \cos ^{2}(\gamma-\beta)} \\
& =\frac{-\sin (\alpha)}{1+\cos (\alpha) \cos (\gamma-\beta)} \leq 0 .
\end{aligned}
$$

So for all $\alpha \in\left[\alpha_{0}, \pi / 2\right], \beta \in[0,2 \pi]$ the function $\sum f_{i, j}$ is monotonely increasing in $\gamma$ and $\sum g_{i, j}$ is monotonely decreasing in $\gamma$. By the choice of $d$ (cf. (4.2.5)) we get that $f_{1}(\alpha, \beta, \gamma, d)$ is monotonely increasing in $\gamma$. Hence we may choose $\gamma$ as small as possible, i.e. we may decrease $\gamma$ as long as (4.2.2) is satisfied. In any case we may assume that $f_{1}(\alpha, \beta, \gamma, d)$ attains its minimum for

$$
\gamma \leq \pi / 2
$$

Next we consider the partial derivatives with respect to $\beta$ and get:

$$
\begin{aligned}
\frac{\partial \sum f_{i, j}}{\partial \beta}= & \frac{\partial f_{1,3}}{\partial \beta}+\frac{\partial f_{2,3}}{\partial \beta} \\
= & \frac{\cos (\alpha) \sin (\beta)}{\sqrt{1-\cos ^{2}(\alpha) \cos ^{2}(\beta)}}-\frac{\cos (\alpha) \sin (\gamma-\beta)}{\sqrt{1-\cos ^{2}(\alpha) \cos ^{2}(\gamma-\beta)}}, \\
\frac{\partial \sum g_{i, j}}{\partial \beta}= & \frac{\partial g_{1,2}}{\partial \beta}+\frac{\partial g_{1,3}}{\partial \beta}+\frac{\partial g_{2,3}}{\partial \beta} \\
= & -\frac{\sin (\alpha) \cos ^{2}(\alpha) \sin (\gamma) \sin (\gamma-2 \beta)}{\left(1-\cos ^{2}(\alpha) \cos ^{2}(\beta)\right)\left(1-\cos ^{2}(\alpha) \cos ^{2}(\gamma-\beta)\right)} \\
& -\frac{\sin (\alpha) \cos (\alpha) \cos (\gamma-\beta)}{1-\cos ^{2}(\alpha) \cos ^{2}(\gamma-\beta)}+\frac{\sin (\alpha) \cos ^{2}(\alpha) \cos (\beta)}{1-\cos ^{2}(\alpha) \cos 2(\beta)} \\
= & \frac{2 \sin (\alpha) \cos (\alpha) \sin (\gamma / 2) \sin (\gamma / 2-\beta)}{(1+\cos (\alpha) \cos (\beta))(1+\cos (\alpha) \cos (\gamma-\beta))} .
\end{aligned}
$$

\footnotetext{
${ }^{1}$ (c) 1988,1991,1992 von Wolfram Research Inc.
} 
It is easy to see that

$$
\begin{array}{ll}
\frac{\partial \sum f_{i, j}}{\partial \beta} & \left\{\begin{array}{l}
\leq 0: 0 \leq \beta \leq \gamma / 2, \quad \pi+\gamma / 2 \leq \beta \leq 2 \pi, \\
=0: \beta=\gamma / 2, \quad \beta=\pi+\gamma / 2 \\
\geq 0: \gamma / 2 \leq \beta \leq \pi+\gamma / 2,
\end{array}\right. \\
\frac{\partial \sum g_{i, j}}{\partial \beta} & \left\{\begin{array}{l}
\geq 0: 0 \leq \beta \leq \gamma / 2, \quad \pi+\gamma / 2 \leq \beta \leq 2 \pi, \\
=0: \beta=\gamma / 2, \quad \beta=\pi+\gamma / 2, \\
\leq 0: \gamma / 2 \leq \beta \leq \pi+\gamma / 2 .
\end{array}\right.
\end{array}
$$

Thus for given $\alpha \in\left[\alpha_{0}, \pi / 2\right], \beta \in[0,2 \pi]$ and $\gamma \in[0, \pi / 2]$ we have (cf. (4.2.5)):

$$
f_{1}(\alpha, \beta, \gamma, d) \geq f_{1}(\alpha, \gamma / 2, \gamma, d)
$$

It remains to show that the combination $(\alpha, \gamma / 2, \gamma)$ satisfies the "angle restriction" (4.2.2). Now, (4.2.2) implies

$$
\cos (\alpha)(|\cos (\beta)|+|\cos (\beta-\gamma)|) \geq 2 \cos (\gamma) .
$$

From $\gamma \leq \pi / 2$ follows $|\cos (\beta)|+|\cos (\beta-\gamma)| \leq 2 \cos (\gamma / 2)$ and therefore, we get $\cos (\alpha) \cos (\gamma / 2) \geq \cos (\gamma)$.

Finally, for the partial derivatives with respect to $\alpha$ we find:

$$
\begin{aligned}
\frac{\partial \sum f_{i, j}}{\partial \alpha}(\alpha, \gamma / 2, \gamma) & =\left(\frac{\partial f_{1,3}}{\partial \alpha}+\frac{\partial f_{2,3}}{\partial \alpha}\right)(\alpha, \gamma / 2, \gamma) \\
& =2 \frac{\sin (\alpha) \cos (\gamma / 2)}{\sqrt{1-\cos ^{2}(\alpha) \cos ^{2}(\gamma / 2)}} \geq 0 \\
\frac{\partial \sum g_{i, j}}{\partial \alpha}(\alpha, \gamma / 2, \gamma) & =\left(\frac{\partial g_{1,2}}{\partial \alpha}+\frac{\partial g_{1,3}}{\partial \alpha}+\frac{\partial g_{2,3}}{\partial \alpha}\right)(\alpha, \gamma / 2, \gamma) \\
& =\frac{\cos (\alpha) \sin (\gamma)}{1-\cos ^{2}(\alpha) \cos ^{2}(\gamma / 2)}-2\left(\frac{\sin (\gamma / 2)}{1-\cos ^{2}(\alpha) \cos ^{2}(\gamma / 2)}\right) \\
& =-\frac{2 \sin (\gamma / 2)}{1+\cos (\alpha) \cos (\gamma / 2)} .
\end{aligned}
$$

Hence the function $f_{1}(\alpha, \gamma / 2, \gamma, d)$ is monotonely increasing in $\alpha$. In view of (4.2.6) we obtain for $\alpha \in\left[\alpha_{0}, \pi / 2\right], \beta \in[0,2 \pi]$ and $\gamma \in[0, \pi / 2]$ :

$$
f_{1}(\alpha, \beta, \gamma, d) \geq f_{1}\left(\alpha_{0}, \gamma / 2, \gamma, d\right)
$$

Obviously, if $(\alpha, \beta, \gamma)$ satisfies (4.2.2) then also $\left(\alpha_{0}, \beta, \gamma\right)$. From the discussed behavior of the function $f_{1}(\alpha, \beta, \gamma, d)$ with respect to the parameters $\beta$ and $\gamma$ we see that $f_{1}\left(\alpha_{0}, \gamma / 2, \gamma\right)$ is monotonely increasing in $\gamma$. Together with (4.2.7) this shows the assertion. 


\subsection{Perturbing points}

Now we return to the 2-dimensional set $U(\phi)$ from definition 3.1.1. As in the last section let $\gamma$ be the angle between $y^{1}$ and $y^{2}$ and let $\alpha \in[0, \pi / 2]$ be the maximal angle of a vector of the configuration with the 2-dimensional plane $L$ (cf. (4.2.1)). For $\delta \in[0, \gamma]$ let $w_{\delta}$ be the point of the boundary of $U(\phi)$ with $\left\langle w_{\delta} /\left|w_{\delta}\right|, y^{1}\right\rangle=\cos (\delta)$. Then $U(\phi)=\left\{\lambda w_{\delta}: \lambda \in[0,1], \delta \in[0, \gamma]\right\}$ and by the definition of $D_{1}^{1}$ (cf. definition 3.1.2) we have

$$
V\left(D_{1}^{1}\right)=\int_{0}^{\gamma} \int_{0}^{\left|w_{\delta}\right|} r \cdot V\left(\left(r \frac{w_{\delta}}{\left|w_{\delta}\right|}+L^{\perp}\right) \cap D_{1}\right) d r d \delta .
$$

To evaluate the inner integral we use polar coordinates for the set $\left(r \frac{w_{\delta}}{\left|w_{\delta}\right|}+L^{\perp}\right) \cap D_{1}$ and obtain

$$
V\left(D_{1}^{1}\right)=\frac{1}{d-2} \int_{0}^{\gamma} \int_{S^{d-1} \cap L^{\perp}}\left|w_{\delta}\right|^{2} \int_{0}^{1} r \cdot h\left(r, w_{\delta}, z\right)^{d-2} d r d z d \delta,
$$

where for $r \in[0,1], \delta \in[0, \gamma]$ and $z \in S^{d-1} \cap L^{\perp}$

$$
h\left(r, w_{\delta}, z\right)=\max \left\{h \in \mathbb{R}^{\geq 0}: r w_{\delta}+h z \in D_{1}\right\},
$$

denotes the "height of $D_{1}$ " in direction $z$ over $r w_{\delta}$. For $\delta \in[0, \gamma]$ and $z \in S^{d-1} \cap L^{\perp}$ we are only interested in points $r w_{\delta}$ with a "height" in direction $z$ not less than 1. Hence we set

$$
r_{\delta, z}=\max \left\{r \in \mathbb{R}^{\geq 0}: h\left(r, w_{\delta}, z\right) \geq 1, r \leq 1\right\} .
$$

With this notation we get

$$
V\left(D_{1}^{1}\right) \geq \frac{1}{d-2} \int_{0}^{\gamma} \int_{S^{d-1} \cap L^{\perp}}\left|w_{\delta}\right|^{2} \int_{0}^{r_{\delta, z}} r \cdot h\left(r, w_{\delta}, z\right)^{d-2} d r d z d \delta .
$$

In general we can not assume that $\operatorname{conv}\left\{0, w_{\delta}\right\}+z \subset H$, i.e. $r_{\delta, z}=1$, because there might be a hyperplane $M_{j}=\left\{x \in E^{d}:\left\langle x^{j}, x\right\rangle=\left|x^{j}\right|^{2} / 2\right\}$, which separates a part of the set $\operatorname{conv}\left\{0, w_{\delta}\right\}+z$ from $H$, i.e.

$$
\left\langle x^{j}, r w_{\delta}+z\right\rangle>\frac{\left|x^{j}\right|^{2}}{2}, \quad r>r_{\delta, z} .
$$

But beside this negative influence, such a perturbing point $x^{j}$ has also a positive effect: For sufficiently small values of $r$ we find $r w_{\delta}+\epsilon_{r} z \in \operatorname{conv}\left(B^{d} \cup x^{j}+B^{d}\right) \cap H$ for suitable numbers $\epsilon_{r}>1$. Hence $h\left(r, w_{\delta}, z\right)>1$ for small $r$ and on account of the exponent $(d-2)$ in (4.3.1) the inner integral becomes large. Figure 4.3.1 shows this situation where the shaded region belongs to $D_{1}$ if there are no further perturbing points.

In the following we discuss the relationship between perturbing points and the size of the integral $\int_{0}^{r_{\delta, z}} r \cdot h\left(r, w_{\delta}, z\right)^{d-2} d r$ for a fixed pair of points $w_{\delta}, z$. The main result is: 


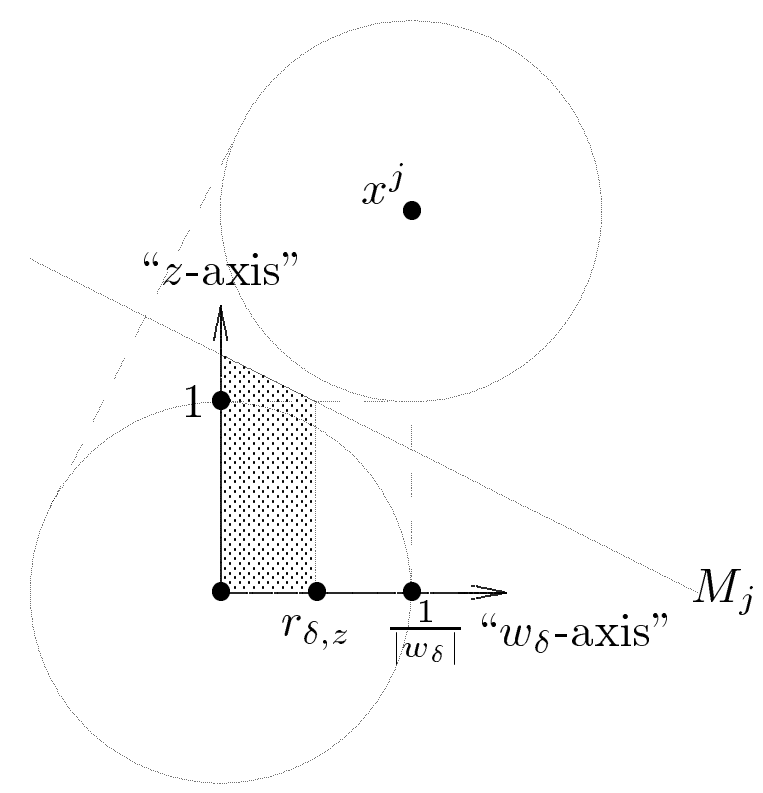

Figure 4.3.1.

Theorem 4.3.1 Let $d \geq 45, \delta \in[0, \gamma]$ and $z \in S^{d-1} \cap L^{\perp}$. Then

$$
\begin{aligned}
& \int_{0}^{r_{\delta, z}} r h\left(r, w_{\delta}, z\right)^{d-2} d r \geq f_{2}(\alpha, d) \\
= & \left\{\begin{array}{l}
1 / 2 \\
\min \left\{1 / 2, \int_{0}^{\frac{1-\sin (\alpha)}{\cos (\alpha)}} r\left(-r \frac{\cos (\alpha)}{\sin (\alpha)}+\frac{1}{\sin (\alpha)}\right)^{d-2} d r\right\}^{: \alpha<\pi / 4,}: \pi / 4 \leq \alpha \leq(7 / 20) \pi .
\end{array}\right.
\end{aligned}
$$

Let us remark, that the theorem is also true for dimensions less than 45. But since the crucial dimension is 45 , as we will see in the proof of theorem 45 , we state all theorems and lemmas with the bound 45 . As an immediate consequence of theorem 4.3.1 we obtain:

Corollary 4.3.1 Let $d \geq 45, \alpha \in[0,(7 / 20) \pi]$ and $\phi \in[0, \pi / 2]$. Then

$$
V\left(D_{1}^{1}\right) \geq V(U(\phi)) \kappa_{d-2} \cdot 2 \cdot f_{2}(\alpha, d) .
$$

Proof. $\quad$ By (4.3.1) and theorem 4.3.1 we have

$$
V\left(D_{1}^{1}\right) \geq \frac{1}{d-2} \int_{0}^{\gamma} \int_{S^{d-1} \cap L^{\perp}}\left|w_{\delta}\right|^{2} f_{2}(\alpha, d) d z d \delta=\left(\int_{0}^{\gamma} \frac{\left|w_{\delta}\right|^{2}}{2} d \delta\right) \kappa_{d-2} \cdot 2 \cdot f_{2}(\alpha, d) .
$$


At the end of this section we show that a similar result holds for the volume of the set $D_{1}^{2}$, but with a function depending on $\phi$ instead of $\alpha$.

The proof of theorem 4.3.1 is prepared by two lemmas for which we need the following functions:

Definition 4.3.1 For $\alpha \in[0, \pi / 2)$ and $0 \leq \zeta \leq \min \{2 \sin (\alpha), 2 \cos (\alpha)\}$ let

$$
\begin{aligned}
& \mu(\alpha, \zeta)=\left(\sqrt{4-\zeta^{2}}-2 \sin (\alpha)\right) /(2+\zeta-2 \sin (\alpha)), \\
& g_{1}(\alpha, \zeta, d)=\int_{0}^{\mu(\alpha, \zeta)} r\left(r \frac{\zeta}{\sqrt{4-\zeta^{2}}}+\frac{2}{\sqrt{4-\zeta^{2}}}\right)^{d-2} d r \\
& g_{2}(\alpha, \zeta, d)=\int_{\mu(\alpha, \zeta)}^{\sqrt{(2-\zeta) /(2+\zeta)}} r\left(r \frac{\sin (\alpha)-1}{\sin (\alpha)} \sqrt{\frac{2+\zeta}{2-\zeta}}+\frac{1}{\sin (\alpha)}\right)^{d-2} d r \\
& g_{3}(\alpha, \zeta, d)=g_{1}(\alpha, \zeta, d)+g_{2}(\alpha, \zeta, d), \\
& g(\alpha, d)=\min \left\{g_{3}(\alpha, \zeta, d): 0 \leq \zeta \leq \min \{2 \sin (\alpha), 2 \cos (\alpha)\}\right\},
\end{aligned}
$$

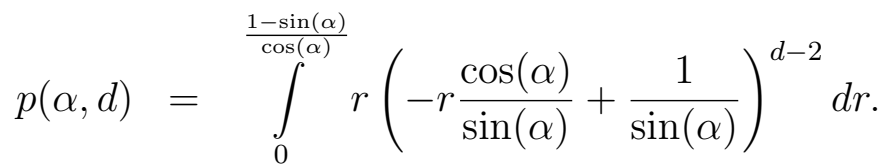

We note that $g_{3}(\alpha, \zeta, d)$ is a continuous function for $\alpha \in[0, \pi / 2)$ and $0 \leq \zeta \leq$ $\min \{2 \sin (\alpha), 2 \cos (\alpha)\}$ with $g_{3}(\alpha, 0, d)=g_{1}(\alpha, 0, d)=1 / 2, \alpha \in[0, \pi / 2)$.

Lemma 4.3.1 Let $\alpha \in[0, \pi / 2), \delta \in[0, \gamma]$ and $z \in S^{d-1} \cap L^{\perp}$. Then

$$
\int_{0}^{r_{\delta, z}} r h\left(r, w_{\delta}, z\right)^{d-2} \geq \begin{cases}g(\alpha, d) & : \alpha<\pi / 4 \\ \min \{g(\alpha, d), p(\alpha, d)\}: \pi / 4 \leq \alpha\end{cases}
$$

Proof. Instead of $w_{\delta}$ we write $w$ for short. For the proof we replace the DirichletVoronoï cell $H$ by the "smaller" set $H_{s} \subset H$ given by

$$
H_{s}=\left\{x \in E^{d}:\left\langle x, y^{j}\right\rangle \leq 1,1 \leq j \leq n-1\right\}
$$

and define analogously to $h\left(r, w_{\delta}, z\right), r_{\delta, z}$ :

$$
\begin{aligned}
h_{s}(r) & =\max \left\{h \in \mathbb{R}^{\geq 0}: r w+h z \in H_{s} \cap\left(\operatorname{conv}(C)+B^{d}\right)\right\}, \\
r_{s} & =\max \left\{r \in \mathbb{R}^{\geq 0}: h_{s}(r) \geq 1, r \leq 1\right\} .
\end{aligned}
$$


As $h_{s}(r) \leq h(r, w, z)$ and $r_{s} \leq r_{\delta, z}$ it suffices to show

$$
\int_{0}^{r_{s}} r h_{s}(r)^{d-2} \geq \begin{cases}g(\alpha, d) & : \alpha<\pi / 4 \\ \min \{g(\alpha, d), p(\alpha, d)\} & : \pi / 4 \leq \alpha .\end{cases}
$$

Observe that $B^{d} \subset H_{s}$ and thus $w \in\left(\operatorname{conv}(C)+B^{d}\right) \cap H_{s}$. In the case $r_{s}=1$ there is nothing to prove because $\int_{0}^{1} r h_{s}(r)^{d-2} d r \geq 1 / 2$ and $g(\alpha, 0, d)=1 / 2$. So we may assume $r_{s}<1$. Hence there exists a point $u \in\left\{2 y^{1}, \ldots, 2 y^{n-1}\right\}$ with

$$
\left\langle u, r_{s} w+z\right\rangle=2
$$

Let

$$
u=\sigma v+\tau \frac{w}{|w|}+\zeta z
$$

with $\sigma, \tau, \zeta \in \mathbb{R}$ and $v \in \operatorname{lin}(w, z)^{\perp},|v|=1$. Then

$$
\sigma^{2}+\tau^{2}+\zeta^{2}=4
$$

and (4.3.3) is equivalent to

$$
\tau|w| r_{s}+\zeta=2
$$

Obviously, we have $0 \leq \tau, \zeta \leq 2$. We claim that

$$
\zeta \leq 2 \sin (\alpha)
$$

By the definition of $\alpha$ we get $\left\langle y^{j}, x\right\rangle \leq \sin (\alpha)$ for all $x \in S^{d-1} \cap L^{\perp}$ and $1 \leq j \leq n$. Since $r_{s}<1$ we have $\alpha>0$ and thus

$$
(1 / \sin (\alpha)) x \in H_{s}, \quad x \in S^{d-1} \cap L^{\perp} .
$$

As $(2 / \zeta) z \notin \operatorname{int}\left(H_{s}\right)$ it follows $2 / \zeta \geq 1 / \sin (\alpha)$.

In particular (4.3.6) and (4.3.5) imply $\tau>0$ and we may write

$$
r_{s}=\frac{2-\zeta}{|w| \tau} .
$$

Moreover, by (4.3.7) and lemma 3.1.1 (with $H_{s}$ instead of $H$ ) we obtain the lower bound

$$
|w| r_{s} \geq c_{1}(1,1 / \sin (\alpha)-1)=(1-\sin (\alpha)) / \cos (\alpha) .
$$

Now we study the positive effects of such a perturbing point $u$. For $r \in[0,1]$ let

$$
h^{\prime}(r)=\max \left\{h \in \mathbb{R}^{\geq 0}: r w+h z \in \operatorname{conv}\{0, u\}+B^{d}\right\} .
$$

The function $h^{\prime}(r)$ can easily be determined by the equality

$$
\left|r w+h^{\prime}(r) z-\frac{\left\langle r w+h^{\prime}(r) z, u / 2\right\rangle}{2} u\right|^{2}=1,
$$


which says that the point given by the orthogonal projection of $r w+h^{\prime}(r) z$ onto the hyperplane with normal vector $u$ has unit length. We obtain with (4.3.4):

$$
\begin{aligned}
h^{\prime}(r) & =\frac{|w| r \tau \zeta+2 \sqrt{4-\zeta^{2}+\left(-4+\tau^{2}+\zeta^{2}\right)|w|^{2} r^{2}}}{4-\zeta^{2}} \\
& =\frac{|w| r \tau \zeta+2 \sqrt{4-\zeta^{2}-\sigma^{2}|w|^{2} r^{2}}}{4-\zeta^{2}} .
\end{aligned}
$$

We distinguish two cases.

i) $1 / \sin (\alpha) \leq h^{\prime}(0)=2 / \sqrt{4-\zeta^{2}}$.

Then $\sin (\alpha) \geq\left(1-(\zeta / 2)^{2}\right)^{1 / 2}$ and by (4.3.6) we get $\sin (\alpha) \geq \cos (\alpha)$. Hence $\alpha \geq \pi / 4$. Furthermore, since $h^{\prime}(0) z \in \operatorname{conv}(C)+B^{d}$ we may deduce from (4.3.7) that $(1 / \sin (\alpha)) z \in\left(\operatorname{conv}(C)+B^{d}\right) \cap H_{s}$. Together with (4.3.9) it follows

$$
\operatorname{conv}\left\{0, \frac{1}{\sin (\alpha)} z, \frac{1-\sin (\alpha)}{\cos (\alpha)|w|} w, \frac{1-\sin (\alpha)}{\cos (\alpha)|w|} w+z\right\} \subset\left(\operatorname{conv}(C)+B^{d}\right) \cap H_{s} .
$$

So

$$
h_{s}(r) \geq \frac{1}{\sin (\alpha)}-r \frac{|w| \cos (\alpha)}{\sin (\alpha)}, \quad \text { for } r \in\left[0, \frac{1-\sin (\alpha)}{|w| \cos (\alpha)}\right]
$$

As $|w| \leq 1$ we have

$$
\int_{0}^{r_{s}} r h_{s}(r)^{d-2} d r \geq p(\alpha, d) \text { for } \alpha \geq \pi / 4
$$

ii) $1 / \sin (\alpha) \geq h^{\prime}(0)=2 / \sqrt{4-\zeta^{2}}$.

Then $4 \sin ^{2}(\alpha) \leq 4-\zeta^{2}$ which implies $\zeta \leq 2 \cos (\alpha)$ and together with (4.3.6)

$$
\zeta \leq \min \{2 \sin (\alpha), 2 \cos (\alpha)\}
$$

Now we determine the smallest value of $r_{0}$ such that the point $r_{0} w+h^{\prime}\left(r_{0}\right) z$ lies in the hyperplane $M=\left\{x \in E^{d}:\langle u, x\rangle=2\right\}$. Such a pair $\left(r_{0}, h^{\prime}\left(r_{0}\right)\right)$ (if it exists) must satisfy the relations:

$$
r_{0}|w| \tau+h^{\prime}\left(r_{0}\right) \zeta=2, \quad r_{0}^{2}|w|^{2}+h^{\prime}\left(r_{0}\right)^{2}=2 .
$$

The first equation means that the point lies in the hyperplane $M$ and the second one expresses the property that $r_{0} w+h^{\prime}\left(r_{0}\right) z$ belongs to the boundary of the $(d-1)$-dimensional unit ball with center $u / 2$ embedded in $M$. By (4.3.12) we find

$$
r_{0}^{2}|w|^{2}+\left(\frac{2-r_{0}|w| \tau}{\zeta}\right)^{2}=2
$$


and so

$$
r_{0}=\frac{2 \tau-\zeta \sqrt{2\left(\tau^{2}+\zeta^{2}\right)-4}}{|w|\left(\tau^{2}+\zeta^{2}\right)} .
$$

We note that $r_{0}$ is well-defined, i.e. $\tau^{2}+\zeta^{2} \geq 2$ : Since $r_{s},|w| \leq 1$ we have $\tau+\zeta \geq 2$ (cf.(4.3.5)) and thus $\tau^{2}+\zeta^{2} \geq 2$. Moreover, from (4.3.11) we get $\zeta \leq \sqrt{2}$ which implies $r_{0} \geq 0$. We also have $r_{0} \leq r_{s}$. To show this we use (4.3.8) and obtain

$$
\begin{aligned}
r_{0} \leq r_{s} & \Leftrightarrow \frac{2 \tau-\zeta \sqrt{2\left(\tau^{2}+\zeta^{2}\right)-4}}{|w|\left(\tau^{2}+\zeta^{2}\right)} \leq \frac{2-\zeta}{|w| \tau} \\
& \Leftrightarrow-\tau \zeta \sqrt{2\left(\tau^{2}+\zeta^{2}\right)-4} \leq \zeta\left(2 \zeta-\tau^{2}-\zeta^{2}\right) \\
& \Leftrightarrow \tau^{2}+\zeta^{2} \leq 2 \zeta+\tau \sqrt{2\left(\tau^{2}+\zeta^{2}\right)-4} .
\end{aligned}
$$

Let $h(\tau, \zeta)=\tau^{2}+\zeta^{2}-2 \zeta-\tau \sqrt{2\left(\tau^{2}+\zeta^{2}\right)-4}$. In order to show $h(\tau, \zeta) \leq 0$ for $0 \leq \zeta \leq \sqrt{2}$ and $\tau \in\left[2-\zeta, \sqrt{4-\zeta^{2}}\right]$ we calculate the first partial derivative of $h$ with respect to $\tau$ :

$$
\frac{\partial h(\tau, \zeta)}{\partial \tau}=\frac{2 \tau \sqrt{2\left(\tau^{2}+\zeta^{2}\right)-4}-4 \tau^{2}-2 \zeta^{2}+4}{\sqrt{2\left(\tau^{2}+\zeta^{2}\right)-4}} .
$$

From this we deduce

$$
\begin{aligned}
\frac{\partial h(\tau, \zeta)}{\partial \tau} \leq 0 & \Leftrightarrow \tau \sqrt{2\left(\tau^{2}+\zeta^{2}\right)-4} \leq 2 \tau^{2}+\zeta^{2}-2 \\
& \Leftrightarrow \tau^{2}\left(\frac{\zeta^{2}-2}{2 \tau^{2}+\zeta^{2}-2}+1\right) \leq 2 \tau^{2}+\zeta^{2}-2
\end{aligned}
$$

Since $\zeta \leq \sqrt{2}$ and $\tau^{2}+\zeta^{2} \geq 2$ the function $h(\tau, \zeta)$ is monotonely decreasing in $\tau$. Thus $h(\tau, \zeta) \leq h(2-\zeta, \zeta)=2(2-\zeta)\left((1-\zeta)-\sqrt{(1-\zeta)^{2}}\right) \leq 0$. Hence $r_{0} \leq r_{s}$.

From the right hand side equation in (4.3.12) it follows $h^{\prime}\left(r_{0}\right)>1$ and substituting $r_{0}$ from (4.3.13) in the left hand side equation of (4.3.12) yields

$$
h^{\prime}\left(r_{0}\right)=\frac{2 \zeta+\tau \sqrt{2\left(\tau^{2}+\zeta^{2}\right)-4}}{\tau^{2}+\zeta^{2}} .
$$

Now let

$$
\begin{aligned}
S_{1} & =\operatorname{conv}\left\{0, h^{\prime}(0) z, r_{0} w, r_{0} w+h^{\prime}\left(r_{0}\right) z\right\}, \\
S_{2} & =\operatorname{conv}\left\{r_{0} w, r_{0} w+h^{\prime}\left(r_{0}\right) z, r_{s} w, r_{s} w+z\right\} \\
T(\alpha) & =\operatorname{conv}\left\{0,(1 / \sin (\alpha)) z, r_{s} w, r_{s} w+z\right\} .
\end{aligned}
$$


Clearly, $S_{1}, S_{2} \subset \operatorname{conv}(C)+B^{d}$ and from the definition of $r_{s}$ and (4.3.7) we have $T(\alpha) \subset H_{s}$. Hence

$$
T(\alpha) \cap\left(S_{1} \cup S_{2}\right) \subset\left(\operatorname{conv}(C)+B^{d}\right) \cap H_{s}
$$

In the following we derive from the set $T(\alpha) \cap\left(S_{1} \cup S_{2}\right)$ a lower bound for the function $h_{s}(r)$. To this end we first show that we may assume $\tau^{2}+\zeta^{2}=4$. Let

$$
\tau_{1}=r_{0}|w|+h^{\prime}\left(r_{0}\right) \text { and } \zeta_{1}=h^{\prime}\left(r_{0}\right)-r_{0}|w|
$$

Then on account of (4.3.12), $r_{0},|w| \leq 1$ and $h^{\prime}\left(r_{0}\right)>1$ we have

$$
\tau_{1}, \zeta_{1}>0, \quad \tau_{1}^{2}+\zeta_{1}^{2}=4 \text { and } \tau_{1} r_{0}|w|+\zeta_{1} h^{\prime}\left(r_{0}\right)=2 .
$$

Now let $\tilde{u}=\tau_{1} w /|w|+\zeta_{1} z$ and let $\tilde{r}_{s}, \tilde{h}^{\prime}(r), \tilde{r}_{0}, \tilde{S}_{1}, \tilde{S}_{2}, \tilde{T}(\alpha)$ be defined as above for the point $u$. By the choice of $\tau_{1}, \zeta_{1}$ we get $\tilde{r}_{0}=r_{0}=\left(\tau_{1}-\zeta_{1}\right) /(2|w|)$ and $\tilde{h}^{\prime}\left(\tilde{r}_{0}\right)=$ $h^{\prime}\left(r_{0}\right)=\left(\tau_{1}+\zeta_{1}\right) / 2(\operatorname{cf} .(4.3 .13),(4.3 .14))$. Furthermore, as $\tau r_{0}|w|+\zeta h^{\prime}\left(r_{0}\right)=2$ and $\tau^{2}+\zeta^{2} \leq 4$ we obtain $\tau_{1} \geq \tau, \zeta_{1} \leq \zeta$ and (cf. (4.3.8)):

$$
\tilde{h}^{\prime}(0)=\frac{2}{\tau_{1}} \leq \frac{2}{\sqrt{4-\zeta^{2}}}=h^{\prime}(0), \quad \tilde{r}_{s}=\frac{2-\zeta_{1}}{|w| \tau_{1}} \leq \frac{2-\zeta}{|w| \tau}=r_{s} .
$$

Hence we have $\tilde{S}_{1} \subset S_{1}, \tilde{S}_{2} \subset S_{2}$ and $\tilde{T}(\alpha) \subset T(\alpha)$. Figure 4.3 .2 shows the set $S_{1} \cup S_{2}$ and the new set $\tilde{S}_{1} \cup \tilde{S}_{2}$.

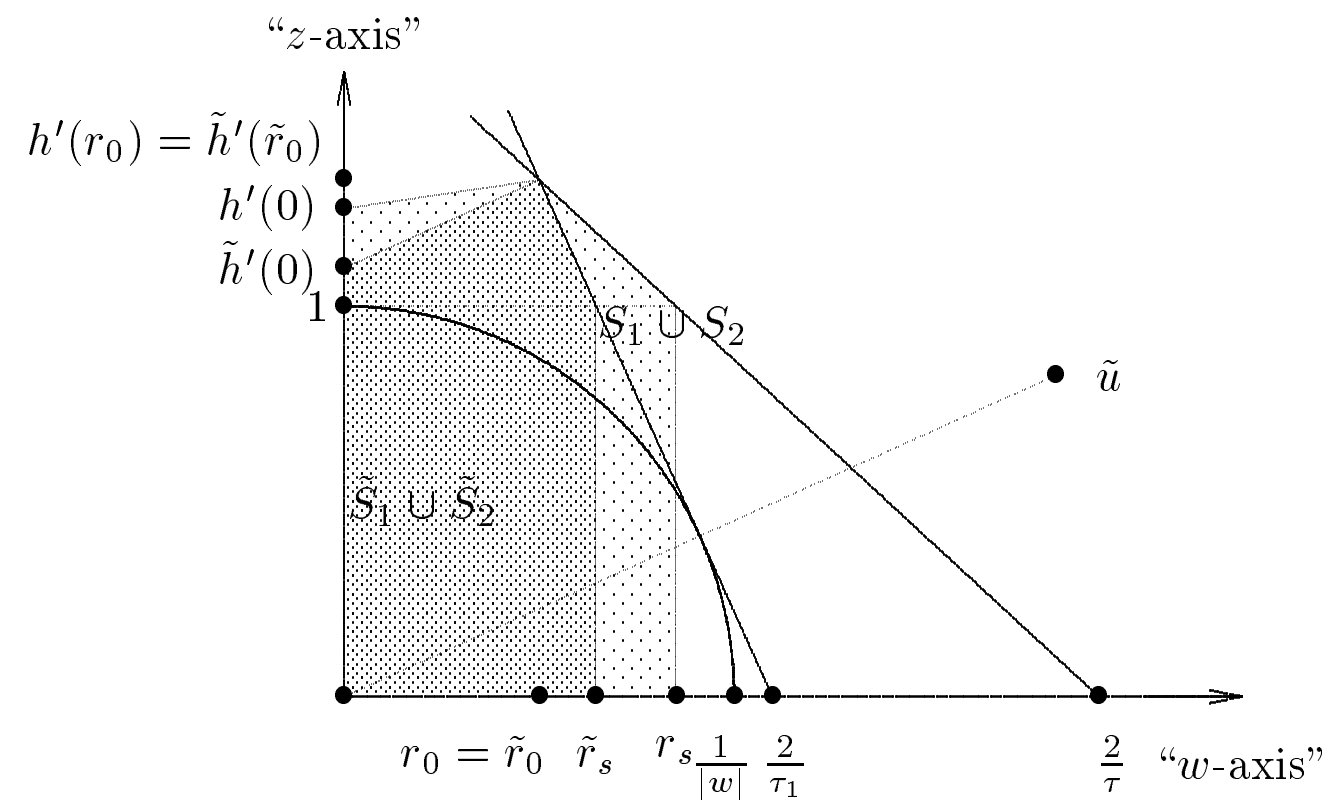

Figure 4.3.2. 
So the sets $S_{1}, S_{2}, T(\alpha)$ becomes "minimal" (with respect to inclusion) for parameters $\tau, \zeta \geq 0$ which satisfy $\tau^{2}+\zeta^{2}=4$ and $\zeta \leq \min \{2 \sin (\alpha), 2 \cos (\alpha)\}$ (cf. (4.3.11)). Therefore, in the sequel we assume $\tau^{2}+\zeta^{2}=4$ and thus (cf. (4.3.8), (4.3.13), (4.3.14)):

$$
\begin{aligned}
r_{s} & =\frac{\sqrt{2-\zeta}}{\sqrt{2+\zeta}|w|}, \quad r_{0}=\frac{\sqrt{4-\zeta^{2}}-\zeta}{2|w|} \\
h^{\prime}(0) & =\frac{2}{\sqrt{4-\zeta^{2}}}, \quad h^{\prime}\left(r_{0}\right)=\frac{\sqrt{4-\zeta^{2}}+\zeta}{2} .
\end{aligned}
$$

Next we determine the intersection $T(\alpha) \cap\left(S_{1} \cup S_{2}\right)$. Let $\chi_{1} w+\chi_{2} z$ be the point of intersection of the two segments $\operatorname{conv}\left\{(1 / \sin (\alpha)) z, r_{s} w+z\right\}$ and $\operatorname{conv}\left\{h^{\prime}(0) z, r_{0} w+h^{\prime}\left(r_{0}\right) w\right\}$ (see figure 4.3.3).

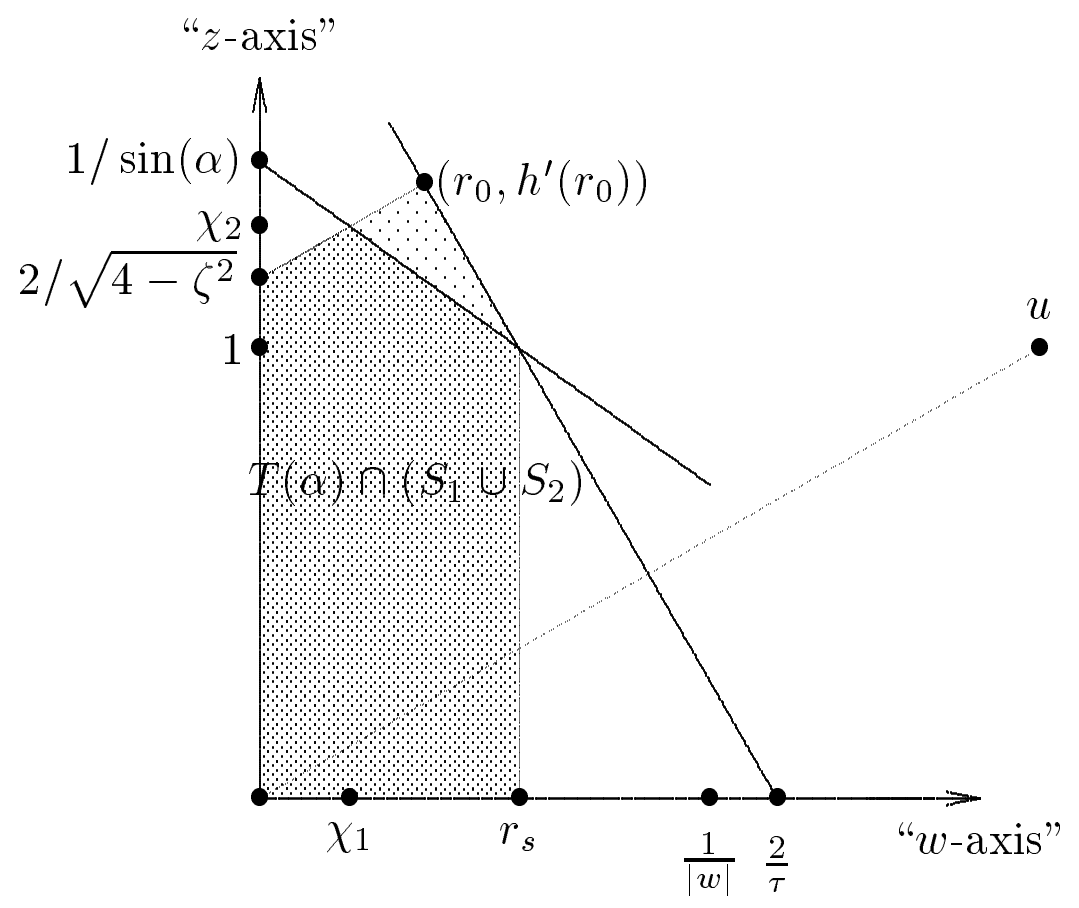

Figure 4.3.3.

Observe that on account of $h^{\prime}(0) \leq 1 / \sin (\alpha) \leq 2 / \zeta$ such a point exists. Then we obviously have

$$
\begin{aligned}
T(\alpha) \cap\left(S_{1} \cup S_{2}\right) & =\operatorname{conv}\left\{0, h^{\prime}(0) z, \chi_{1} w, \chi_{1} w+\chi_{2} z\right\} \\
\cup & \operatorname{conv}\left\{\chi_{1} w, \chi_{1} w+\chi_{2} z, r_{s} w, r_{s} w+z\right\}
\end{aligned}
$$

and for $\chi_{1}, \chi_{2}$ we find (cf. (4.3.16)): 


$$
\begin{aligned}
\chi_{1} & =\mu(\alpha, \zeta) /|w| \\
\chi_{2} & =\frac{2}{\sqrt{4-\zeta^{2}}}+\mu(\alpha, \zeta) \frac{\zeta}{\sqrt{4-\zeta^{2}}} \\
& =\frac{1}{\sin (\alpha)}+\mu(\alpha, \zeta) \frac{\sqrt{2+\zeta}}{\sqrt{2-\zeta}} \frac{\sin (\alpha)-1}{\sin (\alpha)} .
\end{aligned}
$$

Hence

$$
\begin{aligned}
& h_{s}(r) \geq \frac{2}{\sqrt{4-\zeta^{2}}}+r|w| \frac{\zeta}{\sqrt{4-\zeta^{2}}} \text { for } \quad 0 \leq r \leq \frac{\mu(\alpha, \zeta)}{|w|} \text { and } \\
& h_{s}(r) \geq \frac{1}{\sin (\alpha)}+r|w| \frac{\sqrt{2+\zeta}}{\sqrt{2-\zeta}} \frac{\sin (\alpha)-1}{\sin (\alpha)} \text { for } \quad \frac{\mu(\alpha, \zeta)}{|w|} \leq r \leq \frac{\sqrt{2-\zeta}}{\sqrt{2+\zeta}|w|} .
\end{aligned}
$$

Together with $|w| \leq 1$ and the first case (4.3.10) this shows (4.3.2).

In the next lemma we give a lower bound for the function $g(\alpha, d)$ depending on the dimension and $\alpha$.

Lemma 4.3.2 Let $d \geq 45$. Then

$$
g(\alpha, d) \begin{cases}=\frac{1}{2} & : \alpha \leq \pi / 4 \\ \geq \min \left\{\frac{1}{2}, p(\alpha, d)\right\} & : \pi / 4 \leq \alpha \leq(7 / 20) \pi\end{cases}
$$

Proof. First we consider the behavior of $g_{3}(\alpha, \zeta, d)$ with respect to $\alpha$. For a given $\zeta$ the set $T(\alpha)$ in (4.3.15) becomes "smaller" (with respect to inclusion) if we increase the angle $\alpha$. So, by construction, the function $g_{3}(\alpha, \zeta, d)$ is monotonely decreasing in $\alpha$. On account of $\zeta \leq \min \{2 \sin (\alpha), 2 \cos (\alpha)\}$ this means that

$$
g(\alpha, d) \geq \min \left\{g_{3}(\pi / 4, \zeta, d): 0 \leq \zeta \leq \sqrt{2}\right\}, \quad \alpha \leq \pi / 4,
$$

and for $\alpha \geq \pi / 4$ :

$$
\begin{aligned}
g(\alpha, d) \geq \min \{ & g_{3}(\alpha, 2 \cos (\alpha), d), \\
& \left.\min \left\{g_{3}((7 / 20) \pi, \zeta, d): 0 \leq \zeta \leq 2 \cos ((7 / 20) \pi)\right\}\right\} .
\end{aligned}
$$

For $\alpha \geq \pi / 4$ we have

$$
\begin{aligned}
g_{3}(\alpha, 2 \cos (\alpha), d) & =g_{2}(\alpha, 2 \cos (\alpha), d) \\
& =\left(\frac{\cos (\alpha)}{1-\sin (\alpha)} \sqrt{\frac{1-\cos (\alpha)}{1+\cos (\alpha)}}\right)^{2} p(\alpha, d) \geq p(\alpha, d),
\end{aligned}
$$

where we use the substitution $r=\cos (\alpha) /(1-\sin (\alpha)) \cdot(1-\cos (\alpha)) /(1+\cos (\alpha))^{1 / 2} t$. So, as $g(\alpha, d) \leq g_{3}(\alpha, 0, d)=1 / 2$ and $g_{3}$ increases in $d$ it suffices to prove 


$$
\begin{aligned}
\min \left\{g_{3}(\pi / 4, \zeta, 45): 0 \leq \zeta \leq \sqrt{2}\right\} & =1 / 2, \\
\min \left\{g_{3}((7 / 20) \pi, \zeta, 45): 0 \leq \zeta \leq 2 \cos ((7 / 20) \pi)\right\} & =1 / 2
\end{aligned}
$$

Figure 4.3.4 shows a plot of the functions $\log _{2}\left(g_{3}(\pi / 4, \zeta, 45)\right), \zeta \in[0, \sqrt{2}]$ and $\log _{2}\left(g_{3}((7 / 20) \pi, \zeta, 45)\right), \zeta \in[0,2 \cos ((7 / 20) \pi)]$. The plots were generated by the program Mathematica ${ }^{2}$.
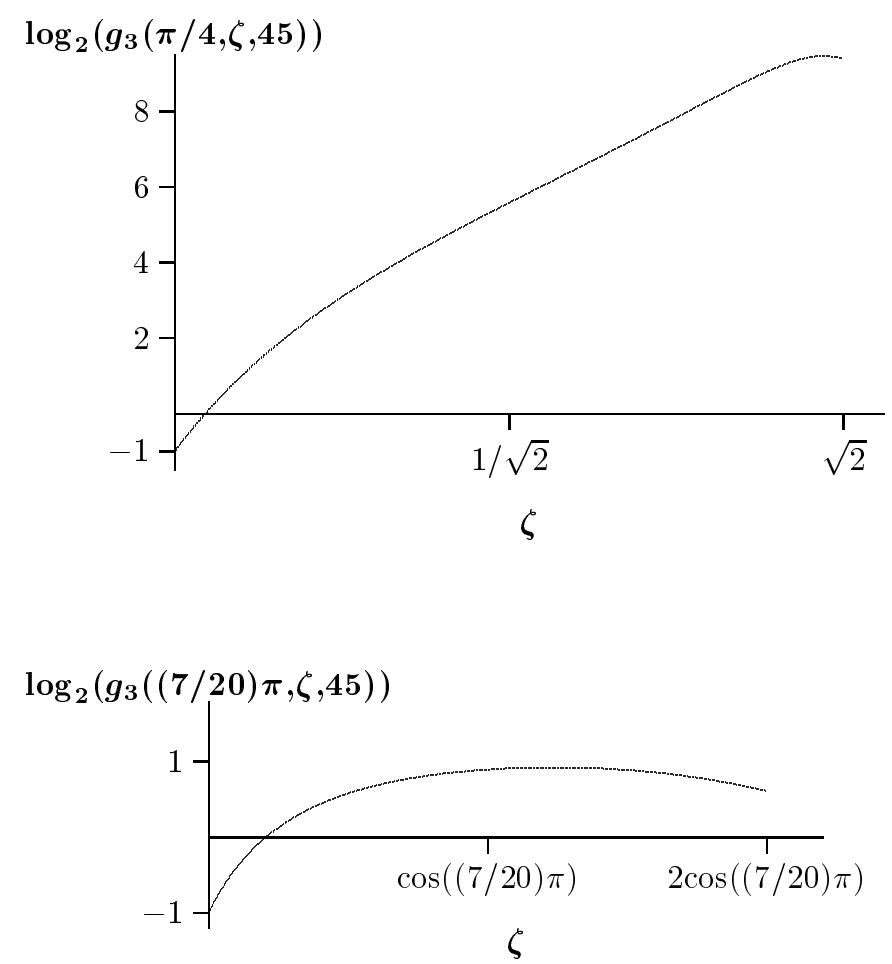

Figure 4.3.4.

We "see" that (4.3.18) holds. But beside this more visual proof we give in the following for a fixed $\alpha$ a lower bound of $g_{3}(\alpha, \zeta, 45)$ from which (4.3.18) follows by evaluating a certain auxiliary function at finitely many points. To this end we write for abbreviation m.s. or m.d. for monotonely increasing or monotonely decreasing, respectively. We recall (cf. definition 4.3.1):

$$
g_{1}(\alpha, \zeta, d)=\int_{0}^{\mu(\alpha, \zeta)} r\left(r \frac{\zeta}{\sqrt{4-\zeta^{2}}}+\frac{2}{\sqrt{4-\zeta^{2}}}\right)^{d-2} d r .
$$

\footnotetext{
${ }^{2}$ C $1988,1991,1992$ von Wolfram Research Inc.
} 
Substituting $r=\mu(\alpha, \zeta) \cdot t$ yields

$g_{1}(\alpha, \zeta, d)=\mu(\alpha, \zeta)^{2} \int_{0}^{1} t \omega(t, \zeta, \alpha)^{d-2} d t, \quad$ with $\omega(t, \zeta, \alpha)=t \frac{\mu(\alpha, \zeta) \zeta}{\sqrt{4-\zeta^{2}}}+\frac{2}{\sqrt{4-\zeta^{2}}}$

Obviously, $\mu(\alpha, \zeta)$ is m.d. in $\zeta$. We claim that for $t \in[0,1]$ :

$$
\omega(t, \zeta, \alpha) \text { is m.i. in } \zeta .
$$

Since $2 / \sqrt{4-\zeta^{2}}$ is $\mathrm{m} . \mathrm{i}$. in $\zeta$ it suffices to prove that $\omega(1, \zeta, \alpha)$ is m.i. By $(4.3 .17)$ we have

$$
\omega(1, \zeta, \alpha)=1+\frac{\sin (\alpha)-1}{\sin (\alpha)} \frac{\sqrt{2+\zeta}}{\sqrt{2-\zeta}}\left(\mu(\alpha, \zeta)-\frac{\sqrt{2-\zeta}}{\sqrt{2+\zeta}}\right) .
$$

Now $\mu(\alpha, \zeta)-((2-\zeta) /(2+\zeta))^{1 / 2} \leq 0$ and thus it even suffices to show that $\mu(\alpha, \zeta)-((2-\zeta) /(2+\zeta))^{1 / 2}$ is m.d. for $0 \leq \zeta \leq \min \{2 \sin (\alpha), 2 \cos (\alpha)\}$. This can be easily verified by calculating the first derivative. Altogether we obtain for $\zeta \in\left[\zeta_{1}, \zeta_{2}\right]$ :

$$
g_{1}(\alpha, \zeta, d) \geq \mu\left(\alpha, \zeta_{2}\right)^{2} \int_{0}^{1} t \omega\left(t, \zeta_{1}, \alpha\right)^{d-2} d t
$$

For the function $g_{2}$ we use the substitution $r=\mu(\alpha, \zeta) t+(1-t)((2-\zeta) /(2+\zeta))^{1 / 2}$ and get (cf. (4.3.21)):

$$
\begin{aligned}
g_{2}(\alpha, \zeta, d)= & \left(\frac{\sqrt{2-\zeta}}{\sqrt{2+\zeta}}-\mu(\alpha, \zeta)\right) \times \\
& \int_{0}^{1}\left(\mu(\alpha, \zeta) t+(1-t) \frac{\sqrt{2-\zeta}}{\sqrt{2+\zeta}}\right)((\omega(1, \zeta, \alpha)-1) t+1)^{d-2} d t \\
\geq & \left(\frac{\sqrt{2-\zeta}}{\sqrt{2+\zeta}}-\mu(\alpha, \zeta)\right) \times \\
& \int_{0}^{1}\left((1-t) \frac{\sqrt{2-\zeta}}{\sqrt{2+\zeta}}\right)((\omega(1, \zeta, \alpha)-1) t+1)^{d-2} d t
\end{aligned}
$$

Since $((2-\zeta) /(2+\zeta))^{1 / 2}-\mu(\alpha, \zeta)$ is m.i. and $((2-\zeta) /(2+\zeta))^{1 / 2}$ is m.d. in $\zeta$ we find for $\zeta \in\left[\zeta_{1}, \zeta_{2}\right]$ (cf. $\left.(4.3 .20)\right)$ :

$$
\begin{aligned}
g_{2}(\alpha, \zeta, d) \geq & \left(\frac{\sqrt{2-\zeta_{1}}}{\sqrt{2+\zeta_{1}}}-\mu\left(\alpha, \zeta_{1}\right)\right) \frac{\sqrt{2-\zeta_{2}}}{\sqrt{2+\zeta_{2}}} \times \\
& \int_{0}^{1}(1-t)\left(\left(\omega\left(1, \zeta_{1}, \alpha\right)-1\right) t+1\right)^{d-2} d t .
\end{aligned}
$$


Let $u\left(\alpha, \zeta_{1}, \zeta_{2}, d\right)$ be the sum of the right hand sides in (4.3.22) and (4.3.23). For $\zeta \in\left[\zeta_{1}, \zeta_{2}\right]$ it follows:

$$
g_{3}(\alpha, \zeta, d) \geq u\left(\alpha, \zeta_{1}, \zeta_{2}, d\right) .
$$

Unfortunately, for $\zeta_{1}=0$ and $\zeta_{2}>0$ we have $u\left(\alpha, 0, \zeta_{2}, d\right)<1 / 2$. Hence for small values of $\zeta$ we need another estimate for the function $g_{3}$. By (4.3.19) we get

$$
\begin{aligned}
g_{3}(\alpha, \zeta, d) & \geq g_{1}(\alpha, \zeta, d) \\
& =\left(\frac{2}{\sqrt{4-\zeta^{2}}}\right)^{d-2} \mu(\alpha, \zeta)^{2} \int_{0}^{1} t\left(t \frac{\zeta}{2} \mu(\alpha, \zeta)+1\right)^{d-2} d t \\
& \geq\left(\frac{2}{\sqrt{4-\zeta^{2}}}\right)^{d-2} \mu(\alpha, \zeta)^{2} \frac{1}{2}\left(\frac{1}{2} \frac{\zeta}{2} \mu(\alpha, \zeta)+1\right)^{d-2},
\end{aligned}
$$

where the last inequality results from the convexity of the function $t(t \zeta \mu(\alpha, \zeta) / 2+$ 1). So, in order to prove $g_{3}(\alpha, \zeta, d) \geq 1 / 2$ it suffices to show

$$
\frac{2}{\sqrt{4-\zeta^{2}}} \mu(\alpha, \zeta)^{2 /(d-2)}\left(\frac{\zeta}{4} \mu(\alpha, \zeta)+1\right) \geq 1
$$

To this end let $\psi(\alpha, \zeta)$ be defined by

$$
\begin{aligned}
\mu(\alpha, \zeta) & =\frac{\sqrt{4-\zeta^{2}} / 2}{1+(\zeta / 2) \psi(\alpha, \zeta)}, \text { i.e. } \\
\psi(\alpha, \zeta) & =\frac{\sqrt{4-\zeta^{2}}+2 \sin (\alpha)\left(2-\sqrt{4-\zeta^{2}}\right) / \zeta}{\sqrt{4-\zeta^{2}}+2 \sin (\alpha)} .
\end{aligned}
$$

By the Bernoulli inequality $(1+x)^{m} \geq 1+m x$ for $x \geq-1, m \in \mathbb{N}$, we obtain

$$
\left(1+\frac{2}{d-2} \frac{\zeta}{2} \psi(\alpha, \zeta)\right)^{(d-2) / 2} \geq 1+\frac{\zeta}{2} \psi(\alpha, \zeta)=\frac{\sqrt{4-\zeta^{2}} / 2}{\mu(\alpha, \zeta)} .
$$

Hence

$$
\mu(\alpha, \zeta)^{2 /(d-2)} \geq \frac{\left(\sqrt{4-\zeta^{2}} / 2\right)^{2 /(d-2)}}{1+(2 /(d-2))(\zeta / 2) \psi(\alpha, \zeta)} \geq \frac{\sqrt{4-\zeta^{2}} / 2}{1+(2 /(d-2))(\zeta / 2) \psi(\alpha, \zeta)} .
$$

So (4.3.25) holds for all $\zeta$ with

$$
\mu(\alpha, \zeta) \geq \frac{4}{d-2} \psi(\alpha, \zeta)
$$

Calculating the first partial derivative with respect to $\zeta$ shows that $\psi(\alpha, \zeta)$ is m.i. in $\zeta, \zeta \leq \sqrt{2}$. As $\mu(\alpha, \zeta)$ is m.d. in $\zeta$ we have shown that for each $\zeta_{*}(\alpha)$ satisfying (4.3.26) and $\zeta \in\left[0, \zeta_{*}(\alpha)\right]$ one has

$$
g_{3}(\alpha, \zeta, d) \geq 1 / 2 \text {. }
$$


Finally, let $\zeta^{*}(\alpha)=\min \{2 \sin (\alpha), 2 \cos (\alpha)\}, m \in \mathbb{N}$ and $l(i)=\zeta_{*}(\alpha)+(i / m)\left(\zeta^{*}(\alpha)\right.$ $\left.-\zeta_{*}(\alpha)\right), 0 \leq i \leq m$. From (4.3.24) it follows

$$
g_{3}(\alpha, \zeta, d) \geq \min _{0 \leq i \leq m-1}\{u(\alpha, l(i), l(i+1), d)\} \quad \text { for } \zeta \in\left[\zeta_{*}(\alpha), \zeta^{*}(\alpha)\right] .
$$

Hence for the proof of (4.3.18) it suffices to find for $d=45, \alpha \in\{\pi / 4,(7 / 20) \pi\}$ a $\zeta_{*}(\alpha)$ such that (4.3.26) is satisfied for $\zeta=\zeta_{*}(\alpha)$ and an $m \in \mathbb{N}$ such that the right hand side in (4.3.28) is $\geq 1 / 2$. It can be easily verified by some numerical computations that $\zeta_{*}(\pi / 4)=1 / 10, m=30$ and $\zeta_{*}((7 / 20) \pi)=1 / 1000, m=5000$ meet the requirements for $\alpha=\pi / 4$ and $\alpha=(7 / 20) \pi$.

Obviously, theorem 4.3.1 is an immediate consequence of lemma 4.3 .1 and lemma 4.3.2.

In order to obtain a result for the volume of $D_{1}^{2}$ similar to that one stated in corollary 4.3.1 for $V\left(D_{1}^{1}\right)$ we can proceed completely analogously as in the case $D_{1}^{1}$. First observe that

$$
V\left(D_{1}^{2}\right)=\sum_{i=1}^{2} \int_{0}^{1} V\left(\left(r y^{i}+N\left(U, \operatorname{conv}\left\{0,2 y^{i}\right\}\right)\right) \cap D_{1}\right) d r,
$$

where $N\left(U, \operatorname{conv}\left\{0,2 y^{i}\right\}\right)$ denotes the normal cone of the edge $\operatorname{conv}\left\{0,2 y^{i}\right\}$ with respect to $U=\operatorname{conv}\left\{0,2 y^{1}, 2 y^{2}\right\}$. For $i=1,2$ and $z \in N\left(U, \operatorname{conv}\left\{0,2 y^{i}\right\}\right) \cap S^{d-1}$ we define $h_{i}(r, z)=\max \left\{h \in \mathbb{R}^{\geq 0}: r y^{i}+h z \in D_{1}\right\}$ and $r_{i, z}=\max \left\{r \in \mathbb{R}^{\geq 0}\right.$ : $\left.h_{i}(r, z) \geq 1, r \leq 1\right\}$. Using polar coordinates we get (cf. (4.3.1)):

$$
V\left(D_{1}^{2}\right) \geq \frac{1}{d-1} \sum_{i=1}^{2} \int_{S^{d-1} \cap N\left(U, \operatorname{conv}\left\{0,2 y^{i}\right\}\right)} \int_{0}^{r_{i, z}} h_{i}(r, z)^{d-1} d r d z .
$$

For $z \in N\left(U, \operatorname{conv}\left\{0,2 y^{i}\right\}\right) \cap S^{d-1}$ we have to estimate $\int_{0}^{r_{i, z}} h_{i}(r, z)^{d-1} d r$. To this end we must adjust some of the functions defined in definition 4.3.1:

Definition 4.3.2 For $\phi \in[0, \pi / 2)$ and $0 \leq \zeta \leq \min \{2 \sin (\phi), 2 \cos (\phi)\}$ let

$$
\begin{aligned}
\tilde{g}_{1}(\phi, \zeta, d) & =\int_{0}^{\mu(\phi, \zeta)}\left(r \frac{\zeta}{\sqrt{4-\zeta^{2}}}+\frac{2}{\sqrt{4-\zeta^{2}}}\right)^{d-1} d r \\
\tilde{g}_{2}(\phi, \zeta, d) & =\int_{\mu(\phi, \zeta)}^{\sqrt{(2-\zeta) /(2+\zeta)}}\left(r \frac{\sin (\phi)-1}{\sin (\phi)} \sqrt{\frac{2+\zeta}{2-\zeta}}+\frac{1}{\sin (\phi)}\right)^{d-1} d r \\
\tilde{g}_{3}(\phi, \zeta, d) & =g_{1}(\phi, \zeta, d)+g_{2}(\phi, \zeta, d), \\
\tilde{g}(\phi, d) & =\min \left\{g_{3}(\phi, \zeta, d): 0 \leq \zeta \leq \min \{2 \sin (\phi), 2 \cos (\phi)\}\right\},
\end{aligned}
$$




$$
\tilde{p}(\phi, d)=\int_{0}^{\frac{1-\sin (\phi)}{\cos (\phi)}}\left(-r \frac{\cos (\phi)}{\sin (\phi)}+\frac{1}{\sin (\phi)}\right)^{d-1} d r .
$$

With these notation we have:

Lemma 4.3.3 Let $\phi \in[0, \pi / 2)$ and $z \in N\left(U, \operatorname{conv}\left\{0,2 y^{i}\right\}\right) \cap S^{d-1}$. For $i=1,2$ one has

$$
\int_{0}^{r_{i, z}} h_{i}(r, z)^{d-1} \geq\left\{\begin{array}{lr}
\tilde{g}(\phi, d) & : \phi<\pi / 4 \\
\min \{\tilde{g}(\phi, d), \tilde{p}(\phi, d)\}: \pi / 4 \leq \phi
\end{array}\right.
$$

This lemma can be proved in the same way as lemma 4.3.1. Indeed, we just have to set $|w|=1$ and to replace the angle $\alpha$ by $\phi$ in (4.3.7). Therefore, we omit a detailed proof. Analogously to the proof of lemma 4.3 .2 we can estimate the function $\tilde{g}(\phi, d)$ and get:

Lemma 4.3.4 Let $d \geq 45$. Then

$$
\tilde{g}(\phi, d) \begin{cases}=1 & : \phi \leq \pi / 4 \\ \geq \min \{1, \tilde{p}(\phi, d)\} & : \pi / 4 \leq \phi \leq(7 / 20) \pi .\end{cases}
$$

Clearly, the last two lemmas imply (cf. theorem 4.3.1):

Theorem 4.3.2 Let $d \geq 45$ and $z \in N\left(U, \operatorname{conv}\left\{0,2 y^{i}\right\}\right) \cap S^{d-1}$. For $i=1,2$ one has

$$
\int_{0}^{r_{i, z}} h_{i}(r, z)^{d-1} d r \geq \tilde{f}_{2}(\phi, d)= \begin{cases}1 & : \phi<\pi / 4, \\ \min \{1, \tilde{p}(\phi, d)\}: \pi / 4 \leq \phi \leq(7 / 20) \pi .\end{cases}
$$

Finally, on account of (4.3.29) we obtain:

Corollary 4.3.2 Let $d \geq 45$ and $\phi \in[0,(7 / 20) \pi]$. Then

$$
V\left(D_{1}^{2}\right) \geq \kappa_{d-1} \cdot \tilde{f}_{2}(\phi, d)
$$

\subsection{Proof of theorem 45}

Before we start with the proof we remark that the functions $f_{2}(\alpha, d), \tilde{f}_{2}(\phi, d)$ (cf. theorem 4.3.1, theorem 4.3.2) are monotonely decreasing in $\alpha, \phi$, respectively and monotonely increasing in $d$. Hence for $\alpha, \phi \leq \alpha^{*}=(41 / 120) \pi$ and $d \geq 45$ we have $f_{2}(\alpha, d) \geq f_{2}\left(\alpha^{*}, 45\right)=1 / 2$ and $\tilde{f}_{2}(\phi, d) \geq \tilde{f}_{2}\left(\alpha^{*}, 45\right)=1$. Thus 


$$
\begin{aligned}
& f_{2}(\alpha, d)=\frac{1}{2}, \\
& \tilde{f}_{2}(\phi, d)=1,
\end{aligned} \quad \text { for } \alpha, \phi \leq \alpha^{*}, d \geq 45 .
$$

We recall that the quotient $\kappa_{d-1} / \kappa_{d}$ is strictly monotonely increasing in $d$.

Proof of theorem 45. As in the proof of theorem 3.1.1 we distinguish three cases depending on the angle $\phi$ and the sign of $\left\langle y^{1}, y^{2}\right\rangle$.

i) $\phi<\pi / 3$ and $\left\langle y^{1}, y^{2}\right\rangle \geq 0$.

So we have the "end of the sausage" case and by lemma 3.1.2, lemma 3.1.5, corollary 4.3.1 and corollary 4.3.2 we get

$$
\begin{aligned}
V\left(D_{1}\right) & \geq V\left(D_{1}^{1}\right)+V\left(D_{1}^{2}\right)+V\left(D_{1}^{3}\right) \\
& \geq \phi f_{2}(\alpha, d) \kappa_{d-2}+\tilde{f}_{2}(\phi, d) \kappa_{d-1}+\frac{1-\phi / \pi}{2} \kappa_{d} .
\end{aligned}
$$

Since $\alpha \leq \phi<\pi / 3$ we obtain by (4.4.1):

$$
\begin{aligned}
V\left(D_{1}\right) & \geq \kappa_{d-1}+\frac{1}{2} \kappa_{d}+\frac{\phi}{2} \kappa_{d}\left(\frac{\kappa_{d-2}}{\kappa_{d}}-\frac{1}{\pi}\right) \\
& \geq \kappa_{d-1}+\frac{1}{2} \kappa_{d}+\frac{\phi}{2} \kappa_{d}\left(\frac{\kappa_{43}}{\kappa_{45}}-\frac{1}{\pi}\right) \geq \kappa_{d-1}+\frac{1}{2} \kappa_{d}, \quad d \geq 45 .
\end{aligned}
$$

ii) $\phi<\pi / 3$ and $\left\langle y^{1}, y^{2}\right\rangle<0$.

First assume $\phi \leq \pi / 4$. Then by lemma 3.1.2, lemma 3.1.6, corollary 4.3.1, corollary 4.3 .2 and (4.4.1):

$$
\begin{aligned}
V\left(D_{1}\right) & \geq V\left(D_{1}^{1}\right)+V\left(D_{1}^{2}\right)+V\left(D_{1}^{4}\right) \\
& \geq \phi f_{2}(\alpha, d) \kappa_{d-2}+\tilde{f}_{2}(\phi, d) \kappa_{d-1}+\frac{\cos (\phi)-\sin (\phi)}{\cos (\phi / 2)} \kappa_{d-1} \\
& \geq 2 \kappa_{d-1}+\kappa_{d-1}\left(\frac{\phi}{2} \frac{\kappa_{d-2}}{\kappa_{d-1}}+\frac{\cos (\phi)-\sin (\phi)}{\cos (\phi / 2)}-1\right) .
\end{aligned}
$$

Calculating the second derivative shows that the function in the brackets is concave with respect to $\phi, \phi \leq \pi / 2$. Hence for $d \geq 45$ and $\phi \leq \pi / 4$ :

$$
V\left(D_{1}\right) \geq \min \left\{2 \kappa_{d-1}, 2 \kappa_{d-1}+\kappa_{d-1}\left(\frac{\pi}{8} \frac{\kappa_{43}}{\kappa_{44}}-1\right)\right\} \geq 2 \kappa_{d-1} .
$$

Now let $\pi / 4 \leq \phi<\pi / 3$. As above we obtain for $d \geq 45$ :

$$
\begin{aligned}
V\left(D_{1}\right) & \geq V\left(D_{1}^{1}\right)+V\left(D_{1}^{2}\right) \geq \phi f_{2}(\alpha, d) \kappa_{d-2}+\tilde{f}_{2}(\phi, d) \kappa_{d-1} \\
& \geq 2 \kappa_{d-1}+\kappa_{d-1}\left(\frac{\pi}{8} \frac{\kappa_{d-2}}{\kappa_{d-1}}-1\right) \geq 2 \kappa_{d-1}+\kappa_{d-1}\left(\frac{\pi}{8} \frac{\kappa_{43}}{\kappa_{44}}-1\right) \\
& >2 \kappa_{d-1} .
\end{aligned}
$$

Together with (4.4.2) it follows $V\left(D_{1}\right) \geq 2 \kappa_{d-1}$ for $d \geq 45$. 
iii) $\phi \geq \pi / 3$.

Here we distinguish two cases depending on the angle $\alpha$. In both cases we have to evaluate certain integrals which involves the function $q(d, l, m, r)$ from definition 4.1.1. Instead of determining the exact value of $q(d, l, m, r)$ we use the following upper bound:

$$
q(d, l, m, r) \leq \frac{M(d, l, k(m, r), m, r)}{K(d, l, k(m, r), m, r)},
$$

where $k(m, r)$ is the smallest integer greater $\left(1+r^{2}\right) /\left(1-r^{2}\right)+m$. If $k(m, r) \notin$ $Q(d, l, m, r)$ then we use the trivial upper bound $\infty$. The numerical calculations of the integrals were carried out by the program Mathematica ${ }^{3}$ with a working precision of 40 digits.

Let $\alpha \leq \alpha^{*}=(41 / 120) \pi$. For $V\left(D_{1}^{2}\right)$ we use corollary 4.1 .2 and for $V\left(D_{1}^{1}\right)$ we use corollary 4.3.1. On account of $\phi \geq \pi / 3$ and (4.4.1) we get for $d \geq 45$ :

$$
V\left(D_{1}\right) \geq V\left(D_{1}^{1}\right)+V\left(D_{1}^{2}\right) \geq \frac{\pi}{6} \kappa_{d-2}+\kappa_{d-1} \int_{0}^{1} \frac{1}{1+q(d, 2,1, r)} d r .
$$

Now $\int_{0}^{1} 1 /(1+q(45,2,1, r)) d r \geq 0.65837342$ and as $q(d, 2,1, r)$ is monotonely decreasing in $d$ (cf. remark 4.1.1) we have

$$
\begin{aligned}
V\left(D_{1}\right) & \geq 2 \kappa_{d-1}+\kappa_{d-1}\left(\frac{\pi}{6} \frac{\kappa_{d-2}}{\kappa_{d-1}}-1.34162658\right) \\
& \geq 2 \kappa_{d-1}+\kappa_{d-1}\left(\frac{\pi}{6} \frac{\kappa_{43}}{\kappa_{44}}-1.34162658\right)>2 \kappa_{d-1}, \quad d \geq 45
\end{aligned}
$$

For $\alpha \geq \alpha^{*}$ we apply theorem 4.2.1. First we check that for $d \geq 45$ the restriction (4.2.5) is satisfied: Since the functions $w_{i}(d), 1 \leq i \leq 3$, are monotonely increasing in $d$ we have $w_{1}(d) / w_{3}(d) \leq 1 / w_{3}(45)$ for $d \geq 45$. For the values of $w_{i}(45)$ we find

$$
w_{1}(45) \geq 0.63554953, w_{2}(45) \geq 0.21627735 \text { and } w_{3}(45) \geq 0.089235868 \text {. }
$$

Hence for $d \geq 45$ we have $w_{1}(d) / w_{3}(d)<12<2 \pi \kappa_{d-3} / \kappa_{d-1}$ and (4.2.5) is satisfied. Theorem 4.2.1 yields

$$
V\left(D_{1}\right) \geq f_{1}\left(\alpha^{*}, \gamma^{*} / 2, \gamma^{*}, d\right)
$$

where $\gamma^{*} \in[0, \pi / 2]$ is given by $\cos \left(\gamma^{*}\right) / \cos \left(\gamma^{*} / 2\right)=\cos \left(\alpha^{*}\right)$, i.e. $\gamma^{*} \sim 1.1274629$. By (4.2.4) we see that $f_{1}\left(\alpha^{*}, \gamma^{*} / 2, \gamma^{*}, d\right) / \kappa_{d-1}$ is monotonely increasing in $d$ and

\footnotetext{
${ }^{3}$ (c) 1988,1991,1992 von Wolfram Research Inc.
} 
with $f_{1}\left(\alpha^{*}, \gamma^{*} / 2, \gamma^{*}, 45\right) / \kappa_{44} \geq 2.00510026$ we get

$$
\begin{aligned}
V\left(D_{1}\right) & \geq 2 \kappa_{d-1}+\kappa_{d-1}\left(\frac{f_{1}\left(\alpha^{*}, \gamma^{*} / 2, \gamma^{*}, d\right)}{\kappa_{d-1}}-2\right) \\
& \geq 2 \kappa_{d-1}+\kappa_{d-1}\left(\frac{f_{1}\left(\alpha^{*}, \gamma^{*} / 2, \gamma^{*}, 45\right)}{\kappa_{44}}-2\right) \\
& >2 \kappa_{d-1}, \quad d \geq 45 .
\end{aligned}
$$

Together with (4.4.3) we obtain also in this case $V\left(D_{1}\right) \geq 2 \kappa_{d-1}$ for $d \geq 45$.

Now the first case $\left(\phi<\pi / 3,\left\langle y^{1}, y^{2}\right\rangle \geq 0\right)$ can occur at most twice and this shows that a sausage configuration is a best possible configuration for $d \geq 45$ (cf. proof of theorem 3.1.1). Furthermore, it follows immediately from the proof that a sausage configuration is the only best possible configuration for $d \geq 45$.

Remark. The crucial point for the dimension 45 is the case $\phi \geq \pi / 3$ of the above proof. There we have to find an angle $\alpha^{*}$ and a dimension $d$ such that

- for $\alpha \leq \alpha^{*}$ the volume estimate of $V\left(D_{1}\right)$ based on corollary 4.3 .1 ("perturbing points") and on corollary 4.1.2 is sufficient, and

- for $\alpha \geq \alpha^{*}$ the estimate of $V\left(D_{1}\right)$ given by theorem 4.2 .1 ("3-dimensional sections") is large enough.

With computer aided methods one can "show" that the cases i) and ii) of the proof are harmless for dimension $\geq 30$. 


\section{Lattice packings}

In this chapter we consider the relation between finite lattice packings and infinite lattice packings in the sense of chapter 2. The basic definitions will be given in section 5.1; in particular we will introduce a critical lattice parameter for convex bodies by analogy with definition 2.1.2. An upper bound for the critical parameter will be established in section 5.3. This estimate is based on a result from section 5.2 , where we study a certain problem in the Geometry of Numbers, that may be of interest in its own.

\section{$5.1 \quad$ Introduction}

In section 1.1 we have already given an outline of the infinite lattice packing problem and the following notation has been introduced: For $K \in \mathcal{K}^{d}, \mathcal{P}^{*}(K)$ denotes the set of all packing lattices with respect to $K$, and the density of a densest lattice packing of $K$ is denoted by $\delta^{*}(K)$.

Now, what is a finite lattice packing? In order to obtain a relation between finite and infinite lattice packings it seems natural to regard

$$
\mathcal{P}^{*}(K, n)=\left\{C \in \mathcal{P}(K, n): \text { there exists a } \Lambda \in \mathcal{P}^{*}(K) \text { with } C \subset \Lambda\right\}
$$

as the set of all finite lattice packing sets or lattice packing configurations of cardinality $n$. So $C+K$, for $C \in \mathcal{P}^{*}(K, n)$, is called a finite lattice packing of $K$ (of cardinality $n$ ). For example, the first packing set $\left\{0, x^{1}, x^{2}\right\}$ in figure 5.1.1 is a lattice packing set of cardinality 3 for the 2-dimensional unit disk. The set is part of the hexagonal lattice, whereas the second set $\left\{0, x^{1}, x^{2}\right\}$ is not a finite lattice packing set since any lattice containing $x^{1}, x^{2}$ must also contain the point $z=x^{1}+x^{2}$.

As in the non-lattice case we are interested in densest packings. By analogy with definition 2.1.1 we define, for a parameter $\rho>0$, the following parameterized densities. 

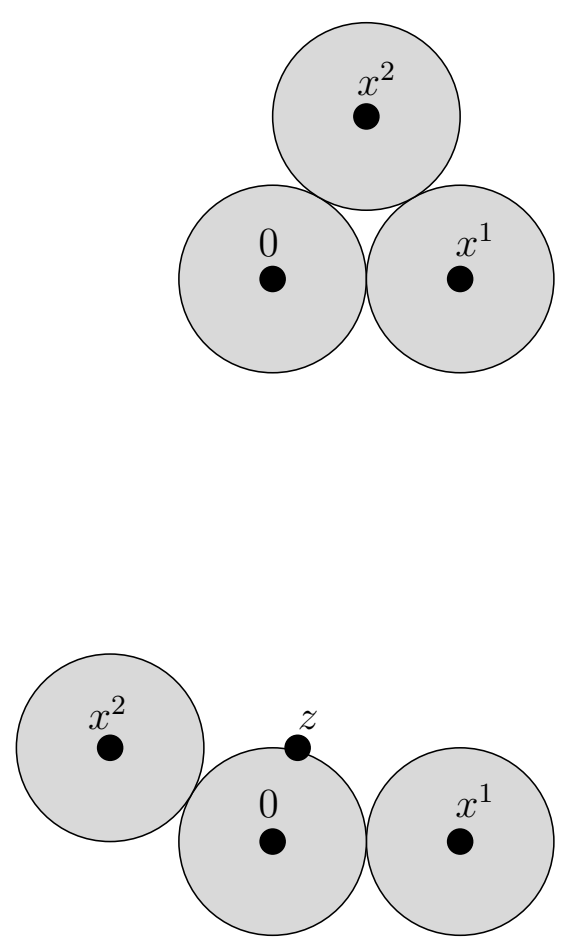

Figure 5.1.1.

Definition 5.1.1 Let $n \in \mathbb{N}$ and $K \in \mathcal{K}^{d}$. For $\rho \in \mathbb{R}^{>0}$ let

$$
\begin{aligned}
\delta_{\rho}^{*}(K, n) & =\sup \left\{\delta_{\rho}(K, C): C \in \mathcal{P}^{*}(K, n)\right\} \\
\delta_{\rho}^{*}(K) & =\limsup _{n \rightarrow \infty} \delta_{\rho}^{*}(K, n) .
\end{aligned}
$$

$\delta_{\rho}^{*}(K, n)$ is the density of a densest finite lattice packing of cardinality $n$ with respect to the "density measure" induced by $\delta_{\rho}(K, C)$. $\delta_{\rho}^{*}(K)$ may be considered as the "density" of a densest finite lattice packing as $n$ tends to infinity.

It is not hard to see that we may replace "sup" in the definition of $\delta_{\rho}^{*}(K, n)$ by "max". In order to give a little insight in the special nature of lattice packings we give a proof.

Proposition 5.1.1 Let $n \in \mathbb{N}, K \in \mathcal{K}^{d}$ and $\rho>0$. Then

$$
\delta_{\rho}^{*}(K, n)=\max \left\{\delta_{\rho}(K, C): C \in \mathcal{P}^{*}(K, n)\right\}
$$

Proof. Let $C^{m}=\left\{x^{1_{m}}, \ldots, x^{n_{m}}\right\} \in \mathcal{P}^{*}(K, n)$ with $\lim _{m \rightarrow \infty} n V(K) / V\left(\operatorname{conv}\left(C^{m}\right)+\right.$ $\rho K)=\delta_{\rho}^{*}(K, n)$. Without loss of generality we may assume $0 \in C^{m}$. From the definition of $\delta_{\rho}^{*}(K, n)$ it follows that the diameter of $\operatorname{conv}\left(C^{m}\right)$ is bounded from above by a constant only depending on $n, d$ and $K$. Hence every sequence 
$\left\{x^{j_{m}}\right\}_{m \in \mathbb{N}}, 1 \leq j \leq n$, is bounded and thus, by the theorem of BolzANoWeIERSTRAss, there exists a subsequence $\left\{m_{k}\right\}_{k \in \mathbb{N}}$ and points $x^{1}, \ldots, x^{n} \in E^{d}$ with $x^{j_{m_{k}}} \rightarrow x^{j}$ as $k \rightarrow \infty$.

Let $C=\left\{x^{1}, \ldots, x^{n}\right\}$. With respect to the HAUssDorf metric we have $\operatorname{conv}\left(C^{m_{k}}\right) \rightarrow \operatorname{conv}(C)$ for $k \rightarrow \infty$ and by the continuity of the mixed volumes (cf. [Sch93]) we obtain $\delta_{\rho}^{*}(K)=n V(K) / V(\operatorname{conv}(C)+\rho K)$. It remains to show that $C \in \mathcal{P}^{*}(K, n)$. To this end let $\Lambda\left(C^{m_{k}}\right)=\left\{x \in E^{d}: x=\right.$ $\left.\sum_{i=1}^{n} z_{i} x^{i_{m_{k}}}, z_{i} \in \mathbb{Z}, 1 \leq i \leq n\right\}$. Since each $C^{m_{k}}$ is part of a packing lattice of $K$ we have $\Lambda\left(C^{m_{k}}\right) \in \mathcal{P}(K)$. Now, $\lim _{k \rightarrow \infty} \sum_{i=1}^{n} z_{i} x^{i_{m_{k}}}=\sum_{i=1}^{n} z_{i} x^{i}$ and thus $\Lambda(C)=\left\{x \in E^{d}: x=\sum_{i=1}^{n} z_{i} x^{i}, z_{i} \in \mathbb{Z}, 1 \leq i \leq n\right\}$ is a packing set for $K$. Hence $\Lambda(C)$ is a discrete subgroup of $E^{d}$. In general $\Lambda(C)$ need not be full-dimensional, i.e. we may have $\operatorname{dim}(\Lambda(C))<d$. In this case it is easy to see that $\Lambda(C)$ can be supplemented to a packing lattice $\Lambda$ of $K$.

The influence of the parameter $\rho$ on the shape of a best possible finite lattice packing is the same as in the general case (see section 2.1). Since every sausage configuration in the sense of definition 2.1.3 is also a finite lattice packing set, we may make the following definitions (compare to definitions 2.1.2, 2.1.4, 2.1.5):

Definition 5.1.2 For $K \in \mathcal{K}^{d}$ let

$$
\begin{aligned}
& \rho_{c}^{*}(K)=\inf \left\{\rho \in \mathbb{R}^{>0}: \delta_{\rho}^{*}(K)=\delta^{*}(K)\right\} \\
& \rho_{s}^{*}(K)=\sup \left\{\rho \in \mathbb{R}^{>0}: \delta_{\rho}^{*}(K)=\delta_{\rho}^{s}(K)\right\} .
\end{aligned}
$$

The number $\rho_{c}^{*}(K)$ is called the critical lattice parameter of $K$ and $\rho_{s}^{*}(K)$ is called the sausage lattice parameter of $K$.

It can easily be checked that the statements of theorem 2.1.1, theorem 2.1.2 and theorem 2.1.3 also hold for the functionals $\delta_{\rho}^{*}(K, n), \delta_{\rho}^{*}(K), \rho_{c}^{*}(K)$ and $\rho_{s}^{*}(K)$. So without a needing of a new proof we summarize these properties in the next theorem:

Theorem 5.1.1 Let $n \in \mathbb{N}, K \in \mathcal{K}^{d}$, and let $0<\rho_{s}^{*}(K), \rho_{c}^{*}(K)<\infty$.

i) $\delta_{\rho}^{*}(K, n), \delta_{\rho}^{*}(K)$ are monotonely decreasing in $\rho$.

ii) Let $A: E^{d} \rightarrow E^{d}$ be a non-singular affine transformation. Then $\delta_{\rho}^{*}(A K, n)=\delta_{\rho}^{*}(K, n)$ and $\delta_{\rho}^{*}(A K)=\delta_{\rho}^{*}(K)$.

iii) $\min \left\{\frac{\rho^{1-d}}{d}, \rho^{-d}\right\}<\frac{n}{d(n-1) \rho^{d-1}+\rho^{d}} \leq \delta_{\rho}\left(K, S_{n}(K)\right)$

$$
\leq \delta_{\rho}^{*}(K, n) \leq \max \left\{\rho^{-d}, 1\right\} .
$$

iv) $\delta_{\rho}^{*}(K, n), \delta_{\rho}^{*}(K)$ are continuous in $\rho$. 
v) There exist packing sets $C_{n} \in \mathcal{P}^{*}(K, n), n \in \mathbb{N}$, such that $\limsup _{n \rightarrow \infty} \delta_{\rho}\left(K, C_{n}\right)$ $=\delta^{*}(K)$ for all $\rho \in \mathbb{R}^{>0}$.

vi) $\delta_{\rho}^{*}(K) \geq \delta^{*}(K)$ for all $\rho \in \mathbb{R}^{>0}$.

vii) $\delta_{\rho}^{*}(K)=\delta_{\rho}^{s}(K)$ for $\rho \in\left(0, \rho_{s}^{*}(K)\right]$.

viii) $\delta_{\rho}^{*}(K)=\delta^{*}(K)$ for $\rho \in\left[\rho_{c}^{*}(K), \infty\right)$.

ix) $\rho_{s}^{*}(K) \leq \rho_{c}^{*}(K)$.

x) Let $n \in \mathbb{N}$ and $\rho^{\prime}>0$ with $\delta_{\rho^{\prime}}^{*}(K, n)=\delta_{\rho^{\prime}}\left(K, S_{n}(K)\right)$. Then for $0<$ $\rho<\rho^{\prime}$ each $C \in \mathcal{P}^{*}(K, n)$ with $\delta_{\rho}(K, C)=\delta_{\rho}^{*}(K, n)$ is a densest sausage configuration.

xi) $\delta_{\rho_{c}^{*}(K)}^{s}(K) \leq \delta^{*}(K) \leq \delta_{\rho_{s}^{*}(K)}^{s}(K)$.

From the last inequality we may deduce (cf. corollary 2.1.1):

$$
\left(\rho_{c}^{*}(K)\right)^{1-d} \frac{1}{d} \leq \delta^{*}(K) \leq\left(\rho_{s}^{*}(K)\right)^{1-d} .
$$

Also, as in the non-lattice case we see that any lower bound for $\rho_{s}^{*}(K)$ gives an upper bound for $\delta^{*}(K)$, and any upper bound for $\rho_{c}^{*}(K)$ yields a lower bound for $\delta^{*}(K)$.

Obviously, $\mathcal{P}^{*}(K, n) \subset \mathcal{P}(K, n)$, and thus

$$
\rho_{s}^{*}(K) \geq \rho_{s}(K) .
$$

Hence any lower bound on $\rho_{s}(K)$ is a lower bound of $\rho_{s}^{*}(K)$. Summarizing the results for the sausage parameter of section 2.4 and section 3.1 we have:

Theorem 5.1.2 Let $K \in \mathcal{K}^{d}$.

$$
\begin{aligned}
& \rho_{s}^{*}(K) \geq \frac{1}{32} d^{-1} \frac{r(K)}{R(K)} \geq\left\{\begin{array}{l}
\frac{1}{32} d^{-3 / 2}: \text { for } K=-K, \\
\frac{1}{32} d^{-2} \quad \text { : for general } K .
\end{array},\right. \\
& \liminf _{d \rightarrow \infty} \rho_{s}^{*}\left(B^{d}\right) \geq \sqrt{2} .
\end{aligned}
$$

In general a relation of the form (5.1.2) does not exist for the critical lattice parameter. Indeed, the determination of a "good" upper bound on $\rho_{c}^{*}(K)$ appears to be much harder than in the non-lattice case. Namely, suppose we could prove for centrally symmetric convex bodies the upper bound 2 as in the non-lattice case (cf. corollary 2.3.1), then by (5.1.1) we would have proved a lower bound 
on $\delta^{*}(K)$ that is of the same order of magnitude as the famous MinkowskiHLAWKa bound (cf (1.1.9)). So we can not expect to show $\rho_{c}^{*}(K)<\alpha$ for any $\alpha<2$. Unfortunately, even a proof for $\rho_{c}^{*}(K) \leq 2, K \in \mathcal{K}_{0}^{d}$, is currently out of reach. In section 5.3 we will show

$$
\rho_{c}^{*}(K) \leq \begin{cases}\sqrt{21} / 2 & : \text { for } K=B^{d} \\ 3 & : \text { for } K=-K \\ (3 / 2)(d+1) & : \text { for general } K\end{cases}
$$

At least for the $d$-dimensional unit ball the bound is not too bad with respect to the Minkwoski-Hlawka bound. The proof of this result is prepared by section 5.2 , where we study a certain problem in the theory of infinite lattice packings.

However, in dimension 2 we have much more information. Since $\delta^{*}(K)=\delta(K)$ for $K \in \mathcal{K}^{2}$ (cf. (1.1.11)) it is easy to see that theorem 2.2.1 and theorem 2.2.2 are valid for finite lattice packings. As a consequence of corollary 2.2.2 we obtain:

Theorem 5.1.3 For $K \in \mathcal{K}^{2}$ one has

$$
\frac{3}{4} \leq \rho_{s}(K)=\rho_{s}^{*}(K)=\frac{\delta_{1}^{s}(K)}{\delta(K)}=\rho_{c}^{*}(K)=\rho_{c}(K) \leq 1 .
$$

Proof. Obviously,

$$
\delta^{*}(K)=\delta(K)=\limsup _{n \rightarrow \infty} \delta_{\rho_{c}(K)}(K, n) \geq \limsup _{n \rightarrow \infty} \delta_{\rho_{c}(K)}^{*}(K, n)
$$

and so $\delta_{\rho_{c}(K)}^{*}(K) \leq \delta^{*}(K)$. On account of theorem 5.1.1 vi) and i) this implies $\rho_{c}^{*}(K) \leq \rho_{c}(K)$. With corollary 2.2.2 and (5.1.2) we obtain

$$
\rho_{s}^{*}(K) \geq \rho_{s}(K)=\frac{\delta_{1}^{s}(K)}{\delta(K)}=\rho_{c}(K) \geq \rho_{c}^{*}(K) .
$$

From $\rho_{s}^{*}(K) \leq \rho_{c}^{*}(K)$ we now get the assertion.

Finally we remark that BETKE [Bet93] has proved $\rho_{s}^{*}\left(B^{3}\right)=\rho_{c}^{*}\left(B^{3}\right)$. This indicates that a "lattice" version of the strong sausage conjecture (see section 2.1) might also be true.

\subsection{Lattice refinements}

A well-known problem in the Geometry of Numbers is to find a lattice which is simultaneously a good packing and covering lattice of a given centrally symmetric convex body (cf. [GL87], [EGH89]). To be more precise we define: 
Definition 5.2.1 For $K \in \mathcal{K}_{0}^{d}$ and a lattice $\Lambda \subset E^{d}$ let

$$
\mu(K, \Lambda)=\min \left\{\mu \in \mathbb{R}^{>0}: \Lambda+\mu K=E^{d}\right\} .
$$

$\mu(K, \Lambda)$ is called the inhomogeneous minimum (or covering radius) of $\Lambda$ with respect to $K$.

$\mu(K, \Lambda)$ is the smallest factor $\mu$ of dilation with the property that $\Lambda$ is a covering lattice for $\mu K$. In terms of the distance function (cf. [GL87]) we get

$$
\mu(K, \Lambda)=\max _{z \in E^{d}} \min \left\{f_{K}(z-a): a \in \Lambda\right\}
$$

This means that $\mu(K, \Lambda)$ is the smallest number $\mu$ such that, for each point $z \in E^{d}$, one can find a lattice point $a \in \Lambda$ with $f_{K}(z-a) \leq \mu$. Since $\Lambda+\mu(K, \Lambda) K$ is a covering of the space we have

$$
V(\mu(K, \Lambda) K) \geq \operatorname{det}(\Lambda)
$$

Hence for $\Lambda \in \mathcal{P}^{*}(K)$ follows (cf. (1.1.3)):

$$
\delta(K, \Lambda) \geq\left(\frac{1}{\mu(K, \Lambda)}\right)^{d}
$$

Now the simultaneous packing and covering problem is the task:

Simultaneous packing and covering problem. For $K \in \mathcal{K}_{0}^{d}$ find a packing lattice $\Lambda \in \mathcal{P}^{*}(K)$ such that $\mu(K, \Lambda)$ is minimal.

Here we are dealing with the following "special" version of the above problem:

Lattice refinement problem. For $K \in \mathcal{K}_{0}^{d}$ and $\Lambda \in \mathcal{P}^{*}(K)$ find a packing lattice $\Lambda^{\prime}$ of $K$ with $\Lambda \subset \Lambda^{\prime}$ such that $\mu\left(K, \Lambda^{\prime}\right)$ becomes minimal.

So, starting with an arbitrary packing lattice $\Lambda$ of a centrally symmetric convex body we are looking among all packing lattices containing $\Lambda$ for one whose inhomogeneous minimum is minimal. The existence of such a "minimal" lattice follows from the selection theorem of MAHLER (cf. [Gru93]).

In view of the Minkowski-HLAWKA bound (cf. (1.1.9)) and (5.2.2) one can not expect to solve one of these problems with an inhomogeneous minimum less than 2. However, in 1972 ButLER [But72] showed:

Theorem 5.2.1 (Butler) Let $K \in \mathcal{K}_{0}^{d}$. There exists a packing lattice $\Lambda \in$ $\mathcal{P}^{*}(K)$ with

$$
\mu(K, \Lambda)=2+o(1), d \rightarrow \infty
$$


The proof of Butler is based on a mean value argument. Therefore his result can not be applied to the refinement problem.

But Rogers [Rog50] proved in 1950 that the ratio of the density of a thinnest lattice covering to the density of a densest lattice packing of a centrally symmetric convex body $K \in \mathcal{K}_{0}^{d}$ is at most $3^{d-1}$ (see also [Ban90]). As an immediate consequence of ROGERS' proof of that result we obtain:

Theorem 5.2.2 (Rogers) Let $K \in \mathcal{K}_{0}^{d}$ and $\Lambda \in \mathcal{P}^{*}(K)$. There exists a packing lattice $\Lambda^{\prime} \in \mathcal{P}^{*}(K)$ with $\Lambda \subset \Lambda^{\prime}$ and

$$
\mu\left(K, \Lambda^{\prime}\right)<3
$$

Proof. Let $K \in \mathcal{K}_{0}^{d}$ and $\Lambda \in \mathcal{P}^{*}(K)$ with $\mu(K, \Lambda) \geq 3$. In the following we show the existence of a packing lattice $\Lambda^{\prime} \in \mathcal{P}^{*}(K)$ with $\Lambda \subset \Lambda^{\prime}$ and $\operatorname{det}\left(\Lambda^{\prime}\right)=\frac{1}{3} \operatorname{det}(\Lambda)$. Since the determinant of a packing lattice of $K$ is bounded from below by $V(K)$ (cf. (1.1.3)) this implies the assertion.

By the definition of the inhomogeneous minimum there exists a point $z \in E^{d}$ with

$$
f_{K}(z-g) \geq \mu(K, \Lambda), \quad g \in \Lambda .
$$

Moreover, we have $\mu\left(K, \frac{1}{3} \Lambda\right)=\frac{1}{3} \mu(K, \Lambda)$, and thus there exists a lattice point $a \in \Lambda$ with

$$
f_{K}\left(z-\frac{1}{3} a\right) \leq \frac{1}{3} \mu(K, \Lambda)
$$

Clearly, $\frac{1}{3} a$ is not a point of $\Lambda$ and hence the determinant of the lattice $\Lambda^{\prime}=$ $\Lambda \cup\left(\frac{1}{3} a+\Lambda\right) \cup\left(\frac{2}{3} a+\Lambda\right)$ is one-third of the determinant of $\Lambda$. It remains to show that $\Lambda^{\prime}$ is a packing lattice of $K$, i.e. $f_{K}(u-v) \geq 2, u, v \in \Lambda^{\prime}$. To this end it is sufficient to prove $f_{K}\left(\frac{1}{3} a-g\right) \geq 2$ for all $g \in \Lambda$. Since $f_{K}: E^{d} \rightarrow \mathbb{R}^{\geq 0}$ is a norm we obtain by (5.2.3) and (5.2.4) for $g \in \Lambda$ :

$$
f_{K}\left(\frac{1}{3} a-g\right) \geq f_{K}(z-g)-f_{K}\left(\frac{1}{3} a-z\right) \geq \frac{2}{3} \mu(K, \Lambda) \geq 2 .
$$

Figure 5.2.1 gives an illustration of the proof, where $\Lambda$ is the orthogonal lattice spanned by $x^{1}$ and $x^{2}$ (the shaded disks). The new lattice $\Lambda^{\prime}$ is generated by the points $x^{1}$ and $\frac{1}{3} a$ (the shaded and the blank disks).

Now, based on Rogers idea, we get a slightly better bound for the special case $K=B^{d}$.

Theorem 5.2.3 Let $\Lambda \in \mathcal{P}^{*}\left(B^{d}\right)$. There exists a packing lattice $\Lambda^{\prime} \in \mathcal{P}^{*}\left(B^{d}\right)$ with $\Lambda \subset \Lambda^{\prime}$ and

$$
\mu\left(B^{d}, \Lambda^{\prime}\right)<\sqrt{21} / 2 \sim 2.2913
$$




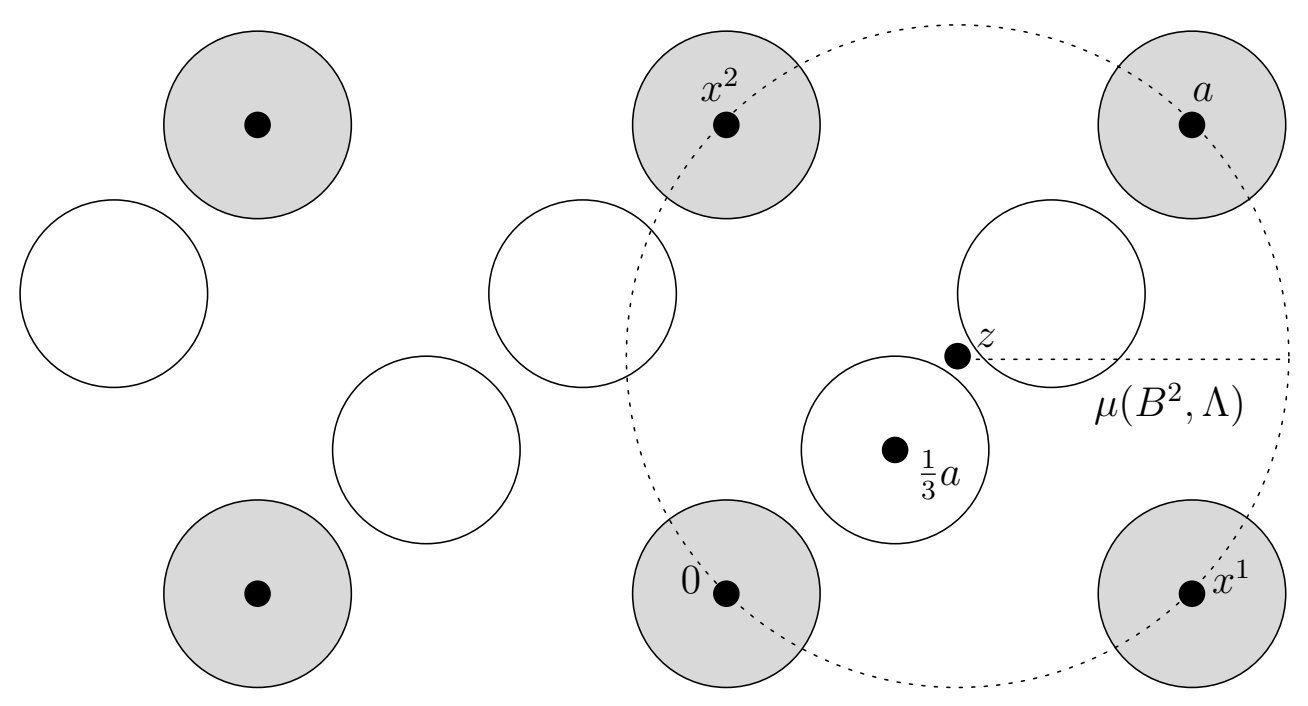

Figure 5.2.1.

Proof. Let $\Lambda \in \mathcal{P}^{*}\left(B^{d}\right)$ with $\mu=\mu\left(B^{d}, \Lambda\right) \geq \sqrt{21} / 2$. We construct a lattice $\Lambda^{\prime}$ containing $\Lambda$ such that $\operatorname{det}\left(\Lambda^{\prime}\right) \leq \frac{1}{2} \operatorname{det}(\Lambda)$. To do this we consider not only points of the lattice $\frac{1}{3} \Lambda$ as in the proof of theorem 5.2.2, but also points of the lattice $\frac{1}{2} \Lambda$. First we note that there exists a $z \in E^{d}$ with (cf. (5.2.3)):

$$
|z-g| \geq \mu, \quad g \in \Lambda \text {. }
$$

Let $\beta=\sqrt{21 / 5}$. We distinguish two cases.

I) There exists an $a \in \Lambda$ with

$$
\left|z-\frac{1}{2} a\right| \leq \frac{\mu}{\beta}
$$

Since $\beta>1$ the point $\frac{1}{2} a$ is not a lattice point of $\Lambda$ and thus the determinant of the lattice $\Lambda^{\prime}=\Lambda \cup\left(\frac{1}{2} a+\Lambda\right)$ is one-half of $\operatorname{det}(\Lambda)$. To show that $\Lambda^{\prime}$ is a packing lattice of $B^{d}$ it suffices to prove $\left|\frac{1}{2} a-g\right| \geq 2$ for all $g \in \Lambda$. By (5.2.5) we get

$$
\begin{aligned}
& \left|\frac{1}{2} a-g+\frac{1}{2} g-\frac{1}{2} z\right|^{2} \geq \frac{\mu^{2}}{4} \\
\Leftrightarrow & \left|\frac{1}{2} a-g\right|^{2}+\frac{1}{4}|g-z|^{2}+\left\langle\frac{1}{2} a-g, g-z\right\rangle \geq \frac{\mu^{2}}{4} .
\end{aligned}
$$

On the other hand we have by (5.2.6):

$$
\begin{aligned}
& \left|\frac{1}{2} a-g+g-z\right|^{2} \leq \frac{\mu^{2}}{\beta^{2}} \\
\Leftrightarrow & \left|\frac{1}{2} a-g\right|^{2}+|g-z|^{2}+2\left\langle\frac{1}{2} a-g, g-z\right\rangle \leq \frac{\mu^{2}}{\beta^{2}} .
\end{aligned}
$$


Substituting $\left\langle\frac{1}{2} a-g, g-z\right\rangle$ from (5.2.8) in (5.2.7) yields on account of $|g-z|^{2} \geq \mu^{2}$ :

$$
\left|\frac{1}{2} a-g\right|^{2} \geq \mu^{2}-\frac{\mu^{2}}{\beta^{2}} \geq 4
$$

II) There exists no $g \in \Lambda$ such that (5.2.6) is satisfied. Then we have

$$
\left|\frac{2}{3} z-\frac{1}{3} g\right|=\frac{2}{3}\left|z-\frac{1}{2} g\right| \geq \frac{2}{3} \frac{\mu}{\beta}, \quad g \in \Lambda .
$$

Now, let $a \in \Lambda$ be a lattice point with (cf. (5.2.4)):

$$
\left|z-\frac{1}{3} a\right| \leq \frac{\mu}{3}
$$

We consider the lattice $\Lambda^{\prime}=\Lambda \cup\left(\frac{1}{3} a+\Lambda\right) \cup\left(\frac{2}{3} a+\Lambda\right)$. Clearly, $\operatorname{det}\left(\Lambda^{\prime}\right)=\frac{1}{3} \operatorname{det}(\Lambda)$ (cf. proof of theorem 5.2.2) and it remains to show $\Lambda^{\prime} \in \mathcal{P}^{*}\left(B^{d}\right)$. To this end we proceed as in the first case. Let $g \in \Lambda$. From (5.2.9) we get

$$
\begin{gathered}
\left|\frac{2}{3} z-\frac{2}{3} g+g-\frac{1}{3} a\right|^{2} \geq \frac{4}{9} \frac{\mu^{2}}{\beta^{2}} \\
\Leftrightarrow \quad\left|g-\frac{1}{3} a\right|^{2}+\frac{4}{9}|z-g|^{2}+\frac{4}{3}\left\langle g-\frac{1}{3} a, z-g\right\rangle \geq \frac{4}{9} \frac{\mu^{2}}{\beta^{2}}
\end{gathered}
$$

and from (5.2.10):

$$
\begin{aligned}
& \left|z-g+g-\frac{1}{3} a\right|^{2} \leq \frac{\mu^{2}}{9} \\
\Leftrightarrow & \left|g-\frac{1}{3} a\right|^{2}+|z-g|^{2}+2\left\langle g-\frac{1}{3} a, z-g\right\rangle \leq \frac{\mu^{2}}{9} .
\end{aligned}
$$

Substituting $\left\langle g-\frac{1}{3} a, z-g\right\rangle$ from (5.2.12) in (5.2.11) yields on account of $|z-g|^{2} \geq$ $\mu^{2}$ :

$$
\left|g-\frac{1}{3} a\right|^{2} \geq \frac{4}{9} \mu^{2}\left(\frac{3}{\beta^{2}}+1\right) \geq 4 .
$$

In any case we obtain a lattice $\Lambda^{\prime} \in \mathcal{P}^{*}\left(B^{d}\right)$ containing $\Lambda$ with $\operatorname{det}\left(\Lambda^{\prime}\right) \leq \frac{1}{2} \operatorname{det}(\Lambda)$. Since the determinant of a packing lattice $\Lambda \in \mathcal{P}^{*}\left(B^{d}\right)$ is bounded from below, this "construction" gives a lattice of the required form after finite many steps.

\section{Remarks.}

- For sphere lattice packings the inhomogeneous minimum is the radius of the so called "deep holes" (cf. [CS93]). The above result says that deep holes in a "good" packing lattice can not be "too deep". 
- In view of (5.2.2), theorem 5.2.3 implies that for every packing lattice $\Lambda \in$ $\mathcal{P}^{*}\left(B^{d}\right)$ there exists a refinement $\Lambda^{\prime} \in \mathcal{P}^{*}\left(B^{d}\right), \Lambda \subset \Lambda^{\prime}$, with

$$
\delta\left(B^{d}, \Lambda^{\prime}\right) \geq\left(\frac{1}{2.3}\right)^{d}
$$

In particular we obtain the lower bound $2.3^{-d}$ for a densest lattice sphere packing. Clearly this bound is worse than the bound given by the theorem of Minkowski-HlawkA, but on the other hand the proof is simple and in a certain way constructive. Indeed, one can show that starting with appropriate orthogonal lattices one can construct densest packing lattices in up to 8 dimensions by successive refinements (cf. [Hen94]).

\subsection{An upper bound for the critical lattice parameter}

Before we can state the bound we have to introduce the so called honeycombs or "Wabenzellen".

Definition 5.3.1 Let $K \in \mathcal{K}_{0}^{d}$ and $\Lambda \subset E^{d}$ be a lattice.

$$
H(K, \Lambda)=\left\{x \in E^{d}: f_{K}(x) \leq f_{K}(x-a), \quad \forall a \in \Lambda\right\}
$$

is called the honeycomb of $K$ with respect to $\Lambda$.

$H\left(B^{d}, \Lambda\right)$ is also called the Dirichlet-Voronö̈-cell of the lattice $\Lambda$. The next lemma collects some elementary properties of honeycombs.

Lemma 5.3.1 Let $K \in \mathcal{K}_{0}^{d}$ and $\Lambda \subset E^{d}$ be a lattice.

i) $H(K, \Lambda)$ is a compact centrally symmetric ray set with $H(K, \Lambda)+\Lambda=E^{d}$.

ii) $\mu(K, \Lambda)=\min \left\{\rho \in \mathbb{R}^{\geq 0}: H(K, \Lambda) \subset \rho K\right\}$.

If, in addition, $K$ is strictly convex then

iii) $\operatorname{int}(H(K, \Lambda))=\left\{x \in E^{d}: f_{K}(x)<f_{K}(x-a), \forall a \in \Lambda \backslash\{0\}\right\}$.

iv) $H(K, \Lambda)$ is a centrally symmetric starbody.

v) $H(K, \Lambda)+\Lambda$ is a tiling and thus $V(H(K, \Lambda))=\operatorname{det}(\Lambda)$. 
We note that a ray set in $E^{d}$ is a non-empty set $S \subset E^{d}$ with the property: if $x \in S$ and $\lambda \in[0,1]$ then $\lambda x \in S$. A star body is a ray set $S$ for which the following conditions hold: $S$ is closed, and for $x \in S, \lambda \in[0,1)$, the point $\lambda x$ is an inner point of $S$. A convex body $K$ is called strictly convex if for all $x, y \in K$ and $\lambda \in(0,1)$ the points $\lambda x+(1-\lambda) y$ are inner points of $K$ (cf. [GL87]).

There exists a proof of some of the properties claimed in the lemma for 2dimensional honeycombs ("domains of action") by BAMBAH, Rogers \& ZASSENHAUS [BRZ64]. Although their arguments also work in higher dimensions for completeness' sake we give a short proof of the lemma.

Proof of Lemma 5.3.1.

i) Since $f_{K}$ is continuous $H(K, \Lambda)$ is closed. By the definition of the inhomogeneous minimum we have

$$
H(K, \Lambda) \subset \mu(K, \Lambda) K .
$$

Thus $H(K, \Lambda)$ is compact. Now let $z \in H(K, \Lambda), \lambda \in[0,1]$, and $g \in \Lambda$ with $f_{K}(\lambda z)>f_{K}(\lambda z-g)$. Then

$$
\begin{aligned}
f_{K}(g-z) & =f_{K}(g-\lambda z+(\lambda-1) z) \leq f_{K}(g-\lambda z)+(1-\lambda) f_{K}(z) \\
& <\lambda f_{K}(z)+(1-\lambda) f_{K}(z)=f_{K}(z) .
\end{aligned}
$$

This contradicts the choice of $z$ and hence $H(K, \Lambda)$ is a ray set. Obviously, $H(K, \Lambda)$ is centrally symmetric and moreover we have

$$
H(K, \Lambda)+\Lambda=E^{d} .
$$

ii) Let $H(K, \Lambda) \subset \beta K$. Then $\beta K+\Lambda$ covers the space (cf. (5.3.2)) and so $\beta \geq \mu(K, \Lambda)$. Together with (5.3.1) we get ii).

iii) It is not hard to see that $\left\{x \in E^{d}: f_{K}(x)<f_{K}(x-a), \forall a \in \Lambda \backslash\{0\}\right\}$ belongs to the interior of $H(K, \Lambda)$. This holds for any centrally symmetric convex body, not necessarily strictly convex. Let $z \in \operatorname{int}(H(K, \Lambda))$ and suppose there exists an $a \in \Lambda$ with $f_{K}(z)=f_{K}(z-a)$. Then

$$
0, a \in z+f_{K}(z) K
$$

and both points lie in the boundary of $z+f_{K}(z) K$. Let $\left\{x \in E^{d}: c^{T} x=0\right\}$ be a supporting hyperplane of $z+f_{K}(z) K$ at the point 0 with $c^{T} x \geq 0$ for all $x \in z+f_{K}(z) K$ and let $\alpha \in(0,1)$ such that $z+\alpha a \in H(K, \Lambda)$. Since $K$ is strictly convex and on account of (5.3.3) we have $c^{T} a>0$. Thus $c^{T} x>0$ for all $x \in z+\alpha a+f_{K}(z) K$. Hence $z+\alpha a \notin f_{K}(z) K$ which is equivalent to $f_{K}(z+\alpha a)>f_{K}(z)$. Furthermore, we see by (5.3.3) that $(1-\alpha) a \in z+f_{K}(z) K$ and thus $f_{K}(z+\alpha a-a) \leq f_{K}(z)$. Altogether we get $f_{K}(z+\alpha a)>f_{K}(z+\alpha a-a)$, which contradicts $z+\alpha a \in H(K, \Lambda)$. 
iv) Let $z \in H(K, \Lambda)$ and $\lambda \in(0,1)$. In view of i) and iii) we have to show $f_{K}(\lambda z)<f_{K}(\lambda z-a)$ for all $a \in \Lambda \backslash\{0\}$. Suppose there exists a $g \in \Lambda \backslash\{0\}$ with $f_{K}(\lambda z) \geq f_{K}(\lambda z-g)$. Clearly, we may assume $g \neq \gamma z, \gamma \in \mathbb{R}$. Since $K$ is strictly convex we have (cf. [Min96]) for $x, y \in E^{d}$ :

$$
f_{K}(x+y)=f_{K}(x)+f_{K}(y) \Leftrightarrow \exists \gamma \in \mathbb{R} \text { with } x=\gamma y .
$$

Hence

$$
f_{K}(g-z)=f_{K}(g-\lambda z+(\lambda-1) z)<f(g-\lambda z)+(1-\lambda) f_{K}(z) \leq f_{K}(z),
$$

in contradiction to the definition of $H(K, \Lambda)$.

v) It suffices to prove $(g+\operatorname{int}(H(K, \Lambda))) \cap(h+\operatorname{int}(H(K, \Lambda)))=\emptyset$ for $g, h \in \Lambda$, $g \neq h$. Suppose the contrary and let $z$ be a point of the intersection. By iii) we get $f_{K}(z-g)<f_{K}(z-h)<f_{K}(z-g)$.

Based on theorems 5.2.2, 5.2.3 and lemma 5.3.1 we obtain by analogy with theorem 2.3.1:

Theorem 5.3.1 Let $n \in \mathbb{N}$ and $K \in \mathcal{K}^{d}$. Then

$$
\delta_{\rho}^{*}(K, n) \leq \delta^{*}(K) \text { for } \rho \geq \begin{cases}\sqrt{21} / 2 & : \text { for } K=B^{d}, \\ 3 & : \text { for } K=-K, \\ (3 / 2)(d+1) & : \text { for general } K .\end{cases}
$$

Proof. First we prove the statement for the class of centrally symmetric strictly convex bodies. From this case we may deduce the general case of symmetric bodies by a standard approximation argument.

Let $K \in \mathcal{K}_{0}^{d}$ be strictly convex and let $\rho \in \mathbb{R}$ with $\rho \geq 3$. By proposition 5.1.1 there exists a lattice packing set $C \in \mathcal{P}^{*}(K, n)$ such that $\delta_{\rho}^{*}(K, n)=$ $n V(K) / V(\operatorname{conv}(C)+\rho K)$. Let $\Lambda \in \mathcal{P}^{*}(K)$ be a packing lattice with $C \subset \Lambda$. In view of theorem 5.2.2 we may assume

$$
\mu(K, \Lambda)<3
$$

With lemma 5.3.1 ii) we obtain

$$
H(K, \Lambda) \subset \rho K
$$

By lemma $5.3 .1 \mathrm{v})$ we know that $H(K, \Lambda)$ generates a lattice tiling with respect to $\Lambda$ and thus

$$
\delta_{\rho}^{*}(K, n)=\frac{n V(K)}{V(\operatorname{conv}(C)+\rho K)} \leq \frac{n V(K)}{V(C+H(K, \Lambda))}=\frac{V(K)}{\operatorname{det}(\Lambda)} \leq \delta^{*}(K) .
$$


By theorem 5.2.3 we may replace $\rho=3$ by $\rho=\sqrt{21} / 2$ in the case $K=B^{d}$.

Now, let $K$ be an arbitrary centrally symmetric body, $\rho \geq 3$ and let $C, \Lambda$ as above. Since the class of strictly convex bodies is dense in the space of convex bodies (cf. [BF34]) there exists a $L \in \mathcal{K}_{0}^{d}, L$ strictly convex, such that (cf. (5.3.4)):

$$
\mu(L, \Lambda)\left(1+\frac{d_{H}(L, K)}{r(K)}\right)<3
$$

where $d_{H}(L, K)$ denotes the Haussdorf distance (cf. [BF34]). In particular we have $L \subset K+d_{H}(L, K) B^{d}$ and so by (5.3.6):

$$
H(L, \Lambda) \subset \mu(L, \Lambda) L \subset \rho K
$$

Hence

$$
\delta_{\rho}^{*}(K, n)=\frac{n V(K)}{V(\operatorname{conv}(C)+\rho K)} \leq \frac{n V(K)}{V(C+H(L, \Lambda))}=\frac{V(K)}{\operatorname{det}(\Lambda)} \leq \delta_{\rho}^{*}(K) .
$$

Finally, let $K$ be an arbitrary convex body, $\rho \geq \frac{3}{2}(d+1)$, and let $C, \Lambda$ as above. Since $C$ is also a packing set for the difference body with respect to $\Lambda$ (cf. (1.1.6)) we obtain from $(5.3 .7),(1.1 .7)$ and the relation $\mathrm{D} K \subset \frac{d+1}{2} K(\mathrm{cf} .[\mathrm{BF} 34])$ :

$$
\begin{aligned}
\delta_{\rho}^{*}(K) & =\frac{n V(K)}{V(\operatorname{conv}(C)+\rho K)} \leq \frac{n V(K)}{V\left(\operatorname{conv}(C)+\frac{2 \rho}{d+1} \mathrm{D} K\right)} \\
& \leq \frac{V(K)}{V(\mathrm{D} K)} \delta_{\frac{2 \rho}{d+1}}^{*}(\mathrm{D} K, n) \leq \frac{V(K)}{V(\mathrm{D} K)} \delta^{*}(\mathrm{D} K)=\delta^{*}(K) .
\end{aligned}
$$

As an immediate consequence of this theorem and theorem 5.1.1 vi) we obtain for the critical lattice parameter:

Corollary 5.3.1 Let $K \in \mathcal{K}^{d}$.

$$
\rho_{c}^{*}(K) \leq \begin{cases}\sqrt{21} / 2 & : \text { for } K=B^{d}, \\ 3 & : \text { for } K=-K, \\ (3 / 2)(d+1) & : \text { for general } K .\end{cases}
$$




\section{Appendix}

In this chapter we will give the proofs of some inequalities used in chapter 4 .

\section{A.1 Partial derivatives of $f_{1,2}, f_{1,3}, f_{2,3}, g_{1,2}, g_{1,3}, g_{2,3}$}

Since $\frac{\delta \arccos (x)}{\delta x}=-1 / \sqrt{1-x^{2}}$ it follows:

Remark A.1.1 Let

$$
\begin{aligned}
& f_{1,2}(\alpha, \beta, \gamma)=\gamma \\
& f_{1,3}(\alpha, \beta, \gamma)=\arccos (\cos (\alpha) \cos (\beta)) \\
& f_{2,3}(\alpha, \beta, \gamma)=\arccos (\cos (\alpha) \cos (\gamma-\beta))
\end{aligned}
$$

Then

$$
\begin{aligned}
\frac{\delta f_{1,2}}{\delta \gamma} & =1, \quad \frac{\delta f_{1,2}}{\delta \beta}=\frac{\delta f_{1,2}}{\delta \alpha}=0 \\
\frac{\delta f_{2,3}}{\delta \gamma} & =\frac{\cos (\alpha) \sin (\gamma-\beta)}{\sqrt{1-\cos ^{2}(\alpha) \cos ^{2}(\gamma-\beta)}}, \quad \frac{\delta f_{2,3}}{\delta \beta}=\frac{-\cos (\alpha) \sin (\gamma-\beta)}{\sqrt{1-\cos ^{2}(\alpha) \cos ^{2}(\gamma-\beta)}} \\
\frac{\delta f_{2,3}}{\delta \alpha} & =\frac{\sin (\alpha) \cos (\gamma-\beta)}{\sqrt{1-\cos ^{2}(\alpha) \cos ^{2}(\gamma-\beta)}}, \\
\frac{\delta f_{1,3}}{\delta \gamma} & =0, \quad \frac{\delta f_{1,3}}{\delta \beta}=\frac{\cos (\alpha) \sin (\beta)}{\sqrt{1-\cos ^{2}(\alpha) \cos ^{2}(\beta)}} \\
\frac{\delta f_{1,3}}{\delta \alpha} & =\frac{\sin (\alpha) \cos (\beta)}{\sqrt{1-\cos ^{2}(\alpha) \cos ^{2}(\beta)}}
\end{aligned}
$$

Remark A.1.2 Let

$$
g_{1,2}(\alpha, \beta, \gamma)=\arccos \left(\frac{-\sin ^{2}(\alpha) \cos (\gamma)+\cos ^{2}(\alpha) \sin (\beta) \sin (\gamma-\beta)}{\sqrt{1-\cos ^{2}(\alpha) \cos ^{2}(\beta)} \sqrt{1-\cos ^{2}(\alpha) \cos ^{2}(\gamma-\beta)}}\right) \text {. }
$$


Then

$$
\begin{aligned}
\frac{\delta g_{1,2}}{\delta \gamma} & =\frac{-\sin (\alpha)}{1-\cos ^{2}(\alpha) \cos ^{2}(\gamma-\beta)} \\
\frac{\delta g_{1,2}}{\delta \beta} & =\frac{-\sin (\alpha) \cos ^{2}(\alpha) \sin (\gamma) \sin (\gamma-2 \beta)}{\left(1-\cos ^{2}(\alpha) \cos ^{2}(\beta)\right)\left(1-\cos ^{2}(\alpha) \cos ^{2}(\gamma-\beta)\right)} \\
\frac{\delta g_{1,2}}{\delta \alpha}(\alpha, \gamma / 2, \gamma) & =\frac{\cos (\alpha) \sin (\gamma)}{1-\cos ^{2}(\alpha) \cos ^{2}(\gamma / 2)}
\end{aligned}
$$

Proof. For abbreviation we set

$$
w_{1}=\sqrt{1-\cos ^{2}(\alpha) \cos ^{2}(\beta)} \quad \text { and } \quad w_{2}=\sqrt{1-\cos ^{2}(\alpha) \cos ^{2}(\gamma-\beta)} .
$$

First we prove the following identity

$$
\frac{1}{\sqrt{1-\left(\frac{-\sin ^{2}(\alpha) \cos (\gamma)+\cos ^{2}(\alpha) \sin (\beta) \sin (\gamma-\beta)}{w_{1} w_{2}}\right)^{2}}}=\frac{w_{1} w_{2}}{\sin (\alpha) \sin (\gamma)} .
$$

This equation is equivalent to

$$
\sin ^{2}(\alpha) \sin ^{2}(\gamma)=w_{1}^{2} w_{2}^{2}-\left(-\sin ^{2}(\alpha) \cos (\gamma)+\cos ^{2}(\alpha) \sin (\beta) \sin (\gamma-\beta)\right)^{2}
$$

Now

$$
\begin{aligned}
w_{1}^{2} w_{2}^{2}-\left(-\sin ^{2}(\alpha)\right. & \left.\cos (\gamma)+\cos ^{2}(\alpha) \sin (\beta) \sin (\gamma-\beta)\right)^{2} \\
= & -\cos ^{2}(\alpha) \cos ^{2}(\beta)-\cos ^{2}(\alpha) \cos ^{2}(\gamma-\beta) \\
& +\cos ^{4}(\alpha) \cos ^{2}(\beta) \cos ^{2}(\gamma-\beta) \\
& -\sin ^{4}(\alpha) \cos ^{2}(\gamma)-\cos ^{4}(\alpha) \sin ^{2}(\beta) \sin ^{2}(\gamma-\beta) \\
& +2 \sin ^{2}(\alpha) \cos ^{2}(\alpha) \sin (\beta) \cos (\gamma) \sin (\gamma-\beta) \\
& 1-\cos ^{2}(\alpha) \cos ^{2}(\beta)-\cos ^{2}(\alpha) \cos ^{2}(\gamma-\beta) \\
& +\cos ^{4}(\alpha) \cos ^{2}(\gamma-\beta) \\
& -\sin ^{4}(\alpha) \cos ^{2}(\gamma)-\cos ^{4}(\alpha) \sin ^{2}(\beta) \\
& +2 \sin ^{2}(\alpha) \cos ^{2}(\alpha) \sin (\beta) \cos (\gamma) \sin (\gamma-\beta) \\
& 1-\cos ^{2}(\alpha) \cos ^{2}(\beta)-\cos ^{2}(\alpha) \cos ^{2}(\gamma-\beta) \\
& +\cos ^{2}(\alpha)\left(\cos ^{2}(\alpha) \cos ^{2}(\gamma-\beta)-\cos ^{2}(\alpha) \sin ^{2}(\beta)\right) \\
& -\sin ^{4}(\alpha) \cos ^{2}(\gamma)+2 \sin ^{2}(\alpha) \cos ^{2}(\alpha) \sin (\beta) \cos (\gamma) \sin (\gamma-\beta) \\
=\quad & -\cos ^{2}(\alpha) \cos ^{2}(\beta)-\cos ^{2}(\alpha) \cos ^{2}(\gamma-\beta) \\
& +\cos ^{2}(\alpha) \cos ^{2}(\gamma-\beta)-\cos ^{2}(\alpha) \sin ^{2}(\beta) \\
& -\sin ^{2}(\alpha) \cos ^{2}(\alpha)\left(\cos ^{2}(\gamma-\beta)-\sin ^{2}(\beta)\right) \\
\cos ^{2}+\sin ^{2}=1 \quad &
\end{aligned}
$$




$$
\begin{aligned}
& -\sin ^{4}(\alpha) \cos ^{2}(\gamma)+2 \sin ^{2}(\alpha) \cos ^{2}(\alpha) \sin (\beta) \cos (\gamma) \sin (\gamma-\beta) \\
& \cos ^{2} \stackrel{+\sin ^{2}=1}{=} \quad \sin ^{2}(\alpha)-\sin ^{2}(\alpha) \cos ^{2}(\alpha)\left(\cos ^{2}(\gamma-\beta)-\sin ^{2}(\beta)\right) \\
& -\sin ^{2}(\alpha) \sin ^{2}(\alpha) \cos ^{2}(\gamma) \\
& +2 \sin ^{2}(\alpha) \cos ^{2}(\alpha) \sin (\beta) \cos (\gamma) \sin (\gamma-\beta) \\
& \cos ^{2}+\sin ^{2}=1 \quad \sin ^{2}(\alpha)-\sin ^{2}(\alpha) \cos ^{2}(\alpha)\left(\cos ^{2}(\gamma-\beta)-\sin ^{2}(\beta)\right) \\
& -\sin ^{2}(\alpha) \cos ^{2}(\gamma)+\sin ^{2}(\alpha) \cos ^{2}(\alpha) \cos ^{2}(\gamma) \\
& +2 \sin ^{2}(\alpha) \cos ^{2}(\alpha) \sin (\beta) \cos (\gamma) \sin (\gamma-\beta) \\
& \cos ^{2}+\sin ^{2}=1 \quad \sin ^{2}(\alpha) \sin ^{2}(\gamma)+\sin ^{2}(\alpha) \cos ^{2}(\alpha) \times \\
& \left(\sin ^{2}(\beta)-\underline{\cos ^{2}(\gamma-\beta)}+\cos ^{2}(\gamma)\right. \\
& +2 \sin (\beta) \cos (\gamma) \sin (\gamma-\beta)) \\
& \cos (x \pm y)=\cos (x) \cos (y) \\
& \stackrel{\mp \sin (x) \sin (y)}{=} \sin ^{2}(\alpha) \sin ^{2}(\gamma)+\sin ^{2}(\alpha) \cos ^{2}(\alpha) \times \\
& \left(\sin ^{2}(\beta)-\cos ^{2}(\gamma) \cos ^{2}(\beta)-\sin ^{2}(\gamma) \sin ^{2}(\beta)\right. \\
& -2 \cos (\gamma) \cos (\beta) \sin (\gamma) \sin (\beta) \\
& \left.+\cos ^{2}(\gamma)+2 \sin (\beta) \cos (\gamma) \sin (\gamma-\beta)\right) \\
& \cos ^{2} \stackrel{+\sin ^{2}=1}{=} \sin ^{2}(\alpha) \sin ^{2}(\gamma)+\sin ^{2}(\alpha) \cos ^{2}(\alpha) \times \\
& \left(2 \cos ^{2}(\gamma) \sin ^{2}(\beta)-2 \cos (\gamma) \cos (\beta) \sin (\gamma) \sin (\beta)\right. \\
& +2 \sin (\beta) \cos (\gamma) \sin (\gamma-\beta)) \\
& =\quad \sin ^{2}(\alpha) \sin ^{2}(\gamma)+2 \sin ^{2}(\alpha) \cos ^{2}(\alpha) \cos (\gamma) \sin (\beta) \times \\
& (\cos (\gamma) \sin (\beta)-\cos (\beta) \sin (\gamma)+\sin (\gamma-\beta)) \\
& \sin (x \pm y)=\sin (x) \cos (y) \\
& \stackrel{\cos (x) \sin (y)}{=} \sin ^{2}(\alpha) \sin ^{2}(\gamma) .
\end{aligned}
$$

Next we prove

$$
\begin{aligned}
& \frac{\sin ^{2}(\alpha) \sin (\gamma)}{w_{1} w_{2} w_{2}^{2}}=\frac{\left(\sin ^{2}(\alpha) \sin (\gamma)+\cos ^{2}(\alpha) \sin (\beta) \cos (\gamma-\beta)\right) w_{1} w_{2}}{w_{1}^{2} w_{2}^{2}}- \\
& \frac{\left(-\sin ^{2}(\alpha) \cos (\gamma)+\cos ^{2}(\alpha) \sin (\beta) \sin (\gamma-\beta)\right) \frac{w_{1}}{w_{2}} \cos ^{2}(\alpha) \cos (\gamma-\beta) \sin (\gamma-\beta)}{w_{1}^{2} w_{2}^{2}}
\end{aligned}
$$

This is equivalent to

$$
\begin{aligned}
& \sin ^{2}(\alpha) \sin (\gamma)=\left(\sin ^{2}(\alpha) \sin (\gamma)+\cos ^{2}(\alpha) \sin (\beta) \cos (\gamma-\beta)\right) w_{2}^{2} \\
& \quad-\left(-\sin ^{2}(\alpha) \cos (\gamma)+\cos ^{2}(\alpha) \sin (\beta) \sin (\gamma-\beta)\right) \cos ^{2}(\alpha) \cos (\gamma-\beta) \sin (\gamma-\beta)
\end{aligned}
$$


Now

$$
\begin{aligned}
&\left(\sin ^{2}(\alpha) \sin (\gamma)+\cos ^{2}(\alpha) \sin (\beta) \cos (\gamma-\beta)\right) w_{2}^{2} \\
&-\left(-\sin ^{2}(\alpha) \cos (\gamma)+\cos ^{2}(\alpha) \sin (\beta) \sin (\gamma-\beta)\right) \times \\
& \cos ^{2}(\alpha) \cos (\gamma-\beta) \sin (\gamma-\beta) \\
&=\quad \sin ^{2}(\alpha) \sin (\gamma)+\cos ^{2}(\alpha) \sin (\beta) \cos (\gamma-\beta) \\
&-\sin ^{2}(\alpha) \sin (\gamma) \cos ^{2}(\alpha) \cos ^{2}(\gamma-\beta) \\
&-\cos ^{4}(\alpha) \sin (\beta) \cos ^{3}(\gamma-\beta) \\
&+\sin ^{2}(\alpha) \cos (\gamma) \cos ^{2}(\alpha) \cos (\gamma-\beta) \sin (\gamma-\beta) \\
&-\cos ^{4}(\alpha) \sin (\beta) \cos (\gamma-\beta) \sin ^{2}(\gamma-\beta) \\
& \sin ^{2}(\alpha) \sin (\gamma)+\cos ^{2}(\alpha) \cos (\gamma-\beta) \times \\
&\left(\sin ^{2}(\beta)-\sin (\alpha) \sin (\gamma) \cos (\gamma-\beta)\right. \\
&-\cos ^{2}(\alpha) \sin (\beta) \cos ^{2}(\gamma-\beta) \\
&\left.+\sin ^{2}(\alpha) \cos (\gamma) \sin (\gamma-\beta)-\cos { }^{2}(\alpha) \sin (\beta) \sin ^{2}(\gamma-\beta)\right) \\
& \sin ^{2}(\alpha) \sin (\gamma)+\cos ^{2}(\alpha) \cos (\gamma-\beta) \times \\
&\left(\sin ^{2}(\beta)-\sin (\alpha) \sin (\gamma) \cos (\gamma-\beta)\right. \\
&-\cos ^{2}(\alpha) \sin (\beta) \\
&\left.+\sin ^{2}(\alpha) \cos (\gamma) \sin (\gamma-\beta)\right) \\
& \sin ^{2}(\alpha) \sin (\gamma)+\cos ^{2}(\alpha) \cos (\gamma-\beta) \sin ^{2}(\alpha) \times \\
&\left(\sin ^{2}(\beta)-\sin (\gamma) \cos ^{2}(\gamma-\beta)+\cos (\gamma) \sin (\gamma-\beta)\right) \\
&= \sin ^{2}(\alpha) \sin (\gamma) \\
&
\end{aligned}
$$

On account of (A.1.11) and (A.1.13) we find for the partial derivative of $g_{1,2}$ with respect to $\gamma$

$$
\begin{aligned}
& \frac{\delta g_{1,2}}{\delta \gamma}=\frac{-1}{\sqrt{1-\left(\frac{-\sin ^{2}(\alpha) \cos (\gamma)+\cos ^{2}(\alpha) \sin (\beta) \sin (\gamma-\beta)}{w_{1} w_{2}}\right)^{2}}} \times \\
& \left(\frac{\left(\sin ^{2}(\alpha) \sin (\gamma)+\cos ^{2}(\alpha) \sin (\beta) \cos (\gamma-\beta)\right) w_{1} w_{2}}{w_{1}^{2} w_{2}^{2}}-\right. \\
& \left.\frac{\left(-\sin ^{2}(\alpha) \cos (\gamma)+\cos ^{2}(\alpha) \sin (\beta) \sin (\gamma-\beta)\right) \frac{w_{1}}{w_{2}} \cos ^{2}(\alpha) \cos (\gamma-\beta) \sin (\gamma-\beta)}{w_{1}^{2} w_{2}^{2}}\right) \\
& =\frac{-w_{1} w_{2}}{\sin (\alpha) \sin (\gamma)} \frac{\sin ^{2}(\alpha) \sin (\gamma)}{w_{1} w_{2} w_{2}^{2}}=\frac{-\sin (\alpha)}{1-\cos ^{2}(\alpha) \cos ^{2}(\gamma-\beta)} .
\end{aligned}
$$


Before we consider the partial derivative of $g_{1,2}$ with respect to $\beta$ we prove the following identity:

$$
\begin{aligned}
& \sin ^{2}(\alpha) \cos ^{2}(\alpha) \sin ^{2}(\gamma) \sin (\gamma-2 \beta)=\cos ^{2}(\alpha) \sin (\gamma-2 \beta) w_{1}^{2} w_{2}^{2} \\
& \quad+\sin ^{2}(\alpha) \cos (\gamma) \cos ^{2}(\alpha) \cos (\beta) \sin (\beta) w_{2}^{2} \\
& \quad-\cos ^{4}(\alpha) \sin ^{2}(\beta) \sin (\gamma-\beta) \cos (\beta) w_{2}^{2} \\
& \quad-\sin ^{2}(\alpha) \cos (\gamma) \cos ^{2}(\alpha) \cos (\gamma-\beta) \sin (\gamma-\beta) w_{1}^{2} \\
& \quad+\cos ^{4}(\alpha) \sin (\beta) \cos (\gamma-\beta) \sin ^{2}(\gamma-\beta) w_{1}^{2}
\end{aligned}
$$

Dividing both sides by $\cos ^{2}(\alpha)$ gives

$$
\begin{aligned}
& \sin ^{2}(\alpha) \sin ^{2}(\gamma) \sin (\gamma-2 \beta)=\sin (\gamma-2 \beta) w_{1}^{2} w_{2}^{2} \\
& \quad+\sin ^{2}(\alpha) \cos (\gamma) \cos (\beta) \sin (\beta) w_{2}^{2} \\
& \quad-\cos ^{2}(\alpha) \sin ^{2}(\beta) \sin (\gamma-\beta) \cos (\beta) w_{2}^{2} \\
& \quad-\sin ^{2}(\alpha) \cos (\gamma) \cos (\gamma-\beta) \sin (\gamma-\beta) w_{1}^{2} \\
& \quad+\cos ^{2}(\alpha) \sin (\beta) \cos (\gamma-\beta) \sin ^{2}(\gamma-\beta) w_{1}^{2} .
\end{aligned}
$$

Again we make some elementary transformations

$$
\begin{aligned}
& \sin (\gamma-2 \beta) w_{1}^{2} w_{2}^{2} \\
& +\sin ^{2}(\alpha) \cos (\gamma) \cos (\beta) \sin (\beta) w_{2}^{2} \\
& -\cos ^{2}(\alpha) \sin ^{2}(\beta) \sin (\gamma-\beta) \cos (\beta) w_{2}^{2} \\
& -\sin ^{2}(\alpha) \cos (\gamma) \cos (\gamma-\beta) \sin (\gamma-\beta) w_{1}^{2} \\
& +\cos ^{2}(\alpha) \sin (\beta) \cos (\gamma-\beta) \sin ^{2}(\gamma-\beta) w_{1}^{2} \\
& \sin (\gamma-2 \beta) w_{1}^{2} w_{2}^{2} \\
& +\sin ^{2}(\alpha) \cos (\gamma) \cos (\beta) \sin (\beta) \\
& -\sin ^{2}(\alpha) \cos (\gamma) \cos (\beta) \sin (\beta) \cos ^{2}(\alpha) \cos ^{2}(\gamma-\beta) \\
& -\cos ^{2}(\alpha) \sin ^{2}(\beta) \sin (\gamma-\beta) \cos (\beta) \\
& +\cos ^{2}(\alpha) \sin { }^{2}(\beta) \sin (\gamma-\beta) \cos (\beta) \cos ^{2}(\alpha) \cos ^{2}(\gamma-\beta) \\
& -\sin ^{2}(\alpha) \cos (\gamma) \cos (\gamma-\beta) \sin (\gamma-\beta) \\
& +\sin ^{2}(\alpha) \cos (\gamma) \cos (\gamma-\beta) \sin (\gamma-\beta) \cos ^{2}(\alpha) \cos ^{2}(\beta) \\
& +\cos ^{2}(\alpha) \sin (\beta) \cos (\gamma-\beta) \sin { }^{2}(\gamma-\beta) \\
& -\cos ^{4}(\alpha) \sin (\beta) \cos (\gamma-\beta) \sin { }^{2}(\gamma-\beta) \cos ^{2}(\beta) \\
& \sin ^{2}(\gamma-2 \beta) w_{1}^{2} w_{2}^{2} \\
& +\sin ^{2}(\alpha) \cos (\gamma)\left(\cos (\beta) \sin (\beta)-\cos (\gamma-\beta) \sin ^{2}(\gamma-\beta)\right) \\
& +\cos ^{2}(\alpha) \sin (\beta) \sin (\gamma-\beta) \times \\
& \left.+\cos ^{2}(\gamma-\beta) \sin (\gamma-\beta)-\sin (\beta) \cos (\beta)\right) \\
& +\cos ^{4}(\alpha) \sin (\beta) \sin (\gamma-\beta) \cos (\gamma-\beta) \cos ^{2}(\beta) \times \\
&
\end{aligned}
$$




$$
\begin{aligned}
& (\sin (\beta) \cos (\gamma-\beta)-\sin (\gamma-\beta) \cos (\beta)) \\
& +\sin ^{2}(\alpha) \cos ^{2}(\alpha) \cos (\gamma) \cos (\beta) \cos (\gamma-\beta) \times \\
& (\sin (\gamma-\beta) \cos (\beta)-\sin (\beta) \cos (\gamma-\beta)) \\
& \sin (y-x) \cos (y+x)= \\
& \sin (y) \cos (y)-\sin (x) \cos (x) \\
& \sin (\gamma-2 \beta) w_{1}^{2} w_{2}^{2} \\
& +\sin ^{2}(\alpha) \cos (\gamma)(-\cos (\gamma) \sin (\gamma-2 \beta)) \\
& +\cos ^{2}(\alpha) \sin (\beta) \sin (\gamma-\beta)(\cos (\gamma) \sin (\gamma-2 \beta)) \\
& +\cos ^{4}(\alpha) \sin (\beta) \sin (\gamma-\beta) \cos (\gamma-\beta) \cos (\beta) \times \\
& (\sin (\beta) \cos (\gamma-\beta)-\sin (\gamma-\beta) \cos (\beta)) \\
& +\sin ^{2}(\alpha) \cos ^{2}(\alpha) \cos (\gamma) \cos (\beta) \cos (\gamma-\beta) \times \\
& (\sin (\gamma-\beta) \cos (\beta)-\sin (\beta) \cos (\gamma-\beta)) \\
& \sin (x \pm y)=\sin (x) \cos (y) \\
& \pm \cos \left(\underline{=} \sin (y) \quad \sin (\gamma-2 \beta) w_{1}^{2} w_{2}^{2}\right. \\
& +\sin ^{2}(\alpha) \cos (\gamma)(-\sin (\gamma-2 \beta) \cos (\gamma)) \\
& +\cos ^{2}(\alpha) \sin (\beta) \sin (\gamma-\beta)(\cos (\gamma) \sin (\gamma-2 \beta)) \\
& +\cos ^{4}(\alpha) \sin (\beta) \sin (\gamma-\beta) \cos (\gamma-\beta) \cos (\beta) \times \\
& (-\sin (\gamma-2 \beta)) \\
& +\sin ^{2}(\alpha) \cos ^{2}(\alpha) \cos (\gamma) \cos (\beta) \cos (\gamma-\beta)(\sin (\gamma-2 \beta)) \\
& =\sin (\gamma-2 \beta) \times \\
& \left(w_{1}^{2} w_{2}^{2}-\sin ^{2}(\alpha) \cos ^{2}(\gamma)\right. \\
& +\cos ^{2}(\alpha) \sin (\beta) \sin (\gamma-\beta) \cos (\gamma) \\
& -\cos ^{4}(\alpha) \sin (\beta) \sin (\gamma-\beta) \cos (\gamma-\beta) \cos (\beta) \\
& \left.+\sin ^{2}(\alpha) \cos ^{2}(\alpha) \cos (\gamma) \cos (\beta) \cos (\gamma-\beta)\right) \\
& =\sin (\gamma-2 \beta) \times \\
& \left(1-\cos ^{2}(\alpha) \cos ^{2}(\beta)-\cos ^{2}(\alpha) \cos ^{2}(\gamma-\beta)\right. \\
& +\cos ^{4}(\alpha) \cos ^{2}(\beta) \cos ^{2}(\gamma-\beta) \\
& -\sin ^{2}(\alpha) \cos ^{2}(\gamma) \\
& +\cos ^{2}(\alpha) \sin (\beta) \sin (\gamma-\beta) \cos (\gamma) \\
& -\cos ^{4}(\alpha) \sin (\beta) \sin (\gamma-\beta) \cos (\gamma-\beta) \cos (\beta) \\
& \left.+\sin ^{2}(\alpha) \cos ^{2}(\alpha) \cos (\gamma) \cos (\beta) \cos (\gamma-\beta)\right) \\
& \cos ^{2}+\sin ^{2}=1 \quad \sin (\gamma-2 \beta) \times \\
& \left(\sin ^{2}(\alpha) \sin ^{2}(\gamma)\right.
\end{aligned}
$$




$$
\begin{aligned}
& +1-\cos ^{2}(\alpha) \cos ^{2}(\beta)-\cos ^{2}(\alpha) \cos ^{2}(\gamma-\beta) \\
& +\cos ^{4}(\alpha) \cos ^{2}(\beta) \cos ^{2}(\gamma-\beta)-\sin ^{2}(\alpha) \\
& +\cos ^{2}(\alpha) \sin (\beta) \sin (\gamma-\beta) \cos (\gamma) \\
& -\cos ^{4}(\alpha) \sin (\beta) \sin (\gamma-\beta) \cos (\gamma-\beta) \cos (\beta) \\
& \left.+\sin ^{2}(\alpha) \cos ^{2}(\alpha) \cos (\gamma) \cos (\beta) \cos (\gamma-\beta)\right) \\
& \cos ^{2}+\sin ^{2}=1 \quad \sin (\gamma-2 \beta) \times \\
& \left(\sin ^{2}(\alpha) \sin ^{2}(\gamma)\right. \\
& +\cos ^{2}(\alpha) \sin ^{2}(\beta)-\cos ^{2}(\alpha) \cos ^{2}(\gamma-\beta) \\
& +\cos ^{4}(\alpha) \cos ^{2}(\beta) \cos ^{2}(\gamma-\beta) \\
& +\cos ^{2}(\alpha) \sin (\beta) \sin (\gamma-\beta) \cos (\gamma) \\
& -\cos ^{4}(\alpha) \sin (\beta) \sin (\gamma-\beta) \cos (\gamma-\beta) \cos (\beta) \\
& \left.+\sin ^{2}(\alpha) \cos ^{2}(\alpha) \cos (\gamma) \cos (\beta) \cos (\gamma-\beta)\right) \\
& =\quad \sin (\gamma-2 \beta) \sin ^{2}(\alpha) \sin ^{2}(\gamma)+\sin (\gamma-2 \beta) \cos ^{2}(\alpha) \times \\
& \left(\sin ^{2}(\beta)-\cos ^{2}(\gamma-\beta)+\cos ^{2}(\alpha) \cos ^{2}(\beta) \cos ^{2}(\gamma-\beta)\right. \\
& +\sin (\beta) \sin (\gamma-\beta) \cos (\gamma) \\
& -\cos ^{2}(\alpha) \sin (\beta) \sin (\gamma-\beta) \cos (\gamma-\beta) \cos (\beta) \\
& \left.+\sin ^{2}(\alpha) \cos (\gamma) \cos (\beta) \cos (\gamma-\beta)\right) \\
& =\quad \sin (\gamma-2 \beta) \sin ^{2}(\alpha) \sin ^{2}(\gamma)+\sin (\gamma-2 \beta) \cos ^{2}(\alpha) \times \\
& \left(\sin ^{2}(\beta)-\cos ^{2}(\gamma-\beta)\right. \\
& +\cos ^{2}(\alpha) \cos (\beta) \cos (\gamma-\beta) \times \\
& (\cos (\beta) \cos (\gamma-\beta)-\sin (\beta) \sin (\gamma-\beta)) \\
& +\sin (\beta) \sin (\gamma-\beta) \cos (\gamma) \\
& \left.+\sin ^{2}(\alpha) \cos (\gamma) \cos (\beta) \cos (\gamma-\beta)\right) \\
& \cos (x \pm y)=\cos (x) \cos (y) \\
& \mp \sin (x) \sin (y) \\
& \sin (\gamma-2 \beta) \sin ^{2}(\alpha) \sin ^{2}(\gamma)+\sin (\gamma-2 \beta) \cos ^{2}(\alpha) \times \\
& \left(\sin ^{2}(\beta)-\cos ^{2}(\gamma-\beta)\right. \\
& +\cos ^{2}(\alpha) \cos (\beta) \cos (\gamma-\beta) \cos (\gamma) \\
& +\sin (\beta) \sin (\gamma-\beta) \cos (\gamma) \\
& \left.+\sin ^{2}(\alpha) \cos (\gamma) \cos (\beta) \cos (\gamma-\beta)\right) \\
& \cos ^{2} \stackrel{+\sin ^{2}=1}{=} \quad \sin (\gamma-2 \beta) \sin ^{2}(\alpha) \sin ^{2}(\gamma)+\sin (\gamma-2 \beta) \cos ^{2}(\alpha) \times \\
& \left(\sin ^{2}(\beta)-\cos ^{2}(\gamma-\beta)+\cos (\beta) \cos (\gamma-\beta) \cos (\gamma)\right. \\
& +\sin (\beta) \sin (\gamma-\beta) \cos (\gamma))
\end{aligned}
$$




$$
\begin{array}{ll}
\stackrel{\cos (x \pm y)=\cos (x) \cos (y)}{\mp \sin (x) \sin (y)}= & \sin (\gamma-2 \beta) \sin ^{2}(\alpha) \sin ^{2}(\gamma)+\sin (\gamma-2 \beta) \cos ^{2}(\alpha) \times \\
& \left(\sin ^{2}(\beta)-\sin ^{2}(\gamma) \sin ^{2}(\beta)-\cos (\beta) \cos (\gamma) \sin (\beta) \sin (\gamma)\right. \\
& +\sin (\beta) \sin (\gamma-\beta) \cos (\gamma)) \\
\cos ^{2}+\sin ^{2}=1 & \sin (\gamma-2 \beta) \sin ^{2}(\alpha) \sin ^{2}(\gamma)+\sin (\gamma-2 \beta) \cos ^{2}(\alpha) \times \\
& \left(\cos ^{2}(\gamma) \sin ^{2}(\beta)-\cos (\beta) \cos (\gamma) \sin (\beta) \sin (\gamma)\right. \\
& +\sin (\beta) \sin (\gamma-\beta) \cos (\gamma)) \\
& \\
\stackrel{\sin (x \pm y)=\sin (x) \cos (y)}{ \pm \cos (x) \sin (y)}= & \sin (\gamma-2 \beta) \sin ^{2}(\alpha) \sin ^{2}(\gamma)
\end{array}
$$

On account of (A.1.11) and (A.1.14) we obtain

$$
\begin{aligned}
\frac{\delta g_{1,2}}{\delta \beta}= & -\frac{w_{1} w_{2}}{\sin (\alpha) \sin (\gamma)} \times \\
& \left(\frac{\cos ^{2}(\alpha)(\cos (\beta) \sin (\gamma-\beta)-\sin (\beta) \cos (\gamma-\beta)) w_{1} w_{2}}{w_{1}^{2} w_{2}^{2}}-\right. \\
& {\left[\frac{\left(-\sin ^{2}(\alpha) \cos (\gamma)+\cos ^{2}(\alpha) \sin (\beta) \sin (\gamma-\beta)\right)}{w_{1}^{2} w_{2}^{2}}\{\right.} \\
& \left.\left.\left.\cos ^{2}(\alpha) \cos (\beta) \sin (\beta) \frac{w_{2}}{w_{1}}-\cos ^{2}(\alpha) \cos (\gamma-\beta) \sin (\gamma-\beta) \frac{w_{1}}{w_{2}}\right\}\right]\right) \\
= & -\frac{w_{1} w_{2}}{\sin ^{2}(\alpha) \sin (\gamma)} \times \\
& \left(\frac{\cos ^{2}(\alpha) \sin (\gamma-2 \beta) w_{1} w_{2}}{w_{1}^{2} w_{2}^{2}}-\right. \\
& {\left[\frac{\left(-\sin ^{2}(\alpha) \cos (\gamma)+\cos ^{2}(\alpha) \sin (\beta) \sin (\gamma-\beta)\right)}{w_{1}^{2} w_{2}^{2}}\{\right.} \\
& \left.\left.\left.\cos ^{2}(\alpha) \cos (\beta) \sin (\beta) \frac{w_{2}}{w_{1}}-\cos ^{2}(\alpha) \cos (\gamma-\beta) \sin (\gamma-\beta) \frac{w_{1}}{w_{2}}\right\}\right]\right) \\
= & -\frac{1}{\sin ^{2}(\alpha) \sin (\gamma) w_{1}^{2} w_{2}^{2}} \times \\
& \left(\cos ^{2}(\alpha) \sin (\gamma-2 \beta) w_{1}^{2} w_{2}^{2}-\right. \\
& +\sin ^{2}(\alpha) \cos (\gamma) \cos ^{2}(\alpha) \cos (\beta) \sin (\beta) w_{2}^{2} \\
& -\cos ^{4}(\alpha) \sin (\beta) \sin ^{2}(\gamma-\beta) \cos (\beta) w_{2}^{2} \\
& -\sin ^{2}(\alpha) \cos (\gamma) \cos ^{2}(\alpha) \cos (\gamma-\beta) \sin (\gamma-\beta) w_{1}^{2} \\
& \left.+\cos ^{4}(\alpha) \sin (\beta) \cos ^{2}(\gamma-\beta) \sin ^{2}(\gamma-\beta) w_{1}^{2}\right) \\
&
\end{aligned}
$$


A.1. Partial Derivatives of $f_{1,2}, f_{1,3}, f_{2,3}, g_{1,2}, g_{1,3}, g_{2,3}$

$$
=-\frac{\sin (\alpha) \cos ^{2}(\alpha) \sin (\gamma) \sin (\gamma-2 \beta)}{\left(1-\cos ^{2}(\alpha) \cos ^{2}(\beta)\right)\left(1-\cos ^{2}(\alpha) \cos ^{2}(\gamma-\beta)\right)} .
$$

Further from (A.1.11) follows

$$
\begin{aligned}
& \frac{\delta g_{1,2}}{\delta \alpha}=-\frac{w_{1} w_{2}}{\sin (\alpha) \sin (\gamma)} \times \\
& \quad\left(\frac{(-2 \sin (\alpha) \cos (\gamma) \cos (\alpha)-2 \cos (\alpha) \sin (\alpha) \sin (\beta) \sin (\gamma-\beta)) w_{1} w_{2}}{w_{1}^{2} w_{2}^{2}}-\right. \\
& \quad \frac{\left(-\sin ^{2}(\alpha) \cos (\gamma)+\cos ^{2}(\alpha) \sin (\beta) \sin (\gamma-\beta)\right)}{w_{1}^{2} w_{2}^{2}} \times \\
& \left.\quad\left[\frac{w_{2}}{w_{1}} \cos (\alpha) \sin (\alpha) \cos ^{2}(\beta)+\frac{w_{1}}{w_{2}} \cos (\alpha) \sin (\alpha) \cos ^{2}(\gamma-\beta)\right]\right)
\end{aligned}
$$

Hence

$$
\begin{aligned}
& \frac{\delta g_{1,2}}{\delta \alpha}(\alpha, \gamma / 2, \gamma)=\frac{-1}{\sin (\alpha) \sin (\gamma)} \times \\
& \left(\frac{\left(-2 \sin (\alpha) \cos (\alpha) \cos (\gamma)-2 \cos (\alpha) \sin (\alpha) \sin ^{2}(\gamma / 2)\right)}{1-\cos ^{2}(\alpha) \cos ^{2}(\gamma / 2)}\left(1-\cos ^{2}(\alpha) \cos ^{2}(\gamma / 2)\right)\right. \\
& \left.\frac{\left(-\sin ^{2}(\alpha) \cos (\gamma)+\cos ^{2}(\alpha) \sin ^{2}(\gamma / 2)\right)\left(2 \cos (\alpha) \sin (\alpha) \cos ^{2}(\gamma / 2)\right)}{1-\cos ^{2}(\alpha) \cos ^{2}(\gamma / 2)}\right) \\
& =\frac{-2 \cos (\alpha)}{\sin (\gamma)\left(1-\cos ^{2}(\alpha) \cos ^{2}(\gamma / 2)\right)} \times \\
& \left(\left(-\cos (\gamma)-\sin ^{2}(\gamma / 2)\right)\left(1-\cos ^{2}(\alpha) \cos ^{2}(\gamma / 2)\right)-\right. \\
& \left.\left(-\sin ^{2}(\alpha) \cos (\gamma)+\cos ^{2}(\alpha) \sin ^{2}(\gamma / 2)\right)\left(\cos ^{2}(\gamma / 2)\right)\right) \\
& =\frac{-2 \cos (\alpha)}{\sin (\gamma)\left(1-\cos ^{2}(\alpha) \cos ^{2}(\gamma / 2)\right)} \times \\
& \left(-\cos (\gamma)-\sin ^{2}(\gamma / 2)+\cos (\gamma) \cos ^{2}(\alpha) \cos ^{2}(\gamma / 2)\right. \\
& +\cos ^{2}(\alpha) \sin ^{2}(\gamma / 2) \cos ^{2}(\gamma / 2) \\
& \left.+\sin ^{2}(\alpha) \cos (\gamma) \cos ^{2}(\gamma / 2)-\cos ^{2}(\alpha) \sin ^{2}(\gamma / 2) \cos ^{2}(\gamma / 2)\right) \\
& \cos ^{2}+\sin ^{2}=1 \quad \frac{-2 \cos (\alpha)}{\sin (\gamma)\left(1-\cos ^{2}(\alpha) \cos ^{2}(\gamma / 2)\right)} \times \\
& \left(-\cos (\gamma)-\sin ^{2}(\gamma / 2)+\cos (\gamma) \cos ^{2}(\gamma / 2)\right)
\end{aligned}
$$




$$
\begin{array}{cl}
\stackrel{\substack{\cos (x)=\cos (x / 2)^{2} \\
-\sin (x / 2)^{2}}}{=} & \frac{-2 \cos (\alpha)}{\sin (\gamma)\left(1-\cos ^{2}(\alpha) \cos ^{2}(\gamma / 2)\right)} \cos ^{2}(\gamma / 2)(\cos (\gamma)-1) \\
\stackrel{\substack{1-\cos (x)=\\
\sin (x / 2)^{2}}}{=} & \frac{-2 \cos (\alpha)}{\sin (\gamma)\left(1-\cos ^{2}(\alpha) \cos ^{2}(\gamma / 2)\right)} \cos ^{2}(\gamma / 2)\left(-2 \sin ^{2}(\gamma / 2)\right) \\
\stackrel{\sin (x) \cos (x)}{=} & \frac{\cos (\alpha) \sin (\gamma)}{\left(1-\cos ^{2}(\alpha) \cos ^{2}(\gamma / 2)\right)} \\
\stackrel{! ? ! ? ?}{=} & \left(-\frac{1}{2} \frac{\sin (\gamma) \cos (\alpha)\left(\cos ^{2}(\alpha)(\cos (\gamma)+1)-2\right)}{\left(1-\cos ^{2}(\alpha) \cos ^{2}(\gamma / 2)\right)^{2}}\right)
\end{array}
$$

Remark A.1.3 Let

$$
g_{1,3}=\arccos \left(\frac{-\cos (\alpha) \sin (\gamma-\beta)}{\sqrt{1-\cos ^{2}(\alpha) \cos ^{2}(\gamma-\beta)}}\right) .
$$

Then

$$
\begin{aligned}
\frac{\delta g_{1,3}}{\delta \gamma} & =\frac{\sin (\alpha) \cos (\alpha) \cos (\gamma-\beta)}{1-\cos ^{2}(\alpha) \cos ^{2}(\gamma-\beta)} \\
\frac{\delta g_{1,3}}{\delta \beta} & =\frac{-\sin (\alpha) \cos (\alpha) \cos (\gamma-\beta)}{1-\cos ^{2}(\alpha) \cos ^{2}(\gamma-\beta)} \\
\frac{\delta g_{1,3}}{\delta \alpha}(\alpha, \gamma / 2, \gamma) & =\frac{-\sin (\gamma / 2)}{1-\cos ^{2}(\alpha) \cos ^{2}(\gamma / 2)} .
\end{aligned}
$$

Proof. Again let $w_{2}=\sqrt{1-\cos ^{2}(\alpha) \cos ^{2}(\gamma-\beta)}$.

$$
\begin{aligned}
\frac{\delta g_{1,3}}{\delta \gamma}= & \frac{-1}{\sqrt{1-\left(\frac{-\cos (\alpha) \sin (\gamma-\beta)}{w_{2}}\right)^{2}} \times} \\
& \left(\frac{-\cos (\alpha) \cos (\gamma-\beta) w_{2}}{w_{2}^{2}}-\frac{-\cos (\alpha)^{3} \sin ^{2}(\gamma-\beta) \cos (\gamma-\beta) \frac{1}{w_{2}}}{w_{2}^{2}}\right) \\
= & \frac{-w_{2}}{\sin (\alpha)} \times \\
= & \left.\frac{-\cos (\alpha) \cos (\gamma-\beta) w_{2}}{w_{2}^{2}}-\frac{-\cos (\alpha)^{3} \sin ^{2}(\gamma-\beta) \cos (\gamma-\beta) \frac{1}{w_{2}}}{w_{2}^{2}}\right) \\
& \sin (\alpha) w_{2}^{2} \times\left(-\cos (\alpha) \cos (\gamma-\beta)\left(1-\cos ^{2}(\alpha) \cos ^{2}(\gamma-\beta)\right)+\right. \\
& \left.\cos (\alpha)^{3} \sin ^{2}(\gamma-\beta) \cos (\gamma-\beta)\right)
\end{aligned}
$$




$$
\begin{aligned}
= & \frac{\cos (\alpha) \cos (\gamma-\beta)}{\sin (\alpha) w_{1}^{2}} \times \\
& \left(1-\cos ^{2}(\alpha) \cos ^{2}(\gamma-\beta)-\cos ^{2}(\alpha) \sin ^{2}(\gamma-\beta)\right) \\
\cos ^{2} \stackrel{\sin ^{2}=1}{=} & \frac{\cos (\alpha) \cos (\gamma-\beta)}{\sin (\alpha) w_{1}^{2}} \times\left(1-\cos ^{2}(\alpha)\right) \\
\cos ^{2}+\sin ^{2}=1 & \frac{\sin (\alpha) \cos (\alpha) \cos (\gamma-\beta)}{w_{1}^{2}}=\frac{\sin (\alpha) \cos (\alpha) \cos (\gamma-\beta)}{1-\cos ^{2}(\alpha) \cos ^{2}(\gamma-\beta)} .
\end{aligned}
$$

Replacing $\beta$ by $\gamma$ shows

$$
\frac{\delta g_{1,3}}{\delta \beta}=-\frac{\delta g_{1,3}}{\delta \gamma}=\frac{-\sin (\alpha) \cos (\alpha) \cos (\gamma-\beta)}{1-\cos ^{2}(\alpha) \cos ^{2}(\gamma-\beta)} .
$$

Finally, for the partial derivative with respect to $\alpha$ we get

$$
\begin{aligned}
\frac{\delta g_{1,3}}{\delta \alpha}= & \frac{-1}{\sqrt{1-\left(\frac{-\cos (\alpha) \sin (\gamma-\beta)}{w_{2}}\right)^{2}}} \times \\
& \left(\frac{\sin (\alpha) \sin (\gamma-\beta) w_{2}}{w_{2}^{2}}-\frac{-\cos ^{2}(\alpha) \cos ^{2}(\gamma-\beta) \sin (\gamma-\beta) \sin (\alpha) \frac{1}{w_{2}}}{w_{2}^{2}}\right) \\
= & \frac{-w_{2}}{\sin (\alpha)} \times \\
= & \frac{-\sin (\gamma-\beta)}{w_{2}^{2}} \times\left(1-\cos ^{2}(\alpha) \cos ^{2}(\gamma-\beta)+\cos ^{2}(\alpha) \cos ^{2}(\gamma-\beta)\right) \\
= & \frac{-\sin (\gamma-\beta)}{1-\cos ^{2}(\alpha) \cos ^{2}(\gamma-\beta)}
\end{aligned}
$$

Remark A.1.4 Let

$$
g_{2,3}=\arccos \left(\frac{-\cos (\alpha) \sin (\beta)}{\sqrt{1-\cos ^{2}(\alpha) \cos ^{2}(\beta)}}\right) .
$$

Then

$$
\begin{aligned}
\frac{\delta g_{2,3}}{\delta \gamma} & =0 \\
\frac{\delta g_{2,3}}{\delta \beta} & =\frac{\sin (\alpha) \cos (\alpha) \cos (\beta)}{1-\cos ^{2}(\alpha) \cos ^{2}(\beta)} \\
\frac{\delta g_{2,3}}{\delta \alpha}(\alpha, \gamma / 2, \gamma) & =\frac{-\sin (\gamma / 2)}{1-\cos ^{2}(\alpha) \cos ^{2}(\gamma / 2)} .
\end{aligned}
$$


Proof. Since $g_{2,3}(\alpha, \beta, \gamma)=g_{1,3}(\alpha, \beta, 2 \beta)$ the remark is an immediate consequence of remark A.1.3. 


\section{References}

[Bal92] K. Ball, A lower bound for the optimal density of lattice packings, Internat. Math. Res. Notes 10 (1992), 217-221.

[Ban90] W. Banaszczyk, On the lattice packing-covering ratio of finite dimensional normed spaces, Colloq. Math. 59 (1990), 31-33.

[Bet93] U. Betke, The strong sausage conjecture in $E^{3}$, manuscript (1993).

[BF34] T. Bonnesen and W. Fenchel, Theorie der konvexen Körper, Springer, Berlin, 1934.

[BG84] U. Betke and P. Gritzmann, Über L. Fejes Tóth's Wurstvermutung in kleinen Dimensionen, Acta Math. Hungar. 43 (1984), 299-307.

[BGW82] U. Betke, P. Gritzmann and J.M. Wills, Slices of L. Fejes Tóth's sausage conjecture, Mathematica 29 (1982), 194-201.

[BH92] K. Böröczky, Jr. and M. Henk, Radii and the sausage conjecture, Canad. Math. Bull., Vol. 38 (2), (1995), 156-166.

[BHW94a] U. Betke, M. Henk and J.M. Wills, Finite and infinite packings, J. Reine Angew. Math. 53 (1994), 165-191.

[BHW94b] U. Betke, M. Henk and J.M. Wills, Sausages are good packings, Discrete Comput. Geom. (1994), to appear.

[Bli29] H.F. Blichfeldt, The minimum value of quadratic forms and the closest packing of spheres, Math. Ann. 101 (1929), 605-608.

[Bör92] K. Böröczky, Jr., Intrinsic volumes of finite ball-packings, Ph.D. thesis, University of Calgary, 1992.

[Bör93] K. Böröczky, Jr., About four-ball packings, Mathematica 40 (1993), $226-232$.

[Bör94] K. Böröczky, Jr., Mean projections and finite packings of convex bodies, Monats. Math. (1994), to appear. 
[BRZ64] R.P. Bambah, C.A. Rogers and H. Zassenhaus, On coverings with convex domains, Acta Arith. 9 (1964), 191-207.

[But72] G.J. Butler, Simultaneous packing and covering in Euclidean space, Proc. London Math. Soc. 25(3) (1972), 721-735.

[CS93] J.H. Conway and N.J.A. Sloane, Sphere packings, lattices and groups, 2nd ed., Springer, New York, 1993.

[Dow44] C.H. Dowker, On mimimum circumscribed polygons, Bull. Amer. Math. Soc. 50 (1944), 120-122.

[EGH89] P. Erdös, P.M. Gruber and J. Hammer, Lattice points, Longman Scientific \& Technical, Harlow, Essex/Wiley, New York, 1989.

[Fej50] L. Fejes Tóth, Some packing and covering theorems, Acta Sci. Math. (Szeged) 12/A (1950), 62-67.

[Fej72] L. Fejes Tóth, Lagerungen in der Ebene auf der Kugel und im Raum, 2nd ed., Springer, Berlin-New York, 1972.

[Fej75] L. Fejes Tóth, Research problem 13, Period. Math. Hungar. 6 (1975), 197-199.

[Fej83] G. Fejes Tóth, New results in the theory of packing and covering, Convexity and its Applications (Basel) (P.M. Gruber and J.M. Wills, eds.), Birkhäuser, Basel, 1983, pp. 318-359.

[FK93] G. Fejes Tóth and W. Kuperberg, Packing and covering with convex sets, Handbook of convex geometry (Amsterdam) (P.M. Gruber and J.M. Wills, eds.), vol. B, North-Holland, Amsterdam, 1993.

[GL87] P.M. Gruber and C.G. Lekkerkerker, Geometry of numbers, 2nd ed., North-Holland, Amsterdam, 1987.

[Gri84] P. Gritzmann, Finite Packungen und Überdeckungen, UniversitätGH-Siegen, 1984, Habilitationsschrift.

[Gri85] P. Gritzmann, Ein Approximationssatz für konvexe Körper, Geom. Dedicata 19 (1985), 277-286.

[Gri86] P. Gritzmann, Finite packing of equal balls, J. London Math. Soc. 33 (1986), 543-553.

[Gro60] H. Groemer, Über die Einlagerung von Kreisen in einem konvexen Bereich, Math. Z. 73 (1960), 285-294. 
[Gro63] H. Groemer, Existenzsätze für Lagerungen im Euklidischen Raum, Math. Z. 81 (1963), 260-278.

[Gro85] H. Groemer, Coverings and packings by sequences of convex sets, Discrete Geometry and Convexity (J.E. Goodman, E. Lutwak, J. Malkevitch and R. Pollack, eds.), vol. 440, New York Acad. Sci., New York, 1985, pp. 262-278.

[Grü67] B. Grünbaum, Convex polytopes, Interscience Publishers, John Wiley \& Sons, London, 1967.

[Gru93] P.M. Gruber, Geometry of numbers, Handbook of convex geometry (Amsterdam) (P.M. Gruber and J.M. Wills, eds.), vol. B, NorthHolland, Amsterdam, 1993.

[GW92] P.M. Gandini and J.M. Wills, On finite sphere packings, Math. Pannon. 3 (1992), 19-29.

[GW93] P. Gritzmann and J.M. Wills, Finite packing and covering, Handbook of convex geometry (Amsterdam) (P.M. Gruber and J.M. Wills, eds.), vol. B, North-Holland, Amsterdam, 1993.

[GZ92] P.M. Gandini and A. Zucco, On the sausage catastrophe in 4-space, Mathematica 39 (1992), 274-278.

[Hal94] T.C. Hales, The status of the Kepler conjecture, Math. Intelligencer 16 (1994), no. 3, 47-58.

[Hen94] M. Henk, Lattice refinements, manuscript (1994).

[Hla44] E. Hlawka, Zur Geometrie der Zahlen, Math. Z. 49 (1944), 285-312.

[Hla49] E. Hlawka, Ausfüllung und Überdeckung konvexer Körper durch konvexe Körper, Monatsh. Math. 53 (1949), 81-131.

[Hsi93] W.-Y. Hsiang, On the sphere packing problem and the proof of Kepler's conjecture, Internat. J. Math. 4 (1993), no. 5, 739-831.

[Hsi94] W.-Y. Hsiang, A rejoinder to Hales' article entitled "The status of the Kepler conjecture", manuscript (1994).

[Joh48] F. John, Extremum problems with inequalities as subsidiary conditions, Courant Anniversary Volume (New York), Interscience, New York, 1948, pp. 187-204.

[KL78] G.A. Kabatjanskiı̌ and V.I. Levenštein, Bounds for packings on a sphere and in space, Problemy Peredachi Informatsii 14 (1978), 3-25 (Russian), [Problems Inform. Transmission 14, 1-17]. 
[KPW84] P. Kleinschmidt, U. Pachner and J.M. Wills, On L. Fejes Tóth's sausage conjecture, Israel J. Math. 47 (1984), 216-226.

[Lee67] J. Leech, Note on sphere packings, Canad. J. Math. 19 (1967), 251267.

[Lei79] K. Leichtweiß, Konvexe Mengen, Springer, Berlin, 1979.

[Min96] H. Minkowski, Geometrie der Zahlen, Teubner, Leipzig-Berlin, 1896.

[Min05] H. Minkowski, Dichteste gitterförmige Lagerungen kongruenter Körper, Nachr. Ges. Wiss. Göttingen (1905), 311-355, (Ges. Abh. II, $3-42)$.

[MS71] P. McMullen and G.C. Shephard, Convex polytopes and the upper bound conjecture, Cambridge University Press, Cambridge, 1971.

[Mud93] D.J. Muder, A new bound on the local density of sphere packings, Discrete Comp. Geom. 10 (1993), 351-375.

[Ole61] N. Oler, An inequality in the geometry of numbers, Acta Math. 105(A) (1961), 19-48.

[Ran47] R.A. Rankin, On the closest packing of spheres in $n$ dimensions, Ann. of Math. 48 (1947), 1062-1081.

[Rei34] K. Reinhardt, Über die dichteste gitterförmige Lagerung kongruenter Bereiche in der Ebene und eine besondere Art konvexer Kurven, Abh. Math. Sem. Hansischer Univ. 10 (1934), 216-230.

[Rog50] C.A. Rogers, A note on coverings and packings, J. London Math. Soc. 25 (1950), 327-331.

[Rog51] C.A. Rogers, The closest packing of convex two-dimensional domains, Acta Math. 86 (1951), 309-321.

[Rog60] C.A. Rogers, The closest packing of convex two-dimensional domains, Acta Math. 104 (1960), 305-306, Corrigendum.

[Rog64] C.A. Rogers, Packing and covering, Cambridge Univ. Press, Cambridge, 1964.

[RS57] C.A. Rogers and G.C. Shephard, The difference body of a convex body, Arch. Math. 8 (1957), 220-233.

[Sch50] L. Schläfli, Gesammelte mathematische Abhandlungen, vol. I, Birkhäuser, Basel, 1950. 
[Sch61] W.M. Schmidt, Zur Lagerung kongruenter Körper im Raum, Monatsh. Math. 65 (1961), 154-158.

[Sch63] W.M. Schmidt, On the Minkowski-Hlawka theorem, Illinois J. Math. 7 (1963), 18-23.

[Sch93] R. Schneider, Convex bodies: The Brunn-Minkowski theory, Cambridge University Press, Cambridge, 1993.

[Sid73] V.M. Sidel'nikov, On the densest packing of balls on the surface of an $n$-dimensional Euclidean sphere and the number of binary code vectors with a given code distance, Dokl. Akad. Nauk SSSR 213 (1973), 10291032 (Russian), [Soviet Math. Dokl. 14, 1851-1855].

[Sid74] V.M. Sidel'nikov, New estimates for the closest packing of spheres in n-dimensional Euclidean space, Math. Sb. 95 (1974), 148-158 (Russian), [Math. USSR Sb. 24, 147-157].

[SY93] J.R. Sangwine-Yager, Mixed volumes, Handbook of convex geometry (Amsterdam) (P.M. Gruber and J.M. Wills, eds.), vol. A, NorthHolland, Amsterdam, 1993.

[Weg86] G. Wegner, Über endliche Kreispackungen in der Ebene, Studia Sci. Math. Hungar. 21 (1986), 1-28.

[Wil85] J.M. Wills, On the density of finite packings, Acta Math. Hungar. 46 (1985), 205-210. 


\section{Notation Index}

In the order of first occurrence in the text.

\begin{tabular}{|c|c|c|}
\hline$E^{d}$ & 1 & $d$-dimensional Euclidean space \\
\hline $\mathbb{R}$ & 1 & set of real numbers \\
\hline $\mathcal{K}^{d}$ & 1 & set of $d$-dimensional convex bodies \\
\hline $\operatorname{int}(S)$ & 1 & interior of $S$ \\
\hline $\mathcal{P}(K)$ & 1 & set of packing sets of $K$ \\
\hline$V(S)$ & 1 & volume of $S$ with respect to its affine hull \\
\hline$W_{\lambda}^{d}$ & 2 & $d$-dimensional cube with edge length $2 \lambda$ and center 0 \\
\hline$\delta(K, C)$ & 2 & density of the packing set $C$ of $K$ \\
\hline$\delta(K)$ & 2 & density of a densest (infinite) packing of $K$ \\
\hline $\mathcal{P}^{*}(K)$ & 2 & set of all packing lattices of $K$ \\
\hline$\delta^{*}(K)$ & 3 & density of a densest lattice packing \\
\hline $\operatorname{det}(\Lambda)$ & 3 & determinant of a lattice $\Lambda$ \\
\hline $\mathcal{K}_{0}^{d}$ & 3 & family of all centrally symmetric convex bodies \\
\hline $\mathrm{D} K$ & 4 & difference body \\
\hline$\zeta(d)$ & 4 & zeta-function \\
\hline$B^{d}$ & 5 & $d$-dimensional unit ball \\
\hline $\operatorname{conv}(S), \operatorname{conv} S$ & 6 & convex hull of a set $S$ \\
\hline $\mathcal{P}(K, n)$ & 6 & $\begin{array}{l}\text { set of all packing sets (configurations) of cardinality } n \text { with } \\
\text { respect to } K\end{array}$ \\
\hline$\#(C)$ & 6 & cardinality of the set $C$ \\
\hline$\delta_{1}(K, C)$ & 6 & (classical) density of the finite packing set $C$ \\
\hline$\delta_{1}(K, n)$ & 6 & $\begin{array}{l}\text { (classical) density of a densest finite packing of } K \text { with car- } \\
\text { dinality } n \text {. }\end{array}$ \\
\hline$\kappa_{j}$ & 7 & volume of the $j$-dimensional unit ball \\
\hline$V(L, i, K, d-i)$ & & \\
\hline$V_{i}(L, K)$ & 7 & $i$-th mixed volume of $L$ and $K$ \\
\hline$F_{2 K}(T)$ & 8 & $\begin{array}{l}\text { Minkowski-perimeter of a plane set } T \text { with respect to the } \\
\text { gauge-function of the body } 2 K\end{array}$ \\
\hline$F(T)$ & 9 & perimeter of the plane set $T$ \\
\hline
\end{tabular}




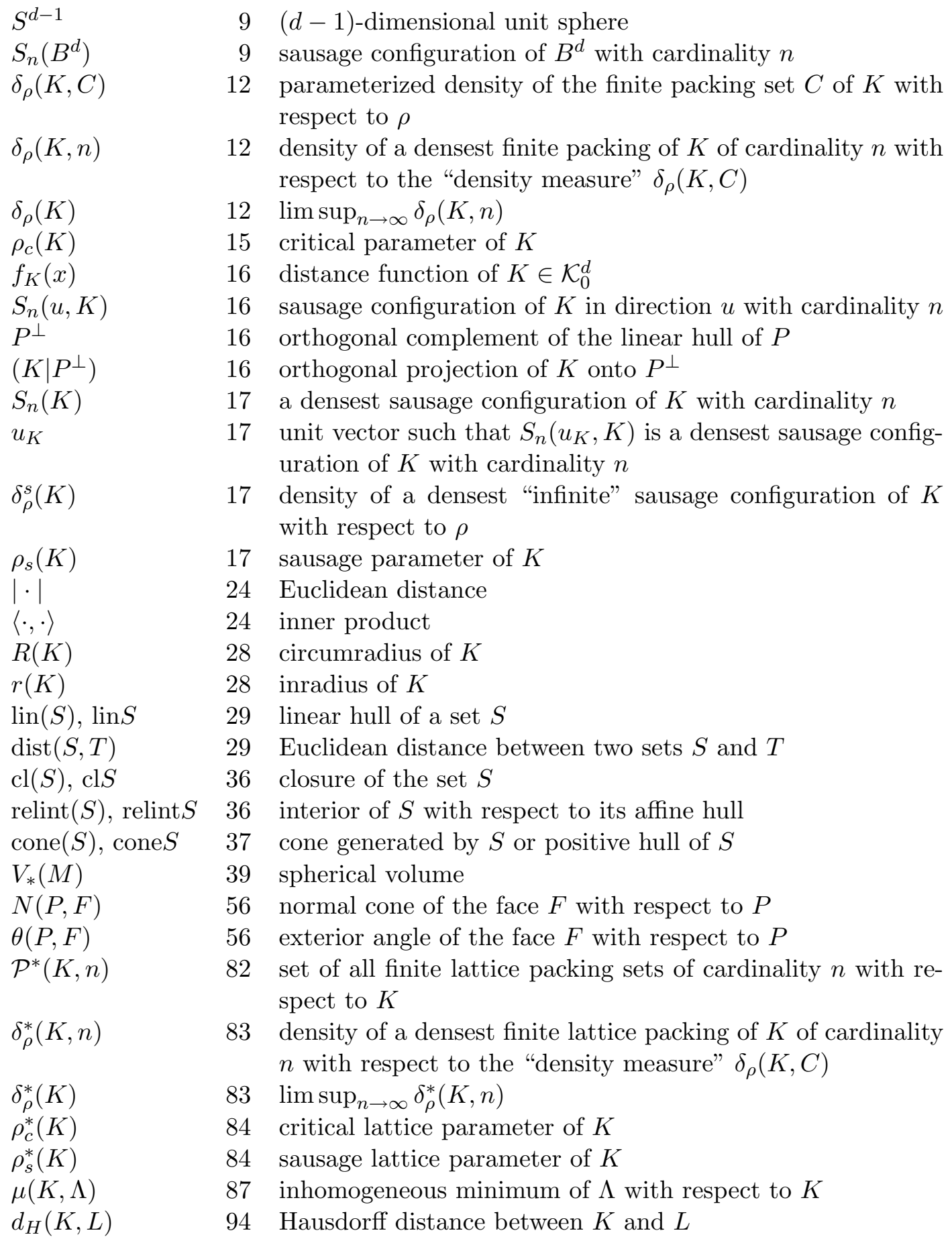

